\title{
Applications of systems biology to identify mechanisms of adaptive response to targeted single and combinatorial drug treatment in cancer
}

\author{
Brian James Capaldo \\ Ruckersville, Virginia
}

\begin{abstract}
A Dissertation presented to the Graduate Faculty of the University of Virginia in Candidacy for the Degree of

Doctor of Philosophy
\end{abstract}

Department of Biochemistry and Molecular Genetics

University of Virginia

December, 2016

Stefan Bekiranov, Ph.D.

Michael Weber, Ph.D.

Daniel Gioeli, Ph.D.

Charles Farber, Ph.D.

William Pearson, Ph.D. 
"There are three kinds of lies: lies, damned lies, and statistics." Attributed to Benjamin Disraeli, popularized by Mark Twain

This dissertation is dedicated to my wife, because she supported me through the struggles of being a graduate student, and my family, who have supported me through the Herculean amount of schooling I have chosen to undertake. 


\section{Acknowledgements}

I wish to first acknowledge both Aaron Mackey and Stefan Bekiranov. Aaron was magnanimous enough to teach me how to leverage computer programming as a biologist, while I had almost no experience with programming languages. He sat me down whenever was required and gave excellent guidance on how to track down the last little bug in the code. Additionally, he taught me the importance of including sanity checks in all my programs. At the bench top, it can often be half a day of experiments before you get to see results that can be interpreted. At the desktop, you have the option of observing your results at every step of the way, and Aaron made sure I exploited this at every opportunity. Lastly, Aaron taught me the basics of statistics, a field that I was even more unaware of than programming, and made sure I could adequately communicate its principles to my colleagues. After Aaron left the university, Stefan was gracious enough to continue my graduate education.

Stefan was fundamental in sharpening my critical thinking. Whereas Aaron taught me how to do things, as is the case with many mentors during the initial years of a graduate education, Stefan focused on teaching me to think about why to do things. Stefan was instrumental in turning the skills I learned under Aaron's tutelage as a means of at arriving at significant and novel scientific results. Through many hours of discussions and iterations of programs, Stefan has shown me the importance of carrying hypotheses to logical conclusions and developing a means to generate supporting information for the hypotheses. Stefan's guidance served to hone my skills as a research scientist. 
I would also like to acknowledge Dan Gioeli, Mike Weber, and their respective labs. Without the efforts of them and their labs, I would have no data upon which to perform my computational assays. Dan and Mike also made sure that I kept up with the relevant biological literature and helped me frame questions of biological importance, rather than focusing purely on the latest and greatest versions of software. Dan and Mike were also instrumental in developing my hypothesis testing, not only through investigation of my methods, but also providing lab support to test specific hypotheses. Without their contributions, the significance of my work would not have been properly assayed. Finally, Mike Weber was steadfast in providing stipend support for me, and extracting comparable support from the BMG Department and the Graduate Programs Office.

Lastly, I would like to thank the past and current members of my committee, namely Bill Pearson and Charles Farber. I would also like to thank the Department of Biochemistry and Molecular Genetics, the Center for Public Health Genomics, and Emily Farber for her work in the core facility. Without her, samples generated by the lab would never have been turned into numbers for me to crunch. 
1.1 Thesis Justification ........................................................................................10

1.2 Introduction to melanoma............................................................................11

1.2.1 Melanoma is driven by the aberrant activation of the MAPK pathway in the

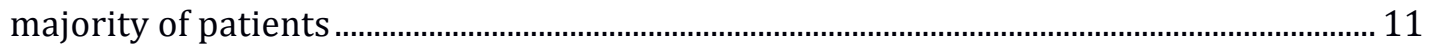

1.2.2 Activation of the MAPK pathway is accomplished through a cascade of dimerizations and phosphorylations

1.2.3 Vemurafenib is a potent inhibitor of mutant BRAF and is an exceptional therapeutic agent for melanoma.

1.2.4 Dimerization dependent mechanisms of resistance to vemurafenib treatment 15

1.2.5 Mechanisms of resistance to vemurafenib treatment independent of RAF dimerization...

1.2.6 Mechanisms of resistance to vemurafenib treatment independent of MAPK pathway reactivation...

1.2.7 Assessing sensitivity to vemurafenib......................................................................... 20

1.2.8 Combination therapies have been utilized as a means to overcome many resistance mechanisms observed in melanoma

1.3 Mantle cell lymphoma and chronic lymphocytic leukemia, two B-cell malignancies that benefit from combinatorial therapies.

1.3.1 Introduction to mantle cell lymphoma (MCL) and chronic lymphocytic leukemia (CLL) B-cell receptor (BCR) signaling pathway ……........................................... 28

1.3.2 Inhibition of BTK signaling is a potent therapeutic strategy in MCL and CLL ... 30

1.4 Integrative Analysis of Genomic and Functional Genomic Data Yield Insights into Mechanisms of Sensitivity and Resistance of Cancer Cells to Single and Combinatorial Drug Treatment.

1.4.1 Functional genomics is key to the effective implementation of personalized

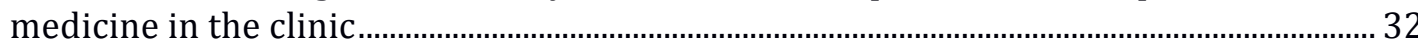
1.4.2 Unsupervised hierarchical clustering is a powerful tool for identifying common basal states and common responses to treatment

\section{Systems analysis of adaptive responses to MAP Kinase pathway blockade}

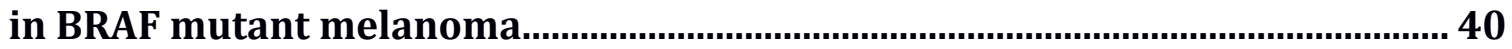

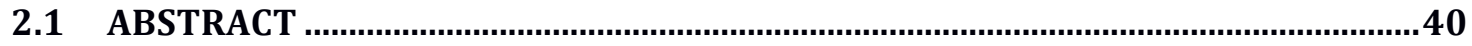

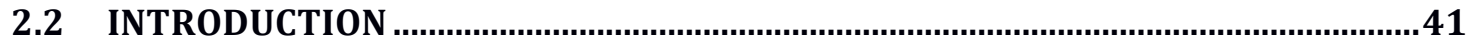

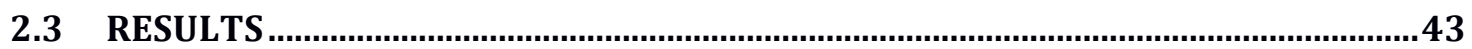

2.3.1 Analysis overview................................................................................................. 43

2.3.2 Analysis of the basal transcriptome yields groupings based on MITF and drug

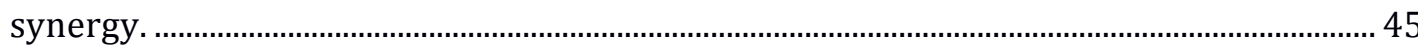

2.3.3 Grouping Cell Lines According to their Cytotoxicity Profiles is Consistent with

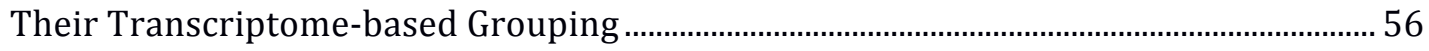

2.3.4 Exome Sequencing Reveals Mutations That Modulate Lineage-Based

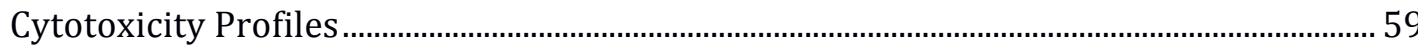

2.3.5 Cytotoxic Group-Wise Analysis After PLX4720 Treatment Reveals Broad ERBB

Pathway Gene Up-regulation in Combination Sensitive Cell Lines Only ………………..... 65

2.3.6 Reverse Phase Protein Array Analysis Reveals Down-regulation of Oncogenic

Proteins Across Cytotoxicity Groups Eight Hours After PLX4720 Treatment .................... 78

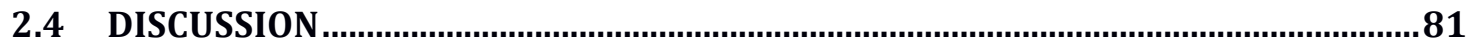

2.4.1 Analysis of the basal state: cytotoxicity, transcriptome and genome.................... 82

2.4.2 Adaptive responses to BRaf inhibition........................................................................... 86

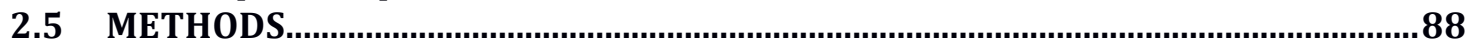


2.5.1 Cell Culture and Generation of Transcriptome, Proteome and Exome Data ...... 88

2.5.2 Statistical Analysis of Functional Genomics and Genomics Data........................... 90

2.6 ACKNOWLEGDEMENTS

3 Synergistic combination treatment of a subset of melanoma involves cotargeting of the MAPK pathway and PI3K/AKT signaling pathway via receptor tyrosine kinase inhibition .................................................................. 94

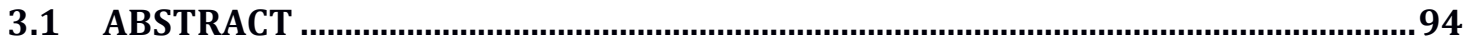

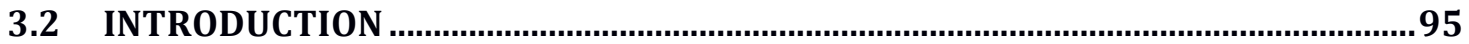

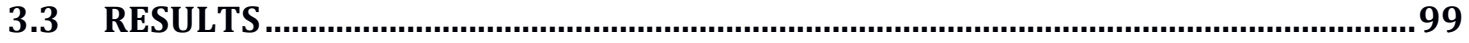

3.3.1 High throughput cytotoxicity screening of melanoma reveals synergistic combinations for treatment of cancer ................................................................................ 99

3.3.2 Basal signaling activity of the resistant lines when assayed by reverse phase protein arrays does not significantly differ from the lines sensitive to PLX4720 ........103

3.3.3 Cell lines show varying mechanisms of response after treatment with single drugs or the combination.

3.3.4 PLX4720 treatment, lapatinib treatment, or the combination treatment does not drive responses in DNA methylation .................................................................................116 3.3.5 Global hypomethylation induced by decitabine treatment sensitizes DM331 to lapatinib treatment

3.3.6 Decitabine treatment induces the same group of transcription factors that are induced by PLX4720 treatment in lines that respond synergistically to the combination of PLX4720 with lapatinib.

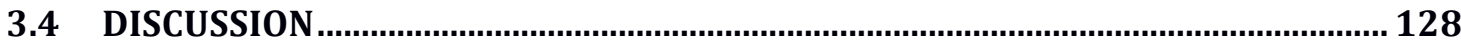

3.4.1 High throughput screening of combinations reveals pathway dependencies for melanoma 128

3.4.2 Targeting proximal pathways with drug combinations induces potentiative responses in the proteome and transcriptome

3.4.3 Combination of PLX4720 and lapatinib induces no appreciable changes in the methylome

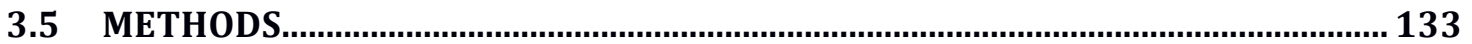

3.5.1 Cell culture and cytotoxicity data generation...........................................................133

3.5.2 Generation of transcriptome, proteome, DNA methylome and exome data.....134

3.5.3 Statistical Analysis of Functional Genomics Data .....................................................135

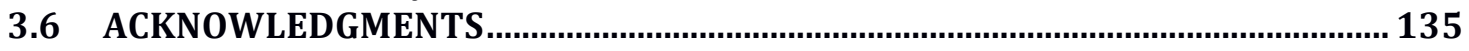

4 Combination therapy in melanoma and B cell malignancies demonstrates significant ability to overcome adaptive survival signaling responses ...........136

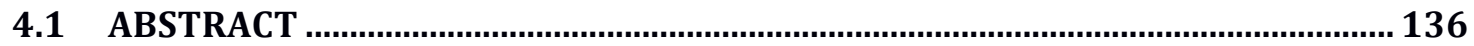

4.2 INTRODUCTION

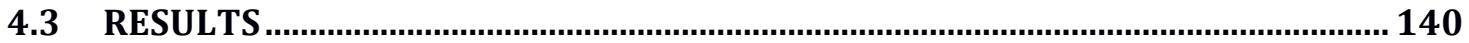

4.3.1 Sorafenib and diclofenac yield synergistic responses in melanoma regardless of

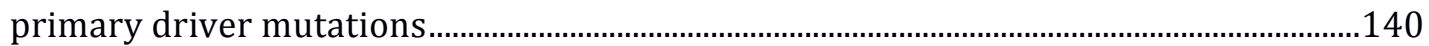
4.3.2 Synthetic lethal screening approach identifies secondary drugs that, when used in conjunction with ibrutinib, yield highly synergistic cytotoxic responses in MCL cell lines. 144

4.3.3 Transcriptomic responses to combination treatments reveal potential mechanisms of synergistic cytotoxic effects.

4.3.4 The combination of carfilzomib with ibrutinib appears to induce an extremely strong and robust apoptotic response via p53 signaling. 
4.3.5 The combination of ABT-199 and ibrutinib induces a transcriptional response

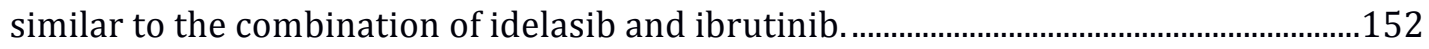

4.3.6 The apoptotic response to the combination of ABT-199 and ibrutinib is very swift, and is apparent when observed at the protein level.

4.3.7 Transcriptomic response to ibrutinib and ABT-199 combination reveals little insight into the mechanism of synergy.

4.3.8 Patient samples show significant responses to the combination of ABT199 and ibrutinib in vitro.

4.3.9 Patient sample transcriptomic response to the combination yields similar

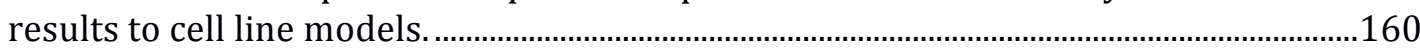

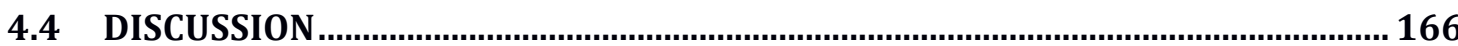

4.4.1 Distal combinations induce unique patterns of response in individual cell lines

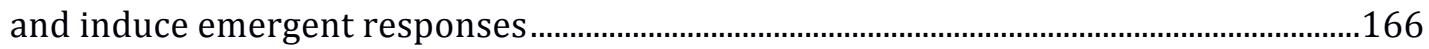

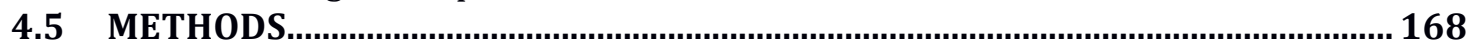

4.5.1 Cell culture and cytotoxicity data generation...........................................................168

4.5.2 Generation of transcriptome, proteome, DNA methylome and exome data.....170

4.5.3 Statistical Analysis of Functional Genomics..............................................................

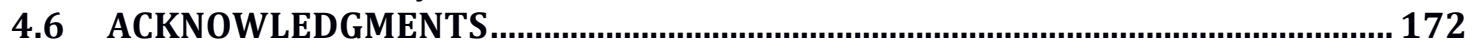

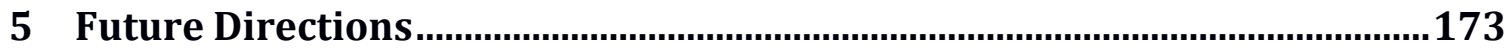

5.1 Combination therapy can overcome resistance to targeted monotherapy in

melanoma, chronic lymphocytic leukemia, and mantle cell lymphoma................... 173

5.2 The adaptive response to primary drugs reveals potential targets for secondary drug treatment.......................................................................................... 175

5.3 Integrative analysis revealed significant insights into the adaptive response

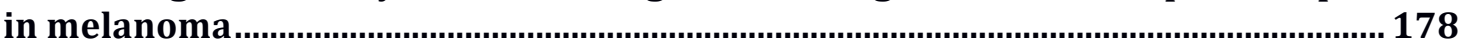

5.4 Emergent and potentiative combination responses appear dependent on

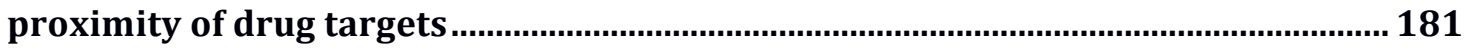

5.5 Combinations composed of distal drug pairs may perform better than

combinations composed of proximal drug pairs ........................................................... 182

5.6 Transcription factor enrichment analysis reveals a strong role for ETS family

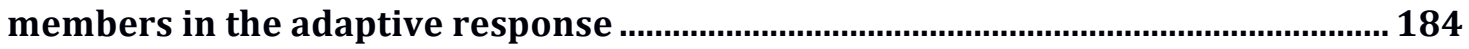

5.7 Observed adaptive responses to secondary drugs are blocked due to

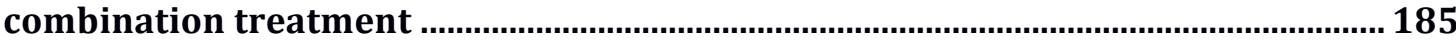

5.8 Leveraging phenocopying observed in previous screens could result in better

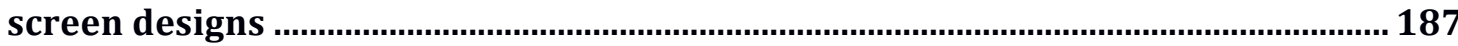

5.9 Computational integration of data could reveal additional specific

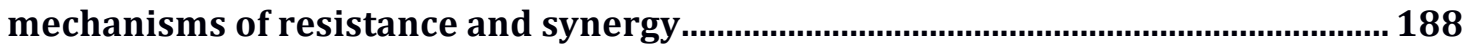

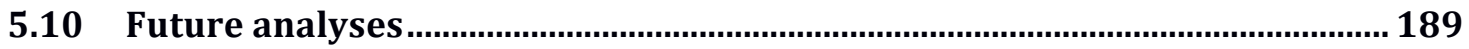

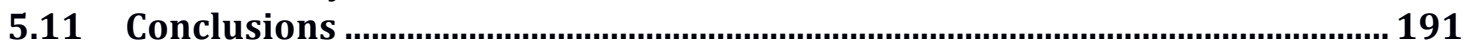

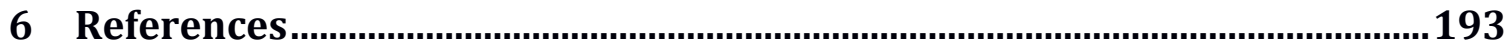


FIGURE 2.1 FUNCTIONAL GENOMIC DATA GENERATED AND ANALYSIS WORKFLOW.

FIGURE 2.2 CLUSTERING AND PCA ANALYSIS OF BASAL GENE EXPRESSION REVEALS MITF EXPRESSION AND GENE

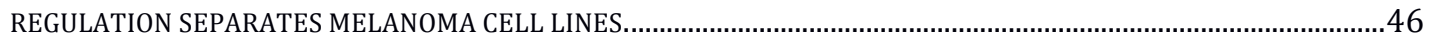

FIGURE 2.3 BLISS DIFFERENCE (SYNERGY SCORE) FOR LINES ACROSS 3 X 3 DOSE RESPONSE......................................50

FIGURE 2.4 OVERLAP OF CLUSTER IV GENES WITH GENES THAT CONTAIN MITF BINDING SITES AS IDENTIFIED BY CHIP SEQ

FIGURE 2.5 CYTOTOXICTY GROUPINGS ARE STRONGLY ASSOCIATED WITH PCA LOADINGS..........................................54

FIGURE 2.6 CLUSTERING BY CYTOTOXICITY REVEALS DIFFERENTIAL RESPONSES TO PLX4720 TREATMENT...............58

FIGURE 2.7 CYTOTOXIC RESPONSES TO SINGLE AND COMBINATION DRUG TREATMENTS FOR CELL LINES .....................59

FIGURE 2.8 EXOME ANALYSIS REVEALS TREND BETWEEN ZYGOSITY OF V600E ALLELE IN THE BRAF LOCUS AND

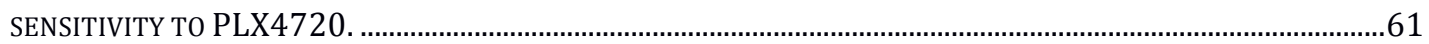

FIGURE 2.9 ERBB SIGNALING PATHWAY RESPONSE TO PLX4720 TREATMENT FOR EACH CYTOTOXICITY GROUP........69

FIGURE 2.10 PATHWAYS IN CANCER PATHWAY RESPONSE TO PLX4720 TREATMENT FOR EACH CYTOTOXICITY GROUP. .. .70

FIGURE 2.11 WNT SIGNALING PATHWAY RESPONSE TO PLX4720 TREATMENT FOR EACH CYTOTOXICITY GROUP.......71

FIGURE 2.12 MAPK SIGNALING PATHWAY RESPONSE TO PLX4720 TREATMENT FOR EACH CYTOTOXICITY GROUP. ...72

FIGURE 2.13 TRANSCRIPTION FACTORS ASSOCIATED WITH ERBB PATHWAY UPREGULATED IN COMBINATION SENSITIVE CELL LINES TREATED WITH PLX4720 ............................................................................................76

FIGURE 2.14 TRANSCRIPTION FACTOR ENRICHMENT OF ERBB SIGNALING PATHWAY FOR ALL LINES ...........................77

FIGURE 2.15 REVERSE PHASE PROTEIN ARRAY REVEALS STARK CHANGES TO OUTPUT OF RTK SIGNALING PATHWAYS

FIGURE 3.2 HEAT MAP OF ONE WAY ANOVA ANALYSIS OF BASAL PHOSPHO-EPITOPES DETERMINED BY RPPA....... 106

FIGURE 3.3 CHANGES IN PI3 KINASE AND MAP KINASE PATHWAY PROTEIN PHOSPHORYLATIONS IN RESPONSE TO DRUG TREATMENT

FIGURE 3.4 EACH LINE SHOWS UNIQUE PATTERN OF TRANSCRIPTIONAL RESPONSES TO SINGLE AND COMBINATION TREATMENT FOR PLX4720, MASITINIB, AND LAPATINIB

FIGURE 3.5 DNA METHYLATION STATUS OF DM331 DOES NOT SIGNIFICANTLY CHANGE IN RESPONSE TO SINGLE OR

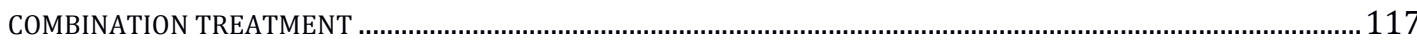

FIGURE 3.6 DM6 HAS A UNIQUE DNA METHYLATION PROFILE BASED ON ITS SEPARATION IN THE PRINCIPAL COMPONENT ANALYSIS

FIGURE 3.7 SEPARATION OF DM6 IN PCA IS ATTRIBUTED TO ITS GLOBALLY HYPOMETHYLATED STATE....................120

FigURE 3.8 GLOBAL KNOCKDOWN OF DNA METHYLATION SENSITIZES DM331 TO LAPATINIB TREATMENT ............ 122

FIGURE 3.9 GLOBAL KNOCKDOWN OF DNA METHYLATION USING DECITABINE IS MORE ROBUST IN DM331 THAN A375

FIGURE 3.10 DECITABINE TREATMENT IN DM331 INDUCES SIGNIFICANT ALTERATIONS TO ITS GENE EXPRESSION PROFILE

FIGURE 4.1 THE EFFECT OF DRUG COMBINATIONS ON GENOME-WIDE GENE EXPRESSION...

FIGURE 4.2 SCREENING WITH TARGETED SECONDARY AGENTS IDENTIFIES DRUG COMBINATIONS THAT SYNERGIZE WITH IBRUTINIB 146

FIGURE 4.3 SYNERGISTIC COMBINATIONS IN MCL LINES EXHIBIT STRONG TRANSCRIPTIONAL RESPONSES OF AN

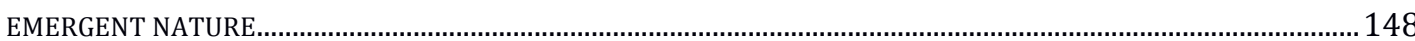

FIGURE 4.4 ABT199 TREATMENT APPEARS TO INDUCE APOPTOSIS VERY SWIFTLY .................................................154

FIGURE 4.5 TRANSCRIPTOMIC RESPONSES TO IBRUTINIB AND ABT AT TIME OF APOPTOSIS ......................................156

FIGURE 4.6 IBRUTINIB COMBINED WITH ABT-199 SHOWS STRONG RESPONSE IS EX VIVO MODELS OF PATIENT SAMPLES

FIGURE 4.7 EX VIVO RESPONSE TO COMBINATION IN PATIENT SAMPLES IS EXTREMELY PRONOUNCED. 162 
TABLE 2.1 MSIGDB TABLE FOR TRANSCRIPTION FACTOR ENRICHMENT FOR CLUSTERS III AND IV

TABLE 2.2 TOP 10 PATHWAYS FROM MSIGDB ENRICHMENT ANALYSIS FOR GENES THAT HAVE A NEGATIVE OR POSITIVE CORRELATION COEFFICIENT WITH THE SECOND PRINCIPAL COMPONENT ..............................................55

TABLE 2.3 PATHWAY EXPRESS ANALYSIS RESULTS FOR DIFFERENTIALLY EXPRESSED GENES IN RESPONSE TO PLX4720

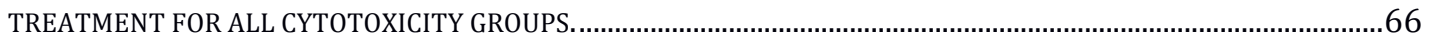

TABLE 2.4 MSIGDB TRANSCRIPTION FACTOR ENRICHMENT RESULTS FOR DIFFERENTIALLY EXPRESSED GENES IN

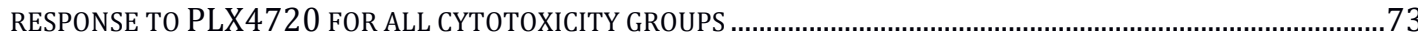

TABLE 3.1 DRUGS USED IN MELANOMA SCREEN, THE TARGETS, AND THE SUPPLIERS ...............................................100

TABLE 3.2 SECONDARY DRUGS THAT DEMONSTRATED SYNERGY IN AT LEAST ONE CELL LINE WHEN COMBINED WITH ONE OR MORE OF THE MAPK INHIBTORS.............................................................................................. 101

TABLE 3.3 MSIGDB PATHWAY ENRICHMENT OF DIFFERENTIALLY EXPRESSED GENES IN RESPONSE TO COMBINATION TREATMENT OF PLX4720 WITH EITHER LAPATINIB OR MASITINIB

TABLE 3.4 MSIGDB TRANSCRIPTION FACTOR ENRICHMENT RESULTS FOR GENES DIFFERENTIALLY EXPRESSED IN

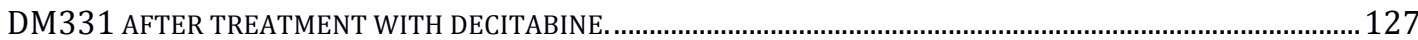

TABLE 4.1 SECONDARY DRUGS USED IN COMBINATION WITH IBRUTINIB................................................................. 144

TABLE 4.2 MSIGDB PATHWAY ENRICHMENT RESULTS FOR DIFFERENTIALLY EXPRESSED GENES IN RESPONSE TO TREATMENT WITH IBRUTINIB AND SECONDARY DRUGS IN JVM2 …............................................................ 150

TABLE 4.3 MSIGDB ENRICHMENT RESULTS FOR EX VIVO STUDY ON PATIENT SAMPLES EXPOSED TO IBRUTINIB AND ABT-199. 


\section{Introduction}

\subsection{Thesis Justification}

A central premise in modern cancer treatment is that better characterization of the genetics of each cancer should lead to better treatments and therefore better patient outcomes. Several improved high-throughput assays and functional genomics analyses currently exist to probe and characterize cancers by pinpointing specific genetic aberrations and their functional implications. But so far, few unambiguously positive outcomes have been observed.

Among all cancer types, melanoma has one of the highest mutational burdens. Although almost all melanomas are driven by mutated components of the mitogen activated protein kinase (MAPK) pathway, the genetic complexity and diversity of the disease creates multiple mechanisms to circumvent or overcome the effects of pathway blockade. Consequently, almost all melanoma patients relapse within 12 months of receiving treatment. Combination therapy has been shown to be effective in overcoming relapses, and some research indicates that combination therapy may be able to delay future relapses. However, effective combinations have thus far proved to be difficult to identify and no known combination of targeted agents provides more than a few months of additional life.

We employed a multipronged approach, combining functional genomics with high-throughput combination screening, to develop classification methods aimed not only at identifying effective therapeutic combinations, but also at determining potential biomarkers for responsiveness to these combinations, and at discerning 
mechanisms of synergy for some of these combinations. The tools we have developed for melanoma have also been applied to mantle cell lymphoma and chronic lymphocytic leukemia, to substantial effect.

\subsection{Introduction to melanoma}

\subsubsection{Melanoma is driven by the aberrant activation of the MAPK pathway in the majority of patients \\ Incidence of malignant melanoma is increasing among multiple}

demographics (Niezgoda et al., 2015), indicating investigation of therapies is becoming paramount. It is estimated that 132,000 new cases of melanoma will be diagnosed annually, and that the United States will see approximately 10,000 deaths from melanoma in the year 2015 (Niezgoda et al., 2015). In 2009, Stage IV metastatic melanoma patients had a median survival of 8 to 18 months (Balch et al., 2009) and modern therapies have only increased median survival times modestly with more recent trials showing overall survivals averaging 18.3 months (Niezgoda et al., 2015). At least $75 \%$ of cutaneous melanomas involve the activation of the MAPK pathway by genetic lesions (Niezgoda et al., 2015).

\subsubsection{Activation of the MAPK pathway is accomplished through a cascade of dimerizations and phosphorylations \\ The canonical MAPK pathway is crucial to the regulation of cell growth and}

division of many normal and cancer cells (Lito et al., 2012). Under normal growth

conditions, receptor tyrosine kinases, such as erb-b2 receptor tyrosine kinase (ERBB) family members, become activated by binding to extracellular ligands, such as neuregulin 1 (NRG1) and neuregulin 2 (NRG2), and lead to the phosphorylation of proteins that regulate rat sarcoma viral oncogene homolog (RAS) family 
members; neuroblastoma RAS viral (v-ras) oncogene homolog (NRAS), Kirsten rat sarcoma viral oncogene homolog (KRAS) or Harvey rat sarcoma viral oncogene homolog (HRAS), to adapt an active, guanosine triphosphate (GTP)-bound state (Downward, 2003). GTP-bound RAS family members can then induce the dimerization of the rapidly accelerating fibrosarcoma (RAF) family of kinases (Wellbrock et al., 2004), which is required for the activation of RAF family members (Farrar et al., 1996; Luo, 1996; Rushworth et al., 2006). The RAF kinase family consists of A-Raf proto-oncogene, serine/threonine kinase (ARAF), B-Raf protooncogene, serine/threonine kinase (BRAF), and Raf-1 proto-oncogene, serine/threonine kinase (RAF1 or CRAF) (Lito et al., 2013). The binding of RAS family members to RAF family members leads to translocation of the RAF family member to the membrane and the adoption of an "open" conformation (Marais et al., 1997). The RAF member can then be phosphorylated on residues residing in its $\mathrm{N}$ region, which is located near the C terminus (Fabian et al., 1993; Mason et al., 1999). These phosphorylations release the autoinhibitory nature of this domain, and are necessary for maximal kinase activity of RAF family members as well as for the binding of mitogen activated protein kinase kinase 1 (MAP2K1) and subsequent activation of MAP2K1 by phosphorylation on Serines 218 and 222 (Tran et al., 2005; Udell et al., 2011; Zhang and Guan, 2000). Activated RAF kinases target the protein kinases MAP2K1 and mitogen activated protein kinase kinase 2 (MAP2K2) for activation, which in turn activate the mitogen activated protein kinase 1 (MAPK1) and mitogen activated protein kinase 3 (MAPK3), leading to widespread activation of multiple targets that induce growth and cellular division (Lito et al., 2012). To 
date, MAPK1 has been shown to have over 50 different substrates (Lito et al., 2013) , including a host of negative feedback mechanisms that target regulators of RAS family members and MAPK1 itself (Lito et al., 2012, 2013). MAPK1 and MAPK3 activation lead to the negative regulation of the MAPK pathway via proteins that deactivate MAPK1, MAPK2, the ERBB family member epidermal growth factor receptor (EGFR), RAF family members, and RAS family members (Hanafusa et al., 2002; Lito et al., 2012). Members of the dual specificity phosphatases (DUSP) family and sprouty (SPRY) family are responsible for the inhibition of MAPK1 and MAPK3 (Lito et al., 2012).

$\mathrm{BRAF}^{\mathrm{V} 600 \mathrm{E}}$ is a mutated RAF kinase family member that appears frequently in melanoma. The incidence of this mutation varies from $30 \%$ to $72 \%$ in studies of melanoma patients (Niezgoda et al., 2015) and the constitutively active form of $\mathrm{BRAF}^{\mathrm{V} 600 \mathrm{E}}$ has been observed in multiple cancer types (Davies et al., 2002; Tiacci, 2011). The BRAF kinase is activated by the mutation from valine to glutamate at position 600 (BRAFV600E) (Curtin et al., 2005). The mutant BRAF activates the MAPK pathway (Sumimoto et al., 2004), signals as a monomer (Poulikakos et al., 2010), results in uncontrolled activation and expression of genes that are drivers of cell growth and migration (Joseph et al., 2010; Solit et al., 2006).

BRAF harbors a few key differences from other RAF members that explain the high frequency of BRAF mutations as drivers in cancer (Matallanas et al., 2011; Wellbrock et al., 2004). RAF1 has been the primary family member studied in determining the mechanism of RAF family member activation, and it has been shown that RAF family members contain a high degree of sequence similarity 
(Matallanas et al., 2011; Wellbrock et al., 2004). However, RAF1 is more stringently regulated than BRAF, as RAF1 requires multiple regulated phosphorylations to achieve full activity. BRAF is constitutively phosphorylated on a serine residue in the regulatory domain, and also contains a phosphomimetic aspartic acid within the $\mathrm{N}$ region at a site homologous to a RAF1 phosphorylation site (Mason et al., 1999). These variations allow BRAF to have a higher basal kinase activity than CRAF, which explains why a single mutation in BRAF can create a constitutively active oncogenic kinase (Wellbrock et al., 2004).

\subsubsection{Vemurafenib is a potent inhibitor of mutant BRAF and is an exceptional therapeutic agent for melanoma Vemurafenib is a targeted therapeutic compound designed against the} BRAF $^{\mathrm{V} 600 \mathrm{E}}$ kinase (Bollag et al., 2010; Dibb et al., 2004). It functions by binding near the dimerization site of the BRAF kinase proximal to the ATP binding cassette (Bollag et al., 2010). This binding event induces a shift in the protein's conformation at the $\mathrm{C}$ alpha helix, inhibiting the mutant form of BRAF kinase from phosphorylating its targets. Vemurafenib is extremely specific to its target (Joseph et al., 2010). This specificity was accomplished utilizing x-ray crystallographically assisted design (Tsai et al., 2008). The importance of BRAFV600E as an oncogenic driver is demonstrated by the rapid response of most $\mathrm{BRAFV}^{\mathrm{V} 00 \mathrm{E}}$ melanomas to the BRAF inhibitors vemurafenib or dabrafenib (Chapman et al., 2011; Hauschild et al., 2012). Over $50 \%$ of $B R A F^{V 600 E}$ melanomas showed objective response after treatment (Chapman et al., 2011; Hauschild et al., 2012). 
Unfortunately, response to vemurafenib is not durable; most patients relapse within 2 to 18 months of initial treatment (Flaherty et al., 2010, 2012a; Wagle et al., 2011). Several mechanisms of acquired resistance have been identified in melanoma. Mechanisms of resistance tend to involve the reactivation of the MAPK pathway (Lito et al., 2013). These mechanisms are either dependent on RAF dimerization (Johannessen et al., 2010; Montagut, 2008; Poulikakos et al., 2011; Rajakulendran et al., 2009; Shi et al., 2012) or independent of RAF dimerization (Antony et al., 2013; Wagle et al., 2011). There are also resistance mechanisms that do not depend on the reactivation of the MAPK pathway (Lito et al., 2013; Müller et al., 2014). When melanomas with BRAFV600E are treated with RAF inhibitors, such as vemurafenib, MAPK1 activity drops leading to a host of negative feedback mechanisms that act upstream in the pathway being released (Lito et al., 2012). This release from negative feedback allows resistant melanoma to adaptively respond in a matter of hours, and rapidly diminishes response to treatment (Lito et al., 2013).

\subsubsection{Dimerization dependent mechanisms of resistance to vemurafenib treatment \\ Among the changes induced by the release of negative feedback due to} vemurafenib treatment, higher concentrations of activated RAS family members contribute significantly to the adaptive response (Lito et al., 2013). As previously mentioned, activated RAS family members can stimulate the formation of dimers of RAF family members. Activation of RAF requires formation of homo- or heterodimers of RAF family members (Farrar et al., 1996; Luo, 1996; Rushworth et al., 2006). In addition to RAS contributing to the formation of dimers of RAF family members, vemurafenib will bind to wild type forms of the RAF family members, 
inducing RAF family members to dimerize. In the presence of activated RAS family members, BRAFV600E/BRAFWT and BRAFWT/RAF1 dimers can form with one monomer bound to vemurafenib, allowing for the other monomer, not bound to vemurafenib to be activated by activated RAS, leading to reactivation of the MAPK pathway (Poulikakos et al., 2010). Reactivation of the MAPK pathway is not limited to release of feedback inhibition by vemurafenib treatment, but genetic lesions and aberrant expression patterns have been identified that can also lead to the dimerization of RAF family members (Lito et al., 2013).

As previously mentioned, activated RAS family members are necessary to activate RAF family members in normal melanocytes (Downward, 2003). Typically, NRAS is the predominant RAS family member responsible for normal activation in cells of neural lineage, such as melanocytes (Lito et al., 2013). Mutation at position 61 puts NRAS into a constitutively active state, and NRASQ61 is the second most frequent driver of melanoma after BRAFV600E (Akbani et al., 2015). One of the first genetic adaptations to vemurafenib therapy observed in cell lines and patient samples were mutations in NRAS at position 61 (NRASQ61) (Nazarian et al., 2010). Under treatment with vemurafenib, resistant lines containing BRAFV600E and NRASQ61 utilize the constitutively active NRAS to overcome BRAFV600E inhibition. The mutant NRAS promotes dimerization of other RAF family members, independent of extracellular signals (Nazarian et al., 2010), resulting in reactivation of the MAP Kinase pathway independent of BRAFV600E inhibition.

Another means by which RAS can be activated in the cell to induce dimerization of RAF family members is via upregulation of Receptor Tyrosine 
Kinases (RTKs). Similarly to the NRASQ61 mutation, RTK activation, whether through aberrant overexpression of the receptor or its ligand, or constitutive activation due to a gain of function variant can drive melanoma in the absence of mutations in the MAPK pathway (Akbani et al., 2015). As described earlier, extracellular growth factors bind to RTKs and lead to the phosphorylation and activation of RAS (Downward, 2003). In a number of studies, RTKs and their associated ligands are observed to be upregulated in response to treatment with RAF inhibition (Lito et al., 2012; Nazarian et al., 2010; Villanueva et al., 2010). Many melanomas harbor biochemical aberrations in RTKs that can lead to their activation (Hartsough et al., 2014; Rebecca and Smalley, 2014). These can be any number of genetic (Basu et al., 2013; Prahallad et al., 2012) or epigenetic modulations (Halaban et al., 2009; Wang et al., 2014) that allow for activation of RTK signaling pathways. In these cases, nonmutated NRAS becomes activated as a consequence of RTK activation, other RAF family members become activated, and inhibition of BRAFV600E is no longer sufficient for inhibiting the MAP Kinase pathway and arresting tumor growth.

Accumulation of activated RAS, whether due to RTK activation or mutation to NRASQ61, is not the only means by which RAF family members can dimerize (Lito et al., 2013). In a study of five vemurafenib resistant tumors, one sample contained a BRAF splice variant that omitted exons 2-10. This omission was observed only in the RNA sequencing data, not in the exome, indicating that this protein variant was due to an alternative splicing event and not a genomic alteration (Wagle et al., 2014). This splicing event allows for RAS independent dimerization of BRAF, and this protein variant does not exclude the V600E substitution, which is present in exon 15 
(Greaves et al., 2013). Given the examples of resistant melanomas utilizing RAF dimerization as means of overcoming RAF inhibition, it can be hypothesized that any aberration that results in conditions that favor the dimerization of RAF family members could lead to resistance (Lito et al., 2013). Each of the mechanisms that drive dimerization of RAF family members also serve to reinforce the model that vemurafenib is effective at inhibiting monomeric mutant BRAF only (Lito et al., 2013).

\subsubsection{Mechanisms of resistance to vemurafenib treatment independent of} RAF dimerization

Reactivation of the MAPK pathway following vemurafenib treatment independent of dimerization of RAF family members can also be accomplished by RAF-independent reactivation of the MAP Kinase pathway. Two mechanisms of interest are mutated MAP2K1, and overexpression of mitogen-activated protein kinase kinase kinase 8 (MAPK3K8). MAP2K1 gain of function variants allow for the activation of MAPK pathway independent of phosphorylation by BRAF (Wagle et al., 2011). MAP3K8 is a kinase that will also activate MAP2K1, similarly to BRAF (Johannessen et al., 2010). In both cases, the MAPK pathway is becoming reactivated at the level of MAP2K1, below BRAF.

\subsubsection{Mechanisms of resistance to vemurafenib treatment independent of MAPK pathway reactivation \\ Resistance to BRAFV600E inhibition can involve activation of alternative} growth pathways that can diminish dependence on the MAPK pathway (Hartsough et al., 2014; Held et al., 2013; Kugel et al., 2014; Roller et al., 2012). Generally, $\mathrm{BRAF}^{\mathrm{V} 600 \mathrm{E}}$ melanomas strongly depend on signaling through the mutated BRAFV600E 
kinase (Curtin et al., 2005; Sumimoto et al., 2004) but there have been observations that melanomas circumvent dependence on $\mathrm{BRAF}^{\mathrm{V} 600 \mathrm{E}}$ signaling by utilizing v-akt murine thymoma viral oncogene homolog 1 (AKT1) signaling (Xing, 2012). Mutations in the phosphatidylinositol 3-kinases (PI3K) and AKT pathway are common in melanoma (Gray-Schopfer et al., 2007), and these pathways are capable of inducing growth like the MAPK pathway (Atefi et al., 2011). AKT signaling targets multiple downstream pathways, including PI3K-AKT-mammalian target of rapamycin (MTORC). Because the MAPK pathway and the PI3K/AKT signaling pathway are the primary signal transduction pathways for growth in melanoma, it is suspected that $\mathrm{BRAF}^{\mathrm{V} 600 \mathrm{E}}$ melanoma require mechanisms to activate PI3K/AKT signaling (Atefi et al., 2011; Goel et al., 2006). It has been shown that vemurafenib treatment can induce PI3K/AKT signaling in resistant lines (Atefi et al., 2011; Sánchez-Hernández et al., 2011). In addition to activation of the AKT/PI3K signaling pathway, other pathways are leveraged by melanomas to overcome vemurafenib treatment including signal transducer and activator of transcription (STAT) family members, the Hippo pathway effector YY1 associated protein 1 (YY1AP1), betacatenin, Bcl-2 homology domain 3 (BH3) proteins, autophagy and translational regulation (Atkinson et al., 2015; Corcoran et al., 2013; Davies, 2012; Delmas et al., 2015; Deuker et al., 2015; Gopal et al., 2014; Hartsough and Aplin, 2013; Hoeflich et al., 2012; Lassen et al., 2014; Lin et al., 2015; Ma et al., 2014; Pelletier et al., 2015; Rebecca et al., 2014; Schneider et al., 2014; Scortegagna et al., 2014, 2015; Shi et al., 2014a; Silva et al., 2014; Yadav et al., 2014). It is clear that given the various 
mechanisms of resistance, a means of determining sensitivity to treatment would be of clinical significance.

\subsubsection{Assessing sensitivity to vemurafenib}

The capability of vemurafenib to reactivate the MAPK pathway via

dimerization mechanisms (Halaban et al., 2010; Michaelis et al., 2014) led to investigations of tumor sensitivity to treatment with vemurafenib and the zygosity of the BRAFV60E allele (Søndergaard et al., 2010). While associations between sensitivity and copy number of the V600E allele are weak (Sapkota et al., 2013;

Søndergaard et al., 2010), the zygosity status of the mutant BRAF allele does modulate the adaptive response of the lines to treatment (Abel et al., 2013; Sapkota et al., 2013; Søndergaard et al., 2010). In a study of patient samples it was observed that BRAFV600 homozygotes tend to be more sensitive to vemurafenib treatment than BRAFV600E heterozygotes, and melanomas with BRAFWT tend to grow when in the presence of vemurafenib (Halaban et al., 2010). The trend of sensitivity was observed across a range of doses, but sensitivity did not correlate with basal levels of BRAF or RAF1 protein (Halaban et al., 2010). In a study of melanoma cell lines, a similar trend was observed between the zygosity status of the V600E allele at the BRAF locus and response to vemurafenib treatment (Søndergaard et al., 2010); however, in this study and the previous study, there were sensitive heterozygotes, all of which contained some level of deletions at the phosphatase and tensin homolog (PTEN) locus (Halaban et al., 2010; Søndergaard et al., 2010). When both studies examined the downstream effects, it was observed that vemurafenib treatment arrests growth in all lines, but induces apoptosis only in the sensitive 
lines (Halaban et al., 2010; Søndergaard et al., 2010). A BRAF amplification occurred in 4 out of 10 lines studied, but this amplification did not trend with sensitivity (Søndergaard et al., 2010). Given the weak associations between sensitivity and zygosity, later investigations focused on gene expression profiling to identify markers of sensitivity.

Microphthalmia-associated transcription factor (MITF) expression has been the most heavily investigated marker linked to vemurafenib sensitivity. MITF is strongly associated with the melanocyte lineage (Luciani et al., 2011; Osawa, 2009; Pshenichnaya et al., 2012), and is a master transcription factor responsible for inducing the transcription of melanocyte associated genes (Wellbrock et al., 2008). Melanoma is a cancer of melanocytes, cells in the skin that generate melanin, the pigment that provides protection from UV radiation and is responsible for hair and skin color (Denecker et al., 2014). Melanoblasts, derived from the neural crest, are the precursor cells for melanocytes and non-pigmented melanocyte stem cells (Ernfors, 2010). Interestingly, MITF overexpression has been shown to repress the ability of RAF inhibition to kill melanoma (Hertzman Johansson et al., 2013;

Johannessen et al., 2013) and serves as a marker of sensitivity to vemurafenib treatment (Konieczkowski et al., 2014; Müller et al., 2014).

Performing a near whole genome gain of function overexpression screen, it was discovered that overexpressed MITF led to marked increase in resistance to vemurafenib treatment (Johannessen et al., 2013). The observed resistance due to MITF overexpression could be overcome via the addition of inhibitors of histone deacetylases (HDAC), indicating that the resistance mechanism was converging on 
melanocyte lineage pathways to drive resistance to vemurafenib therapy (Johannessen et al., 2013). To ascertain the significance of the result, the researchers obtained patient samples to determine whether MITF expression levels were sensitive to MAPK inhibition. They observed that MITF levels decreased as the patients were treated with inhibitors of the MAPK pathway, and one patient that relapsed had a marked increase of MITF expression in their sample. Taken together, these results suggest that MITF overexpression is a mechanism of resistance to vemurafenib therapy. However, a follow up study showed that there is a strong correlation between MITF expression level (and its activity, as measured by expression levels of MITF target genes) and sensitivity to vemurafenib treatment. A whole genome screen of basal transcription levels of melanoma cell lines led to the observation that MITF expression is the strongest correlated gene with sensitivity to PLX4720 (Konieczkowski et al., 2014). MITF expression can be regulated by the activity of MAPK1 (Johannessen et al., 2013). Basal MAPK signaling activity did not account for the sensitivity to vemurafenib observed, and the correlation between MITF activity and sensitivity to MAPK inhibition holds true only for a limited number of drugs, including vemurafenib (Konieczkowski et al., 2014). Given the seemingly inconsistent nature of MITF expression and its poorly understood relationship with sensitivity to vemurafenib, more investigation is required.

A recent study that explored the relationship between MITF activity and vemurafenib sensitivity more stringently classified the expression levels of MITF within cell lines and the cell lines' responses to treatment. Again, the researchers observed that lines with high levels of MITF activity tend to be more sensitive to 
vemurafenib treatment (Müller et al., 2014). Cell lines treated with chronic doses of vemurafenib to generate resistant lines either maintained a high level of MITF activity, described as MITFacq_maint, or lost MITF activity completely, described as MITFacq_loss (Müller et al., 2014). Additionally, they report that the lines they worked with effectively segregated into two groups -- low expressing MITF lines, described as MITFendo_lo, and high expressing MITF lines, described as MITFendo_hi (Müller et al., 2014). They were able to determine that for lines that either acquire or lose MITF activity, some (MITFacq_maint lines) are resistant to BRAF inhibition only, whereas others (MITFacq_loss lines) are resistant to MAPK pathway inhibition via targeted therapy against multiple MAPK pathway members. Although this study served to increase the understanding of MITF and its role in sensitivity, it is unclear how clinically useful MITF would be as a marker of sensitivity.

\subsubsection{Combination therapies have been utilized as a means to overcome many resistance mechanisms observed in melanoma \\ Preventing the adaptive signaling response to RAF inhibition and}

overcoming the mechanisms of resistance described above has become of supreme importance in the clinical setting (Lito et al., 2013). Combination therapies can overcome both intrinsic or acquired resistance (Van Allen et al., 2014). Many of the mechanisms of intrinsic and acquired resistance are identical (Rebecca and Smalley, 2014), but acquired resistance mechanisms become manifested under the selective pressure introduced by treatment (Rebecca and Smalley, 2014). Depending on the mechanism of resistance, different combination strategies can be used. Compensatory signaling has been overcome by using a secondary drug that targets compensatory signaling pathways, such as targeting ERBB3 activity in vemurafenib 
treated melanomas (Kugel et al., 2014). Pathway reactivation can sometimes be overcome by targeting a downstream target of the primary drug, such as targeting MAP2K1 and BRAF in tandem (Flaherty et al., 2012a). In cases where a target mutation occurs, rendering the compound less effective, such target compensation can similarly be overcome by inhibiting downstream targets, such as targeting MAP2K1 in splice variant BRAF melanoma (Poulikakos et al., 2011). Identifying combinations is currently a resource intensive process.

There are systematic approaches for arriving at drug combinations; however, current approaches have serious limitations. Established practice is to screen and target oncogenic pathway genes/proteins that are upregulated in response to primary drug treatment (Abel et al., 2013). The problem with this approach is that expected MAPK and other known resistance pathways (PI3K, mTOR, apoptosis, cell cycle, etc.) (Martz et al., 2014) may not be the critical drivers of resistance, as we observed in our previous study in melanoma (Roller et al., 2012). Additionally, these candidate approaches tend to yield relatively little insight into the full response of the cell to primary treatment.

A complementary option for identifying combinations is drug screening based on a strategy that we (Roller et al., 2012) and other groups (Held et al., 2013) have used. Having found that a combination of sorafenib and diclofenac yields a synergistic cytotoxic response (Roller et al., 2012), we determined that the BRAF kinase and COX2, respectively, were being specifically targeted by these two drugs (Roller et al., 2012). With many available therapeutics to test, drug screening is a comprehensive and powerful approach for uncovering effective/synergistic 
combinations, but it is time consuming and expensive, and does not always yield mechanistic insights into resistance (Rebecca and Smalley, 2014). However, given that the drug targets are frequently known, screening does generate hypotheses that can be used to test mechanisms of resistance. Drug screening findings may also suggest functional genomic profiling experiments that can help uncover mechanisms of drug sensitivity, resistance, and synergy. We have observed that functional genomic screening of high-throughput drug treated data provides the most informative strategy for identifying drug combinations. We are able to identify broadly the pathways being affected by treatment, and determined those pathways as possible targets for secondary drugs in combinations through screening.

Screening for combinations, while time consuming and limited in its explanatory power, is still useful for arriving at targets. We initially screened compounds using a synthetic-lethality-based approach (Roller et al., 2012). To assess synergistic interactions, we used the Bliss model of independence (Zhao et al., 2014). We found that, in our melanoma screen, combinations that yielded greater than $26 \%$ synergy scores were in the top $98 \%$ for all combinations at all doses across our lines. In addition to the synergistic cytotoxicity of some combinations, there are a number of other advantages to combinations. Side effects might be reduced because drugs in combination are often effective at lower doses than when used separately (Smit et al., 2014). Lastly, combinations can overcome various forms of resistance, as previously described (Rebecca and Smalley, 2014; Smit et al., 2014). 
Two combinatorial strategies were initially proposed to overcome the adaptive signaling response observed in $\mathrm{BRAF}^{\mathrm{V} 600 \mathrm{E}}$ melanoma. The first strategy involved targeting MEK in addition to BRAF (Lito et al., 2012). The second strategy involved targeting RTKs in addition to BRAF (Straussman et al., 2012; Villanueva et al., 2010; Wilson et al., 2012). Preclinical models demonstrated efficacy of the first strategy (Lito et al., 2012), and clinical trials showed longer median time to progression for patients treated with the combination versus a monotherapy (Flaherty et al., 2012b). These findings were consistent with the paradigm that RAF inhibition causes an adaptive signaling response, via release of feedback inhibition of RAS and leading to reactivation of MAPK1. MAP2K1 inhibition combined with BRAF inhibition would thus be expected to ablate the MAPK pathway to an extent where MAPK reactivation is not possible (Lito et al., 2013). It has been observed that patients with tumors already resistant to vemurafenib show no response to this strategy (Falchook, 2012), and thus it must be employed in the setting of primary treatment. It has also been observed that this strategy reduces the occurrences of vemurafenib-dependent tumors, as well as the severity of vemurafenib-induced skin rashes (Lito et al., 2013), and thus represents one of those rare situations where the combination causes fewer side effects. Multiple clinical trials utilizing the strategy described above have been undertaken to significant success (Niezgoda et al., 2015). In January 2014, FDA approval of combined MAP2K1 and BRAF inhibition was announced. Additionally, this combination has repeatedly outperformed BRAF inhibitor monotherapy treatment of patients. However, this combination is not effective in patients who have demonstrated resistance to BRAF inhibitor 
monotherapy (Niezgoda et al., 2015). Regrettably, this strategy resulted in only a modest prolongation of survival (Niezgoda et al., 2015).

A second strategy has been proposed for overcoming the adaptive response to BRAFV600E inhibition, which involves targeting RTKs to reduce the increase of RAS activity that could activate other RAF family members, as described above (Lito et al., 2013). MAPK1 phosphorylation leads to the activation of negative regulators of RAS, and RAF inhibition leads to a decrease in these negative regulators. It has also been observed that RAF inhibition can lead to the activation of RTKs (Abel et al., 2013). In untreated BRAFV600 melanoma, RAS activity is kept at a minimum, but RTK activation can lead to its activation, and allow for RAS dependent RAF dimerization to reactivate the MAPK pathway (Lito et al., 2013). Additionally, RTKs can signal through multiple parallel growth pathways, leading to compensatory signaling around the MAPK pathway (Corcoran et al., 2010; Prahallad et al., 2012; Straussman et al., 2012; Villanueva et al., 2010; Wilson et al., 2012). A major obstacle preventing the adoption of this combination strategy is that RTK activation is extremely varied across cancer types, and even within melanoma samples (Lito et al., 2013).

To better overcome mechanisms of resistance in the clinical setting, studies have been undertaken to identify alternative combinations, combining BRAF inhibition with phosphatidylinositol-4,5-bisphosphate 3-kinase (PI3K), a component of the PI3K-AKT-mammalian target of rapamycin (mTOR) pathway (Atefi et al., 2011; Sullivan and Flaherty, 2013). However, the identification of novel combination therapies is of clinical significance. Using a screening based strategy, 
Held et. al determined (Held et al., 2013), that V600E BRAF mutant melanomas respond synergistically to a combination of PLX4720 and lapatinib-a result that we have confirmed (Roller et al., 2016). However, no hypothesis driven or genomic profiling experiments followed the drug screen; consequently, these studies yielded few insights into mechanisms of resistance or synergy.

In order (1) to determine mechanisms of resistance to PLX4720 and synergy of some lines to lapatinib and (2) to help arrive at systematic approaches to better predict which combinations might be effective/synergize, we followed these successful studies with a functional genomics and genetics profiling of 12 BRAFV600E melanoma cell lines. A multipronged genomics and functional genomics screening strategy allows for rapid iterative generation of hypotheses and testing of these hypotheses. As functional genomics data become cheaper and easier to produce, rapid iterative hypothesis testing becomes a key component to advancing the field.

\subsection{Mantle cell lymphoma and chronic lymphocytic leukemia, two B-cell malignancies that benefit from combinatorial therapies}

\subsubsection{Introduction to mantle cell lymphoma $(M C L)$ and chronic lymphocytic leukemia (CLL) B-cell receptor (BCR) signaling pathway MCL and CLL are B-cell malignancies that have poor prognoses; they are} dependent on the B-cell receptor (BCR) signaling pathway for growth (Byrd et al., 2013; Pérez-Galán et al., 2011; Swerdlow and Williams, 2002; Wang et al., 2013). The BCR signaling pathway begins with recognition of extracellular ligands by the BCR (Dalporto, 2004). The BCR is a complex composed of multiple proteins; it includes an antigen binding subunit, the membrane immunoglobulin (Ig), and a 
signaling subunit (which consists of a disulfide-linked heterodimer of Ig alpha and Ig beta) that associate in a non-covalent manner (Dalporto, 2004). A single immunoreceptor tyrosine-based activation motif (ITAM) is present on each of Ig alpha and Ig beta, and induces signal transduction following BCR aggregation (Flaswinkel and Reth, 1994). BCR aggregation induces ITAM phosphorylation, most likely by src-family kinases (Kurosaki, 1999). Following ITAM phosphorylation in both Ig alpha and Ig beta, the spleen tyrosine kinase (SYK) is recruited and activated by phosphorylation (Pao et al., 1998; Rowley et al., 1995). BCR expression at the cell surface is required for development and survival (Kitamura et al., 1991, 1992).

Proper BCR pathway signal transduction requires the ordered activation of three non-receptor protein tyrosine kinases. Traditionally, these are represented by LYN proto-oncogene, Src family tyrosine kinase (LYN), SYK, and Bruton agammaglobulinemia tyrosine kinase (BTK), which is required for BCR pathway signal transduction (Dalporto, 2004). LYN and SYK are recruited and activated by the BCR complex, following ligand recognition, and precede as well as influence BTK activation. BTK activation is also dependent on the generation of specific phospholipids by the action of PI3K (Gold et al., 2000; Scharenberg et al., 1998). The creation of these phospholipids allows for the recruitment of BTK (Saito et al., 2001; Scharenberg et al., 1998). Once LYN, SYK, and BTK have been activated, phospholipase C, gamma 2 (phosphatidylinositol-specific) (PLCG2) is recruited and activated, and an increase in intracellular $\left[\mathrm{Ca}^{2+}\right]$ is effected (Dalporto, 2004). PLCG2 recruitment and activation leads to the creation of second messengers that induce calcium ion mobilization. The calcium ion mobilization is required for activation of 
the nuclear factor kappa-light-chain-enhancer of activated B cells (NFkB) (Dolmetsch et al., 1997; Saijo et al., 2002; Trushin et al., 1999). One of the second messengers created by PLCG2 activity is diacyl-glycerol (DAG), which also activates protein kinase $\mathrm{C}(\mathrm{PKC})$ isotypes, which in turn regulate proteins associated with the MAPK pathway (Dalporto, 2004). The MAPK pathway is one of the primary integration points of BCR signaling, and consists of three members: MAPK1, mitogen-activated kinase 8 (MAPK8), and mitogen-activated kinase 11 (MAPK11) (Dong et al., 2002; Johnson and Lapadat, 2002). The BCR facilitates the activation of this pathway through the phospho-relay system described above. As seen in melanoma, the MAPK pathway can lead to survival and proliferation.

\subsubsection{Inhibition of BTK signaling is a potent therapeutic strategy in MCL and CLL}

Ibrutinib is a potent inhibitor of BTK, a kinase downstream of BCR. This drug has an exceptional overall response rate of $68 \%$ in patients with relapsed or refractory MCL, and 83\% in patients with relapsed or refractory CLL (Byrd et al., 2013; Wang et al., 2013). The highest response rates demonstrated by a single agent therapy in MCL or CLL (Axelrod et al., 2014a). However, as with melanoma, response to single agent therapy is not durable, and single agent therapy tends to induce adaptive responses that lead to drug resistance (Garraway and Jänne, 2012; Gioeli et al., 2011). Drug combinations that block adaptive signaling responses may prolong progression-free survival and overall survival in MCL (Liu et al., 2012; Pott et al., 2010). 
The development of a B-cell receptor antagonist has been a huge advance for the treatment of CLL (Woyach et al., 2014). As described above, BCR ligand interaction will induce proliferation, apoptosis, or anergy (Dalporto, 2004). In CLL, however, BCR ligand interactions induce proliferative and prosurvival signals (Bernal, 2001; Deglesne, 2006). BTK is not typically mutated in CLL (Puente et al., 2011; Wang et al., 2011); however, it is usually constitutively active (Herman et al., 2011; Honigberg et al., 2010). In vitro, ibrutinib irreversibly binds BTK, rendering the kinase inactive, inducing modest apoptosis in CLL and stopping BCR signaling (Herman et al., 2011; Ponader et al., 2012). Cases of relapse have been observed, but it is expected that the frequency of resistant tumors will only increase as the drug is prescribed to more patients (Woyach et al., 2014). Currently, two mechanisms of resistance have been observed. A mutation in BTK, a cysteine to serine change at position 481 BTK $^{\mathrm{C} 481 S}$, reduces the efficacy of the inhibitor by making the interaction between drug and target reversible and greatly reducing the affinity. The resistant mutations in PLCG2 allow for signal transduction from BCR independent of BTK, and have no effect on the BTK interaction with Ibrutinib (Woyach et al., 2014). This would suggest that, much like our melanoma studies, MCL and CLL possess many attributes that would make them prime candidates for further study. Much like melanoma, they have a well defined driver, with a targeted therapy available for the driver, as well as resistant tumors available for further study. 


\subsection{Integrative Analysis of Genomic and Functional Genomic Data Yield}

\section{Insights into Mechanisms of Sensitivity and Resistance of Cancer Cells to}

\section{Single and Combinatorial Drug Treatment}

\subsubsection{Functional genomics is key to the effective implementation of personalized medicine in the clinic}

Modern treatment of patients with cancer has been shown to be strongly dependent on our ability to classify their tumors as accurately as possible and prescribe appropriate treatments (Vaske et al., 2010). Many functional genomics analyses are currently available to ascertain the basal state of the cancer's genome, epigenome, transcriptome, and proteome. Additionally, we are able to ascertain the cancer's response to treatments in each of these assays and analyses, as well as evaluate the cytotoxic response to treatment of the cancer via high-throughput assays (Vaske et al., 2010). However, simple deductions from analyses of these highthroughput assays have yielded weak results. As described previously, $50 \%$ of all patients with $\mathrm{BRAF}^{\mathrm{V} 600 \mathrm{E}}$ melanoma who are treated with vemurafenib relapse with resistant disease within 12 months (Flaherty et al., 2010, 2012b; Wagle et al., 2011) and resistance to ibrutinib treatment in MCL and CLL patients is becoming more prevalent (Woyach et al., 2014). There has been limited success to effectively integrate modern technologies in order to develop better diagnostic and prognostic methods for patients (Vaske et al., 2010).

Genomic aberrations in cancer cells lead to the deregulation of growth pathways, and allow the genesis of the tumor (Hanahan and Weinberg, 2000, 2011). Genotype-phenotype associations are unclear because these genomic aberrations 
are often untested, novel, and many times unique to an individual cancer type, or even to an individual patient (Vaske et al., 2010). High-throughput functional genomics studies have made significant progress in better determining specific genotype-phenotype associations (Alizadeh et al., 2000; Golub et al., 1999; van de Vijver et al., 2002). These studies have been able to effectively establish gene expression profiles for specific cancer types, and The Cancer Genome Atlas (TCGA) project and others have furthered our understanding by highlighting the importance of using the gene lists generated from gene expression microarray studies and putting them in the context of pathway level analysis (Cancer Genome Atlas Research Network, 2008; Parsons et al., 2008). Viewing gene expression studies in the context of the pathways of which the genes were members allowed the researchers to determine that the same pathways usually were affected in patients, regardless of differences among patients in the aberrations found at the level of any individual gene. Additionally, these various aberrations tended to have the same effects on pathways, either activating or inhibiting a particular pathway (Cancer Genome Atlas Research Network, 2008; Parsons et al., 2008). These findings would indicate that even though cancers have varying genomic alterations, phenotypes observed at the pathway level can be similar. Developing effective pathway inhibition strategies may thus offer more promise than therapies targeted against specific genetic drivers of cancer.

Early pathway centric analyses have focused on correlating gene expression signatures with known phenotype or disease states and yielded promising results (Allison et al., 2006; Dudoit and Fridlyand, 2002; Tusher et al., 2001); they utilized 
analysis of variance and false discovery rates as parameters (Kerr et al., 2000; Storey and Tibshirani, 2003) to identify sets of genes to investigate further. To identify genes of significance in our studies, we used the R Bioconductor package Limma (Gentleman et al., 2004; Smyth, 2004) and its built-in tools to perform oneway ANOVA, T-tests, and paired T-tests. A one-way ANOVA calculates the variance for each gene across all the samples. These variances are then subjected to an F-test, which is used to determine whether there is at least one comparison between samples, for that gene, that is significant. Genes that pass the F-test at a significance threshold determined by false discovery rate correction are used as classifiers for that experiment (Kerr et al., 2000). T-tests are used to identify genes where comparisons between two samples (for example, a treatment and control for the same cell line) show a difference between the means of the two samples that is significantly different given the variance in the data of the samples (Smyth, 2004). A paired T-test is similar to a standard T-test, but it is used to identify differences that are of similar magnitude between paired samples (Smyth, 2004). As with the oneway ANOVA, significance was determined for these comparisons using a false discovery rate correction, which takes into account the number of comparisons being made (Storey and Tibshirani, 2003). Classifying these sets of genes into pathways was accomplished by utilizing overrepresentation-based statistical methods. Gene Set Enrichment Analysis (Subramanian et al., 2005) and Gene Ontology (Ashburner et al., 2000) were two of the first tools to utilize these statistics. Genes are ranked either by copy number alterations or by differential expression statistics, and pathways are ascribed significance based on how many 
genes from a given pathway are enriched in the top ranks of the list rather than the bottom. An offshoot of the GSEA is the Mutational Signatures Database, which uses a hypergeometric test on an unranked gene list. Genes of significance from the list are identified by some cutoff determined by the researcher, as described above. This list of genes is compared against the lists of genes for each pathway, and a p value is calculated to determine whether the submitted list of genes shows an enrichment for a pathway from the database (Subramanian et al., 2005). Overrepresentation analyses do not account for interdependencies among genes from the same pathway, and also assume that all genes equally contribute to affecting the pathways. Biological networks do not typically follow these assumptions. This can lead to overrepresentation analyses creating false negatives, by failing to identify pathways with several small changes across the whole pathway, and the creation of false positives, by identifying pathways that have only one gene or a few genes that have been selected, and have little to no result on the product of the pathway (Vaske et al., 2010).

Newer methods have been developed to better account for pathway topology in evaluating the significance of a pathway, given a list of genes (Efroni et al., 2007). Pathway topology can be obtained from a number of pathway databases, such as Reactome (Joshi-Tope et al., 2005), KEGG (Ogata et al., 1999), and the National Cancer Institute Pathway Interaction Database. One of the methods developed to take advantage of these databases is SPIA (Draghici et al., 2007). SPIA utilizes a method similar to Google's PageRank algorithm. A given gene's significance is dependent on the number of other genes it interacts with. When two genes have the 
same significance metric, the gene with more interactions would be ascribed a greater significance in its ability to perturb a pathway. The weakness of SPIA is that it only has the capability to interpret one data set, usually gene expression fold changes (Vaske et al., 2010). In our studies, we have obtained data on gene expression, protein phosphorylation, exome sequences, DNA methylation, copy number variations, and responses to treatment in many of these functional genomic studies, as well as cytotoxic response to these treatments. Analyzing data sets in isolation is insufficient to arrive at substantial hypotheses of mechanisms of synergy. It has been shown (and we have observed) that ERBB3 is upregulated in response to PLX4720 treatment and to Raf inhibition in general (Abel et al., 2013; Kugel et al., 2014; Lito et al., 2012). It was determined that PLX4720 treatment induces activation of FOXD3, and FOXD3 then mediates ERBB3 expression (Abel et al., 2013). Later, RPPA studies showed that PLX4720 treatment was also inducing NRG1, and NRG1 ligation with ERBB3 was stimulating AKT/PI3K signaling, a compensatory growth pathway that is parallel to the MAPK pathway (Kugel et al., 2014). We observe a similar pattern of ERBB3 and NRG1 upregulation in response to PLX4720 treatment, but we did not observe FOXD3 as a transcription factor driving the adaptive response to PLX4720 treatment. We suggest that the upregulation of the ErbB signaling pathway may be driven by ETS2, a known target of MAPK signaling (Oikawa and Yamada, 2003), and its cofactors in lines that are resistant to PLX4720. We were also able to identify this same pattern of transcription factors in another experiment, using decitabine, a global inhibitor of DNA methylation, which also led to the activation of the ErbB signaling pathway. 
Taken together, these results would indicate that lines resistant to PLX4720 treatment upregulate the ErbB signaling pathway through diverse transcription factors.

\subsubsection{Unsupervised hierarchical clustering is a powerful tool for identifying common basal states and common responses to treatment Unsupervised hierarchical clustering is a statistical method in which} similarity between samples within similar datasets can be investigated. We were able to utilize clustering to great effect on many of our datasets. Similarity between samples was calculated using the correlation distance metric. Correlation distance is defined as ( 1 - the correlation between two samples), and it is used to find trends between samples that are similar (Shimodaira, 2002; Suzuki and Shimodaira, 2006). Identifying trends, rather than absolute magnitude, is extremely important because genes that tend to be co-regulated display similar expression profiles across treatment and cell types, function in similar or related pathways and do not tend to have the same absolute level of expression.

In our studies, we have taken a synthetic lethal approach to identify drug combinations that cause synergistic cytotoxicity in a number of cancer types, utilizing the Bliss score of independence as a measure of synergy (Axelrod et al., 2014a, 2014c, 2014b; Roller et al., 2012). In our melanoma screen of 12 BRAFV600E mutants, we identified the combination of the vemurafenib analog PLX4720 in combination with lapatinib as being a potent therapy that induces synergistic cytotoxicity in six of the twelve lines assayed. Of note, the six lines that responded synergistically were also the most resistant to treatment with PLX4720 
monotherapy, as determined by IC50 assessment. The lines that did not show synergy were observed to be sensitive to monotherapy alone, potentially indicating that, in a patient setting, these melanomas would not require the combination initially and that any resistant recurrences would be sensitive to the combination. When we applied unsupervised hierarchical clustering to the results of the synthetic lethal drug screen, we observed that therapies that inhibited similar targets would also perform well in similar combinations, or that some combinations were synergistic in the same groups of lines, when the two combinations contained drugs that inhibited similar targets (Roller et al., 2016). We observed that melanomas tended to respond to the combination of BRAF and RTK inhibition, as noted, as a potential combination therapy (Lito et al., 2013).

We were also able to determine, using unsupervised hierarchical clustering, that cell lines that responded similarly to individual drugs and combinations have similar underlying biochemical responses to the individual drugs and combinations. For example, we identified a group of $\mathrm{BRAF}^{\mathrm{V} 600 \mathrm{E}}$ melanomas that clustered together in an assay of their cytotoxic responses to single and combination therapy. This group of melanomas showed a synergistic response to the combination, and we observed that they showed a broad activation of the ERBB/HER family signaling pathway, under treatment with PLX4720, that was unobserved in the rest of our cell lines. These results corroborated other studies of melanomas (Abel et al., 2013; Kugel et al., 2014), ; however we observed these responses at much lower dosages of the drugs than had been reported in studies of monotherapy. The proliferation/survival of cells with this compensatory signaling that we observed in 
this group of lines was effectively blocked in the presence of the combination treatment, which represents a promising therapy for future clinical use.

Utilizing unsupervised hierarchical clustering, we were also able to use the basal expression states of the lines to associate each cytotoxicity group with an expression profile dependent on MITF activity. Contrary to other studies, we observed that high MITF expressing lines show greater resistance to PLX4720 monotherapy (Konieczkowski et al., 2014), however we do not observe as large of a dynamic range of MITF expression that was observed previously. Our panel of lines is smaller and we use a different assay to determine cell viability. We have not yet been able to determine the mechanism by which MITF activity influences response to combination therapy, but, given the hypothesis that lineage influences RTK activity (Lito et al., 2013), we have been able to identify potential transcription factors that may be part of the response, as described above. We propose that interactions of BRAF zygosity and MITF transcriptional targets contribute to determining a melanoma's sensitivity to PLX4720 treatment and responsiveness to the combination of RAF and RTK inhibition. 


\section{Systems analysis of adaptive responses to MAP Kinase}

\section{pathway blockade in BRAF mutant melanoma.}

\subsection{ABSTRACT}

Fifty percent of cutaneous melanomas are driven by activated BRAFV600E, but tumors treated with RAF inhibitors, even when they respond dramatically, rapidly adapt and develop resistance. Thus, there is a pressing need to identify the major mechanisms of intrinsic and adaptive resistance and develop drug combinations that target these resistance mechanisms. In a combinatorial drug screen on a panel of 12 treatment-naïve BRAF ${ }^{\mathrm{V} 600 \mathrm{E}}$ mutant melanoma cell lines of varying levels of resistance to MAPK pathway inhibition we identified the combination PLX4720, a targeted inhibitor of mutated BRaf, and lapatinib, an inhibitor of the ERBB family of receptor tyrosine kinases, as synergistically cytotoxic in the subset of cell lines that displayed the most resistance to PLX4720. To identify potential mechanisms of resistance to PLX4720 treatment and synergy with lapatinib treatment we performed a multi-platform functional genomics analysis to profile the genome as well as the transcriptional and proteomic responses of these cell lines to treatment with PLX4720. We found modest levels of resistance correlated with the zygosity of the BRAF V600E allele and RTK mutational status. Layered over base-line resistance was substantial upregulation of many ERBB pathway genes in response to BRaf inhibition, thus generating the vulnerability to combination with lapatinib. The transcriptional responses of ERBB pathway genes are associated with a number of 
transcription factors, including ETS2 and its associated cofactors that represent a convergent regulatory mechanism conferring synergistic drug susceptibility in the context of diverse mutational landscapes.

\subsection{INTRODUCTION}

The BRaf kinase is activated by mutation from Valine to Glutamate at position 600 (V600E) in approximately 50\% of cutaneous melanomas (Curtin et al., 2005), resulting in uncontrolled activation of the MAP Kinase pathway and expression of genes that are drivers of cell growth and migration (Joseph et al., 2010; Solit et al., 2006). The importance of $\mathrm{BRAF}^{\mathrm{V} 600 \mathrm{E}}$ as an oncogenic driver is demonstrated by the rapid response of most BRAFV600E mutant melanomas to the BRaf inhibitors vemurafenib or dabrafenib (Chapman et al., 2011; Hauschild et al., 2012): Over 50\% of BRAF ${ }^{\mathrm{V} 600 \mathrm{E}}$ mutant melanomas showed objective response after treatment (Chapman et al., 2011; Hauschild et al., 2012).

Unfortunately, response to vemurafenib is not durable, with most patients relapsing within 2 to 18 months of initial treatment (Flaherty et al., 2010, 2012a; Wagle et al., 2011). Several mechanisms of acquired resistance have been identified in melanoma that has relapsed after treatment. For example, exogenous MITF overexpression has been shown to repress the ability of BRaf inhibition to kill melanoma (Hertzman Johansson et al., 2013; Johannessen et al., 2013) and in cells that display high MITF activity class III and class IV melanosomes can sequester drugs (Halaban et al., 2009). In more recent studies, lack of MITF activity has been implicated as an indication of resistance to BRaf inhibition (Konieczkowski et al., 
2014; Müller et al., 2014). Finally, a host of genomic alterations have been identified that circumvent the targeted inhibition of BRaf, in most cases reactivating the MAP Kinase pathway: BRAF splice variants facilitate dimerization with CRAF and result in MEK activation (Poulikakos et al., 2011); MEK can be activated by mutation or by activation of COT (Johannessen et al., 2010); the cytotoxic effects of MAPK pathway inhibition can be blunted by compensatory pathway activation, such as PI3K activation (Van Allen et al., 2014); and the zygosity of the V600E mutation is associated with modulating response to treatment with vemurafenib (Abel et al., 2013; Sapkota et al., 2013; Søndergaard et al., 2010). Less is known about mechanisms of intrinsic or adaptive resistance that can be manifested within a few hours or days of treatment, and is the focus of the current investigation.

Combination therapies are predicted to overcome intrinsic, adaptive and acquired resistance (Van Allen et al., 2014). For resistance acquired following relapse, DNA sequencing has uncovered mutational changes underlying resistance, and created the possibility for targeted combination therapies. However, there has been no systematic methodology established to predict effective combinations for newly diagnosed disease because of the complexity of the genetic changes in melanoma (Lawrence et al., 2014; Van Allen et al., 2014) and the consequent diversity of compensatory survival adaptations. Therefore, we and others (Held et al., 2013) have taken an empirical approach, performing high-throughput combinatorial screens of drugs and tool compounds to identify the most effective combinations of drugs or pathways for more durable melanoma treatment. We screened a panel of $12 \mathrm{BRAF}^{\mathrm{V} 600 \mathrm{E}}$ mutant melanoma cell lines, with a wide range of 
intrinsic sensitivity to the BRaf inhibitor PLX4720, in combination with a library of 60 secondary drugs and tool compounds. We found that the 6 lines most resistant to the BRaf inhibitor underwent synergistic cytotoxicity with lapatinib, an inhibitor of ERBB family receptor tyrosine kinases. In order to determine mechanisms of resistance to PLX4720 and synergy to lapatinib as well as help develop systematic approaches to better predict which combinations might be effective/synergize, we performed a functional genomics and genetics profiling of the 12 melanoma cell lines. Our study reveals roles for mutant BRAF zygosity and mutations in RTKs in determining basal drug resistance, and an overriding effect of expression of ERBB family RTKs in response to PLX4720 treatment. Further analysis revealed enrichment of transcription factors including ETS family members and their associated co-factors as likely regulatory drivers of adaptive PLX4720 resistance, providing a potential convergence point of adaptive resistance within the diversity of response mechanisms.

\subsection{RESULTS}

\subsubsection{Analysis overview.}

In order to gain insights into the mechanisms of synergy and sensitivity, and potentially to identify clinically relevant biomarkers, we broadly profiled our panel

of lines with multiple functional genomic and genetic assays (Figure 2.1). Analysis of the basal transcriptome revealed differences in MITF expression level that correlated weakly with drug sensitivity. Dividing the cell lines into groups based on unsupervised clustering of all the single drug and combination cytotoxic responses across a three by three dose response matrix yielded five phenotype groups. 
Strikingly, these cytotoxicity groupings closely mimicked the groupings observed in the basal transcriptome based on a principal component analysis (PCA). The transcriptional and proteomic responses to PLX4720 treatment were then analyzed to identify molecular responses that were common between the cell lines in each group. The lists of differentially expressed genes and phosphoproteins were subjected to the Mutational Signatures Database (MSigDB) (Subramanian et al., 2005) enrichment analysis and KEGG pathway enrichment analysis using Pathway Express (Draghici et al., 2007) to identify transcription factors that putatively regulate the genes in the pathways associated with response to PLX4720 and synergy to PLX4720 and lapatinib.

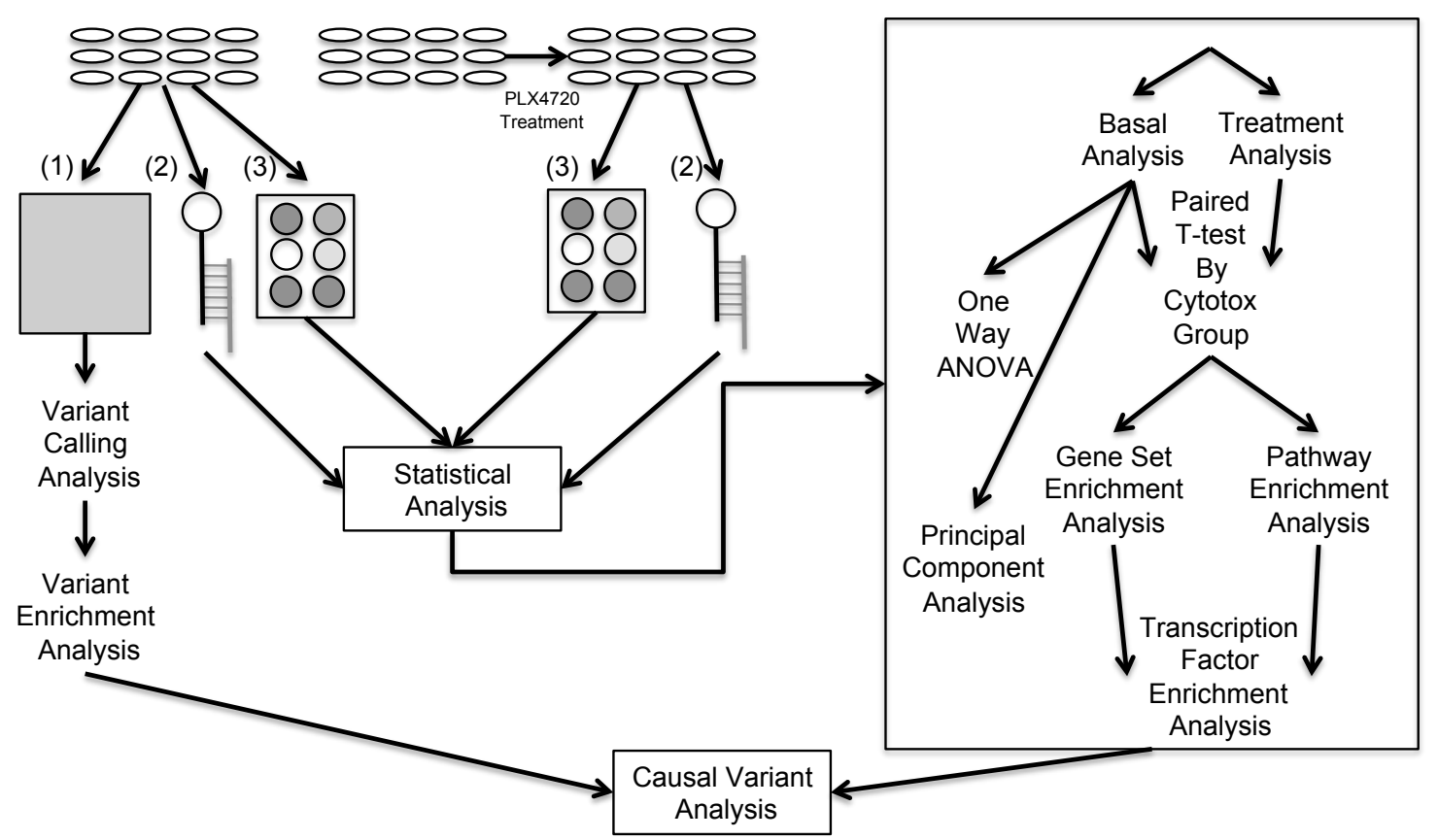

Figure 2.1 Functional genomic data generated and analysis workflow.

Flow-chart including transcriptome, proteome and exome data generated and integrative analysis of these data sets. 12 cell lines basal states were assayed using exome sequencing (1), gene expression arrays (2), and reverse phase protein arrays (3). Variant calling and enriched gene variants were identified by cross referencing our results with mutated genes associated with cancer identified by Lawrence et al (Lawrence et al., 2014). Cell lines were exposed to PLX4720 and their responses were 
assayed with gene expression arrays and reverse phase protein arrays. Protein response to treatment was correlated with cytotoxic effects of PLX4720 treatment to identify proteins that might be mitigating the cytotoxic response. Cytotoxicity (Cytotox) groups were identified by clustering the cytotoxicity data. Differential gene expression responses to treatment within each cytotoxicity group were identified and underwent both gene set and pathway enrichment analysis via MSigDB and Pathway Express respectively. Pathway enrichment analysis revealed ErbB signaling as a key response to treatment and gene set enrichment analysis revealed a number of transcription factors that are enriched which putatively regulate ErbB signaling pathway genes.

\subsubsection{Analysis of the basal transcriptome yields groupings based on MITF and drug synergy. \\ To determine whether the transcriptional profile of treatment-naïve cells}

could predict sensitivity to the drugs, singly or in combination, we classified the 12

cell lines based on unsupervised clustering of the basal transcriptome (Figure 2.2A).

Clusters I and II contained genes that were relatively high-expressed only in

SKMEL24 and VMM17, respectively, and, consequently, were not driving the ordering of the cell lines. 
A

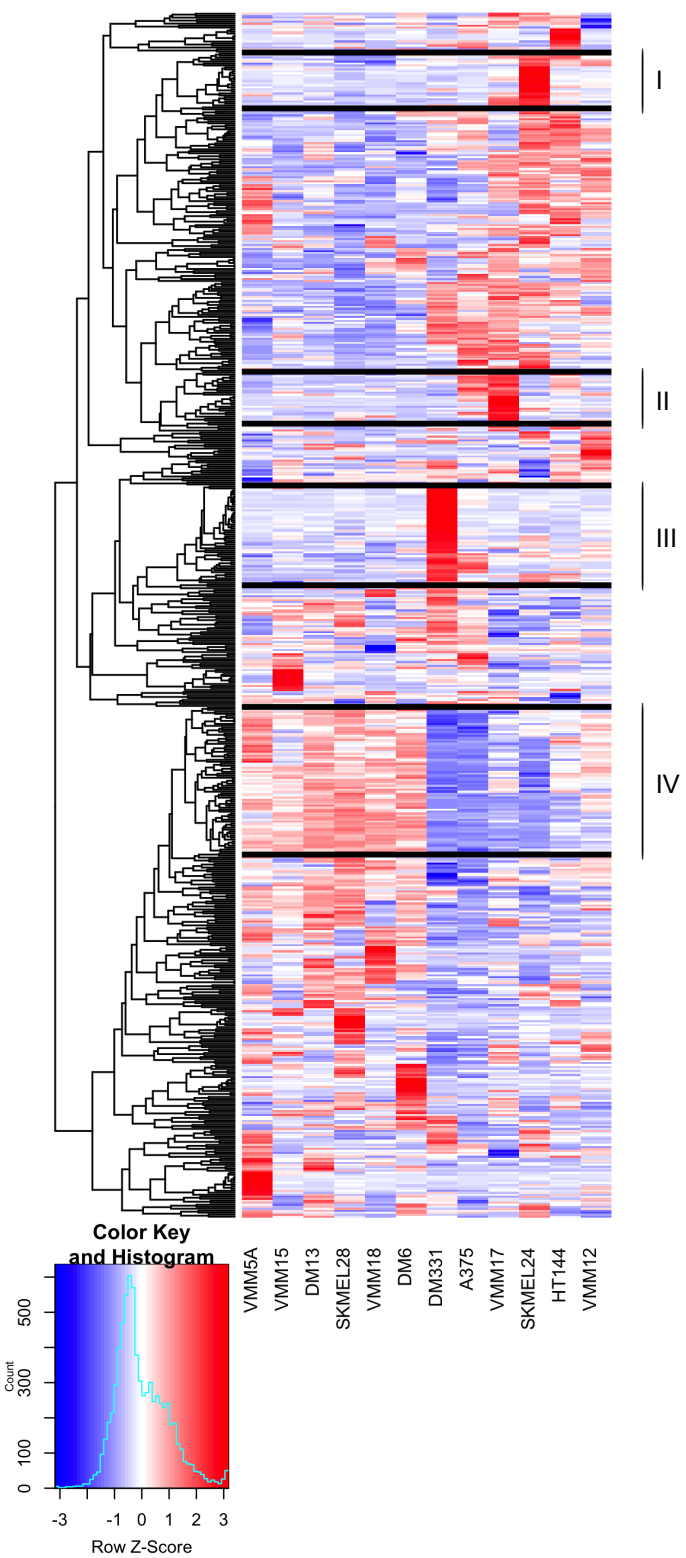

B

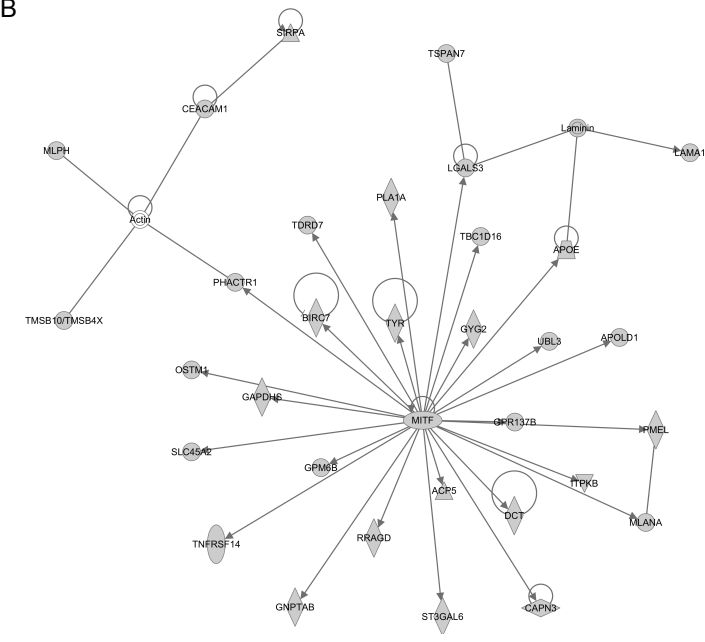

C

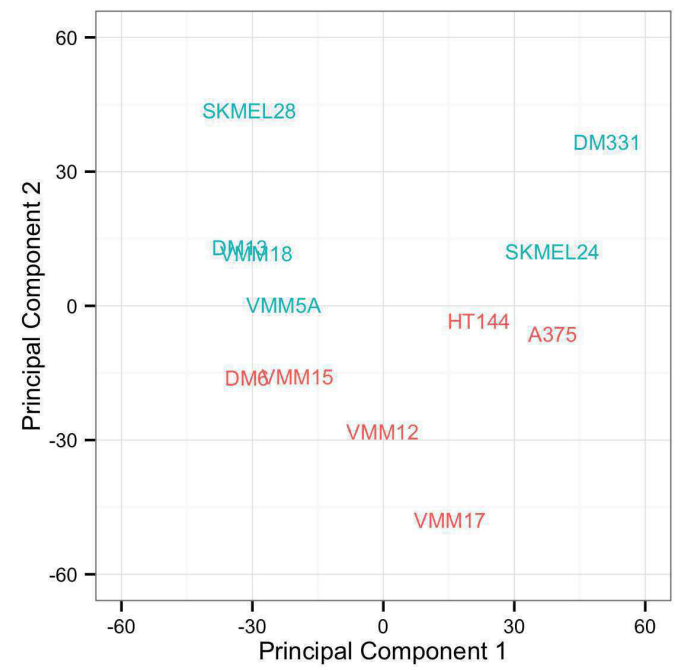

Figure 2.2 Clustering and PCA analysis of basal gene expression reveals MITF expression and gene regulation separates melanoma cell lines.

We performed a one-way analysis of variance (ANOVA) and applied a $0.1 \%$ false discovery rate (FDR) cutoff to identify genes with significantly varying expression levels, and the genes (y-axis) and cell lines ( $x$-axis) were organized by unsupervised hierarchical clustering. Clustering the genes using the correlation distance and average linkage yielded 91 gene clusters with the number of genes in each cluster ranging from 1 to 73 when applying a cluster height cutoff of 0.41. Hierarchical clustering of samples ( $x$-axis) by genes ( $y$-axis) of gene expression for significantly varying genes across twelve melanoma cell lines (A). The largest cluster (cluster IV), which contains MITF along with three others that contain cell line specific signatures (clusters I, II and III) are highlighted as they were the only clusters with greater than 
20 genes. Cluster IV genes were analyzed using Ingenuity Pathway Analysis revealing the cluster contains MITF as a regulatory hub and its target genes (B).Principal component plot of basal expression across the twelve cell lines $(C)$ separates cells according to MITF expression along the first principal component (i.e., decreasing MITF expression going from left to right) and BLISS score across the second principal component.

Cluster III (49 genes) contained relatively highly expressed genes in DM331, which is of interest because it is the most resistant line to PLX4720 treatment. A subset of the genes in this cluster was also relatively high-expressed in A375, our second most sensitive line to PLX4720 treatment. To identify the potential functional significance of the genes within cluster III, we performed a MSigDB enrichment analysis using the transcription factor gene set (Table 2.1). The top result revealed a set of 9 genes (TBL1X, IL6, CST7, NRG1, CPA4, SERPINB7, STC2, CALB2, RAB27B) that all contain the JUN motif in their promoter. Among these genes, TBL1X and CALB2 were the only two that were expressed at a relatively high level in both A375 and DM331 while the rest including NRG1 were highly expressed in DM331 only. In other works, exposure to NRG1 has been shown to induce resistance to PLX4720 treatment in A375 cells (Zhang et al., 2013). Despite the fact that DM331 and A375 are at opposite ends of the range of sensitivity to PLX4720, their transcriptional profiles are strikingly similar-placing them next to each other in the clustering of cell lines (Figure 2.2A). Taken together, these results suggest that high expression of NRG1 in DM331 contributes substantially to DM331's intrinsic resistance to PLX4720 treatment.

Table 2.1 MSigDB Table for Transcription Factor Enrichment for Clusters III and IV

\begin{tabular}{|c|c|c|c|c|}
\hline Gene Set Name & Description & $\begin{array}{c}\text { Enrichment } \\
\text { FDR q-value }\end{array}$ & Genes From Gene List In GeneSet & Cluster \\
\hline TGANTCA_V\$AP1_C & Motif TGANTCA; JUN & $1.08 \mathrm{E}-03$ & $\begin{array}{c}\text { TBL1X,IL6,CST7,NRG1,CPA4,SERPINB7,STC2,CALB2,RA } \\
\text { B27B }\end{array}$ & III \\
\hline
\end{tabular}




\begin{tabular}{|c|c|c|c|c|}
\hline $\begin{array}{l}\text { TGANNYRGCA_V\$TCF } \\
\text { 11MAFG_01 }\end{array}$ & $\begin{array}{l}\text { Motif TGANNYRGCA; } \\
\text { NFE2L1 }\end{array}$ & 4.39E-03 & TBL1X,IL6,CST7,NRG1,PPARG & III \\
\hline V\$TCF11MAFG_01 & $\begin{array}{c}\text { Motif } \\
\text { NNNNNATGACTCAGC } \\
\text { ANTTNNG; TCF11 }\end{array}$ & $1.26 \mathrm{E}-02$ & TBL1X,IL6,CST7,PPARG & III \\
\hline V\$GATA3_01 & $\begin{array}{l}\text { Motif NNGATARNG; } \\
\text { GATA3 }\end{array}$ & $1.35 \mathrm{E}-02$ & BMP4,CYB5D1,EFEMP1,KRT80 & III \\
\hline $\begin{array}{c}\text { RNGTGGGC_UNKNOW } \\
\text { N }\end{array}$ & $\begin{array}{l}\text { Motif RNGTGGGC; } \\
\text { Unknown gene }\end{array}$ & $1.35 \mathrm{E}-02$ & NRG1,CPA4,BMP4,CYB5D1,TLE4,NMU & III \\
\hline V\$IPF1_Q4 & $\begin{array}{c}\text { Motif } \\
\text { GHNNTAATGACM; } \\
\text { IPF1 }\end{array}$ & $1.35 \mathrm{E}-02$ & BMP4,EFEMP1,TLE4,RNF212 & III \\
\hline V\$NFAT_Q4_01 & $\begin{array}{c}\text { Motif } \\
\text { NWGGAAANWN; } \\
\text { NFAT }\end{array}$ & $1.35 \mathrm{E}-02$ & SERPINB7,STC2,PTHLH,SOX9 & III \\
\hline V\$NFE2_01 & $\begin{array}{l}\text { Motif TGCTGAGTCAY; } \\
\text { NFE2 }\end{array}$ & $1.35 \mathrm{E}-02$ & TBL1X,IL6,CST7,CALB2 & III \\
\hline CTTTGA_V\$LEF1_Q2 & Motif CTTTGA; LEF1 & $1.65 \mathrm{E}-02$ & NRG1,PPARG,TLE4,PTHLH,SLC14A1,UCP2,COL5A1 & III \\
\hline V\$PBX1_02 & $\begin{array}{c}\text { Motif } \\
\text { NNCATCAATCAANNW } \\
; \text { PBX1 }\end{array}$ & $1.83 \mathrm{E}-02$ & TBL1X,TLE4,PTHLH & III \\
\hline TATAAA_V\$TATA_01 & Motif TATAAA; TAF & $1.83 \mathrm{E}-02$ & CPA4,PPARG,EFEMP1,TLE4,PTHLH,SOX9,CD24 & III \\
\hline $\begin{array}{l}\text { TGGAAA_V\$NFAT_Q4 } \\
\text { _01 }\end{array}$ & Motif TGGAAA; NFAT & $3.17 E-02$ & TBL1X,IL6,NRG1,SERPINB7,BMP4,CYB5D1,SOX9,ALPK2 & III \\
\hline CAGGTG_V\$E12_Q6 & Motif CAGGTG; TCF3 & $3.87 \mathrm{E}-02$ & $\begin{array}{l}\text { NRG1,CPA4,STC2,BMP4,EFEMP1,TLE4,PTHLH,FZD2,SC } \\
\text { G5 }\end{array}$ & III \\
\hline $\begin{array}{c}\text { TCANNTGAY_V\$SREBP } \\
\text { 1_01 } \\
\end{array}$ & $\begin{array}{c}\text { Motif TCANNTGAY; } \\
\text { SREBF1 } \\
\end{array}$ & $6.71 \mathrm{E}-03$ & PMEL,TRIM63,WDFY1,CAPN3,TYR,SLC24A5,CYP27A1 & IV \\
\hline V\$CEBPA_01 & $\begin{array}{c}\text { Motif } \\
\text { NNATTRCNNAANNN; } \\
\text { CEBPA } \\
\end{array}$ & $1.39 \mathrm{E}-02$ & MITF,SIRPA,GYPC,PROS1,TBC1D16 & IV \\
\hline TTGTTT_V\$FFXXO4_01 & Motif TTGTTT; MLLT7 & $1.56 \mathrm{E}-02$ & $\begin{array}{l}\text { PMEL,TRIM63,MITF,SIRPA,GHR,APOLD1,GPR137B,DCT } \\
\text {,DAAM2,IGFBP1,UBL3,MLANA }\end{array}$ & IV \\
\hline CAGCTG_V\$AP4_Q5 & $\begin{array}{l}\text { Motif CAGCTG; } \\
\text { REPIN1 }\end{array}$ & $1.56 \mathrm{E}-02$ & $\begin{array}{l}\text { PMEL,WDFY1,MITF,GHR,APOLD1,GPM6B,RRAGD,MBP, } \\
\text { BCAS3,LONRF1 }\end{array}$ & IV \\
\hline $\begin{array}{l}\text { RGAGGAARY_V\$PU1_ } \\
\text { Q6 }\end{array}$ & $\begin{array}{l}\text { Motif RGAGGAARY; } \\
\text { SPI1 }\end{array}$ & $1.56 \mathrm{E}-02$ & TRIM63,CAPN3,GPR137B,NAV2,GPR56,NDN & IV \\
\hline TGCCAAR_V\$NF1_Q6 & Motif TGCCAAR; NF1 & $1.56 \mathrm{E}-02$ & MITF,GHR,DCT,TSPAN7,PI15,GJA3,ADCY1 & IV \\
\hline $\begin{array}{c}\text { GGGAGGRR_V\$MAZ_ } \\
\text { Q6 }\end{array}$ & $\begin{array}{l}\text { Motif GGGAGGRR; } \\
\text { MAZ }\end{array}$ & $1.63 \mathrm{E}-02$ & $\begin{array}{l}\text { MITF,GYPC,GPR137B,GPM6B,NAV2,GPR56,TSPAN7,PI1 } \\
\text { 5,SLC27A3,BHLHE41,LAMA1,GDF15 }\end{array}$ & IV \\
\hline V\$ATF_B & $\begin{array}{c}\text { Motif } \\
\text { NTGACGTCANYS; } \\
\text { Unknown gene }\end{array}$ & $1.63 \mathrm{E}-02$ & MITF,DAAM2,GPM6B,TSPAN7 & IV \\
\hline $\begin{array}{l}\text { RGTTAMWNATT_V\$H } \\
\text { NF1_01 }\end{array}$ & $\begin{array}{c}\text { Motif } \\
\text { RGTTAMWNATT; } \\
\text { TCF1 }\end{array}$ & $1.63 \mathrm{E}-02$ & MITF,IGFBP1,PLA1A & IV \\
\hline $\begin{array}{c}\text { TGACGTCA_V\$ATF3_Q } \\
\frac{6}{4}\end{array}$ & $\begin{array}{l}\text { Motif TGACGTCA; } \\
\text { ATF3 }\end{array}$ & 2.67E-02 & MITF,DAAM2,GPM6B,TSPAN7 & IV \\
\hline V\$PU1_Q6 & $\begin{array}{c}\text { Motif WGAGGAAG; } \\
\text { SPI1 } \\
\end{array}$ & 2.67E-02 & SIRPA,NAV2,NDN,SLC27A3 & IV \\
\hline V\$HNF1_01 & $\begin{array}{c}\text { Motif } \\
\text { GGTTAATNWTTAMC } \\
\text { N; TCF1 } \\
\end{array}$ & 2.67E-02 & MITF,IGFBP1,PLA1A,DAB2 & IV \\
\hline $\begin{array}{c}\text { GGGYGTGNY_UNKNO } \\
\text { WN }\end{array}$ & $\begin{array}{l}\text { Motif GGGYGTGNY; } \\
\text { Unknown gene }\end{array}$ & 2.67E-02 & MITF,APOLD1,UBL3,NAV2,PI15,LGALS3 & IV \\
\hline V\$ATF3_Q6 & $\begin{array}{c}\text { Motif } \\
\text { CBCTGACGTCANCS; } \\
\text { ATF3 } \\
\end{array}$ & 2.67E-02 & MITF,DAAM2,GPM6B,TSPAN7 & IV \\
\hline GGGCGGR_V\$SP1_Q6 & Motif GGGCGGR; SP1 & 2.67E-02 & $\begin{array}{l}\text { PMEL,MITF,SIRPA,GYPC,PROS1,DAAM2,GPM6B,NDN,S } \\
\text { LC27A3,BHLHE41,GJB1,APOE,MLPH }\end{array}$ & IV \\
\hline V\$ATF_01 & $\begin{array}{c}\text { Motif } \\
\text { CNSTGACGTNNNYC; } \\
\text { Unknown gene }\end{array}$ & 2.67E-02 & PMEL,DAAM2,GPM6B,TSPAN7 & IV \\
\hline
\end{tabular}




\begin{tabular}{|c|c|c|c|c|}
\hline V\$̦CREBP1CJUN_01 & Motif TGACGTYA; JUN & $2.67 \mathrm{E}-02$ & MITF,DAAM2,GPM6B,TSPAN7 & IV \\
\hline V\$NF1_Q6 & $\begin{array}{c}\text { Motif } \\
\text { NNTTGGCNNNNNNC } \\
\text { CNNN; NF1 }\end{array}$ & 2.67E-02 & TYR,DCT,NAV2,GJA3 & IV \\
\hline V\$CREB_01 & $\begin{array}{c}\text { Motif TGACGTMA; } \\
\text { CREB1 }\end{array}$ & 2.67E-02 & MITF,DAAM2,GPM6B,TSPAN7 & IV \\
\hline V\$NF1_Q6_01 & $\begin{array}{c}\text { Motif } \\
\text { NTGGNNNNNNGCCA } \\
\text { ANN; NF1 }\end{array}$ & $2.74 \mathrm{E}-02$ & TYR,DCT,NAV2,GJA3 & IV \\
\hline V\$GR_Q6 & $\begin{array}{c}\text { Motif } \\
\text { NNNNNNCNNTNTGT } \\
\text { NCTNN; NR3C1 }\end{array}$ & $2.74 \mathrm{E}-02$ & MITF,DAAM2,RRAGD,MBP & IV \\
\hline V\$AR_Q2 & $\begin{array}{c}\text { Motif } \\
\text { AGWACATNWTGTTCT } \\
; \text { AR } \\
\end{array}$ & 2.97E-02 & APOLD1,DAB2,GJB1 & IV \\
\hline TGACCTY_V\$ERR1_Q2 & $\begin{array}{c}\text { Motif TGACCTY; } \\
\text { ESRRA }\end{array}$ & $3.61 \mathrm{E}-02$ & MITF,SIRPA,DCT,UBL3,RRAGD,TSPAN7,ADCY1 & IV \\
\hline $\begin{array}{c}\text { TGAYRTCA_V\$ATF3_Q } \\
\frac{6}{4}\end{array}$ & $\begin{array}{c}\text { Motif TGAYRTCA; } \\
\text { ATF3 }\end{array}$ & 4.32E-02 & MITF,DAAM2,GPM6B,TSPAN7,GAPDHS & IV \\
\hline TGANTCA_V\$SAP1_C & Motif TGANTCA; JUN & 4.99E-02 & MITF,DCT,BCAS3,GPR56,PI15,LGALS3,GAPDHS & IV \\
\hline
\end{tabular}

Clustering the expression profile of the 73 genes in Cluster IV separates our cell lines into two distinct groups. When we inspect the content of the lines present in either the right or left side of Cluster IV, the separation does not appear to be driven by the lines synergy status (Figure 2.3). Ingenuity Pathway analysis of this list of 73 genes revealed that cluster IV contained MITF as a hub with a number of MITF target genes (Figure 2.2B) suggesting that the cell lines could be in different cell lineage states with the MITF transcriptional program driving this separation. To confirm whether MITF is a regulatory hub of cluster IV genes, we compared the genes in cluster IV with MITF target genes identified by Strub et. al. via a ChIP-Seq experiment (Strub et al., 2011) and observed an overlap of 33 of 73 (45\%; p-value = 3.42 e-11; Figure 2.4). These results implicate MITF and the expression of its associated targets as potentially driving lineage separation in our cell lines, but not contributing consistently to drug sensitivity. 


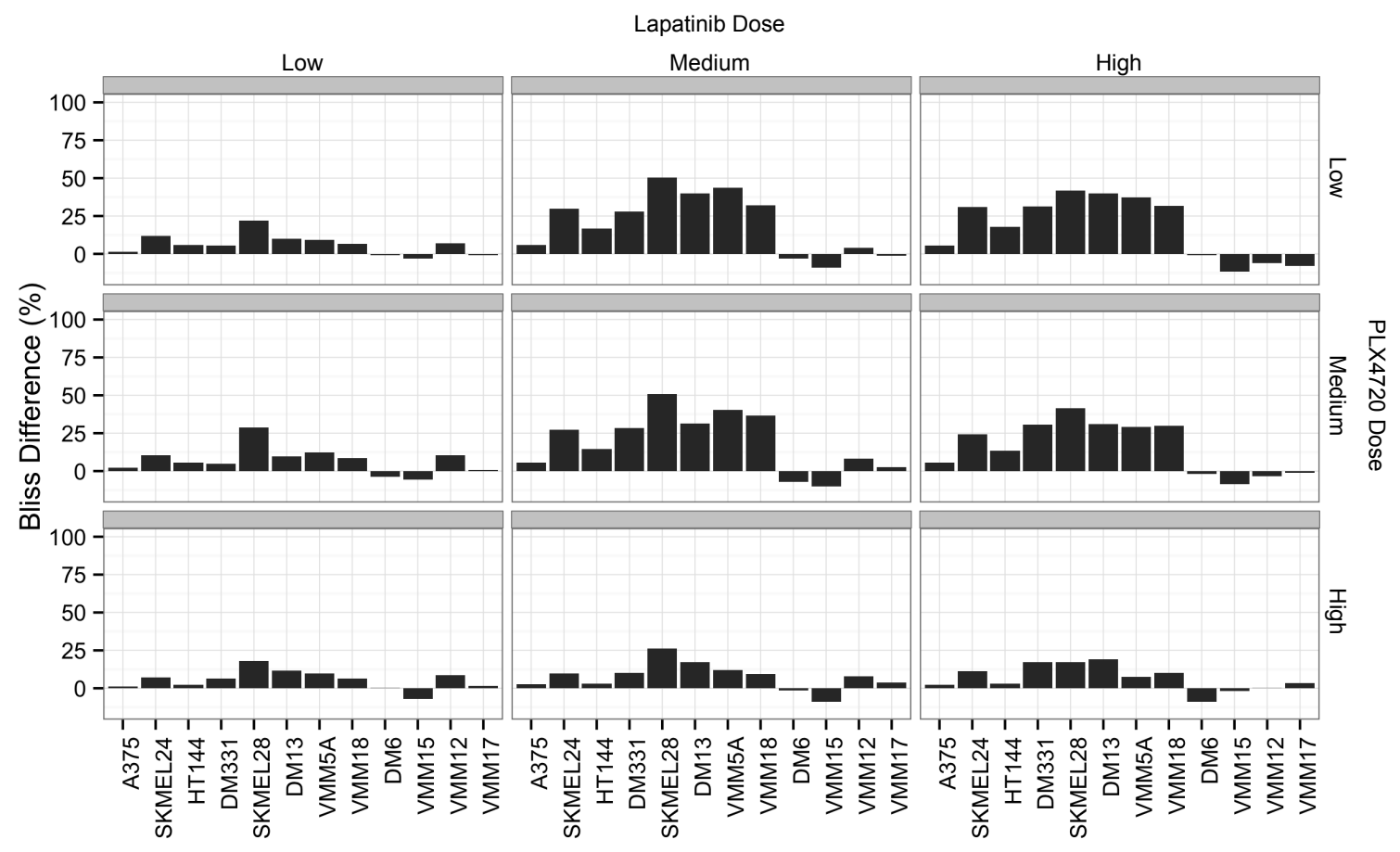

Figure 2.3 Bliss difference (Synergy score) for lines across 3 x 3 dose response

Synergy score (left y-axis) across all 12 cell lines (bottom $x$-axis) for escalating doses of lapatinib (top $x$-axis) and PLX4720 (righty-axis). Synergy Score was calculated using the Bliss model of independence. Synergy Score $=C_{\text {Combination }}-\left(1-\left(1-C_{\text {Secondary }}\right.\right.$ Drug $^{*}\left(1-C_{\text {Primary Drug }}\right)$ ) where $C_{\text {Combination }}$ is the observed cytotoxicity of the combination treatment, $C_{\text {Secondary Drug }}$ is the observed cytotoxicity of the secondary drug, and $C_{\text {Primary }}$ Drug is the observed cytotoxicity of the primary drug. 


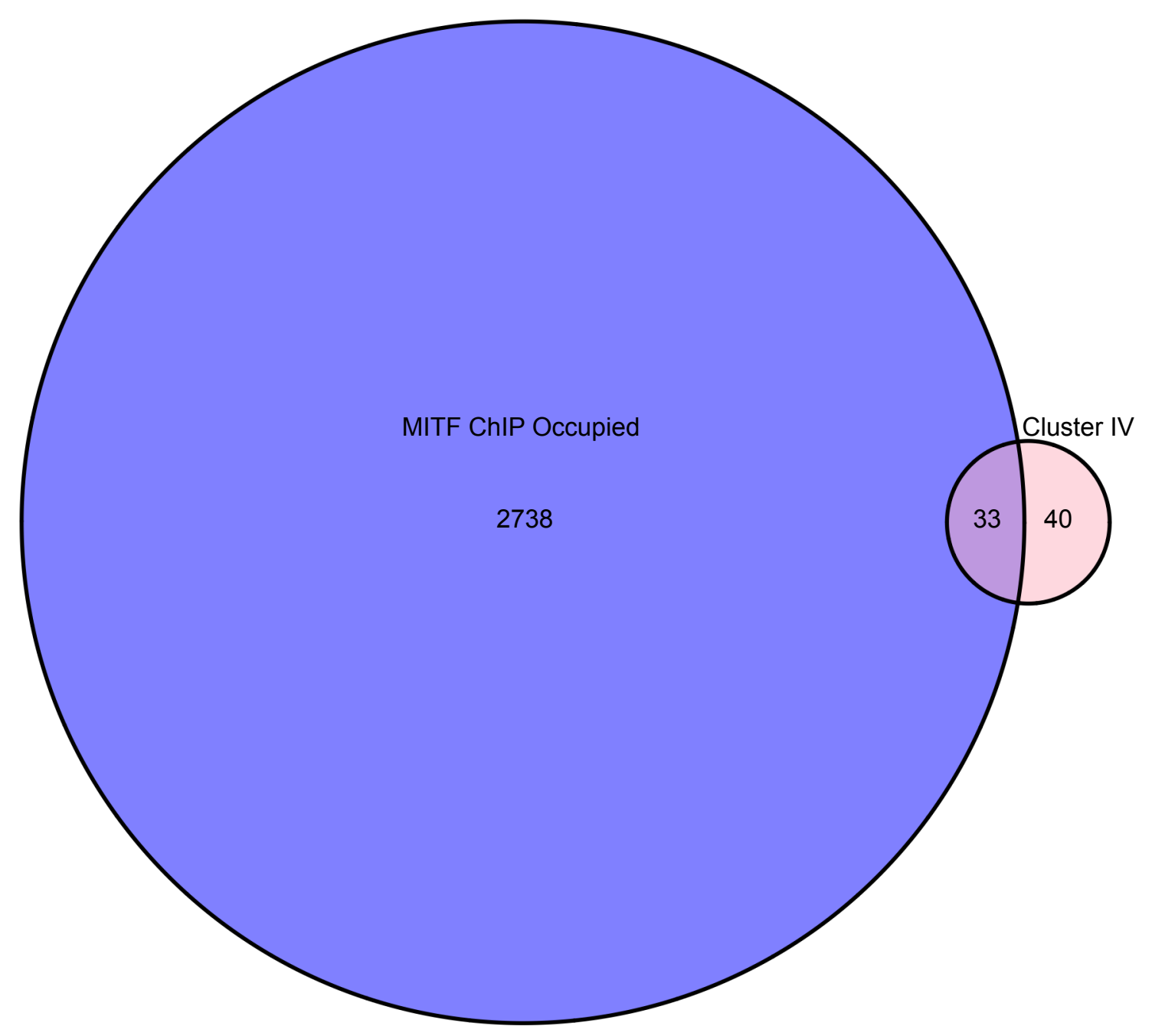

Figure 2.4 Overlap of Cluster IV genes with genes that contain MITF binding sites as identified by ChIP Seq

Number of overlapping genes (purple) between MITF ChIP Seq binding sites (blue) and Cluster IV gene list (pink)

To assess the extent to which the MITF expression is associated with

response to PLX4720 treatment in our panel, we correlated MITF expression in each of our lines with their respective IC50 values. We observed a modest anticorrelation of -0.40 between the MITF expression level of our lines, and their respective IC50 doses, consistent with Konieczkowski et al. (Konieczkowski et al., 2014). In our case, 
this result is largely driven by DM331, our most resistant line. When this line is excluded, we observe essentially no correlation, 0.085 , between MITF gene expression and IC50 across the remaining eleven cell lines. Because our synergy studies were performed over a dose range that included concentrations below the IC50 (centered around IC20), we also examined the correlation of MITF expression and cytotoxicity at the low, medium and high doses. We found a relatively strong anticorrelation between MITF gene expression level and PLX4720 cytotoxicity (excluding DM331): $-0.79,-0.68$ and -0.67 for our low, medium and high doses, respectively. Notably, our PLX4720 doses, which were intended to assess drug synergy, are much lower, $125 \mathrm{nM}$, in our studies compared to other studies which tend use $\sim 1 \mu \mathrm{M}$ PLX4720 (Halaban et al., 2009; Konieczkowski et al., 2014; Müller et al., 2014; Søndergaard et al., 2010). Thus, at low doses of PLX4720, we find lower levels of MITF across our cell lines are associated with increased sensitivity to PLX4720 treatment while at IC50 doses, we find essentially no correlation.

To analyze mechanisms of synergy between PLX4720 and lapatinib we performed a principal components analysis (PCA) which reveals dominant sources of variability in the first principal component as well as sub-dominant sources of variability in the second and higher order principal components. As shown in the PCA plot (Figure 2.2C), the separation along the first principal component (PC1) was not associated with synergy, but appeared to be strongly correlated with MITF expression level in our lines (correlation coefficient $=-0.9$ ). We observed an inverse relationship between MITF expression level and AXL kinase expression level (Figure 2.5), a trend which has been observed previously (Johannessen et al., 2013; 
Konieczkowski et al., 2014). The separation that we observed along the second principal component (PC2) segregated synergy and non-synergy cell lines, and was highly correlated with the synergy score of the lines (correlation coefficient = $0.791)$. 
A

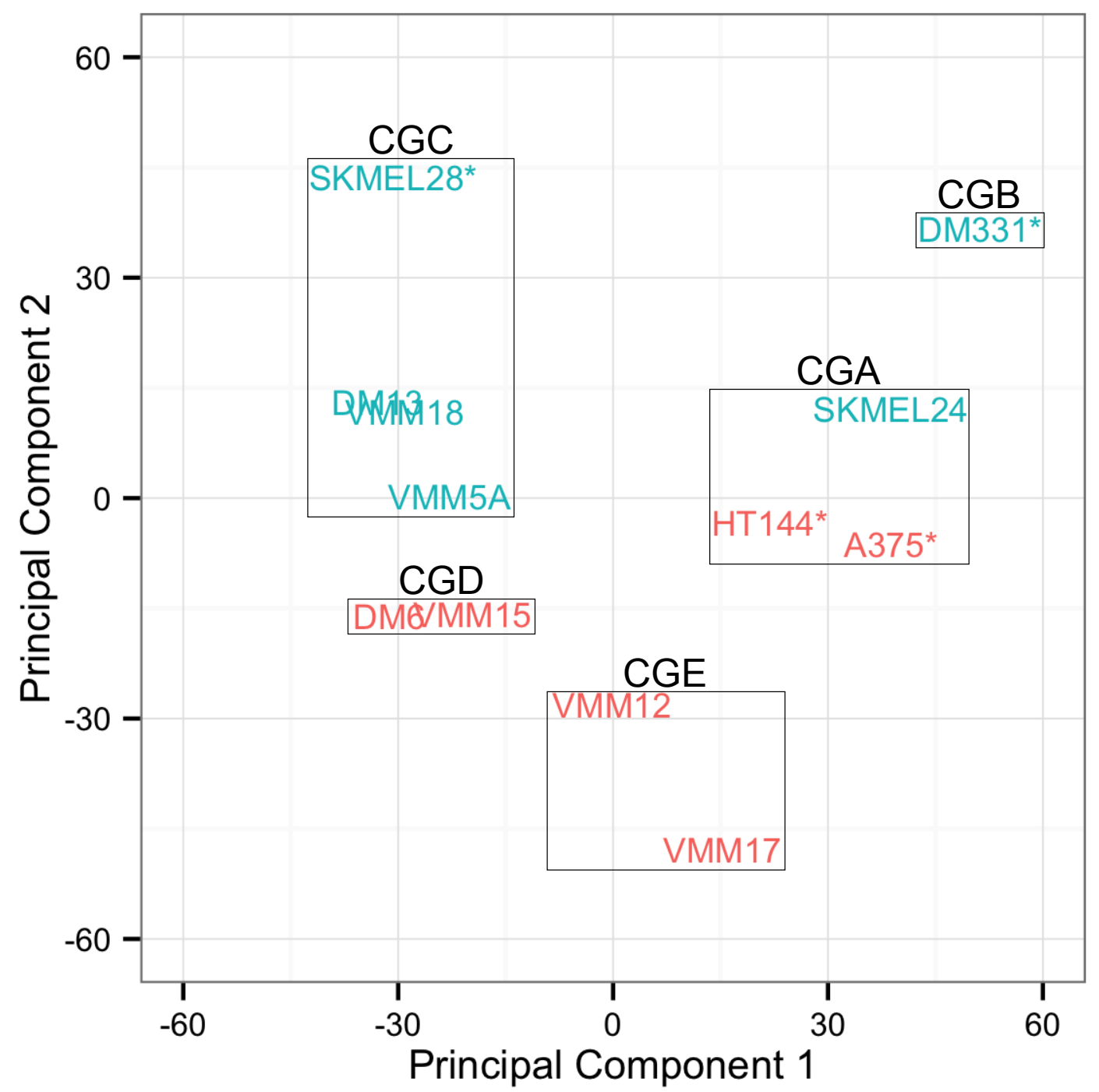

B

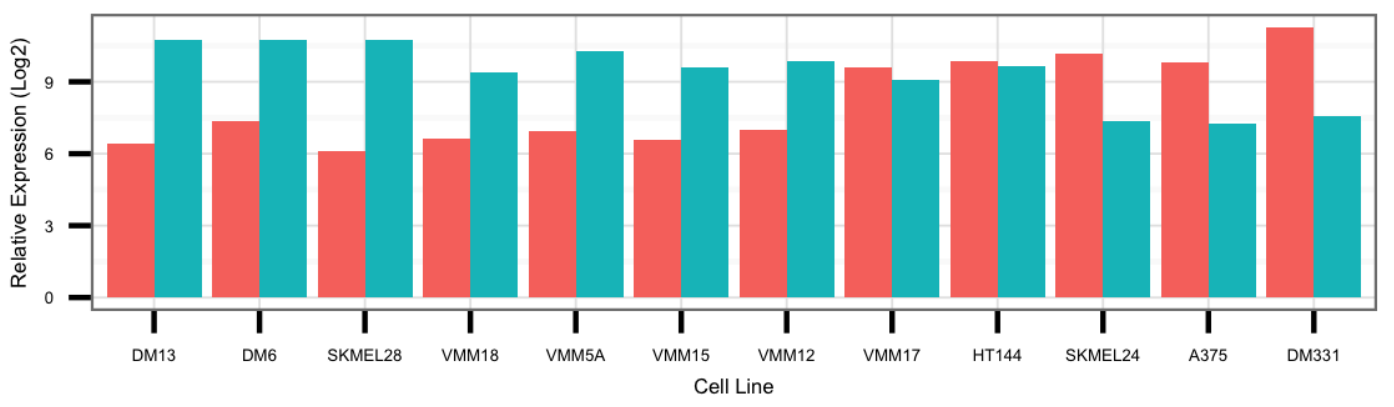

Figure 2.5 Cytotoxicty groupings are strongly associated with PCA loadings

Basal PCA plot with cytotoxicity groups encircled, and homozygous BRAF mutants denoted with a "*” (A). Relative expression in Log2 of AXL (Red) and MITF (Blue) (B). 
The strong correlation of the second principal component with the Bliss synergy score of the cell lines raised the possibility that the basal transcriptional profile could yield insights into mechanisms of synergy and resistance. To explore this possibility, we correlated each gene expression profile across cell lines with PC2 and identified pathways enriched for these genes using MSigDB enrichment analyses (Subramanian et al., 2005). We input genes whose correlation with PC2 was greater than 0.8 or less than -0.8 . Pathways that were enriched with genes that were highly correlated with PC2 (Table 2.2) were cell cycle and GPCR signaling associated pathways. Pathways that were enriched with genes that were highly anticorrelated with PC2 also included GPCR signaling, neuroligand GPCRs and cytokine-cytokine receptor interaction.

Table 2.2 Top 10 pathways from MSigDB enrichment analysis for genes that have a negative or positive correlation coefficient with the second principal component

\begin{tabular}{|c|c|c|}
\hline Gene Set Name & Description & $\begin{array}{c}\text { Correlation } \\
\text { Status with PC2 }\end{array}$ \\
\hline REACTOME_GPCR_DOWNSTREAM_SIGNALING & $\begin{array}{l}\text { Genes involved in } \\
\text { GPCR downstream } \\
\text { signaling }\end{array}$ & Negative \\
\hline REACTOME_SIGNALING_BY_GPCR & $\begin{array}{l}\text { Genes involved in } \\
\text { Signaling by GPCR }\end{array}$ & Negative \\
\hline KEGG_OLFACTORY_TRANSDUCTION & $\begin{array}{c}\text { Olfactory } \\
\text { transduction } \\
\end{array}$ & Negative \\
\hline REACTOME_OLFACTORY_SIGNALING_PATHWAY & $\begin{array}{c}\text { Genes involved in } \\
\text { Olfactory Signaling } \\
\text { Pathway }\end{array}$ & Negative \\
\hline REACTOME_GPCR_LIGAND_BINDING & $\begin{array}{c}\text { Genes involved in } \\
\text { GPCR ligand binding }\end{array}$ & Negative \\
\hline KEGG_NEUROACTIVE_LIGAND_RECEPTOR_INTERACTION & $\begin{array}{l}\text { Neuroactive ligand- } \\
\text { receptor interaction }\end{array}$ & Negative \\
\hline REACTOME_CLASS_A1_RHODOPSIN_LIKE_RECEPTORS & $\begin{array}{c}\text { Genes involved in } \\
\text { Class A/1 } \\
\text { (Rhodopsin-like } \\
\text { receptors) }\end{array}$ & Negative \\
\hline KEGG_CYTOKINE_CYTOKINE_RECEPTOR_INTERACTION & $\begin{array}{l}\text { Cytokine-cytokine } \\
\text { receptor interaction }\end{array}$ & Negative \\
\hline REACTOME_NEURONAL_SYSTEM & $\begin{array}{l}\text { Genes involved in } \\
\text { Neuronal System }\end{array}$ & Negative \\
\hline REACTOME_IMMUNE_SYSTEM & $\begin{array}{l}\text { Genes involved in } \\
\text { Immune System }\end{array}$ & Negative \\
\hline REACTOME_CELL_CYCLE & $\begin{array}{c}\text { Genes involved in } \\
\text { Cell Cycle }\end{array}$ & Positive \\
\hline
\end{tabular}




\begin{tabular}{|c|c|c|}
\hline REACTOME_CELL_CYCLE_MITOTIC & $\begin{array}{l}\text { Genes involved in } \\
\text { Cell Cycle, Mitotic }\end{array}$ & Positive \\
\hline REACTOME_TRANSMEMBRANE_TRANSPORT_OF_SMALL_MOLECULES & $\begin{array}{l}\text { Genes involved in } \\
\text { Transmembrane } \\
\text { transport of small } \\
\text { molecules }\end{array}$ & Positive \\
\hline KEGG_OLFACTORY_TRANSDUCTION & $\begin{array}{c}\text { Olfactory } \\
\text { transduction }\end{array}$ & Positive \\
\hline REACTOME_IMMUNE_SYSTEM & $\begin{array}{l}\text { Genes involved in } \\
\text { Immune System }\end{array}$ & Positive \\
\hline REACTOME_ANTIGEN_PROCESSING_UBIQUITINATION_PROTEASOME_DEGRADATION & $\begin{array}{c}\text { Genes involved in } \\
\text { Antigen processing: } \\
\text { Ubiquitination \& } \\
\text { Proteasome } \\
\text { degradation } \\
\end{array}$ & Positive \\
\hline REACTOME_SIGNALING_BY_GPCR & $\begin{array}{l}\text { Genes involved in } \\
\text { Signaling by GPCR }\end{array}$ & Positive \\
\hline REACTOME_S_PHASE & $\begin{array}{c}\text { Genes involved in } \mathrm{S} \\
\text { Phase }\end{array}$ & Positive \\
\hline REACTOME_ADAPTIVE_IMMUNE_SYSTEM & $\begin{array}{c}\text { Genes involved in } \\
\text { Adaptive Immune } \\
\text { System }\end{array}$ & Positive \\
\hline REACTOME_GPCR_DOWNSTREAM_SIGNALING & $\begin{array}{l}\text { Genes involved in } \\
\text { GPCR downstream } \\
\text { signaling }\end{array}$ & Positive \\
\hline
\end{tabular}

\subsubsection{Grouping Cell Lines According to their Cytotoxicity Profiles is Consistent with Their Transcriptome-based Grouping}

Given the modest association between basal gene expression and synergy, we wanted to determine if there was a phenotypic classification that better correlated with the basal gene expression analyses. We re-examined the grouping of the cell lines according to cytotoxic drug response over the low dose ranges used to identify synergy. These analyses of cytotoxic responses to PLX4720, lapatinib and the combination of the two drugs revealed that the lines that did not demonstrate synergy had low synergy scores for fundamentally different reasons (detailed below, Figure 2.3). To organize the cell lines according to drug cytotoxicity profiles, we clustered the cell lines using their cytotoxicity values across drug treatments (Figure 2.6A), again applying the Pearson correlation distance and average linkage. This cytotoxicity based clustering yielded five distinct phenotypic groups. The clustering of cell lines' cytotoxic responses revealed a strong separation between 
the synergistic lines and the non synergistic lines with the exception of SKMEL24, a synergistic line which grouped with the non synergistic lines. To verify the robustness of the clustering in which averages of cytotoxicity values across replicates were used, we generated box plots of all the cytotoxicity values (Figure 2.7). Both the trends with drug dose and actual cytotoxicity values were robustly similar across cell lines within a cytotoxic group and noticeably distinct between groups. Strikingly, cell lines within cytotoxic groups clustered according to their basal transcriptome-based grouping in the principal component plot (Figure 2.5). The five groups identified were: (1) Cytotoxicity group A (CGA), whose cells show strong dose dependence on and relatively high cytotoxicity in response to PLX4720 treatment while lapatinib treatment is static across all doses, and its cytotoxicity is moderate; (2) Cytotoxicity group B (CGB), which only contains DM331. This cell line is almost completely resistant to PLX4720, and has relatively high expression of NRG1, a known mechanism of PLX4720 resistance (Zhang et al., 2013). For this reason we exclude it from further transcriptional group analyses; (3) Cytotoxicity group C (CGC), in which cells respond to the drug combination but are resistant to either drug alone; (4) Cytotoxicity group D (CGD), in which cells grow in the presence of low dose lapatinib and have reduced response to combination at low doses compared to other groups; and (5) Cytotoxicity group E (CGE), whose cells grow in the presence of low dose lapatinib. 
A
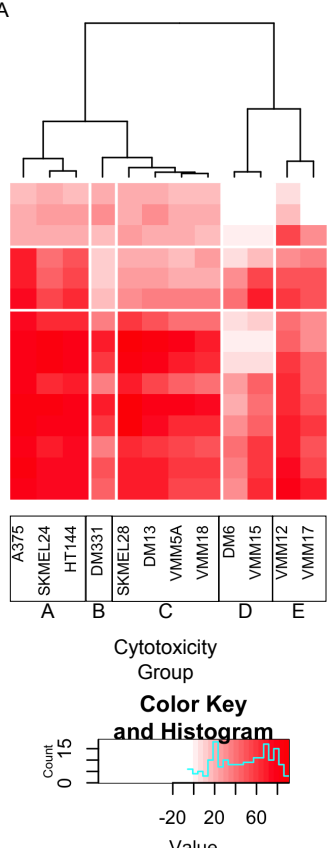
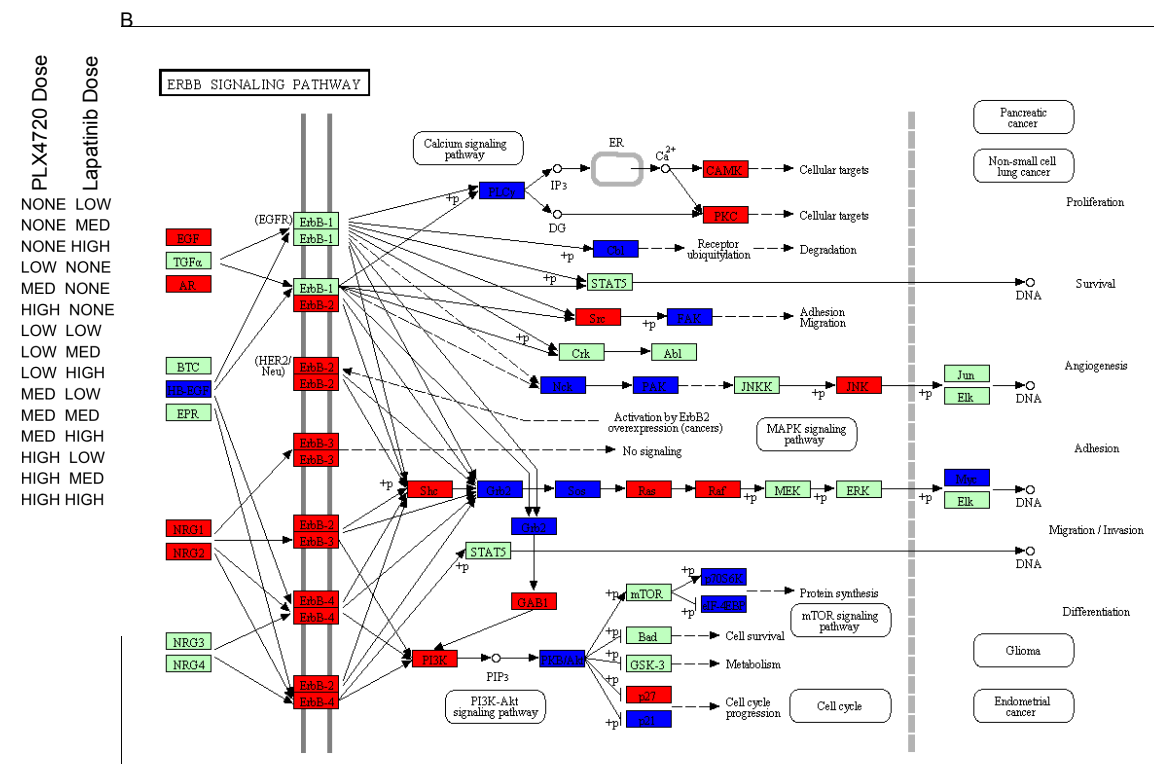

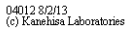

Figure 2.6 Clustering by cytotoxicity reveals differential responses to PLX4720 treatment.

Clustering of cell lines ( $x$-axis) according to cytotoxic responses to PLX4720, lapatinib, and combination treatment at three doses for each individual drug and a three by three dose response for the combination (y-axis) (A). Five groups were identified: CGA (most left, 3 lines), CGB (near left, 1 line), CGC (center, 4 lines), CGD (near right, 2 lines), and CGE (far right, 2 lines). Pathway enrichment analysis of differential gene expression to PLX4720 treatment identified ErbB signaling as being broadly activated in $C G C(B)$. 


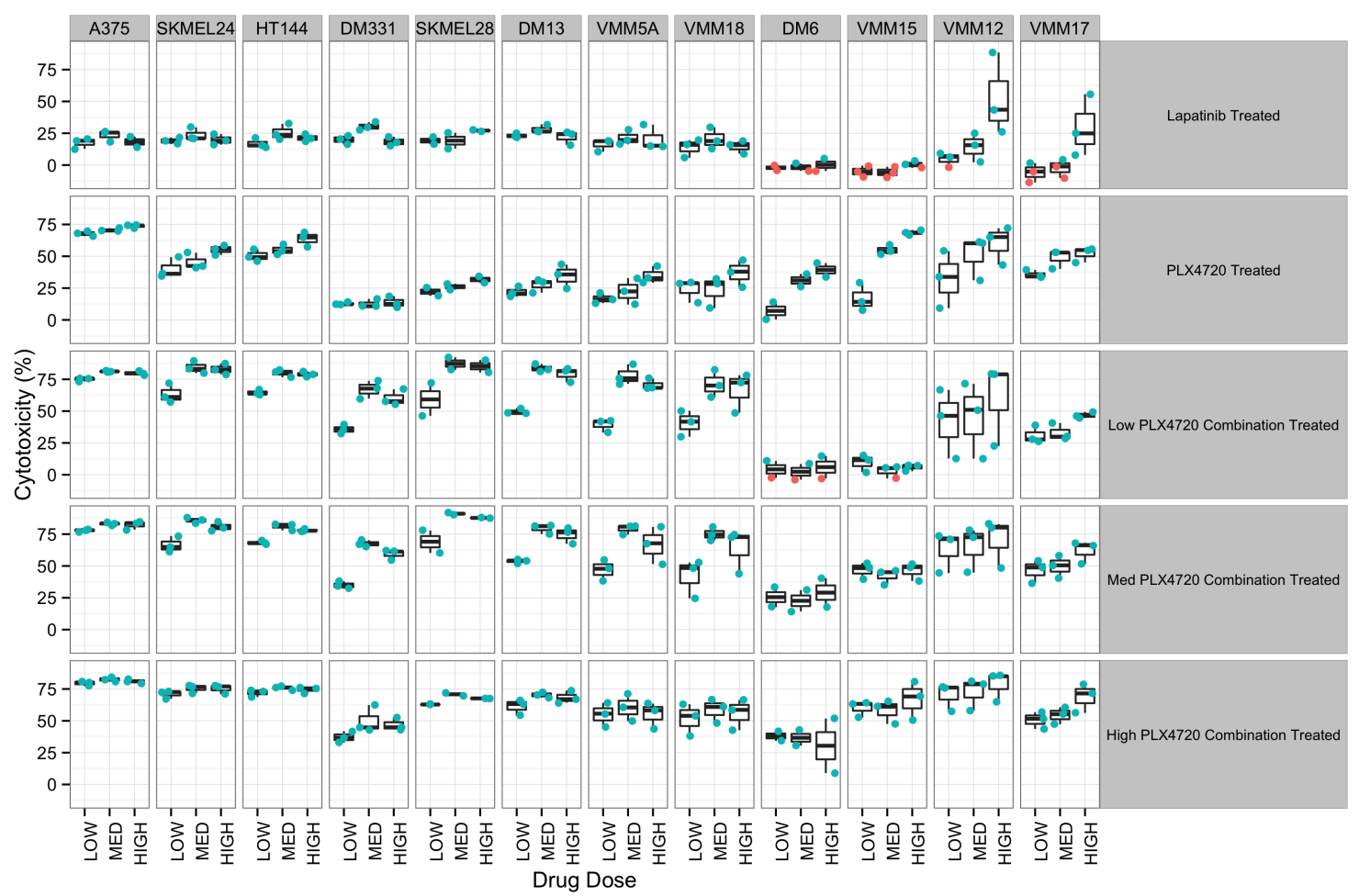

Figure 2.7 Cytotoxic responses to single and combination drug treatments for cell lines

Box plots of cytotoxic response to single drug treatment (top 2 rows) and escalating PLX4720 doses of the combination drug treatment (bottom 3 rows). Doses along the bottom refer to either the single drug treatment (top 2 rows), or the secondary drug treatment dose for the combination drug treatment (bottom 3 rows). Red dots denote negative cytotoxic responses, blue dots denote positive cytotoxic responses.

\subsubsection{Exome Sequencing Reveals Mutations That Modulate Lineage-Based Cytotoxicity Profiles}

To determine whether the mutational landscape of the cell lines could explain their grouping according to cytotoxicity profiles, we performed whole exome sequencing on all twelve cell lines. We found a trend between the zygosity status of the BRAF locus and response to treatment, with homozygous BRAF mutant lines being more sensitive to BRaf inhibition, as has previously been reported in a panel of cell lines by Søndergaard et al (Søndergaard et al., 2010). The only overlapping line between their study and ours was SKMEL28, and we confirm the mutations that they observed. To expand this analysis, we obtained IC50 values to 
PLX4720 for 27 BRAFV600E melanoma cell lines in the Cancer Cell Line Encyclopedia (Barretina et al., 2012) and plotted the IC50 for each line in order, as well as lines in our study, and colored the bars based on their zygosity status at the BRAF locus (Figure 2.8A). We observed a trend between the zygosity and IC50, with five out of six homozygous lines located in the left (more sensitive) half of the chart. The only outlier was DM331, which we again excluded from our analysis. We obtained a pvalue $=0.049$ using a permutation-based t-test comparing IC50 values of homozygous versus heterozygous lines. We then generated box plots for the IC50 values for BRAFV600E/V600E melanomas, BRAFWT/N600E melanomas and BRAFWT/WT melanomas. We observed a clear trend between the zygosity status and the sensitivity to PLX4720 treatment (Figure 2.8B), similar to the findings of Halaban et al. (Halaban et al., 2010). Lastly, we note that our study has a much larger percentage of BRAFV600E/V600E melanomas compared to other studies, which could explain why BRAF zygosity had a stronger association with sensitivity to PLX4720 treatment in our study compared to others. 
A
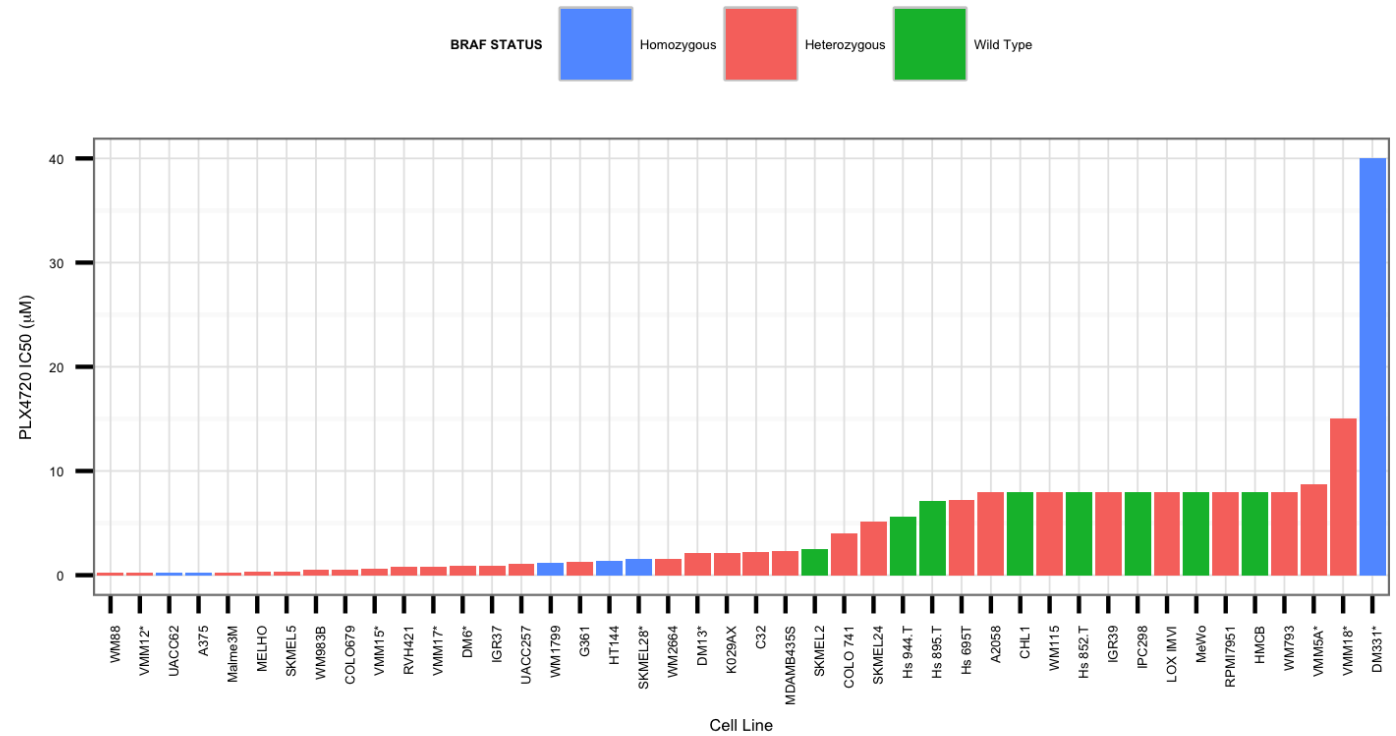

B

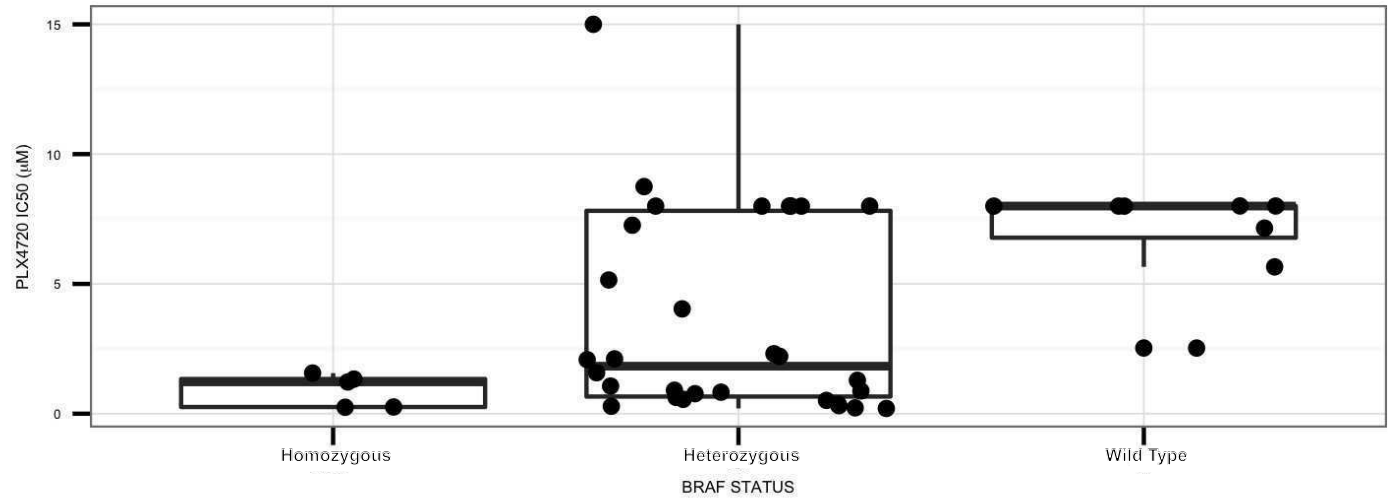

C
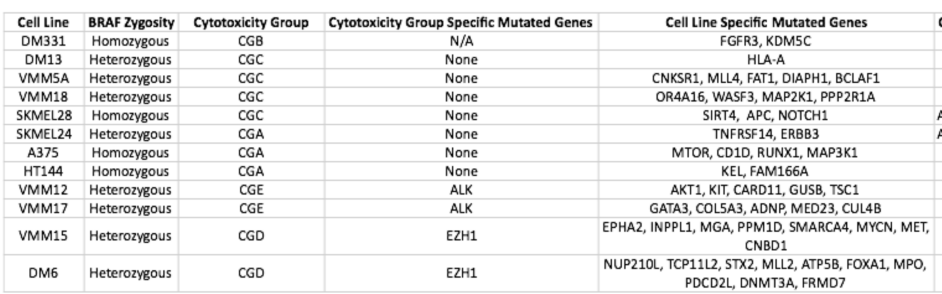

Figure 2.8 Exome analysis reveals trend between zygosity of V600E allele in the BRAF locus and sensitivity to PLX4720.

Ranked IC50 values for BRAFV60E melanomas from our panel and the CCLE. For lines in our panel that were also in the Cancer Cell Line Encyclopedia (Barretina et al., 2012) (CCLE), we used the IC50 values from the CCLE. Lines from our panel are denoted with "*”. Zygosity at the BRAF locus was assayed using IGV visualization of BAM files. Lines found to be homozygous for the V600E allele were colored blue, lines found to be heterozygous for the V600E allele were colored red, and lines found to be wild type at the BRAF locus were colored green (A). Box plots of IC50 values for 
BRAFV600E/V600E (Homozygous), BRAFWT/V600E (Heterzygous), and BRAFWT/WT (Wild Type), melanoma cell lines (B). Exome sequencing identifies potential causal variants. Variants present in our lines were compiled using exome sequencing and cross referenced with genes identified as being mutated across multiple cancer types (Lawrence et al., 2014). BRAF zygosity, genes uniquely mutated in each drug cytotoxicity group, and the genes uniquely mutated in each line are listed (C).

We then searched for genes that contained variants that were unique to individual cytotoxicity groups and could be dominant drivers of drug sensitivity within each group. We found that lines in both the CGE and CGD groups contained unique genetic variants. Variants previously identified by dbSNP were removed, and we further filtered the list by identifying novel nonsynonymous variants; nonsense and missense variants only. The list of 10,000 variants was narrowed to 143 variants across 83 genes that were identified as being known drivers of cancer (Lawrence et al., 2014). We imported the list of variants into polyphen2 (Adzhubei et al., 2010), which identifies variants that may alter function. We identified cytotoxicity group specific mutations in ALK and EZH1 that were predicted to be damaging by polyphen2 in CGD and CGE cell lines, respectively. ALK is a receptor tyrosine kinase that has been previously associated with multiple cancer types (Lawrence et al., 2014; Soda et al., 2007; Wagle et al., 2011) and is an activating mutation in many types of lung cancer (Wagle et al., 2011). EZH1 is a histone methyltransferase that deposits methyl groups on histone H3 lysine 27 (H3K27me3), which tends to yield repressed chromatin (Shen et al., 2008). EZH1 has been shown to maintain stem cell identity and pluripotency (Shen et al., 2008). Importantly, EZH1 and EZH2 have been identified as candidate oncogenes in diffuse large B cell lymphoma (Garapaty-Rao et al., 2013). 
While we found no cytotoxicity group specific variants in CGA and CGC cell lines, BRAF zygosity potentially explains the response of three of the four CGC and two of the three CGA cell lines to PLX4720 treatment respectively. Consequently, we searched for genes that contained variants specifically in the cell lines that were cytotoxicity group exceptions with regard to BRAF zygosity (SKMEL28 and SKMEL24) that were not shared by other group members. We also identified variants that were specific to SKMEL28 and SKMEL24, which could explain their being placed in CGC and CGA groups, respectively.

In SKMEL28, we found several genes that could explain its increased resistance to PLX4720 treatment compared to other homozygous BRAF lines. SKMEL28, uniquely in our panel, contains a mutation in NOTCH1, a transmembrane protein that has been shown to interact with membrane bound ligands (Rebay et al., 1991) and it can also activate MAPK signaling and PI3K signaling in melanoma (Liu et al., 2006). SKMEL28 also contains notable variants in three tumor suppressors. ARID1A and PTEN are mutated in SKMEL28 but not in its cytotoxicity group members. However, ARID1A is also mutated in VMM15, and PTEN is not mutated in any other line. ARID1A is a SWI/SNF family member and potential tumor suppressor in melanoma (Oike et al., 2013). Lastly, SKMEL28 is the only line in our panel with a mutation in APC, a tumor suppressor that antagonizes WNT signaling (Lawrence et al., 2014) and has been shown to be nonfunctional in patients with melanoma (Rubinfeld et al., 1997). We hypothesize that this set of mutations in growth pathways and tumor suppressors potentially explains SKMEL28's resistance to PLX4720 treatment, despite its homozygous BRAF status. 
In SKMEL24 we found a number of cell surface proteins that contain mutations including TNFRSF14, DNER, ERBB3 and EGFR (Figure 2.8C). A loss of function mutation in any of these proteins could limit SKMEL24 in its ability to activate alternative growth pathways in the presence of BRaf inhibition, thereby, potentially explaining its sensitivity to PLX4720 treatment despite its heterozygous mutant BRAF status. When considering its set of cell line-specific variants, the ERBB3 mutation is notable given that ERBB family members are lapatinib targets. However, polyphen2 predicted this variant to be benign, with the $1077^{\text {th }}$ residue substituting an arginine for a tryptophan, so more analyses are required to determine the significance of this variant.

Finally, we applied our search for genes that contained variants in only one cell line in order to identify variants that could be driving cell line specific cytotoxicity responses. DM331 is our most aberrant line in that it contains homozygous BRAFV600E, yet it is by far the most PLX4720 resistant cell line. We found that FGFR3, which is known to activate Ras (Teven et al., 2014), had a potential function altering mutation in the kinase domain as identified by polyphen2 (i.e., substituting an arginine (R) for a lysine (K) at residue 618). We also previously observed its high expression of NRG1, a known contributor to PLX4720 resistance (Zhang et al., 2013) and find that its basal expression of EGFR is far higher than is seen in the other lines (Roller et al., 2016). Taken together, we find evidence for the hypothesis that the sensitivity of the lines to PLX4720 treatment can be explained in part by a combination of zygosity at the BRAF locus and the mutational status of various cell surface receptors. 


\subsubsection{Cytotoxic Group-Wise Analysis After PLX4720 Treatment Reveals Broad ERBB Pathway Gene Up-regulation in Combination Sensitive Cell Lines Only}

Because basal gene expression analysis revealed modest associations with

drug sensitivity, we determined whether PLX4720 sensitivity and synergy with lapatinib could be due to changes in gene expression induced by PLX4720 treatment. To test this hypothesis, we generated and analyzed transcriptional and proteomic profiles for each of the cell lines 8 hours after PLX4720 treatment. Given the robust grouping of the cell lines according to cytotoxic drug response, we performed a group-wise analysis in order to gain power to detect subtle but consistent changes in gene expression in response to PLX4720. Specifically, we used a paired t-test within cytotoxicity groups on the transcriptional response to PLX4720 treatment matching each treatment sample with a control (i.e., no drug treatment) and applying a stringent 1\% FDR cutoff. The CGA, CGC, CGD and CGE groups (Figure 2.6A) showed 2614, 7310, 2383, and 2993 probes detecting differential gene expression at a $1 \%$ FDR, respectively. When each of these gene lists was put through a Pathway Express (Draghici et al., 2003; Khatri et al., 2002) enrichment analysis, the top two pathways in all four groups were always leukocyte transendothelial migration and cell adhesion molecules, and the third ranked pathway in every group except CGC was adherens junctions (Table 2.3). Only CGC the group requiring both lapatinib and PLX4720 for maximal cytotoxicity -- had upregulation of the noncanonical WNT and MAPK signaling pathways, which was not observed in the other groups based on a Bonferroni corrected gamma p-value of 0.01. Notably,CGC and CGA have significant Bonferroni corrected p-values for the 
ErbB signaling pathway (5.60e-05 and 4.86e-04 respectively). Visualization of differentially expressed genes in the ErbB signaling pathway across cytotoxicity groups gave the most insight into a potential mechanism for synergy and resistance (Figure 2.6B, Figure 2.9). In CGC, there is broad upregulation (red) of the ErbB signaling pathway. Only in the CGC group do we observe the upregulation of multiple ERBB family members. In CGA and CGD, only ERBB2 is upregulated, and in CGE the ERBB family is not significantly affected by treatment at all. Consistent with this result, Abel et al. have previously observed ERBB3 activation in response to vemurafenib treatment (Abel et al., 2013). When we looked at the pathways in cancer diagrams (Figure 2.10), we observed that in addition to the differences in ErbB signaling pathway, WNT signaling (Figure 2.11), and MAPK signaling (Figure 2.12) were broadly affected in CGC only. In addition to the enrichment in ErbB signaling in CGA, we also observe significant enrichments in apoptosis, a number of cancer specific pathways, and p53 signaling pathways.

Table 2.3 Pathway express analysis results for differentially expressed genes in response to PLX4720 treatment for all cytotoxicity groups.

\begin{tabular}{|c|c|c|c|}
\hline Rank & Pathway Name & Corrected gamma p-value & Cytotoxicity Group \\
\hline 1 & $\begin{array}{l}\text { Cell adhesion molecules } \\
\text { (CAMs) }\end{array}$ & $1.01 \mathrm{E}-20$ & CGA \\
\hline 2 & $\begin{array}{l}\text { Leukocyte transendothelial } \\
\text { migration }\end{array}$ & $5.42 \mathrm{E}-09$ & CGA \\
\hline 3 & Adherens junction & $8.72 \mathrm{E}-09$ & CGA \\
\hline 4 & Pathways in cancer & $1.22 \mathrm{E}-07$ & CGA \\
\hline 5 & $\begin{array}{l}\text { Antigen processing and } \\
\text { presentation }\end{array}$ & $1.54 \mathrm{E}-07$ & CGA \\
\hline 6 & Small cell lung cancer & $5.22 \mathrm{E}-06$ & CGA \\
\hline 7 & Circadian rhythm & $1.78 \mathrm{E}-05$ & CGA \\
\hline 8 & $\begin{array}{l}\text { Prostate cancer } \\
\end{array}$ & $2.95 \mathrm{E}-05$ & CGA \\
\hline 9 & Focal adhesion & $1.33 \mathrm{E}-04$ & CGA \\
\hline 10 & Wnt signaling pathway & $3.63 \mathrm{E}-04$ & CGA \\
\hline 11 & ErbB signaling pathway & $4.86 \mathrm{E}-04$ & CGA \\
\hline 12 & Colorectal cancer & $5.39 \mathrm{E}-04$ & CGA \\
\hline 13 & Non-small cell lung cancer & $7.30 \mathrm{E}-04$ & CGA \\
\hline 14 & $\begin{array}{c}\text { Adipocytokine signaling } \\
\text { pathway } \\
\end{array}$ & $7.81 \mathrm{E}-04$ & CGA \\
\hline 15 & Apoptosis & 0.001349037 & CGA \\
\hline
\end{tabular}




\begin{tabular}{|c|c|c|c|}
\hline 16 & Axon guidance & 0.0019749 & CGA \\
\hline 17 & Basal cell carcinoma & 0.004128721 & CGA \\
\hline 18 & Glioma & 0.004281121 & CGA \\
\hline 19 & Pancreatic cancer & 0.004878647 & CGA \\
\hline 20 & p53 signaling pathway & 0.006613412 & CGA \\
\hline 21 & Jak-STAT signaling pathway & 0.007037892 & CGA \\
\hline 22 & TGF-beta signaling pathway & 0.00732704 & CGA \\
\hline 23 & MAPK signaling pathway & 0.008094704 & CGA \\
\hline 24 & $\begin{array}{l}\text { Phosphatidylinositol signaling } \\
\text { system }\end{array}$ & 0.00895652 & CGA \\
\hline 1 & $\begin{array}{l}\text { Leukocyte transendothelial } \\
\text { migration }\end{array}$ & $9.24 \mathrm{E}-84$ & CGC \\
\hline 2 & $\begin{array}{c}\text { Cell adhesion molecules } \\
\text { (CAMs) }\end{array}$ & $1.44 \mathrm{E}-57$ & CGC \\
\hline 3 & $\begin{array}{c}\text { Cytokine-cytokine receptor } \\
\text { interaction }\end{array}$ & $2.85 \mathrm{E}-09$ & CGC \\
\hline 4 & Pathways in cancer & $7.25 \mathrm{E}-08$ & CGC \\
\hline 5 & $\begin{array}{l}\text { Neuroactive ligand-receptor } \\
\text { interaction }\end{array}$ & $2.46 \mathrm{E}-07$ & CGC \\
\hline 6 & Focal adhesion & $3.65 \mathrm{E}-07$ & CGC \\
\hline 7 & MAPK signaling pathway & $1.12 \mathrm{E}-06$ & CGC \\
\hline 8 & Jak-STAT signaling pathway & $1.43 \mathrm{E}-06$ & CGC \\
\hline 9 & Melanoma & $6.58 \mathrm{E}-06$ & CGC \\
\hline 10 & $\begin{array}{l}\text { Phosphatidylinositol signaling } \\
\text { system }\end{array}$ & $6.74 \mathrm{E}-06$ & CGC \\
\hline 11 & ErbB signaling pathway & $5.60 \mathrm{E}-05$ & CGC \\
\hline 12 & Wnt signaling pathway & $5.96 \mathrm{E}-05$ & CGC \\
\hline 13 & Adherens junction & $1.29 \mathrm{E}-04$ & CGC \\
\hline 14 & $\begin{array}{l}\text { Regulation of actin } \\
\text { cytoskeleton }\end{array}$ & $1.47 \mathrm{E}-04$ & CGC \\
\hline 15 & Axon guidance & 1.77E-04 & CGC \\
\hline 16 & Glioma & $2.54 \mathrm{E}-04$ & CGC \\
\hline 17 & Tight junction & $2.59 \mathrm{E}-04$ & CGC \\
\hline 18 & $\begin{array}{c}\text { T cell receptor signaling } \\
\text { pathway }\end{array}$ & $2.65 \mathrm{E}-04$ & CGC \\
\hline 19 & Calcium signaling pathway & $3.60 \mathrm{E}-04$ & CGC \\
\hline 20 & PPAR signaling pathway & $6.17 \mathrm{E}-04$ & CGC \\
\hline 21 & Olfactory transduction & $7.30 \mathrm{E}-04$ & CGC \\
\hline 22 & Non-small cell lung cancer & $8.10 \mathrm{E}-04$ & CGC \\
\hline 23 & $\begin{array}{l}\text { Antigen processing and } \\
\text { presentation }\end{array}$ & 8.37E-04 & CGC \\
\hline 24 & $\begin{array}{c}\text { Complement and coagulation } \\
\text { cascades }\end{array}$ & 0.001371042 & CGC \\
\hline 25 & $\begin{array}{l}\text { Maturity onset diabetes of the } \\
\text { young }\end{array}$ & 0.001557527 & CGC \\
\hline 26 & Primary immunodeficiency & 0.001938145 & $\overline{C G C}$ \\
\hline 27 & $\begin{array}{c}\text { Natural killer cell mediated } \\
\text { cytotoxicity }\end{array}$ & 0.002260202 & CGC \\
\hline 28 & Prostate cancer & 0.002824351 & CGC \\
\hline 29 & mTOR signaling pathway & 0.003802526 & CGC \\
\hline 30 & TGF-beta signaling pathway & 0.004180151 & CGC \\
\hline 31 & Chronic myeloid leukemia & 0.005022655 & CGC \\
\hline 32 & Hematopoietic cell lineage & 0.005931458 & CGC \\
\hline 33 & Bladder cancer & 0.005999586 & CGC \\
\hline 34 & p53 signaling pathway & 0.006335204 & $\overline{C G C}$ \\
\hline 35 & Renal cell carcinoma & 0.006659957 & CGC \\
\hline 36 & Thyroid cancer & 0.007954554 & CGC \\
\hline 37 & Acute myeloid leukemia & 0.008338527 & CGC \\
\hline 1 & $\begin{array}{c}\text { Leukocyte transendothelial } \\
\text { migration }\end{array}$ & $9.04 \mathrm{E}-133$ & GDC \\
\hline 2 & Cell adhesion molecules & $7.63 E-55$ & GDC \\
\hline
\end{tabular}




\begin{tabular}{|c|c|c|c|}
\hline & (CAMs) & & \\
\hline 3 & Adherens junction & $1.33 \mathrm{E}-16$ & GDC \\
\hline 4 & $\begin{array}{l}\text { Phosphatidylinositol signaling } \\
\text { system }\end{array}$ & $1.99 \mathrm{E}-11$ & GDC \\
\hline 5 & Parkinson"s disease & $2.27 \mathrm{E}-07$ & GDC \\
\hline 6 & Circadian rhythm & $5.90 \mathrm{E}-07$ & GDC \\
\hline 7 & $\begin{array}{l}\text { Antigen processing and } \\
\text { presentation }\end{array}$ & $7.82 \mathrm{E}-07$ & GDC \\
\hline 8 & Alzheimer"s disease & $2.74 \mathrm{E}-06$ & GDC \\
\hline 9 & Pathways in cancer & $1.07 \mathrm{E}-05$ & GDC \\
\hline 10 & Huntington"s disease & $6.59 \mathrm{E}-05$ & GDC \\
\hline 11 & p53 signaling pathway & 0.001885117 & GDC \\
\hline 12 & Bladder cancer & 0.004284906 & GDC \\
\hline 13 & Wnt signaling pathway & 0.005947112 & GDC \\
\hline 14 & Pancreatic cancer & 0.007062585 & GDC \\
\hline 15 & Colorectal cancer & 0.008612032 & GDC \\
\hline 1 & $\begin{array}{l}\text { Cell adhesion molecules } \\
\text { (CAMs) }\end{array}$ & $2.23 E-49$ & CGE \\
\hline 2 & $\begin{array}{c}\text { Leukocyte transendothelial } \\
\text { migration }\end{array}$ & $2.65 E-47$ & CGE \\
\hline 3 & Adherens junction & $4.93 \mathrm{E}-10$ & CGE \\
\hline 4 & Circadian rhythm & $2.78 \mathrm{E}-06$ & CGE \\
\hline 5 & Pathways in cancer & $8.94 \mathrm{E}-06$ & CGE \\
\hline 6 & Small cell lung cancer & $3.51 \mathrm{E}-05$ & CGE \\
\hline 7 & Prostate cancer & $1.26 \mathrm{E}-04$ & CGE \\
\hline 8 & Colorectal cancer & $7.32 \mathrm{E}-04$ & CGE \\
\hline 9 & Renal cell carcinoma & 8.13E-04 & CGE \\
\hline 10 & $\begin{array}{l}\text { Ubiquitin mediated } \\
\text { proteolysis }\end{array}$ & 8.25E-04 & CGE \\
\hline 11 & Bladder cancer & 0.001292046 & CGE \\
\hline 12 & Endometrial cancer & 0.001582886 & CGE \\
\hline 13 & Thyroid cancer & 0.00177535 & CGE \\
\hline 14 & Wnt signaling pathway & 0.00250439 & CGE \\
\hline 15 & Basal cell carcinoma & 0.002784422 & CGE \\
\hline 16 & $\begin{array}{l}\text { Epithelial cell signaling in } \\
\text { Helicobacter pylori infection }\end{array}$ & 0.002814316 & CGE \\
\hline 17 & Focal adhesion & 0.005403562 & CGE \\
\hline 18 & \$hsa05131\$ & 0.005926249 & CGE \\
\hline 19 & $\begin{array}{c}\text { Pathogenic Escherichia coli } \\
\text { infection }\end{array}$ & 0.005926249 & CGE \\
\hline 20 & RNA polymerase & 0.006885467 & CGE \\
\hline 21 & Glioma & 0.008172814 & CGE \\
\hline 22 & Pancreatic cancer & 0.0093714 & CGE \\
\hline 23 & TGF-beta signaling pathway & 0.0093714 & CGE \\
\hline
\end{tabular}




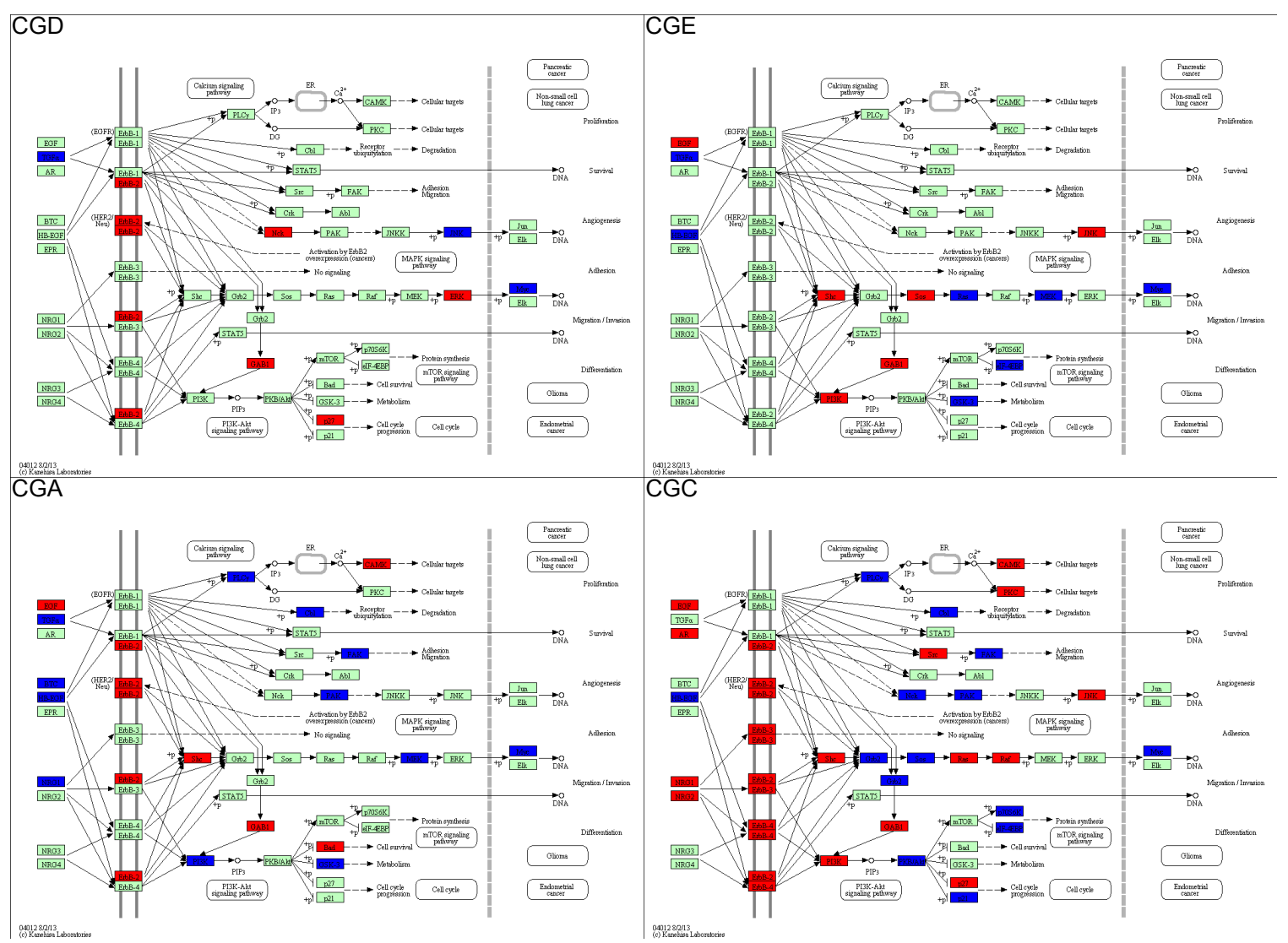

Figure 2.9 ErbB signaling pathway response to PLX4720 treatment for each cytotoxicity group.

The responses for each group are CGD (top left), CGE (top right), CGA (bottom left), and CGC (bottom right). Nodes in red denote an upregulation, nodes in blue denote a downregulation. 

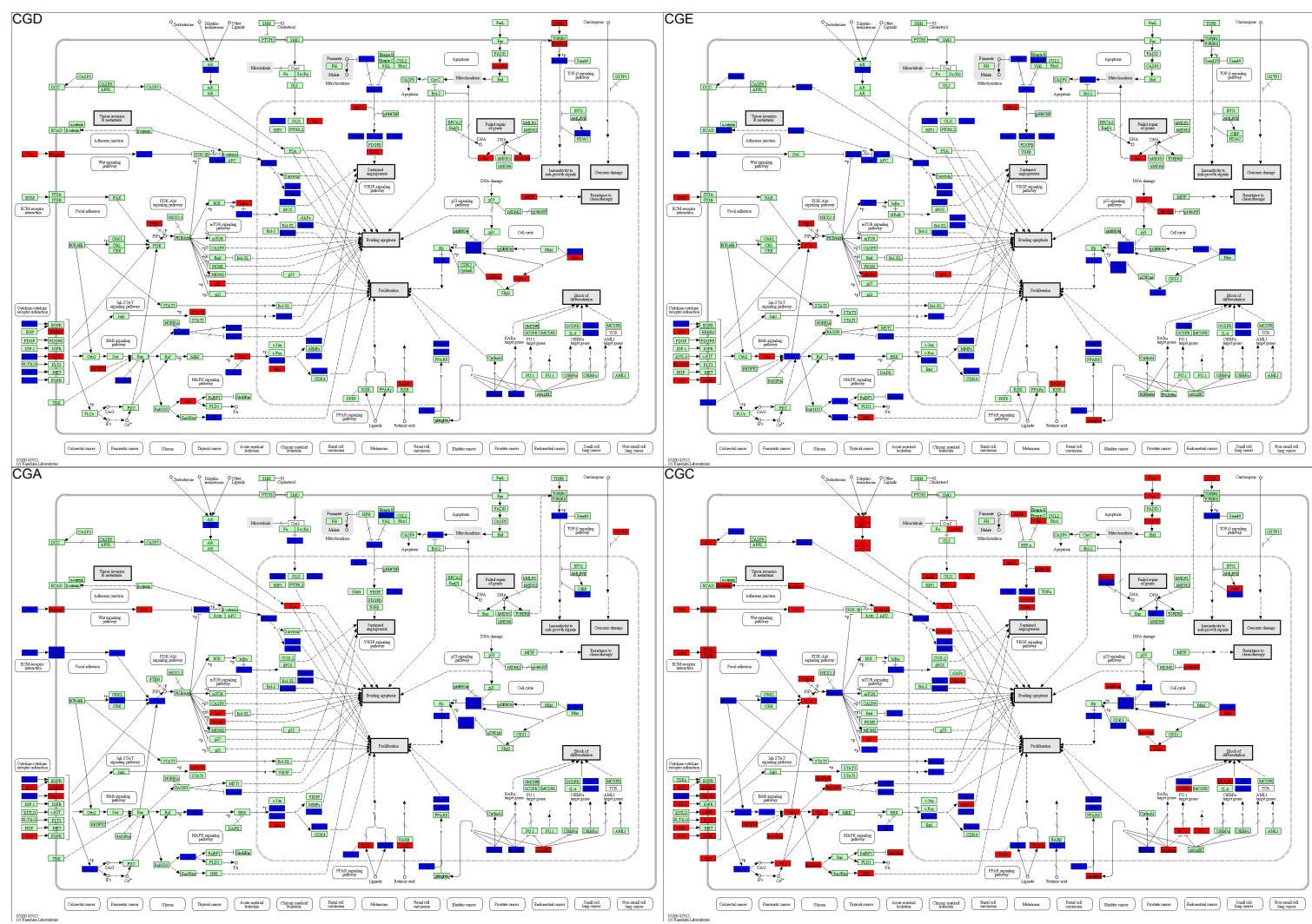

Figure 2.10 Pathways in cancer pathway response to PLX4720 treatment for each cytotoxicity group.

The responses for each group are CGD (top left), CGE (top right), CGA (bottom left), and CGC (bottom right). Nodes in red denote an upregulation, nodes in blue denote a downregulation. 


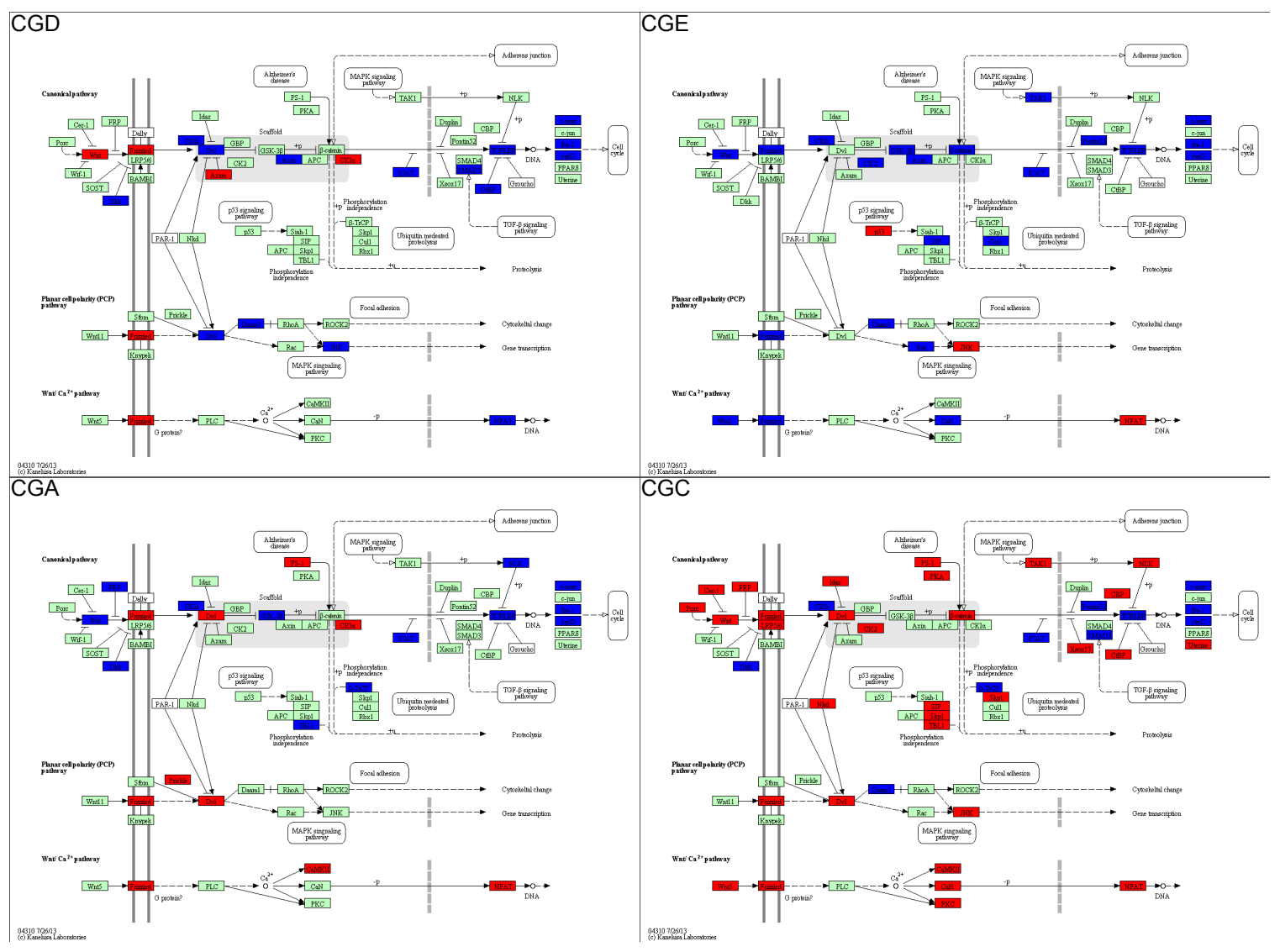

Figure 2.11 Wnt signaling pathway response to PLX4720 treatment for each cytotoxicity group.

The responses for each group are CGD (top left), CGE (top right), CGA (bottom left), and CGC (bottom right). Nodes in red denote an upregulation, nodes in blue denote a downregulation. 


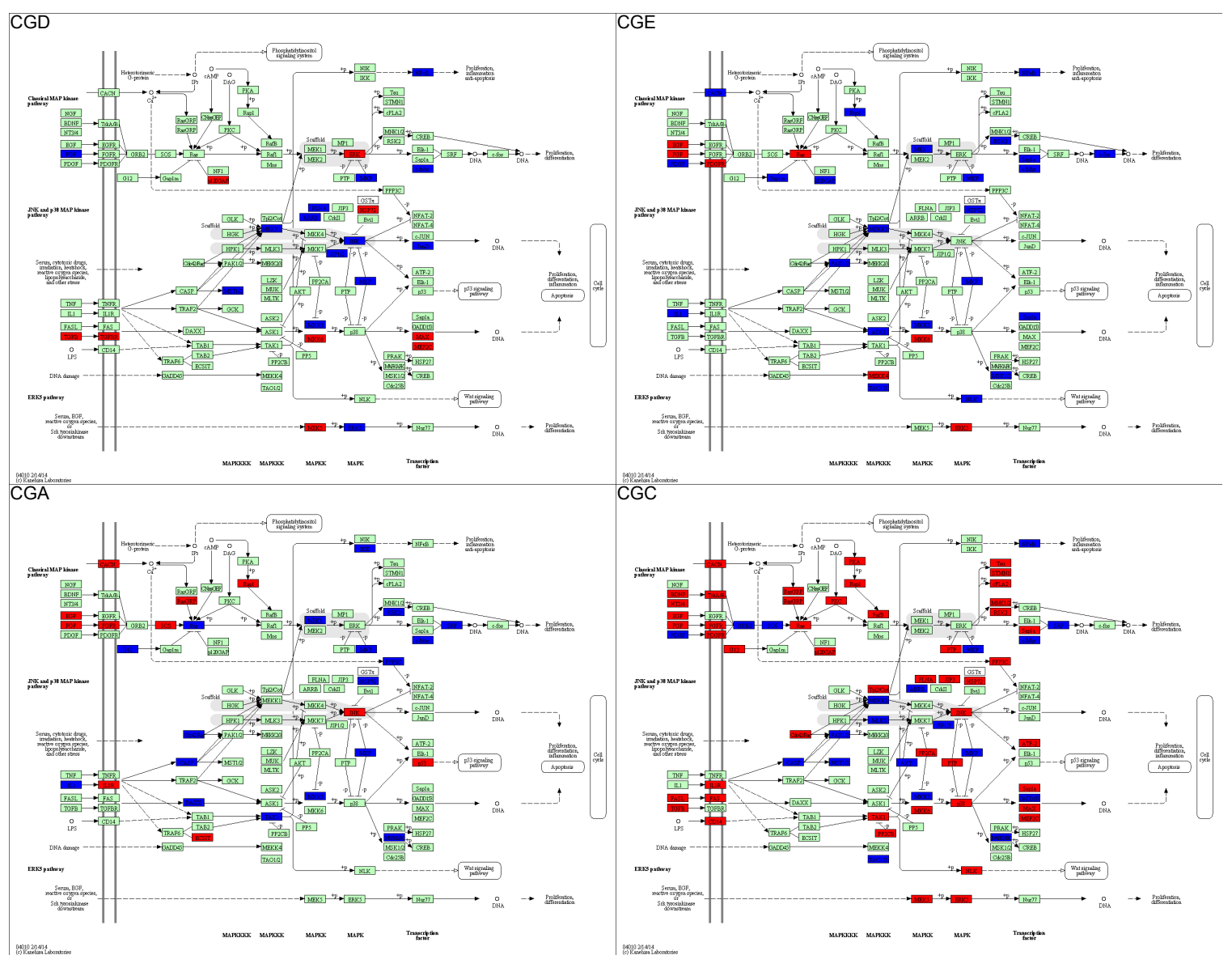

Figure 2.12 MAPK signaling pathway response to PLX4720 treatment for each cytotoxicity group.

The responses for each group are CGD (top left), CGE (top right), CGA (bottom left), and CGC (bottom right). Nodes in red denote an upregulation, nodes in blue denote a downregulation. 
Given the striking, simple result that the ERBB pathway is broadly upregulated in response to PLX4720 treatment only in the group of cell lines that require treatment with the PLX4720 and lapatinib combination in order to achieve an optimally cytotoxic response, we asked which transcription factors were likely activated by mutant BRaf inhibition and targeting ERBB pathway genes. Using MSigDB (Subramanian et al., 2005), we identified transcription factors whose motif occurrence is significantly enriched at the promoters of genes that were differentially expressed in each cytotoxicity group (Table 2.4). We found 45 unique transcription factor binding motifs that are only present in CGC, among which were an ETS family member and its associated cofactors, including ETS2, GATA1, and POU2F1. Additionally, we saw a number of well-established oncogenic transcription factor binding motifs, including Jun and Myb. Lastly, based on ChIP-seq data (Strub et al., 2011), we found that MITF targets a number of ERBB pathway genes (Ji et al., 2015).

Table 2.4 MSigDB transcription factor enrichment results for differentially expressed genes in response to PLX4720 for all cytotoxicity groups

\begin{tabular}{|c|c|c|c|c|}
\hline Gene Set Name & Description & FDR q-value & Rank & $\begin{array}{c}\text { Cytotoxicity } \\
\text { Group }\end{array}$ \\
\hline CAGGTG_V\$E12_Q6 & Motif CAGGTG; Gene TCF3 & $2.20 E-79$ & 1 & CGC \\
\hline TTGTTT_V\$FOX04_01 & Motif TTGTT; Gene MLLT7 & $2.86 E-74$ & 2 & CGC \\
\hline GGGAGGR_V\$MAZ_Q6 & Motif GGGAGGRR; Gene MAZ & $4.60 E-69$ & 3 & CGC \\
\hline AACTTT_UNKNOWN & Motif AACTTT; Gene unknown & $9.57 E-68$ & 4 & CGC \\
\hline TGGAAA_V\$NFAT_Q4_01 & Motif TGGAAA; Gene NFAT & $1.67 E-67$ & 5 & CGC \\
\hline GGGCGGR_V\$SP1_Q6 & Motif GGGCGGR; Gene SP1 & $1.03 E-58$ & 6 & CGC \\
\hline CTTTGT_V\$LEF1_Q2 & Motif CTTTGT; Gene LEF1 & $1.36 E-56$ & 7 & CGC \\
\hline TATAAA_V\$TATA_01 & Motif TATAAA; Gene TAF & $3.64 E-52$ & 8 & CGC \\
\hline CAGCTG_V\$AP4_Q5 & Motif CAGCTG; Gene REPIN1 & $4.14 E-43$ & 9 & CGC \\
\hline CTTTGA_V\$LEF1_Q2 & Motif CTTTGA; Gene LEF1 & $7.71 E-42$ & 10 & CGC \\
\hline GGGCGGR_V\$SP1_Q6 & Motif GGGCGGR; Gene SP1 & $4.10 E-118$ & 1 & CGA \\
\hline GGGAGGRR_V\$MAZ_Q6 & Motif GGGAGGRR; Gene MAZ & $3.78 E-91$ & 2 & CGA \\
\hline CTTTGT_V\$\$LF1_Q2 & Motif CTTTGT; Gene LEF1 & $1.92 E-81$ & 3 & CGA \\
\hline CAGGTG_V\$E12_Q6 & Motif CAGGTG; Gene TCF3 & $5.37 E-78$ & 4 & CGA \\
\hline TTGTT_V\$FOX04_01 & Motif TTGTTT; Gene MLLT7 & $6.34 E-72$ & 5 & CGA \\
\hline AACTTT_UNKNOWN & Motif AACTT; Gene unknown & $7.16 E-71$ & 6 & CGA \\
\hline TGGAAA_V\$NFAT_Q4_01 & Motif TGGAAA; Gene NFAT & $3.23 E-61$ & 7 & CGA \\
\hline CACGTG_V\$MYC_Q2 & Motif CACGTG; Gene MYC & $6.83 E-55$ & 8 & CGA \\
\hline
\end{tabular}




\begin{tabular}{|c|c|c|c|c|}
\hline GATTGGY_V\$NFY_Q6_01 & Motif GATTGGY; Gene unknown & $1.43 E-54$ & 9 & CGA \\
\hline CAGCTG_V\$AP4_Q5 & Motif CAGCTG; Gene REPIN1 & $1.86 E-52$ & 10 & CGA \\
\hline GGGCGGR_V\$SP1_Q6 & Motif GGGCGGR; Gene SP1 & $2.02 E-111$ & 1 & CGE \\
\hline SCGGAAGY_V\$ELK1_02 & Motif SCGGAAGY; Gene ELK1 & $1.58 E-75$ & 2 & CGE \\
\hline GGGAGGR_V\$MAZ_Q6 & Motif GGGAGGRR; Gene MAZ & $1.41 E-74$ & 3 & CGE \\
\hline CAGGTG_V\$E12_Q6 & Motif CAGGTG; Gene TCF3 & $1.09 E-71$ & 4 & CGE \\
\hline CTTTGT_V\$LEF1_Q2 & Motif CTTTGT; Gene LEF1 & $1.10 E-70$ & 5 & CGE \\
\hline TTGTTT_V\$FOX04_01 & Motif TTGTTT; Gene MLLT7 & $5.37 E-66$ & 6 & CGE \\
\hline AACTTT_UNKNOWN & Motif AACTTT; Gene unknown & $1.58 E-59$ & 7 & CGE \\
\hline TGGAAA_V\$NFAT_Q4_01 & Motif TGGAAA; Gene NFAT & $1.59 E-55$ & 8 & CGE \\
\hline CACGTG_V\$MYC_Q2 & Motif CACGTG; Gene MYC & $4.92 E-53$ & 9 & CGE \\
\hline GATTGGY_V\$NFY_Q6_01 & Motif GATTGGY; Gene unknown & $2.18 E-44$ & 10 & CGE \\
\hline GGGCGGR_V\$SP1_Q6 & Motif GGGCGGR; Gene SP1 & $1.43 E-98$ & 1 & CGD \\
\hline CTTTGT_V\$LEF1_Q2 & Motif CTTTGT; Gene LEF1 & $2.27 E-79$ & 2 & CGD \\
\hline TTGTTT_V\$FOX04_01 & Motif TTGTT; Gene MLLT7 & $1.34 E-73$ & 3 & CGD \\
\hline AACTTT_UNKNOWN & Motif AACTTT; Gene unknown & $6.03 E-63$ & 4 & CGD \\
\hline GGGAGGRR_V\$MAZ_Q6 & Motif GGGAGGRR; Gene MAZ & $8.44 E-59$ & 5 & CGD \\
\hline TGGAAA_V\$NFAT_Q4_01 & Motif TGGAAA; Gene NFAT & $3.34 E-54$ & 6 & CGD \\
\hline CAGGTG_V\$E12_Q6 & Motif CAGGTG; Gene TCF3 & $3.16 E-51$ & 7 & CGD \\
\hline SCGGAAGY_V\$ELK1_02 & Motif SCGGAAGY; Gene ELK1 & $4.46 E-44$ & 8 & CGD \\
\hline CACGTG_V\$MYC_Q2 & Motif CACGTG; Gene MYC & $1.28 E-42$ & 9 & CGD \\
\hline TGANTCA_V\$AP1_C & Motif TGANTCA; Gene JUN & $1.41 E-41$ & 10 & CGD \\
\hline
\end{tabular}

We further analyzed transcription factor motifs that appear across multiple

cytotoxicity groups in order to identify those that were more strongly enriched in

CGC. For motifs that appeared in more than one group, we added the motif's rank in

CGC to the motif's rank in the other group subtracted from 100. For motifs that

appeared in more than the CGC and one other group, we calculated the arithmetic

mean of the 100 minus the other groups' ranks, and added that to the CGC rank.

Using these averaged ranks summed with the rank for CGC, we found that CGC is

more enriched than any other group for an additional 28 transcription factor

binding motifs. We then found which of these transcription factors were associated

with the promoters of ErbB signaling pathway genes. To visualize the ERBB

pathway gene responses to PLX4720 treatment together with the transcription

factors that putatively regulate them in one view, we plotted the log 2 fold change of

PLX4720 treated versus control of genes in the ErbB signaling pathway for each

cytotoxicity group (Figure 2.13 and Figure 2.14, Left) and identified transcription

factors that have predicted binding motifs for those genes (Figure 2.13 and Figure 
2.14, Right). There is a subset of these factors closest to the heat map including LEF1, SP1, AP4, ETS2, and PAX4 that putatively target multiple upregulated genes in the largest central cluster of the heat map. Additionally, ERBB family members contain binding motifs for LEF1, SP1, AP4, NFAT, MAZ, and FOXO4 at their promoters. Notably, many of these factors have been associated with melanoma. LEF1 has been shown to be highly expressed in proliferative cells in melanoma (Eichhoff et al., 2011; Xu et al., 2014). In a HMGB1 knockdown in melanoma, a marked decrease in cell proliferation was observed mediated by p21 in a SP1 dependent manner (Li et al., 2014). NFAT has been identified as a potential drug target in melanoma using aminobisphosphonate zoledronic acid (Levin-Gromiko et al., 2014). Lastly, FOXO4 activation can induce senescence in BRAFV600E mutants (Keizer et al., 2010). Putting these results together, we find that transcription factors associated with MAPK signaling including ETS family members and cofactors along with factors that contain MAPK consensus sites (Diella et al., 2008) are apparently activated in response to PLX4720 treatment in combination sensitive cell lines to upregulate ERBB pathway genes, priming the cells for sensitivity to lapatinib. 


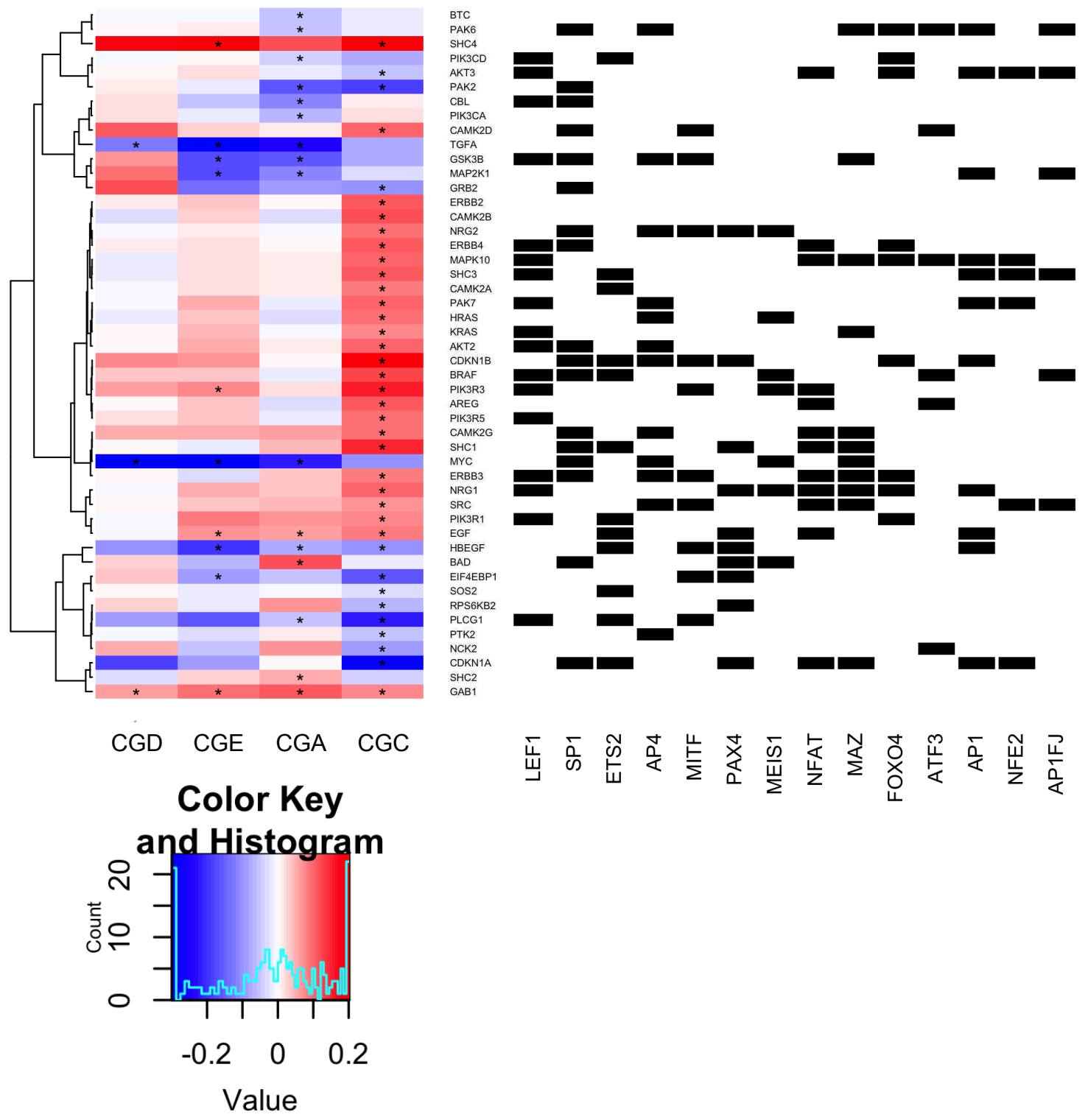

Figure 2.13 Transcription factors associated with ERBB pathway upregulated in combination sensitive cell lines treated with PLX4720.

Unsupervised clustering of gene expression response ( $\log _{2}$ fold change) to PLX4720 treatment (y-axis) of the ErbB signaling pathway genes of each cytotoxicity group (left heat map). Transcription factors ( $x$-axis) that have binding motifs in the promoters of ErbB signaling pathway genes (black squares in binary matrix on right). 


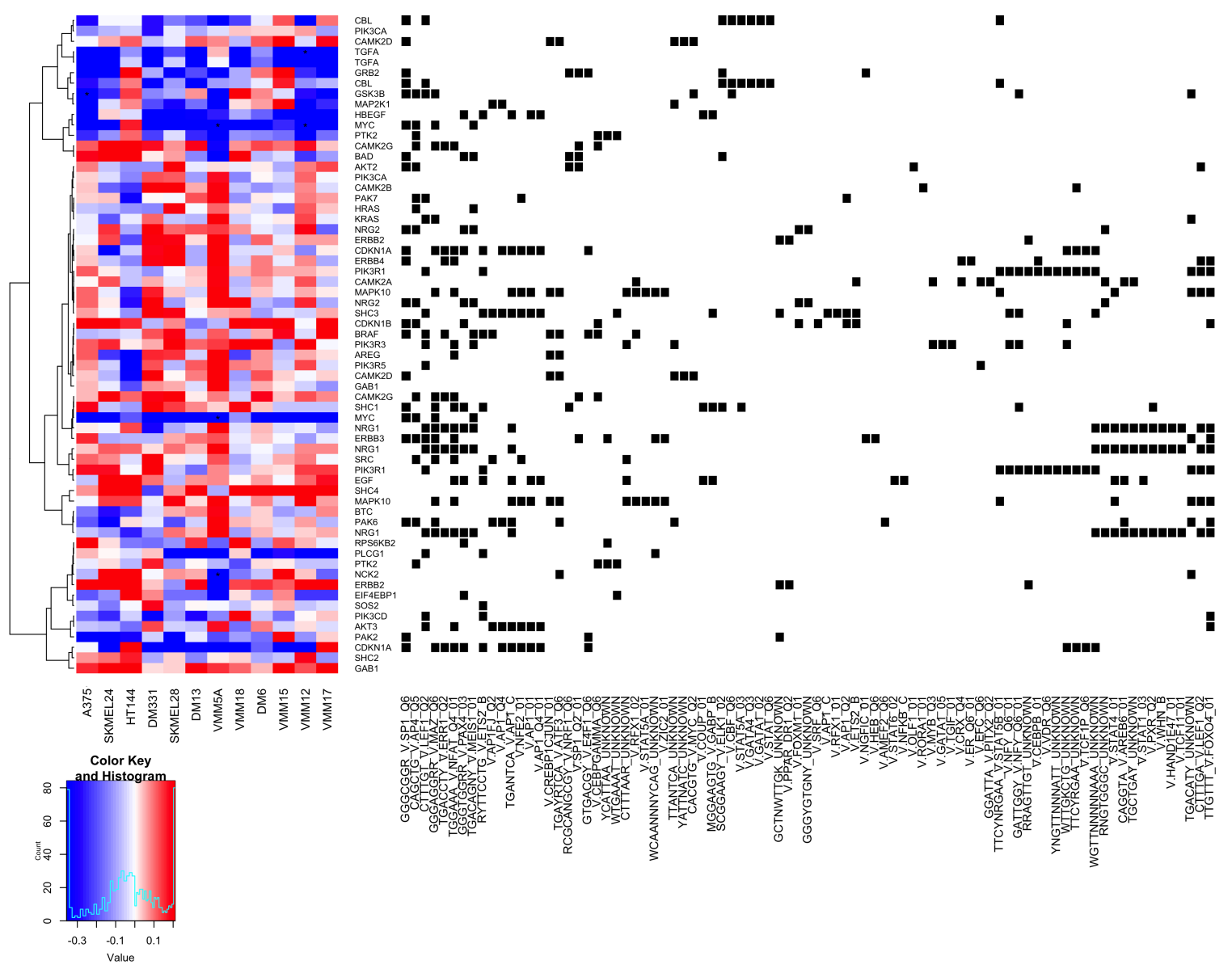

Figure 2.14 Transcription factor enrichment of ErbB signaling pathway for all lines

Unsupervised clustering of gene expression response to PLX4720 treatment (y-axis) of the ErbB signaling pathway of each cell line (Left). Transcription factors were identified that have binding motifs in the promoters of these genes (Right). 


\subsubsection{Reverse Phase Protein Array Analysis Reveals Down-regulation of Oncogenic Proteins Across Cytotoxicity Groups Eight Hours After PLX4720 Treatment}

To test our hypothesis that resistant lines upregulate ErbB signaling activity, we performed reverse phase protein array analysis. We assayed approximately 132 protein epitopes across 105 different proteins sampling various oncogenic pathways including MAPK, PI3K, ERBB, etc. eight hours after initial treatment with PLX4720. We $\log 2$ transformed the normalized values, performed cytotoxic groupwise paired t-tests as described above and applied a 5\% FDR cutoff. We identified 36 differentially expressed phosphoproteins, one cleavage site, and two proteins, whose total amount changed in response to PLX4720 treatment. Using these epitopes, we plotted their fold changes for each group due to treatment with PLX4720 in a heat map (Figure 2.15). Surprisingly, as shown in Figure 6A, the lapatinib and combination resistant (CGD) and lapatinib resistant (CGE) groups display the strongest response to PLX4720 treatment with the majority of phosphoprotein levels going down. Notably, while not statistically significant by our stringent cutoff, the phosphoproteins in the other cytotoxicity groups displayed similar but weaker trends as those in the two resistant groups. This result together with the fact that we are assaying phosphoprotein levels eight hours after initial drug treatment may indicate that we are observing the direct downstream response of BRaf inhibition with minimal compensatory signaling being manifested at the level of protein phosphorylation. Interestingly, the weaker reduction observed at the protein level in combination sensitive (CGC) and PLX4720 sensitive (CGA) cells may be due to delayed compensatory signaling, which includes transcription and 
translation (Joseph et al., 2010). This combination of a direct response of PLX4720 (i.e., downregulation) and delayed compensatory signaling may explain why we do not observe upregulation of proteins in the ERBB pathway 8 hours after PLX4720 treatment. 
A

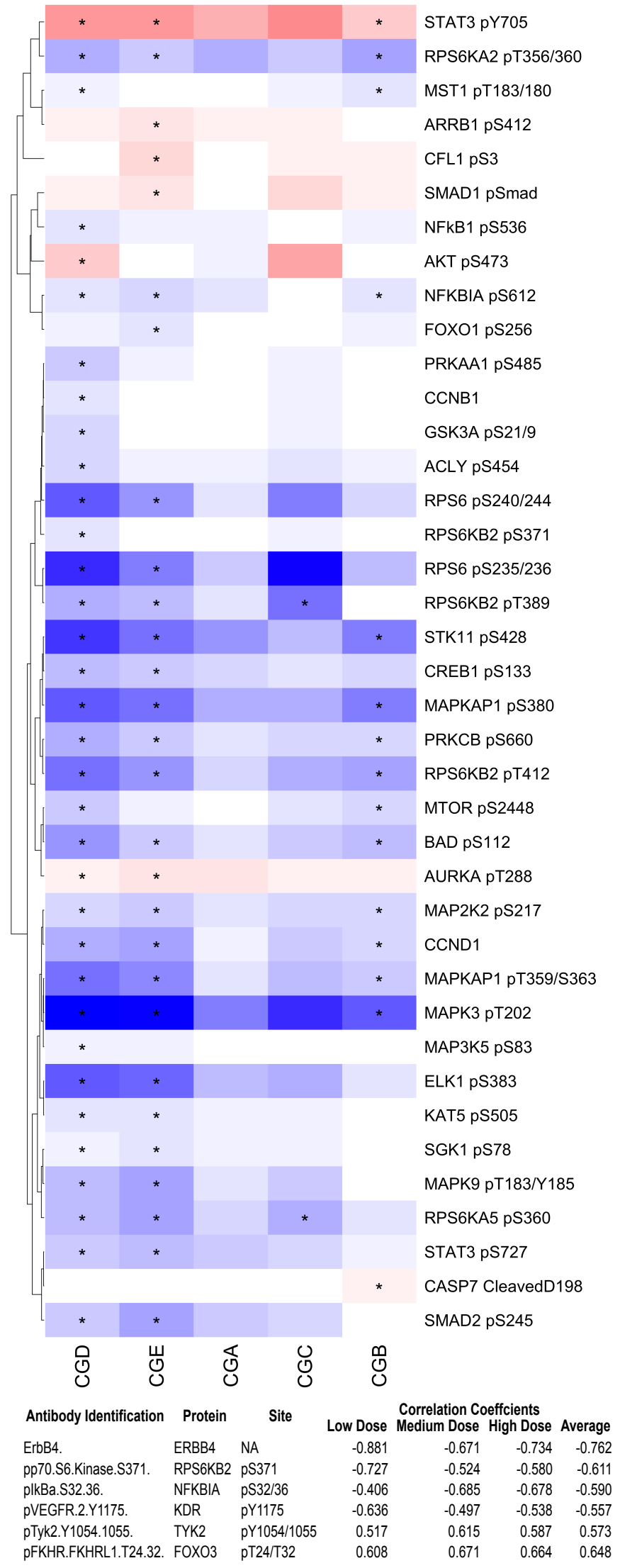




\section{Figure 2.15 Reverse phase protein array reveals stark changes to output of RTK signaling pathways}

Heat map of protein phosphorylation changes at 5\% FDR across the four cytotoxicity groups. (A). Correlating the response to treatment from the protein array with the cytotoxicity identified ERBB4 total protein fold changes as being the most anti correlated (B).

To see if there is a signature at the protein level indicating that RTKdependent signaling was being weakly but consistently downregulated in PLX4720 sensitive cell lines and upregulated in PLX4720 resistant cell lines in response to PLX4720 treatment, we calculated the Pearson correlation coefficient between the normalized log2 fold change (PLX4720 treated over untreated cells) and cytotoxicity in response to PLX4720 treatment across cell lines (including DM331) for each of the three doses separately. We found the change in total ERBB4 protein levels in response to PLX4720 treatment is the most anticorrelated epitope with PLX4720 cytotoxicity (Figure 2.15B). Other highly anticorrelated proteins were ribosomal protein S6 kinase, the alpha inhibitor of NFkB, and VEGFR. The significantly differentially phosphorylated proteins in response to PLX4720 treatment and this correlation analysis suggest that PLX4720 inhibition may be broadly activating multiple RTKs beyond ERBB family members in CGC but reducing their activity in the other groups.

\subsection{DISCUSSION}

Inhibition of BRaf signaling as a therapy for the $50 \%$ of cutaneous melanomas that are mutant BRAF ${ }^{\mathrm{V} 600 \mathrm{E}}$ is confounded by a wide variety of mechanisms of resistance, both intrinsic and acquired. Thus, there is considerable interest in understanding the general principles of resistance to BRaf inhibition so 
that druggable targets could be identified that would guide the development of effective drug combinations. In a high throughput screen, we found that the combination of the mutant BRaf inhibitor PLX4720 with the ERBB family inhibitor lapatinib caused synergistic cytotoxicity in the six lines of our 12 cell-line panel that showed the greatest intrinsic resistance to BRaf inhibition. By performing hierarchical clustering of the cytotoxicity dose-response data, we identified five different cytotoxicity groupings, reflecting extraordinary heterogeneity in biological responses to drug treatment in this panel. Molecular profiling identified lineage (reflected by MITF expression), BRAF zygosity and basal transcriptional differences as modest determinants of resistance, with cell-line specific mutations in protooncogenes and tumor suppressors and widespread transcriptional upregulation of ERBB family members and other receptor tyrosine kinases in response to PLX4720 as being the most prominent drivers of resistance. Examination of the transcriptional responses to PLX4720 treatment revealed the involvement of transcription factors with the potential to be responsive to the MAP Kinase pathway, providing a possible convergent mechanism underlying the diversity of phenotypic responses.

\subsubsection{Analysis of the basal state: cytotoxicity, transcriptome and genome.} Principal Component Analysis of the basal transcriptomes of the five cytotoxicity groups revealed a correlation between drug synergy and the second Principal Component. The strongest transcriptional correlation and anticorrelation to synergy was among genes encoding cell surface receptors including GPCRs and neuroactive ligand signaling GPCRs. Many GPCRs are capable of activating the MAP 
Kinase pathway, either directly or by transactivating RTKs utilizing neurotropin (Delcourt et al., 2007; Lee and Chao, 2001; Lee et al., 2002), thus identifying a potential mechanism for bypassing BRaf inhibition. Nevertheless, the broad and diverse genes and pathways did not yield clear, concrete mechanistic insights into drug response. The first Principal Component of the basal transcriptomic analysis correlated with MITF expression, which has been implicated by several reports in drug resistance and oncogenesis in melanoma (Hertzman Johansson et al., 2013; Søndergaard et al., 2010; Thomas and Erickson, 2009). For example, Konieczkowski et al. recently identified high MITF levels and MITF transcriptional target activity as associated with increased sensitivity to PLX4720 treatment (Konieczkowski et al., 2014). However, in our panel we obtained a very modest association between MITF and IC50 for PLX4720, an association that was driven almost entirely by our single most resistant cell line; when this line (DM331) was dropped from the analysis, no association with MITF appeared. However the study by Konieczkowski et al. (Konieczkowski et al., 2014) utilized 29 cell lines, many of which displayed lower MITF expression levels than occurred in our panel; MITF gene expression levels would be classified as MITF high for all our cell lines according to the criteria established by Muller et al. (Müller et al., 2014). Thus, the Konieczkowski study, by dint of range of MITF expression and number of cell lines, was better powered to detect a modest association.

The groupings of our cell lines according to cytotoxic response to drug treatment and basal expression profile can be rationalized in part by BRAFV600 zygosity status. The high proportion of homozygous BRAF mutants in our study (4 
out of 12) helps to highlight this tendency, and was strengthened by comparison with data in the Cell Line Encyclopedia. As shown in and Figure 2.5, cell lines (excluding SKMEL28 and SKMEL24) on the left of the PCA plot contain heterozygous $\mathrm{BRAF}^{\mathrm{V} 600 \mathrm{E}}$ mutations. Regarding cytotoxic response to vemurafenib treatment, the cell lines tend to be more resistant on the left. Additionally, the cell lines on the top of the PCA plot respond synergistically to the combination of vemurafenib and lapatinib while those on the bottom do not. It has been shown that vemurafenib binding to $\mathrm{BRAF}^{\mathrm{V} 600 \mathrm{E}}$ paradoxically activates downstream MAPK signaling via dimerization with non-mutant Raf and allosteric activation of the non-mutant partner (Holderfield et al., 2014). In a homozygous BRAFV600E cell line, opportunities for this paradoxical activation are limited to the heterodimers with CRaf, which could partially explain increased dependency on the output of the mutationally activated BRaf.

Our results support the findings of Konieczkowski et al. (Konieczkowski et al., 2014) that overexpression of BRAF ${ }^{\mathrm{V} 600 \mathrm{E}}$ suppresses MITF gene expression: in our cell lines, MITF levels tend to be lower in homozygous versus heterozygous lines. We hypothesize that BRAFV600E levels are inhibiting MITF levels, which in that sense may be acting more as a passenger. Nevertheless, MITF is a potent regulator of melanoma lineage and to a large extent determines the basal transcriptional profile, hence its association with the first Principal Component of our basal transcriptional analysis.

Layered over and interacting with $\mathrm{BRAF}^{\mathrm{6} 600 \mathrm{E}}$ zygosity and MITF levels in affecting sensitivity to PLX4720, are mutations in additional oncogenes and tumor 
suppressor genes. Mutations in cell surface proteins and RTKs potentially explain (1) cytotoxicity group-BRAF zygosity exceptions including SKMEL28 (homozygous BRAF yet combination sensitive) and SKMEL24 (heterozygous BRAF yet PLX4720 sensitive), (2) lapatinib resistant cell lines, which are heterozygous BRAF and (3) DM331, which is an outlier from a cytotoxic and transcriptional profile grouping perspective. (1) In SKMEL28, the most striking variant was the EGFR exception mutation, which has previously been identified as a gain of function mutation (Basu et al., 2013; Prahallad et al., 2012) and could explain this line's resistance to PLX4720 treatment despite being homozygous for BRAFV600E. In SKMEL24 we found a number of potentially damaging mutations in cell surface proteins including TNFRSF14, ERBB3 and EGFR, which could limit SKMEL24's ability to activate alternative growth pathways in the presence of BRaf inhibition, thereby, potentially explaining its sensitivity to PLX4720 treatment despite its heterozygous BRAF status. The ERBB3 mutation is particularly interesting given that ERBB family members are lapatinib targets; however, this mutation was not found to be damaging by polyphen 2 analysis and would require more study to determine if it alters ERBB3 function. (2) Both lapatinib resistant cell lines contained a damaging mutation in ALK, which is a receptor tyrosine kinase that has been previously associated with multiple cancer types (Lawrence et al., 2014; Soda et al., 2007; Wagle et al., 2011) and is an activating mutation in many types of lung cancer (Wagle et al., 2011). We hypothesize that the ALK mutation could be stimulating growth, thereby explaining why the cells in the secondary resistant group are resistant to lapatinib. (3) DM331 is an exception in that it is homozygous for 
$\mathrm{BRAF}^{\mathrm{V} 600 \mathrm{E}}$ yet it is by far the most resistant cell line to PLX4720 treatment. Additionally, its expression profile at the basal state most closely associates it with A375, one of the most sensitive lines. However, A375 resistant lines have been generated multiple times, indicating that its sensitivity is not immutable (Abel et al., 2013; Johannessen et al., 2013). We find NRG1, an EGFR family ligand is highly upregulated in DM331 only, and not in A375. On the other hand, addition of NRG1 to A375 causes resistance to PLX4720. Combined with the robust expression of EGFR in DM331 (Roller et al. In prepartion) this may explain the profound resistance of DM331 to BRaf inhibition.

\subsubsection{Adaptive responses to BRaf inhibition.}

We observed broad upregulation of ErbB pathway gene expression 8 hours after PLX4720 treatment, only in the group of cell lines that were sensitive to the combination of PLX4720 and lapatinib. This result is consistent with that of other groups that have found RAS (Nazarian et al., 2010), ERBB3 (Abel et al., 2013; Held et al., 2013; Kugel et al., 2014) and other RTKs (Konieczkowski et al., 2014; Müller et al., 2014) upregulated in BRAF ${ }^{\mathrm{V} 600 \mathrm{E}}$ melanoma in response to PLX4720 treatment.

Surprisingly, we observed a relatively strong downregulation of oncogenic phosphoprotein levels after 8 hours of PLX4720 treatment in CGD and CGE cell lines with a similar but much weaker downregulation of the same proteins in CGA and CGC. We hypothesize that the downregulation observed at the protein level is the direct result of PLX4720 treatment followed by delayed, compensatory signaling in the combination and PLX4720 sensitive cell lines. As partial confirmation of this, we found that ERBB4 total protein and VEGFR phosphorylation levels were 
anticorrelated with PLX4720 cytotoxic response across cell lines. This indicates that treatment with PLX4720 induces receptor tyrosine kinase expression, most likely as a compensatory survival mechanism in lines resistant to treatment as demonstrated in other studies (Abel et al., 2013; Kugel et al., 2014). This also potentially explains the synergy between PLX4720 and lapatinib that we observe in a subset of cell lines. Not only is ERBB family inhibition effective via lapatinib, but inhibition of other receptor tyrosine kinases, via masitinib was also an effective inhibitor based on our drug screen (Roller et al., 2016). It is possible that a "threshold" of RTK activity needs to be reached in order to generate resistance, perhaps by activating Ras signaling and MAP Kinase pathway reactivation.

Lastly, it appears that PLX4720 treatment potentially activates ETS family transcription factors and co-factors to upregulate ERBB pathway genes as well as those of other receptor tyrosine kinase signaling pathways. Abel et al. observed (Abel et al., 2013) ERBB3 activation due to vemurafenib treatment; however they saw activation via FOXD3. Chandarlapaty et al. (Chandarlapaty et al., 2011) identified ERBB3 activation in response to AKT inhibition via phosphorylated FOXO3 in cancer cell lines from multiple tumor types, which were resistant to AKT inhibition. However, the correlation between changes in phosphorylated FOXO3 induced by PLX4720 and PLX4720 cytotoxicity in our melanoma lines indicate that FOXO3 phosphorylation is increased in the most sensitive lines suggesting alternative regulatory programs in the $\mathrm{BRAF}^{\mathrm{V} 600 \mathrm{E}}$ melanoma cell lines that we studied. If FOXO3 phosphorylation activates ERBB3 as a compensatory mechanism to BRaf inhibition, we would expect its log2 fold change to be anticorrelated with 
cytotoxic response to PLX4720, similar to the response of ERBB4. We do see FOXO4 among the transcription factors whose motifs are enriched at the promoters of upregulated ERBB pathway genes. Importantly, while previous studies have observed ERBB3 upregulation in response to various inhibitors (Abel et al., 2013; Chandarlapaty et al., 2011; Zhang et al., 2013), we find broad upregulation of ERBB family members in response to PLX4720 treatment.

It has been noted that multiple receptor tyrosine kinases confer resistance to many forms of treatment (Shtivelman et al., 2014). We find that inhibition of BRaf in melanoma cell lines leads to compensatory signaling via ERBB family members, which are RTKs. Consequently, we and others (Held et al., 2013) find the drug combination vemurfenib and lapatinib to be highly synergistic in a subset of $\mathrm{BRAF}^{\mathrm{V} 600 \mathrm{E}}$ melanoma cell lines. Additionally, we have observed that inhibition of other RTKs, also elicits a synergistic response when combined with BRaf inhibition. Taken together, our results suggest RTK mediated signaling is an adaptive response to mutant BRaf inhibition in melanomas and suggests combination therapy with specific RTK inhibitors.

\subsection{METHODS}

\subsubsection{Cell Culture and Generation of Transcriptome, Proteome and Exome Data}

\subsubsection{Cell culture} SKMEL2, SKMEL24, SKMEL28 cells (American Type Culture Collection), A375, HT144, VMM5A, VMM39, VMM17, VMM18, VMM12, VMM15, DM13, DM6

DM122, DM331 (kind gift from Dr. Craig Slingluff, University of Virginia, 
Charlottesville, VA), and SLM2 (kind gift from Dr. Angela Zarling, University of Virginia) were propagated in RPMI-1640 (Invitrogen) supplemented with 5\% or 10\% FBS (Gemini Bio-Products). All cultures were maintained in a humidified chamber at $37^{\circ} \mathrm{C}$ with $5 \% \mathrm{CO}_{2}$.

\subsubsection{Cytotoxicity assays}

Four hours after being plated in 96-well plates, cells were treated with inhibitors or vehicle control in phenol red-free RPMI-1640 (Invitrogen) without FBS and incubated for 3 days at $37^{\circ} \mathrm{C}$. alamarBlue (Invitrogen) was added to wells and incubated for 4 hours at $37^{\circ} \mathrm{C}$. Fluorescence was measured at $560 \mathrm{~nm}$ excitation/590 nm emission on a Synergy 2 plate reader (BioTek Instruments). Mean results and SE were calculated for triplicate samples.

\subsubsection{Gene array}

Cells were plated and incubated overnight before being treated, in duplicate, with inhibitors or vehicle control in phenol red-free RPMI-1640 without FBS for 8 hours at $37^{\circ} \mathrm{C}$. Cells were placed on ice and rinsed with cold $1 \times$ PBS. Cells were collected and RNA was isolated using the Qiashredder (Qiagen) and RNeasy Mini Kit (Qiagen). RNA was quantified on the NanoDrop 2000 spectrophotometer (Thermo Scientific) and RNA quality was inspected on a $1 \%$ agarose gel. Biotin labeled RNA was hybridized to Illumina 3'IVT human HT-12 BeadChip arrays.

\subsubsection{Reverse Phase Protein Array}

Cells were plated in p60 dishes and incubated overnight to allow the cells to adhere before being treated with inhibitors or vehicle control in phenol red-free RPMI Medium 1640 without fetal bovine serum for 1, 8, or 24 hours. Cells were washed and lysed in 1:1 2x Sample Buffer:Tissue Extraction Reagent (T-PER) (Life 
Technologies). Following lysis the samples were sonicated and centrifuged to clear. Samples were diluted to requested concentration and sent to the lab of Emanuel (Chip) Petricoin at George Mason University for Reverse Phase Array Analysis. Raw sequencing reads were generated using a combination of Nimblegen v2 and Agilent v1 capture probes.

\subsubsection{Exome Sequencing}

Cells were grown to $80 \%$ confluent, trypsinized, and collected in normal growth media. Cells were centrifuged to pellet, wash with PBS, and centrifuged a second time. DNA was extracted using the DNeasy Blood and Tissue Kit (Qiagen) and quantified on the Nanodrop 2000 spectrophotometer (Thermo Scientific). Exome sequencing was performed by Hudson Alpha Institute for Biotechnology (Huntsville, Alabama).

\subsubsection{Statistical Analysis of Functional Genomics and Genomics Data Illumina microarray data was variance stabilized transformed (Federici et al.,} 2013; Improta et al., 2011) using the lumi Bioconductor package in R (Du et al., 2008). Genes that displayed significant variation in basal gene expression across all twelve cell lines were identified using limma to perform one way ANOVA and derive Benjamini-Hochberg False Discovery Rate (FDR) adjusted p-values (Gentleman et al., 2004; Smyth, 2004) and applying a 0.1\% FDR threshold. Significantly varying genes and cell lines were clustered using the R package pvclust (Suzuki and Shimodaira, 2006) with the Pearson correlation distance measure and average linkage. We assessed the significance of the clusters by performing 1000 iterations of the clustering introducing random variations and assessing how much 
randomness was required to loose a specific branch. Cell lines were also clustered according to cytotoxic response to $125 \mathrm{nM}, 625 \mathrm{nM}$ and $1250 \mathrm{nM}$ of PLX4720 and $1000 \mathrm{nM}, 2000 \mathrm{nM}$ and $4000 \mathrm{nM}$ of lapatinib and a three-by-three dose matrix of the two drugs. We used pvclust with a correlation distance measure and average linkage. We identified genes whose expression was significantly altered within each cytotoxicity group of cell lines in response to PLX4720 treatment using limma to perform paired t-tests whereby untreated and PLX4720 treated samples were paired across all replicates and cell lines within a group and applying a 1\% FDR cutoff. Normalized $\log 2$ reverse phase protein array (RPPA) data was generated using methods described in (Improta et al., 2011). We performed a paired t-test analysis described above (for gene expression data). Log2 fold change of proteins in PLX4720 treated versus untreated cells were then correlated to cytotoxic response of cell lines to each dose of PLX4720 using the Spearman correlation coefficient. We summarized the three correlation coefficients across doses derived for each protein by calculating the average value.

\subsubsection{Statistical Analysis of Exome Data}

Exome probes were genotyped using the Genome Analysis Toolkit and the HG19 build as a reference genome. Single nucleotide variants were annotated by SeattleSeq. Genes with novel variants were identified by removing variants previously identified in dbSNP, or 1000Genomes. Novel variants were further filtered by selecting only nonsense and missense mutations as identified by SeattleSeq. The list of genes containing novel nonsynonymous variants were selected by retaining only those that were identified by (Lawrence et al., 2014) as 
being significant drivers across multiple cancers. The list of novel, nonsynonymous variants located in these genes were input to PolyPhen2 to determine the potential functional severity of these nonsynonymous variants. Genes were identified that uniquely contained variants in each cell line, and within each cytotoxicity group.

\subsubsection{Pathway Enrichment Analysis}

Gene sets found to be significant underwent pathway enrichment analysis via Pathway Express and Gene Set Enrichment Analysis (GSEA) using MSigDB datasets. Significant gene sets and pathways were identified as having a FDR enrichment value of 0.05 for both tools. To ascertain significance of transcription factor binding motifs, we identified motifs unique to the CGC. For motifs not unique to the CGC, we summed the rank of CGC with 100 minus the rank of the other group. When multiple groups contained the motifs, we averaged the ranks, then summed the rank of CGC with 100 minus the averaged rank. 


\subsection{ACKNOWLEGDEMENTS}

This chapter was adapted from Systems Analysis of Adaptive Responses to MAP Kinase Pathway Blockade in BRAF Mutant Melanoma (Capaldo et al., 2015). 


\section{Synergistic combination treatment of a subset of melanoma}

\section{involves cotargeting of the MAPK pathway and PI3K/AKT}

\section{signaling pathway via receptor tyrosine kinase inhibition}

\subsection{ABSTRACT}

The majority of BRAFV600E melanomas demonstrate either acquired or intrinsic resistance to vemurafenib therapy. The mechanisms of resistance can be attributed in part to adaptive responses used by melanoma cells to overcome the inhibition. Overcoming the adaptive response to vemurafenib therapy is of paramount importance for the treatment of melanoma. We observe that the combination of PLX4720, a vemurafenib analog, with secondary drugs associated with the inhibition of receptor tyrosine kinases (RTKs), or the PI3K/AKT signaling pathway shows pronounced ability to overcome the adaptive response. We also observe that combining PLX4720 with these secondary inhibitors leads to individualized responses in each cell line that tend to involve the MAPK and PI3K/AKT signaling pathways via gene expression microarray and reverse phase protein arrays. We were also able to observe that epigenetic manipulation of PLX4720 resistant treatment naïve cell line led to its sensitization to RTK inhibition. Our observations point to the importance of RTKs being used by melanomas to leverage adaptive responses against PLX4720 treatment. 


\subsection{INTRODUCTION}

Approximately $50 \%$ of melanomas containing activating mutations in BRAF respond to BRAF inhibitors such as vemurafenib and dabrafenib (Shtivelman et al., 2014). A therapeutic response to BRAF inhibitors is only observed in $50-60 \%$ of patients, and only $10 \%$ demonstrate a complete response. The responses are not usually durable, with disease relapsing within 6 months (Chapman et al., 2011). Recurrences will often appear at the same site as the original tumor (Wagle et al., 2011), indicating that tumors are rapidly adapting to targeted therapy, even when initial responses are robust. Extending duration of response is possible using a combination of therapies that target MEK and BRAF, but recurrence is still observed in patients (Wagle et al., 2014).

Mechanisms of resistance to BRAF inhibitors have been determined via analysis of patient samples that demonstrate resistance to single agent therapy. (Paraiso et al., 2010; Poulikakos et al., 2011; Shi et al., 2014b; Solit and Rosen, 2011; Spagnolo et al., 2014; Van Allen et al., 2014; Wagle et al., 2014). Resistance mechanisms almost always involve reactivation of the MAPK pathway, although alternative mechanisms involving PI3Kinase, STATs, HIPPO, beta-catenin, BH3 proteins, autophagy and translational regulation have also been observed (Atefi et al., 2011; Atkinson et al., 2015; Conway et al., 2011; Corcoran et al., 2013; Davies, 2012; Delmas et al., 2015; Deuker et al., 2015; Goldstein, 2004; Gopal et al., 2014; Hartsough and Aplin, 2013; Hoeflich et al., 2012; Lassen et al., 2014; Lin et al., 2015; Ma et al., 2014; Pelletier et al., 2015; Rebecca et al., 2014; Schneider et al., 2014; Scortegagna et al., 2014, 2015; Shi et al., 2014a; Silva et al., 2014; Thomas et al., 
2014; Yadav et al., 2014). Resistant tumors utilize a host of different mechanisms, as any pathway that activates growth will provide the tumor with a selective advantage. It has been observed that metastases or varying regions of the tumor will often employ different resistance mechanisms (Shi et al., 2014b; Straussman et al., 2012; Van Allen et al., 2014).

In the previous chapter, we observed that among the rapid responses to MAPK blockade, an activation of the ERBB pathway was prominent (Capaldo et al., 2015). A number of investigators (Lito et al., 2013; Rebecca and Smalley, 2014; Solit and Rosen, 2014) have indicated that rapid adaptive responses, such as activation of the ERBB pathway (Abel et al., 2013), are a significant component of intrinsic resistance, and suggest that the adaptive responses drive selection of genetic and epigenetic variants in which the MAPK pathway is reactivated. Therefore, these adaptive responses need to be cataloged and analyzed in order to overcome resistant disease. Cataloging the adaptive response could lead to the identification of novel targets for the purpose of combination therapy, and increase the durability of the therapy. ERBB pathway activation can induce MAPK pathway activation, but also can induce activation of the PI3K pathway. In Chapter 2, we observed an upregulation of NRAS and HRAS gene expression in response to PLX4720 treatment (Capaldo et al., 2015), consistent with the findings of others (Nazarian et al., 2010), which has been shown to activate both the MAPK and PI3K signaling pathways (Fattore et al., 2013; McCubrey et al., 2006). Given the recurrence observed in patients treated with combinations that retarget the MAPK pathway (Wagle et al., 2014), it is clear that additional combinatorial strategies are required. 
Here, we show that synthetic lethal screening using the Bliss model for synergy is an effective means for identifying promising combinations. Assaying these combinations using high-throughput methods also allows for the identification of additional targets for combinations, mechanisms of resistance, and mechanisms of synergy. In our further study of melanoma, we demonstrate that targeting members of the PI3K signaling pathway can inhibit the adaptive response of PLX4720 treatment. Many have observed that PI3K signaling plays an important role in melanoma resistance (Carnero et al., 2008; Villanueva et al., 2010), and in this chapter we profile this response in a functional genomics manner. Through this methodology, we are able to quantify the ablation of the adaptive response across multiple readouts of the pathway.

The Weber lab conducted a targeted combinatorial chemical genetic screen using as primary drugs either the vemurafenib analog PLX4720 or two other inhibitors of the MAPK pathway in two-way combinations with 58 drugs or clinically relevant tool compounds in $12 \mathrm{BRAF}^{\mathrm{V} 600 \mathrm{E}}$ melanoma cell lines (Roller et al., 2016). We observed that half the lines showed synergistic benefit by combining lapatinib or masitinib with PLX4720. Importantly, the lines that showed benefit from this combination were those that were least sensitive intrinsically to BRAF inhibition, indicating the importance of Receptor Tyrosine Kinase (RTK) signaling in vemurafenib resistance, as shown previously (Capaldo et al., 2015; Held et al., 2013; Langdon et al., 2015; Nazarian et al., 2010; Wilson et al., 2011, 2012; Yadav et al., 2012). Effective drug combinations varied for each cell line, indicating that the "wiring" of the signaling network and mechanisms of adaptive resistance differed 
for each line even though all were driven by BRAFV600E and 6 of the 12 showed benefit from an RTK inhibitor. Protein pathway phosphorylation/activation mapping via reverse phase protein arrays (RPPA) and gene expression analysis confirmed that, even when cells were sensitive to the combination of PLX4720 and lapatinib, the adaptive changes in intracellular signaling in response to BRAF inhibition differed and the mechanism(s) by which lapatinib or masitinib were synergistically cytotoxic differed. We propose that intrinsic and adaptive resistance to BRAF inhibition in BRAFV00E melanomas occurs by multiple mechanisms that differ substantially, dependent on the broader genetic and epigenetic landscape of the cancer cells that shape the underlying architecture of cell signaling networks. Because Receptor Tyrosine Kinases can activate multiple resistance pathways, inhibitors of these receptors can play an important role in drug combinations in a variety of genetic backgrounds and may be more useful in drug combinations than inhibitors of individual intracellular signaling pathways.

To investigate the epigenetic landscape of our resistant melanoma lines, we utilized DNA methylation microarrays. DNA methylation is an epigenetic mechanism thought to stabilize the silencing of genes (Thomas et al., 2014) and has been observed to have functional significance in colorectal, gastric, and breast cancers (de Araújo et al., 2015; Gazzoli et al., 2002; Pinheiro et al., 2010; Venkatachalam et al., 2010; Wong et al., 2011). Limited work has been done in melanoma with regards to DNA methylation state, but preliminary studies suggest that it plays a significant role in mechanisms of resistance (de Araújo et al., 2015). It is uncertain as to whether the adaptive response to vemurafenib treatment utilizes changes in DNA 
methylation, but it has been observed that DNA methylation profiles are associated with clinical outcomes (Thomas et al., 2014). Multiple studies have observed that chronic treatment with vemurafenib leads to upregulation of RTK in melanoma cell lines (Konieczkowski et al., 2014; Müller et al., 2014; Thomas and Erickson, 2009). Our study of DNA methylation in melanoma showed that RTK pathways displayed a loss of DNA methylation at their promoters, indicating that DNA methylation also contributes to the activation of RTK signaling, which itself feeds into the reactivation of the MAPK pathway. Taken together, these studies give further credence to the importance of targeting the adaptome at multiple points to further increase durability of the response.

\subsection{RESULTS}

\subsubsection{High throughput cytotoxicity screening of melanoma reveals synergistic combinations for treatment of cancer}

As described in the previous chapter, we utilize a synthetic lethal screen in a three by three dose format to identify synergistic combinations of targeted therapies for further exploration in preclinical models. In a screen of the BRAFV600E melanomas, we profiled compounds including a panel of MAPK pathway inhibitors; PLX4720, a BRAFV600E inhibitor; RAF265, an inhibitor that targets BRAFWT, BRAFV600E, CRAF, and has activity against VEGFR2; and PD325901, a MEK1/2 inhibitor with a host of secondary drugs (Table 3.1). All three primary drugs yielded combinations that displayed synergistic activity in vitro. Additionally, 14 of the secondary drugs demonstrated synergy with all three primary drugs in at least one cell line (Table 3.2). Performance of the combinations was assayed using the Bliss 
difference (Zhao et al., 2014) as our synergy score. Each combination was assayed

across a 3 × 3 dose response matrix.

Table 3.1 Drugs used in melanoma screen, the targets, and the suppliers

\begin{tabular}{|c|c|c|}
\hline Drug & Target & Supplier \\
\hline AMPKi, compound C & AMPK & VWR \\
\hline AZD-1152 & Aurora kinase & Selleck \\
\hline BEZ235 & PI3K & Selleck \\
\hline Bortezomib & Proteasome & LC Laboratories \\
\hline Caffeine & PDE & VWR \\
\hline Camptothecin & Topoisomerase I & Sigma \\
\hline Celecoxib & $\operatorname{cox} 2$ & VWR \\
\hline CGP57380 & MNK1/2 & VWR \\
\hline Chloroquine & Endosome & VWR \\
\hline CP-690550 & JAK3 inhibitor & LC Laboratories \\
\hline Curcumin & Broad & VWR \\
\hline D609 Pro-drug & Phospholipase C & LC Laboratories \\
\hline Dasatinib & $\mathrm{SRC}$ and $\mathrm{BCR} / \mathrm{ABL}$ & VWR \\
\hline Debromohymeniadlisine (DBH) & CHK1/2 & Alexis \\
\hline Diclofenac Sodium & COX 1 and 2 & VWR \\
\hline DNA-PK inhibitor & DNA-PK & VWR \\
\hline Doxorubicin & Topoisomerase II & Sigma \\
\hline EGCG & Broad & Sigma \\
\hline Etoposide & Tubulin disassembly & Sigma \\
\hline Farnesylthiosalicylic acid (FTS) & Ras & Cayman Chemicals \\
\hline FK506 & Calcineurin & Sigma \\
\hline Flavopiridol & CDK & Enzo \\
\hline Gefitinib & EGFR & LC Laboratories \\
\hline Geldanamycin & Hsp 90 & VWR \\
\hline Go6976 & PKC & VWR \\
\hline H-89 & PKA & Sigma \\
\hline HA-1077 dihydrochloride & ROCK & VWR \\
\hline Imatinib & Abl1, kit, PDFR & LC Laboratories \\
\hline Indirubin & GSK3 & VWR \\
\hline Lapatinib & EGFR and HER2/neu & LC Laboratories \\
\hline Masitinib mesylate (AB1010) & PDGF, FGFR3, ckit & LC Laboratories \\
\hline Nilotinib & BCR-abl & LC Laboratories \\
\hline OSU-03012 & PDK & LC Laboratories \\
\hline Paclitaxel & Tubulin disassembly & LC Laboratories \\
\hline PD173074 & FGFR & VWR \\
\hline PD325901 & MEK & Pfiser \\
\hline PF-562271 & FAK & Selleck \\
\hline PI-103 & PIK3CA & Selleck \\
\hline PJ34 & Parp & VWR \\
\hline Picropodophyllotoxin (PPP) & IGF1-R & Sigma \\
\hline PLX4720 & BRaf & gift from Plexxicon \\
\hline Raf265 & Raf & Novartis \\
\hline Roscovitine (CYC-202) & CDK2, CDK1, CDK5 & LC Laboratories \\
\hline S3I-201 & STAT3 & VWR \\
\hline Suberoylanilide Hydroxamic Acid (SAHA) & HDAC & gift from Dr. David Jones, UVA \\
\hline $\begin{array}{l}\text { SANT-1 } \\
\end{array}$ & Hedgehog & VWR \\
\hline SB-202190 & p38-alpha & LC Laboratories \\
\hline SB-431542 & TGFBR1/ALK5, ACVR1B/ALK4 & Sigma \\
\hline SC-514 & IKK2 & VWR \\
\hline Bosutinib (SKI-606) & Tyrosine kinase & LC Laboratories \\
\hline Sorafenib & Braf, VEGFR-2 and PDGFR-CE $\leq$ & LC Laboratories \\
\hline SP600125 & JNK & LC Laboratories \\
\hline Staurosporine (K-252c) & PKC, PKA, PKG & LC Laboratories \\
\hline Sunitinib & KIT, VEGFR2, FLT3 & LC Laboratories \\
\hline Temsorilimus (CCI-779) & mTOR & LC Laboratories \\
\hline Trichostatin A (TSA) & HDAC & Selleck \\
\hline Vatalanib & VEGFR-1 and VEGFR-2 & LC Laboratories \\
\hline VX-680/MK-0457 & Aurora kinases, $A B L$ kinase & LC Laboratories \\
\hline WP-1066 & STAT5 & Selleck \\
\hline Y27632 & Rho kinase & Tocris \\
\hline
\end{tabular}


Table 3.2 Secondary drugs that demonstrated synergy in at least one cell line when combined with one or more of the MAPK inhibtors

\begin{tabular}{|c|c|}
\hline Secondary Drug & Putative Targets \\
\hline AMPKi & AMPK \\
\hline Staurosprine & PKC, PKA, PKG \\
\hline Lapatinib & EGFR \\
\hline Masitinib & PDGF, FGFR3, c-kit \\
\hline OSU-03012 & PDK \\
\hline PI-103 & PI3KCA \\
\hline PJ34 & PARP \\
\hline Roscovitine & CDK 1, 2, 5 \\
\hline SAHA & HDAC \\
\hline SB-202190 & p38/SAPK \\
\hline Temsirolimus & mTOR \\
\hline Vatalanib & VEGFR1 and 2 \\
\hline Caffeine & PDE \\
\hline Indirubin & GSK3 \\
\hline
\end{tabular}

To determine patterns of response across combinations, we took the

maximum observed synergy score across all doses for each line and allowed these maximum synergy scores to undergo unsupervised hierarchical clustering using the correlation distance (Figure 3.1). In total, we clustered 109 combinations. We observed that PLX4720 was effective in combination with masitinib, another RTK inhibitor like lapatinib; and with OSU-03012, an inhibitor of PDK. Lines that demonstrated synergy between PLX4720 and lapatinib also tended to demonstrate synergy between PLX4720 and masitinib, and PLX4720 and OSU-03012. PI-103, a PI3K inhibitor, combined with any of the primary drugs also demonstrated in most cases the synergy with observed in the aforementioned combinations. 

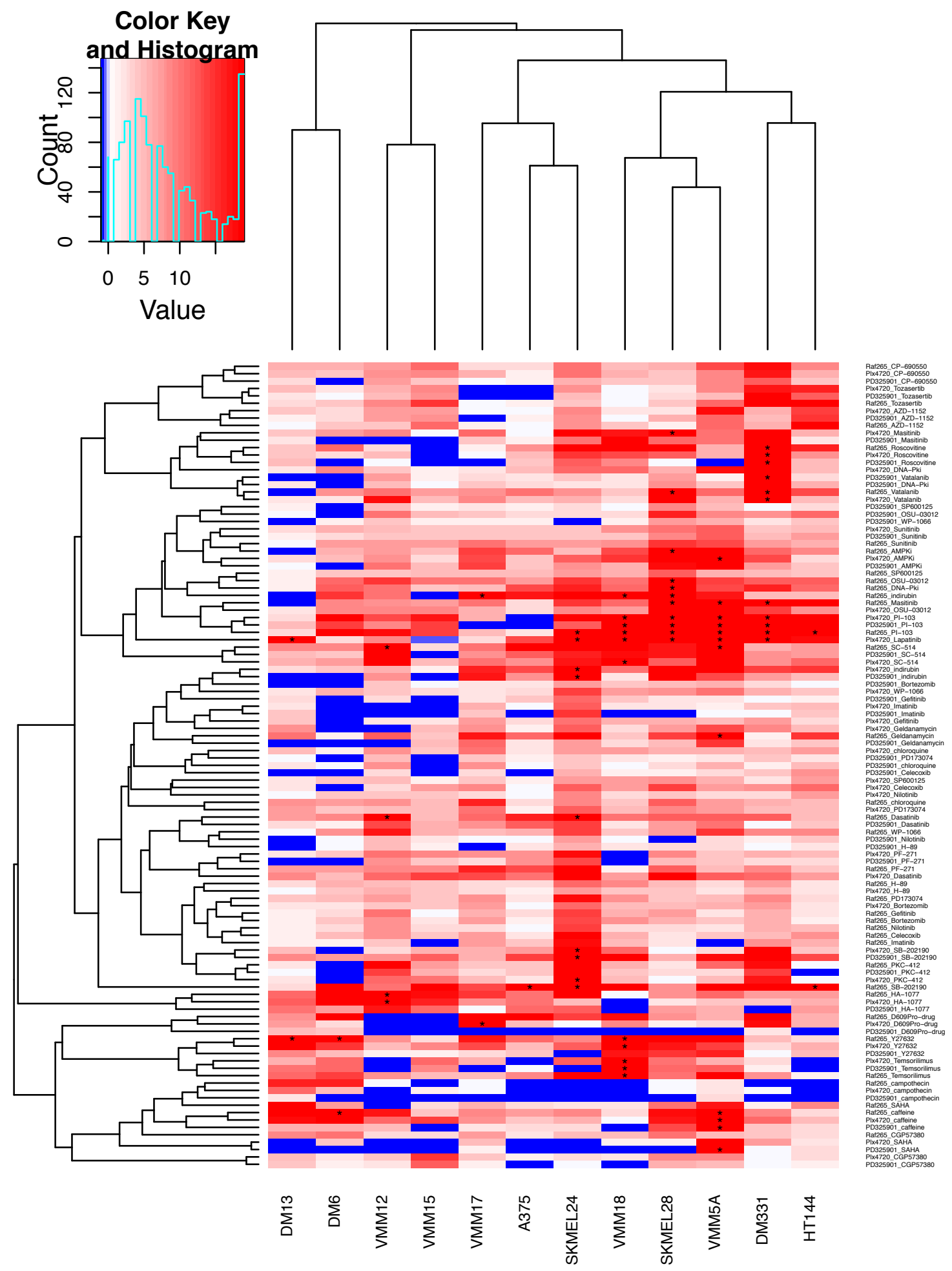

Figure 3.1 Synthetic lethal screen identifies synergistic combinations in melanomas 
Heat map of maximum observed synergy scores for BRAFV600E melanoma cell lines ( $x$ axis) for 109 combinations (y-axis). Synergy scores of $26 \%$ or greater are denoted with an "*". Clustering for cell lines and combinations was done using correlation distance and average linkage. Data from the screen was generated by Devin Roller, in the Weber lab at the University of Virginia.

When we examined the effectiveness of combinations across the panel of cell lines, we observed multiple combinations that yielded synergistic effects in the same lines. Given that PI3K, RTKs, and PDK are all constituents of the AKT pathway (Carnero et al., 2008; McCubrey et al., 2006), and that combining MAPK pathway inhibition with inhibition of any one of these constituents is synergistic, would suggest strongly that AKT signaling is a key adaptive response to MAPK pathway inhibition. This would seem to indicate that basal levels of AKT signaling, and more generally, RTK signaling, may contribute to the level of resistance to MAPK pathway inhibition.

\subsubsection{Basal signaling activity of the resistant lines when assayed by reverse phase protein arrays does not significantly differ from the lines} sensitive to PLX4720

To determine the level of basal RTK signaling in our panel, we utilized

reverse phase protein arrays (RPPA) to quantify protein levels in individual cell lines. We utilized one way ANOVA to determine epitopes on our array that were differentially abundant across our lines at an FDR of 1\% (Figure 3.2). Using a review of the literature, we annotated epitopes that belong to the PI3K and MAPK signaling pathways given their significance in the adaptive response to MAPK blockade. We were able to identify some differential activity between the resistant and sensitive lines in the PI3K pathway, but these differences are not maintained across all of the resistant lines and sensitive lines, respectively. Given the lack of significant basal 
variation in the cell lines, we sought to determine if post treatment differences tracked with sensitivity to PLX4720. 


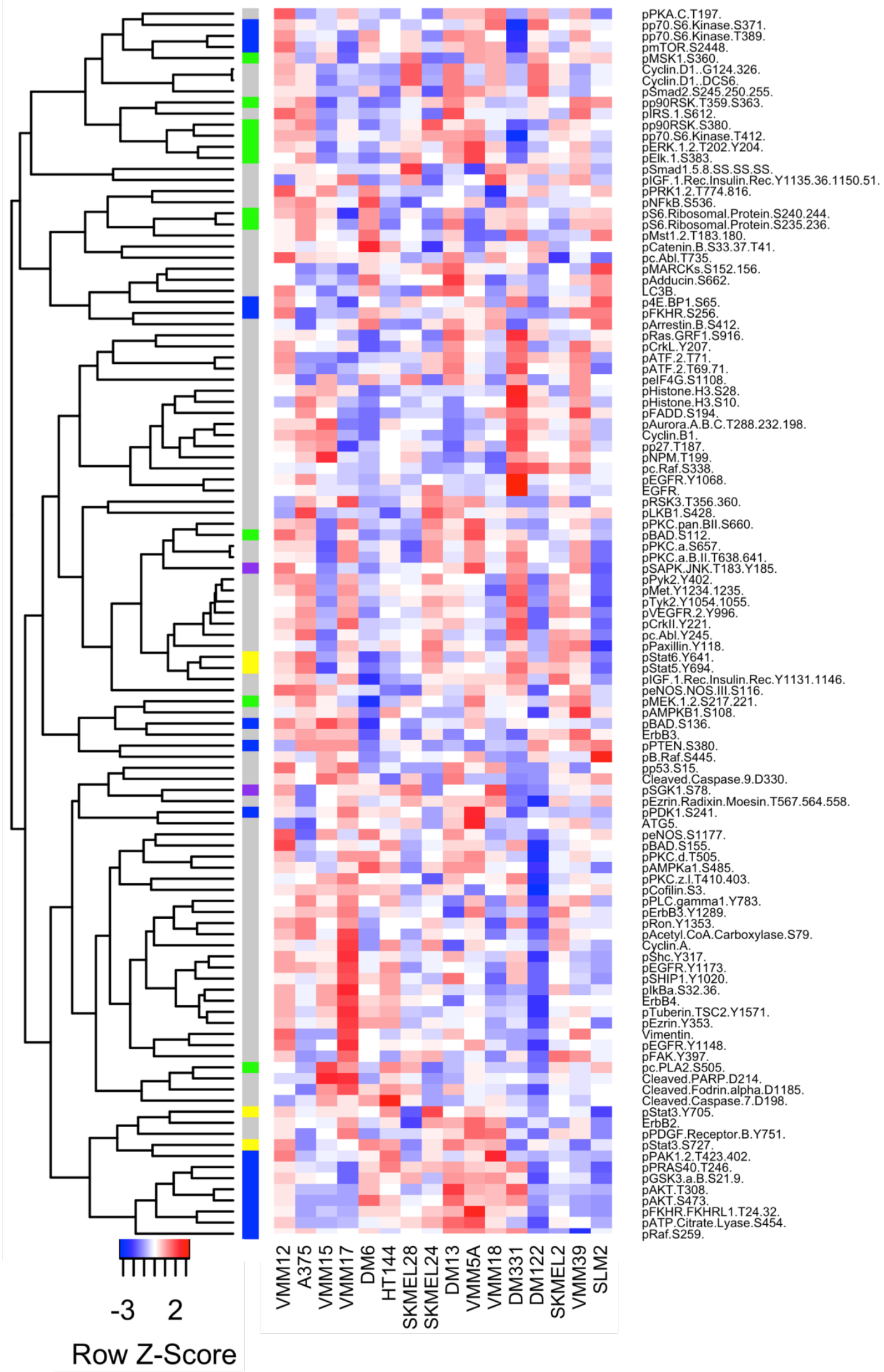




\section{Figure 3.2 Heat map of one way ANOVA analysis of basal phospho-epitopes determined by RPPA.}

Using a one way ANOVA test, we selected for epitopes that varied between cell lines at a FDR of 1\% or better. Unsupervised hierarchical clustering of row scaled epitopes' intensities (y-axis) using correlation distance and average linkage is shown. Cell lines were ordered from left to right by decreasing sensitivity to PLX4720. Unsupervised clustering using correlation distance and average linkage of their epitopes' intensities did not order cell lines in proportion to drug sensitivity or driver oncogene. Pathway membership is denoted along the $y$-axis by the presence of colored boxes for the MAPK pathway (Green), PI3K pathway (Blue), stress (Purple), or STAT signaling (Yellow).

We determined the changes induced in the PI3K and MAPK pathway after treatment with PLX4720 across all cell lines by calculating the fold change response to PLX4720 treatment 8 hours after exposure and plotting the responses for each cell line as a box plot (Figure 3.3, Panel A). This allowed us to observe the distribution of effects due to treatment across the entire pathway. We observed a weak correlation between responses in the MAPK pathway and sensitivity to PLX4720, and we saw no evidence that responses in PI3K epitopes can differentiate sensitive and resistant lines at 8 hours after exposure. Based on our gene expression study (Chapter 2), we observed that many transcriptional responses were only beginning to occur around 8 hours. Given these results, we sought to determine whether treatment effects investigated at earlier and later time points could serve to better differentiate sensitive and resistant lines, as well as determine what effects the combination treatment had on responses. 

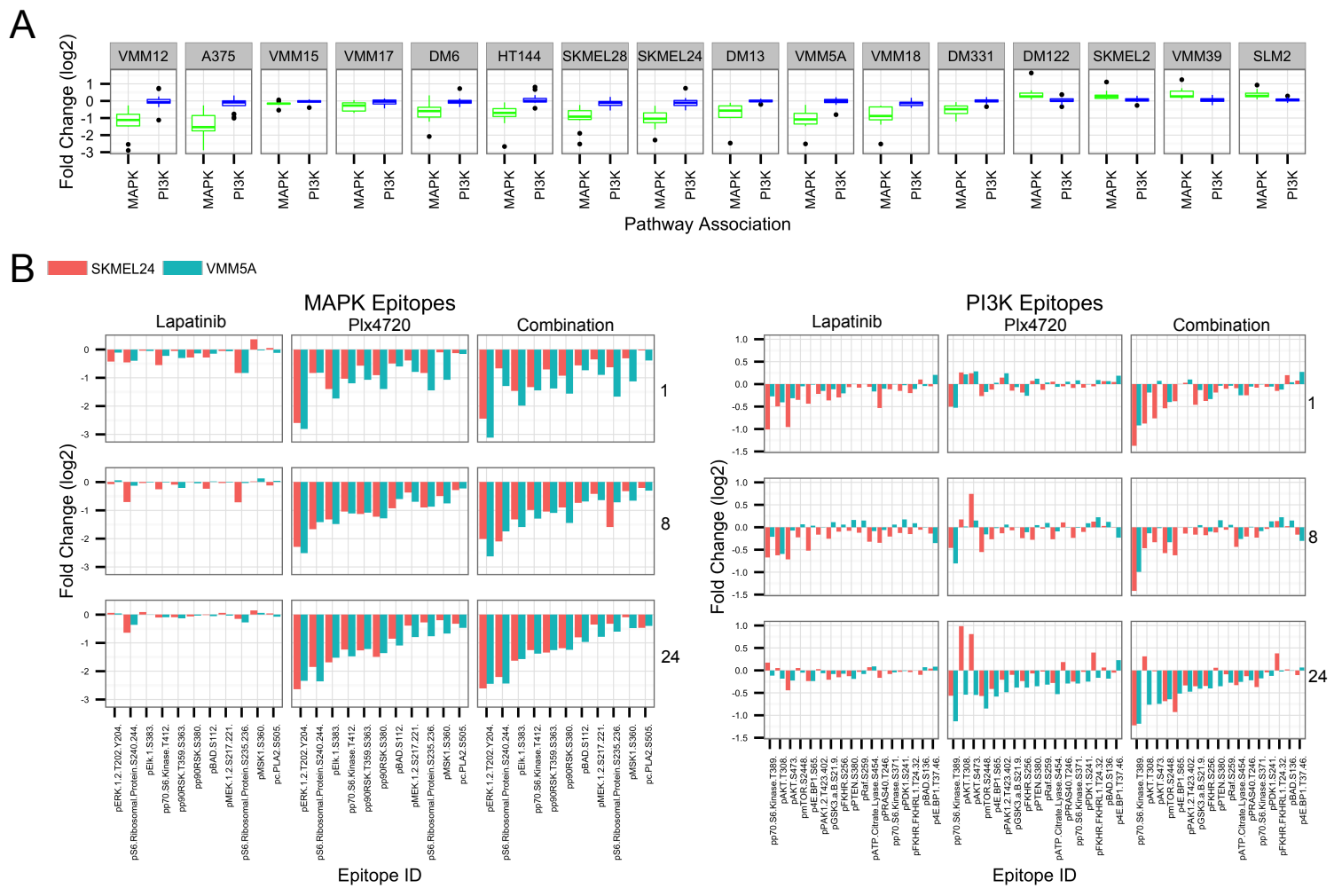

\section{Figure 3.3 Changes in PI3 Kinase and MAP Kinase pathway protein phosphorylations in response to drug treatment.}

A. Whisker plot of normalized, log2 transformed RPPA fold changes (PLX4720 treated over untreated) were plotted for MAPK pathway (green) and PI3K pathway (blue) epitopes for 12 BRAF mutant and 4 BRAF wt melanoma cell lines. Epitopes were selected from the arrays that were determined to be associated with the MAPK or PI3K signaling pathways, as shown in Panel B. Lines are ordered from most to least sensitive to PLX4720 treatment by IC50. B. Normalized, log2 transformed RPPA fold changes (drug treated over untreated) were plotted for SKMEL24 (red) and VMM5A (blue). Epitopes were selected from the arrays that were determined to be associated with the MAPK signaling pathway (left) or PI3K signaling pathway (right). Each facet of the plot represents the fold changes induced by treatment with lapatinib, PLX4720, or the combination (left to right) after 1, 8, or 24 hours (top to bottom). Samples were prepared by Devin Roller, in the Weber lab at the University of Virginia

Again using RPPA analysis, we profiled responses at time points of 1, 8, and 24 hours after treatment with lapatinib alone, PLX4720 alone, or the combination (Figure 3.3, Panel B). We chose to highlight two heterozygous cell lines, SKMEL24 and VMM5A, because both of these lines demonstrate synergy when exposed to the combination of PLX4720 and lapatinib and they show different levels of resistance 
to PLX4720. VMM5A is among the most resistant melanoma cell lines; the resistance of SKMEL24 is close to the average resistance observed in our studies. We began by looking at the RPPA responses to the treatment course in the MAPK pathway. In SKMEL24, lapatinib had no significant effect on the epitopes that map to members of the MAPK signaling pathway. When exposed to PLX4720 alone, both lines showed a marked downregulation of the phosphorylation levels in the MAPK pathway, with very slight differences between the lines in the magnitudes of their responses.

We next examined the response of the PI3K signaling pathway to the combination treatment course over time. The effects of lapatinib treatment appeared to subside fairly quickly in SKMEL24; by 24 hours there was limited perturbation of the phosphorylation level compared to the drastic downregulation of phosphorylations at 1 and 8 hours in these cells. VMM5A shows very subdued responses to lapatinib treatment at all time points. Phospho AKT on threonine 308 is the most drastic response we observe under treatment with lapatinib in SKMEL24. It rapidly loses phosphorylation at 1 hour, maintains the loss in phosphorylation at 8 hours, and then drops back to approximately basal levels of phosphorylation by 24 hours. This indicates that AKT phosphorylation is strongly dependent on lapatinib treatment.

Treatment with PLX4720 generated downregulation of a battery of phosphorylation sites in both lines at 1 hour, and this continued to drop for the duration of the assay. The battery of phosphorylation sites in VMM5A is more strongly inhibited compared to the inhibition of these sites in SKMEL24, but the trends are similar. This battery of phosphorylation sites is even more strongly 
downregulated under exposure to the combination, and the response of SKMEL24 at 24 hours now matches the magnitude of the response of VMM5A to PLX4720 treatment at 24 hours. Additionally, the early phospho AKT response to lapatinib treatment in SKMEL24 is unable to recover as we saw with cells treated solely with lapatinib. VMM5A also shows a substantial reduction of AKT phosphorylation at all time points. The RPPA data serve to show that the combination of PLX4720 and lapatinib strongly inhibits the adaptive responses we observe under the conditions for single drug treatment. Having observed the evidence in the RPPA response at 24 hours, we sought to determine if we could isolate the adaptive response at the level of gene expression at 8 hours.

\subsubsection{Cell lines show varying mechanisms of response after treatment with single drugs or the combination. \\ The RPPA data at 24 hours suggests that synergistic effects can block the} previously observed adaptive response. To investigate the effects of lapatinib and masitinib in combination with PLX4720 we profiled the gene expression responses in five lines to each of the treatments 8 hours after exposure (Figure 3.4). Unsupervised hierarchical clustering shows that each line responds uniquely compared to any other line. A375 showed an extremely robust response to treatment with PLX4720, which was expected given its strong sensitivity to the drug. It showed very limited response to either of the secondary drugs alone, and the addition of either lapatinib or masitinib ablates some of the responses to PLX4720 treatment alone. HT144 showed little response except when exposed to masitinib either alone or in combination with PLX4720. DM331, too, showed very few significantly affected genes, however, it demonstrated a stronger response than 
HT144. SKMEL24 and SKMEL28 showed the most robust responses to PLX4720

treatment alone and to the combinations. As with the other lines, very limited response is observed to treatment with either of the secondary drugs alone. 

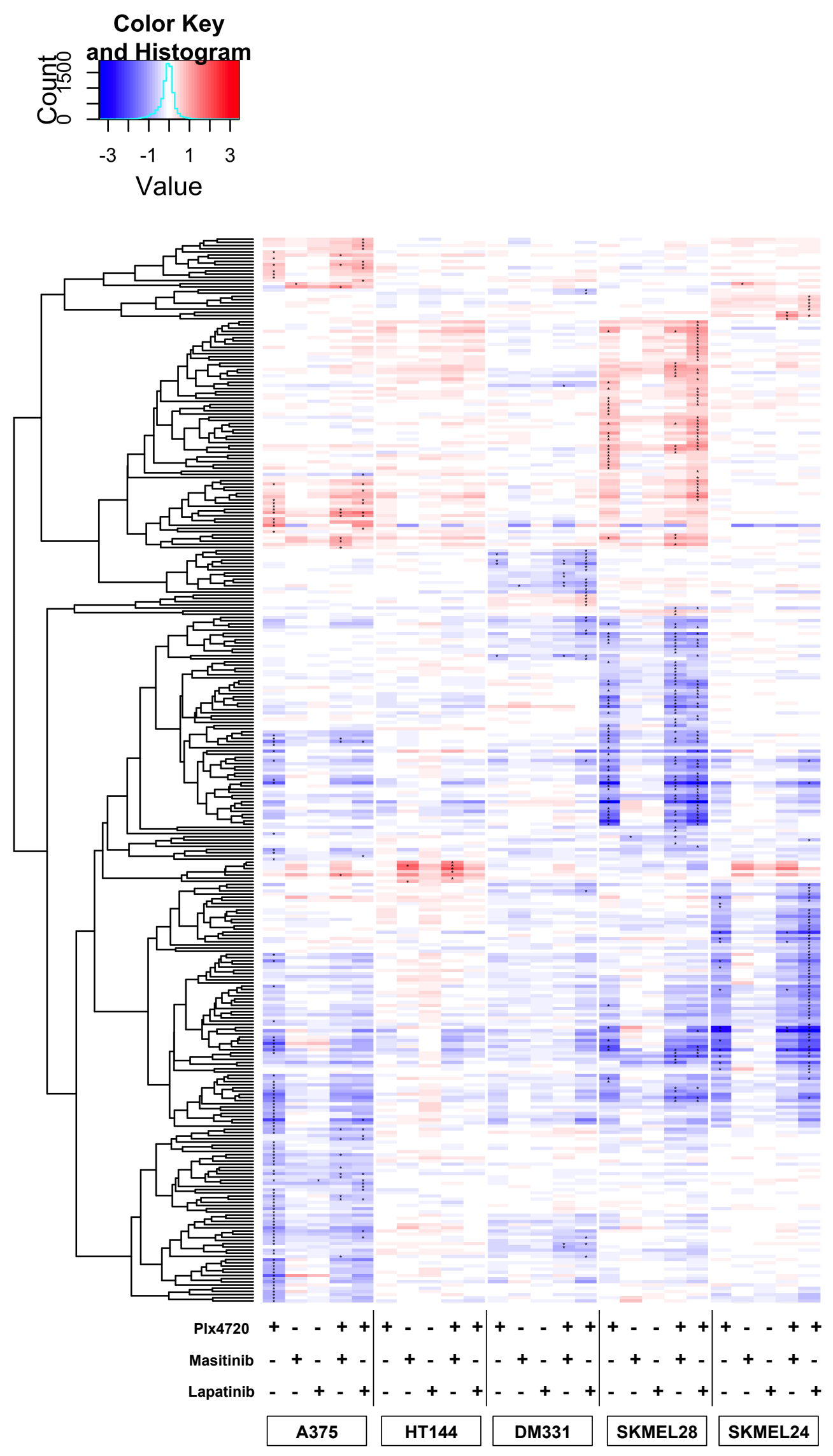
Figure 3.4 Each line shows unique pattern of transcriptional responses to single and combination treatment for PLX4720, masitinib, and lapatinib

Using a moderated $t$-test, we identified differentially expressed genes 8 hours after treatment. Log2 fold change response for genes (y-axis) underwent unsupervised hierarchical clustering using correlation distance and average linkage. Cell lines were exposed to treatment with PLX4720 alone (PvC), masitinib alone (MvC), lapatinib alone $(\mathrm{LvC})$, the combination of PLX4720 and masitinib (PMvC), or the combination of PLX4720 and lapatinib (PLVC). Genes with an FDR better than 1\% are denoted with “*”. Samples were prepared by Devin Roller, in the Weber lab at the University of Virginia

It appears that each line demonstrates different blocks of genes responding to the various treatments. Looking at each of the clusters, each line's response occupies an individual cluster; few genes demonstrated significant responses across multiple lines. SKMEL24 is the line most responsive across all treatments and has the highest number of significant expression changes of any of the lines, both upregulated and downregulated in response to treatment. Among this panel of cell lines, SKMEL24 is the only line that is heterozygous for the BRAF V600E allele. Our finding (Chapter 2) that zygosity of the BRAF allele influences the ability of a line to signal adaptively, may explain why SKMEL24 is showing a more robust adaptive response than the other lines. However, SKMEL24, HT144, and A375 are all part of the same cytotoxicity group; DM331 and SKMEL28 are in CGB and CGC respectively.

The combination of PLX4720 with lapatinib or masitinib appears strongly to amplify the transcriptional response to PLX4720 treatment alone. It would appear that the synergistic mechanisms of BRAF and RTK inhibition act in concert to ablate the adaptive response observed in the previous chapter. To investigate further whether the combination treatment blocks the adaptive response, we took the list of differentially expressed genes in response to either combination treatment for each 
line through a pathway enrichment analysis using MSigDB (Table 3.3).

Unsurprisingly, A375 showed no pathways enriched for its set of differentially expressed genes in response to combination treatment. HT144 showed only four pathways, and none of them are associated with any of the responses we have been discussing. DM331, SKMEL24, and SKMEL28 all show indications that MAPK signaling, ErbB signaling, and p53 signaling are affected by the combination treatment. These pathway results suggest that combination treatment is able to block the adaptive response. The proximity of the targets of the drugs appears to be influencing the response to treatment. As previously discussed, ErbB signaling induces MAPK pathway signaling and PI3K/AKT signaling; in our screen, we do observe that inhibition of PDK, a component of the PI3K/AKT pathway, can act as an effective secondary drug target.

Table 3.3 MSigDB pathway enrichment of differentially expressed genes in response to combination treatment of PLX4720 with either lapatinib or masitinib

\begin{tabular}{|c|c|c|c|c|}
\hline Gene Set Name & Description & $\begin{array}{l}\text { Enrichment } \\
\text { FDR q-value }\end{array}$ & Genes From Gene List In Gene Set & Cell Line \\
\hline $\begin{array}{c}\text { REACTOME_CHOLESTEROL_BIOS } \\
\text { YNTHESIS }\end{array}$ & $\begin{array}{c}\text { Genes involved in } \\
\text { Cholesterol } \\
\text { biosynthesis }\end{array}$ & $3.80 \mathrm{E}-10$ & HMGCS1|MVK|MSMO1|SQLE & HT144 \\
\hline $\begin{array}{l}\text { REACTOME_METABOLISM_OF_LI } \\
\text { PIDS_AND_LIPOPROTEINS }\end{array}$ & $\begin{array}{l}\text { Genes involved in } \\
\text { Metabolism of } \\
\text { lipids and } \\
\text { lipoproteins }\end{array}$ & $3.81 \mathrm{E}-05$ & HMGCS1|MVK|MSMO1|SQLE & HT144 \\
\hline $\begin{array}{c}\text { KEGG_TERPENOID_BACKBONE_BI } \\
\text { OSYNTHESIS }\end{array}$ & $\begin{array}{c}\text { Terpenoid } \\
\text { backbone } \\
\text { biosynthesis }\end{array}$ & $4.28 \mathrm{E}-04$ & HMGCS1|MVK & HT144 \\
\hline KEGG_STEROID_BIOSYNTHESIS & $\begin{array}{c}\text { Steroid } \\
\text { biosynthesis }\end{array}$ & $4.28 \mathrm{E}-04$ & MSM01|SQLE & HT144 \\
\hline PID_ERBB_NETWORK_PATHWAY & $\begin{array}{c}\text { ErbB receptor } \\
\text { signaling network }\end{array}$ & $5.72 \mathrm{E}-07$ & HBEGF |NRG1|EREG|TGFA & DM331 \\
\hline NABA_SECRETED_FACTORS & $\begin{array}{l}\text { Genes encoding } \\
\text { secreted soluble } \\
\text { factors }\end{array}$ & $1.79 \mathrm{E}-04$ & HBEGF | NRG1|EREG|TGFA |CSF2 | VEGFC & DM331 \\
\hline $\begin{array}{c}\text { REACTOME_SHC1_EVENTS_IN_ER } \\
\text { BB4_SIGNALING }\end{array}$ & $\begin{array}{l}\text { Genes involved in } \\
\text { SHC1 events in } \\
\text { ERBB4 signaling }\end{array}$ & $1.79 \mathrm{E}-04$ & HBEGF|NRG1|EREG & DM331 \\
\hline $\begin{array}{c}\text { REACTOME_GRB2_EVENTS_IN_E } \\
\text { RBB2_SIGNALING }\end{array}$ & $\begin{array}{l}\text { Genes involved in } \\
\text { GRB2 events in } \\
\text { ERBB2 signaling }\end{array}$ & $1.79 \mathrm{E}-04$ & HBEGF|NRG1|EREG & DM331 \\
\hline
\end{tabular}




\begin{tabular}{|c|c|c|c|c|}
\hline $\begin{array}{c}\text { KEGG_ERBB_SIGNALING_PATHW } \\
\text { AY }\end{array}$ & $\begin{array}{l}\text { ErbB signaling } \\
\text { pathway }\end{array}$ & $1.79 \mathrm{E}-04$ & HBEGF|NRG1|EREG|TGFA & DM331 \\
\hline NABA_MATRISOME & $\begin{array}{c}\text { Ensemble of genes } \\
\text { encoding } \\
\text { extracellular } \\
\text { matrix and } \\
\text { extracellular } \\
\text { matrix-associated } \\
\text { proteins }\end{array}$ & $2.35 \mathrm{E}-04$ & $\begin{array}{c}\text { HBEGF } \mid \text { NRG1 } \mid \text { EREG } \mid \text { TGFA } \mid \text { CSF2 |VEGFC } \\
\mid \text { PLAU } \mid \text { COL13A1 }\end{array}$ & DM331 \\
\hline PID_TCR_CALCIUM_PATHWAY & $\begin{array}{l}\text { Calcium signaling } \\
\text { in the CD4+ TCR } \\
\text { pathway }\end{array}$ & $2.83 \mathrm{E}-04$ & CSF2|FOSL1|PTGS2 & DM331 \\
\hline NABA_MATRISOME_ASSOCIATED & $\begin{array}{l}\text { Ensemble of genes } \\
\text { encoding ECM- } \\
\text { associated } \\
\text { proteins including } \\
\text { ECM-affilaited } \\
\text { proteins, ECM } \\
\text { regulators and } \\
\text { secreted factors }\end{array}$ & $2.83 \mathrm{E}-04$ & $\begin{array}{c}\text { HBEGF | NRG1|EREG |TGFA |CSF2 |VEGFC } \\
\mid \text { PLAU }\end{array}$ & DM331 \\
\hline PID_ERBB4_PATHWAY & $\begin{array}{c}\text { ErbB4 signaling } \\
\text { events }\end{array}$ & 4.42E-04 & HBEGF|NRG1|EREG & DM331 \\
\hline $\begin{array}{c}\text { REACTOME_NUCLEAR_SIGNALIN } \\
\text { G_BY_ERBB4 }\end{array}$ & $\begin{array}{c}\text { Genes involved in } \\
\text { Nuclear signaling } \\
\text { by ERBB4 }\end{array}$ & $4.42 \mathrm{E}-04$ & HBEGF|NRG1|EREG & DM331 \\
\hline PID_AP1_PATHWAY & $\begin{array}{l}\text { AP-1 transcription } \\
\text { factor network }\end{array}$ & 1.07E-04 & IL8|FOSL1|EGR1|CCND1|GJA1|NR3C1 & SKMEL24 \\
\hline PID_NFAT_TFPATHWAY & $\begin{array}{l}\text { Calcineurin- } \\
\text { regulated NFAT- } \\
\text { dependent } \\
\text { transcription in } \\
\text { lymphocytes } \\
\end{array}$ & $2.25 \mathrm{E}-04$ & IL8|FOSL1|EGR1|PTGS2|EGR3 & SKMEL24 \\
\hline $\begin{array}{c}\text { KEGG_CYTOKINE_CYTOKINE_REC } \\
\text { EPTOR_INTERACTION }\end{array}$ & $\begin{array}{l}\text { Cytokine-cytokine } \\
\text { receptor } \\
\text { interaction }\end{array}$ & 7.99E-04 & $\begin{array}{c}\text { IL8|PDGFA|IL1B }|| L 1 A|C X C L 1| C C L 20 \mid \text { TN } \\
\text { FRSF11B|TNFRSF12A }\end{array}$ & SKMEL24 \\
\hline NABA_MATRISOME & $\begin{array}{c}\text { Ensemble of genes } \\
\text { encoding } \\
\text { extracellular } \\
\text { matrix and } \\
\text { extracellular } \\
\text { matrix-associated } \\
\text { proteins }\end{array}$ & $1.15 \mathrm{E}-03$ & $\begin{array}{c}\text { IL8|PDGFA |IL1B |IL1A|CXCL1|CCL20|SE } \\
\text { MA5A|SEMA4B |CLEC2B|SERPINB8|THB } \\
\text { S2|CTGF|ABI3BP|CRISPLD2 }\end{array}$ & SKMEL24 \\
\hline PID_FRA_PATHWAY & $\begin{array}{c}\text { Validated } \\
\text { transcriptional } \\
\text { targets of AP1 } \\
\text { family members } \\
\text { Fra1 and Fra2 }\end{array}$ & $1.34 \mathrm{E}-03$ & IL8|FOSL1|CCND1|GJA1 & SKMEL24 \\
\hline BIOCARTA_PPARA_PATHWAY & $\begin{array}{c}\text { Mechanism of } \\
\text { Gene Regulation } \\
\text { by Peroxisome } \\
\text { Proliferators via } \\
\text { PPARa(alpha) }\end{array}$ & $6.83 \mathrm{E}-03$ & PTGS2|PDGFA|NR2F1|CITED2 & SKMEL24 \\
\hline BIOCARTA_NTHI_PATHWAY & $\begin{array}{l}\text { NFkB activation by } \\
\text { Nontypeable } \\
\text { Hemophilus } \\
\text { influenzae }\end{array}$ & $1.02 \mathrm{E}-02$ & IL8|NR3C1||IL1B & SKMEL24 \\
\hline BIOCARTA_INFLAM_PATHWAY & $\begin{array}{l}\text { Cytokines and } \\
\text { Inflammatory } \\
\text { Response }\end{array}$ & $1.45 \mathrm{E}-02$ & IL8|PDGFA|IL1A & SKMEL24 \\
\hline NABA_MATRISOME_ASSOCIATED & $\begin{array}{l}\text { Ensemble of genes } \\
\text { encoding ECM- } \\
\text { associated } \\
\text { proteins including } \\
\text { ECM-affilaited }\end{array}$ & $1.45 \mathrm{E}-02$ & $\begin{array}{l}\text { IL8|PDGFA|IL1B|IL1A|CXCL1|CCL20|SE } \\
\text { MA5A|SEMA4B |CLEC2B |SERPINB8 }\end{array}$ & SKMEL24 \\
\hline
\end{tabular}




\begin{tabular}{|c|c|c|c|c|}
\hline & $\begin{array}{l}\text { proteins, ECM } \\
\text { regulators and } \\
\text { secreted factors }\end{array}$ & & & \\
\hline PID_REG_GR_PATHWAY & $\begin{array}{l}\text { Glucocorticoid } \\
\text { receptor } \\
\text { regulatory } \\
\text { network }\end{array}$ & $1.45 \mathrm{E}-02$ & IL8|EGR1|NR3C1|VIPR1 & SKMEL24 \\
\hline BIOCARTA_CELLCYCLE_PATHWAY & $\begin{array}{l}\text { Cyclins and Cell } \\
\text { Cycle Regulation }\end{array}$ & $4.62 \mathrm{E}-05$ & CDKN2A|CCND1|CCNA1|CDK6 & SKMEL28 \\
\hline PID_AP1_PATHWAY & $\begin{array}{l}\text { AP-1 transcription } \\
\text { factor network }\end{array}$ & $4.62 \mathrm{E}-05$ & CDKN2A|CCND1|FOSL1|FOS|EGR1 & SKMEL28 \\
\hline BIOCARTA_G1_PATHWAY & $\begin{array}{l}\text { Cell Cycle: G1/S } \\
\text { Check Point }\end{array}$ & $4.62 \mathrm{E}-05$ & CDKN2A|CCND1|CCNA1|CDK6 & SKMEL28 \\
\hline PID_CMYB_PATHWAY & $\begin{array}{c}\text { C-MYB } \\
\text { transcription } \\
\text { factor network }\end{array}$ & $7.12 \mathrm{E}-05$ & CDKN2A $\mid$ CCND1 $\mid$ CCNA1|CDK6|CEBPB & SKMEL28 \\
\hline PID_FRA_PATHWAY & $\begin{array}{c}\text { Validated } \\
\text { transcriptional } \\
\text { targets of AP1 } \\
\text { family members } \\
\text { Fra1 and Fra2 }\end{array}$ & $8.84 \mathrm{E}-05$ & CDKN2A|CCND1|FOSL1|PLAUR & SKMEL28 \\
\hline $\begin{array}{c}\text { SA_REG_CASCADE_OF_CYCLIN_E } \\
\text { XPR }\end{array}$ & $\begin{array}{c}\text { Expression of } \\
\text { cyclins regulates } \\
\text { progression } \\
\text { through the cell } \\
\text { cycle by activating } \\
\text { cyclin-dependent } \\
\text { kinases. }\end{array}$ & $2.22 \mathrm{E}-04$ & CDKN2A|CCND1|CCNA1 & SKMEL28 \\
\hline PID_TAP63_PATHWAY & $\begin{array}{c}\text { Validated } \\
\text { transcriptional } \\
\text { targets of TAp63 } \\
\text { isoforms }\end{array}$ & $2.96 \mathrm{E}-04$ & CDKN2A|GDF15|AEN|TFAP2C & SKMEL28 \\
\hline BIOCARTA_SPRY_PATHWAY & $\begin{array}{l}\text { Sprouty regulation } \\
\text { of tyrosine kinase } \\
\text { signals }\end{array}$ & $4.71 \mathrm{E}-04$ & SPRY2|SPRY1|SPRY4 & SKMEL28 \\
\hline PID_RB_1PATHWAY & $\begin{array}{l}\text { Regulation of } \\
\text { retinoblastoma } \\
\text { protein }\end{array}$ & 4.87E-04 & CDKN2A $\mid$ CCND1 $\mid$ CDK6|CEBPB & SKMEL28 \\
\hline $\begin{array}{c}\text { KEGG_JAK_STAT_SIGNALING_PA } \\
\text { THWAY }\end{array}$ & $\begin{array}{l}\text { Jak-STAT signaling } \\
\text { pathway }\end{array}$ & $5.92 \mathrm{E}-04$ & CCND1|SPRY2|SPRY1|SPRY4|SPRED2 & SKMEL28 \\
\hline
\end{tabular}

In both the RPPA and gene expression responses, we observe an intriguing

phenomenon. Responses of genes and proteins to single drugs appear to be

amplified by treatment with a secondary drug. This trend is apparent across all

times sampled by RPPA, as well as being apparent in the gene expression results,

regardless of the secondary drug compound. This indicates that the pathways

targeted by these drugs are contributing to this observed response, specifically the

combined inhibition of MAPK signaling with RTK driven signaling such as AKT or

ErbB signaling. Having observed the pronounced responses in the transcriptome 
and proteome, we sought to investigate if these responses were reflected at other levels of transcriptional regulation.

\subsubsection{PLX4720 treatment, lapatinib treatment, or the combination} treatment does not drive responses in DNA methylation

To interrogate other levels of transcriptional regulation, we turned to DNA methylation microarrays. As previously discussed, changes in DNA methylation often serve to silence tumor suppressors and have been observed in multiple cancers, including melanoma (de Araújo et al., 2015). To ascertain the effects of treatment on DNA methylation status, we profiled the basal methylome of our cell lines, and DM331 was subjected to the same panel of treatments as performed for gene expression. We were unable to observe any significant alterations in DNA methylation loci under any treatment conditions for DM331 (Figure 3.5). This indicates that MAPK pathway inhibition or RTK inhibition are insufficient to induce changes in DNA methylation at 8 hours. As with our other analyses of functional genomics data, we were curious to know whether DNA methylation status serves as a marker of sensitivity or synergy in our panel, as other studies had shown that DNA methylation is an effective profiling tool for patient outcome (de Araújo et al., 2015; Conway et al., 2011; Thomas et al., 2014). 


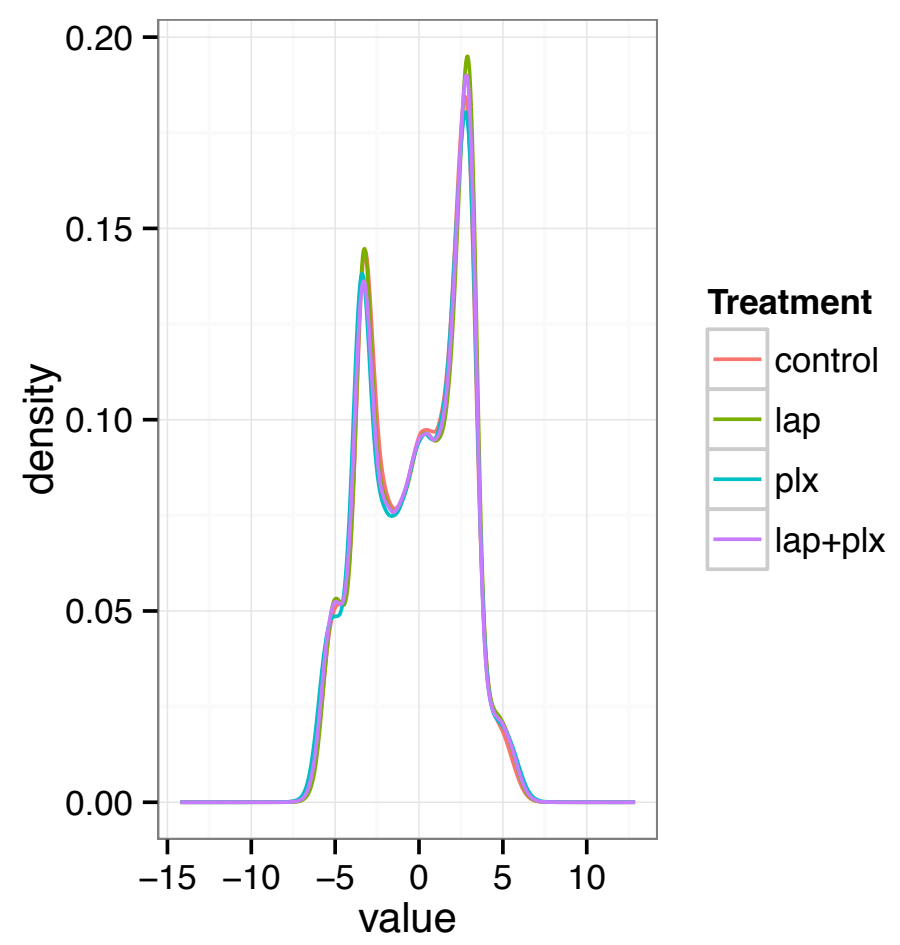

Figure 3.5 DNA methylation status of DM331 does not significantly change in response to single or combination treatment

DNA methylation was assayed in DM331 after 8 hours exposure to lapatinib (lap) alone, PLX4720 (plx) alone or the combination of the two drugs (lap+plx). DNA methylation beta values were normalized and logit transformed prior to a moderated t-test being applied to identify differentially methylated loci. No loci were found to be differentially methylated in response to treatment, and logit distributions (derived from $M$ values) were plotted to demonstrate the lack of a distribution shift. Samples were prepared by Devin Roller, in the Weber lab at the University of Virginia.

Observing no significant response in the DNA methylome of DM331 or SKMEL24 in response to treatment, we profiled all twelve BRAFV600E melanomas to determine if methylation status associated with any of our previously observed phenotypes. We began by performing a principal components analysis on the methylome of all twelve lines to determine if the basal methylation state could be used to distinguish lines that demonstrate synergy to the combination of PLX4720 and lapatinib. DNA methylation status does not appear to segregate lines based on their synergy status, as evidenced by the distribution of the synergistic lines in the 
PCA plot (Figure 3.6). We were astonished to observe that in another functional genomic profile, DM331 very closely matched the profile of A375. This indicates that if DM331 basal methylation is allowing for its more robust adaptive response, it is restricted to a small number of differentially methylated loci.

We observed that DM6 appears to contribute the most variation along the first principal component. It is the only line on the left side of the plot, indicating that its basal methylation state is extremely different from any of the other lines. We sought to determine the nature of this difference by plotting the distribution of beta values for all twelve cell lines (Figure 3.7). Here we are able to observe that DM6 is globally hypomethylated to the point of having almost no methylated loci. Loss of DNA methylation is often associated with invasive phenotypes. When we reexamined the exome of DM6, we do observe a mutation in DNMT3A, which could be preventing the maintenance of DNA methylation in this cell line. We wondered if removing DM6 because of its outlier nature with regards to its global hypomethylation could better refine our analysis. 


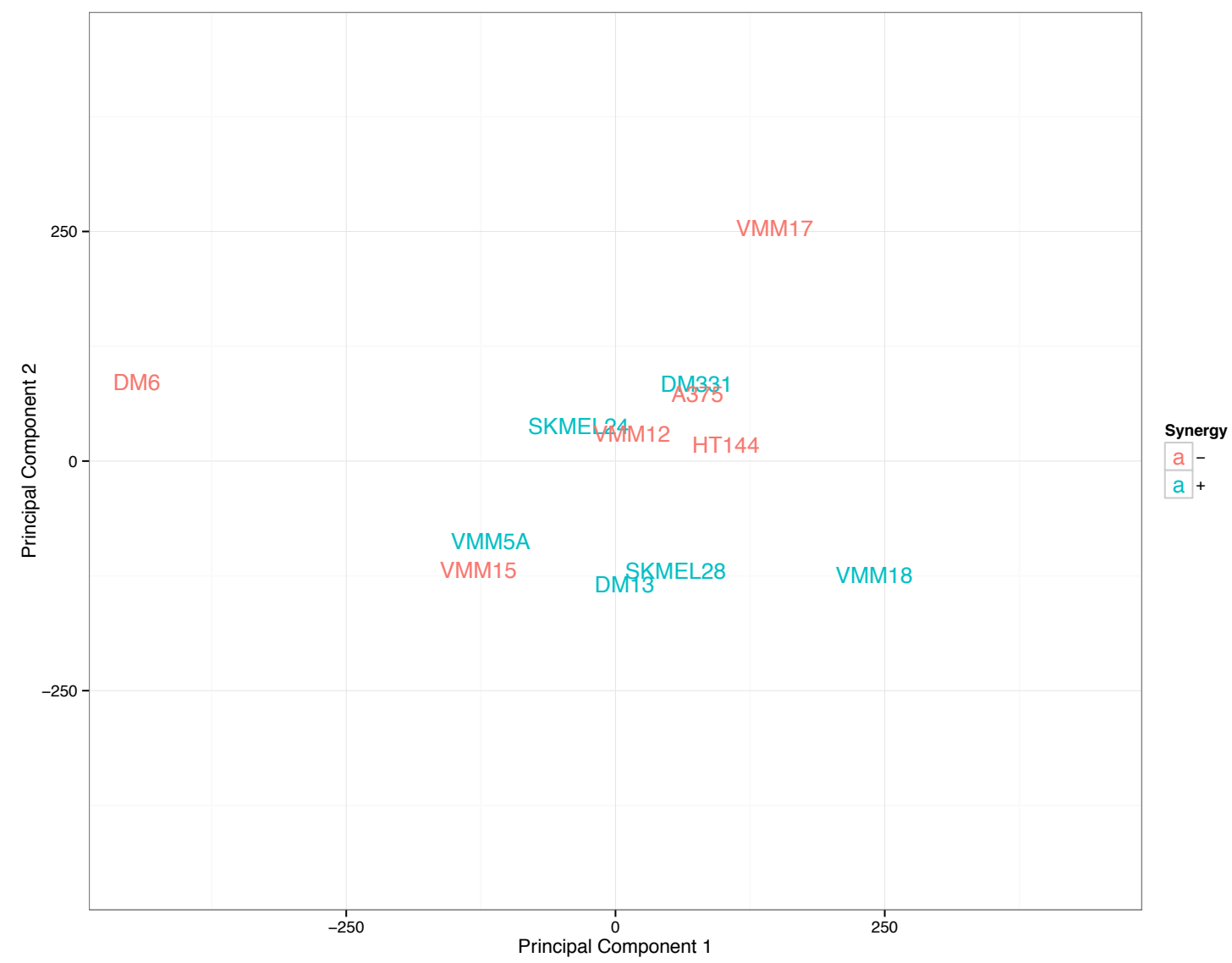

Figure 3.6 DM6 has a unique DNA methylation profile based on its separation in the principal component analysis

Principal component analysis of basal DNA methylation $M$ values across 12 melanoma cell lines. Lines that demonstrate synergy to the combination of PLX4720 and lapatinib are colored blue, and lines that do not show synergy are colored red. The DNA methylation state of DM6 is driving the vast amount of separation between the lines along the first principal component. As with the gene expression and exome profiling of the lines, DM331 and A375 have very few differences. Samples were prepared by Devin Roller, in the Weber lab at the University of Virginia. 


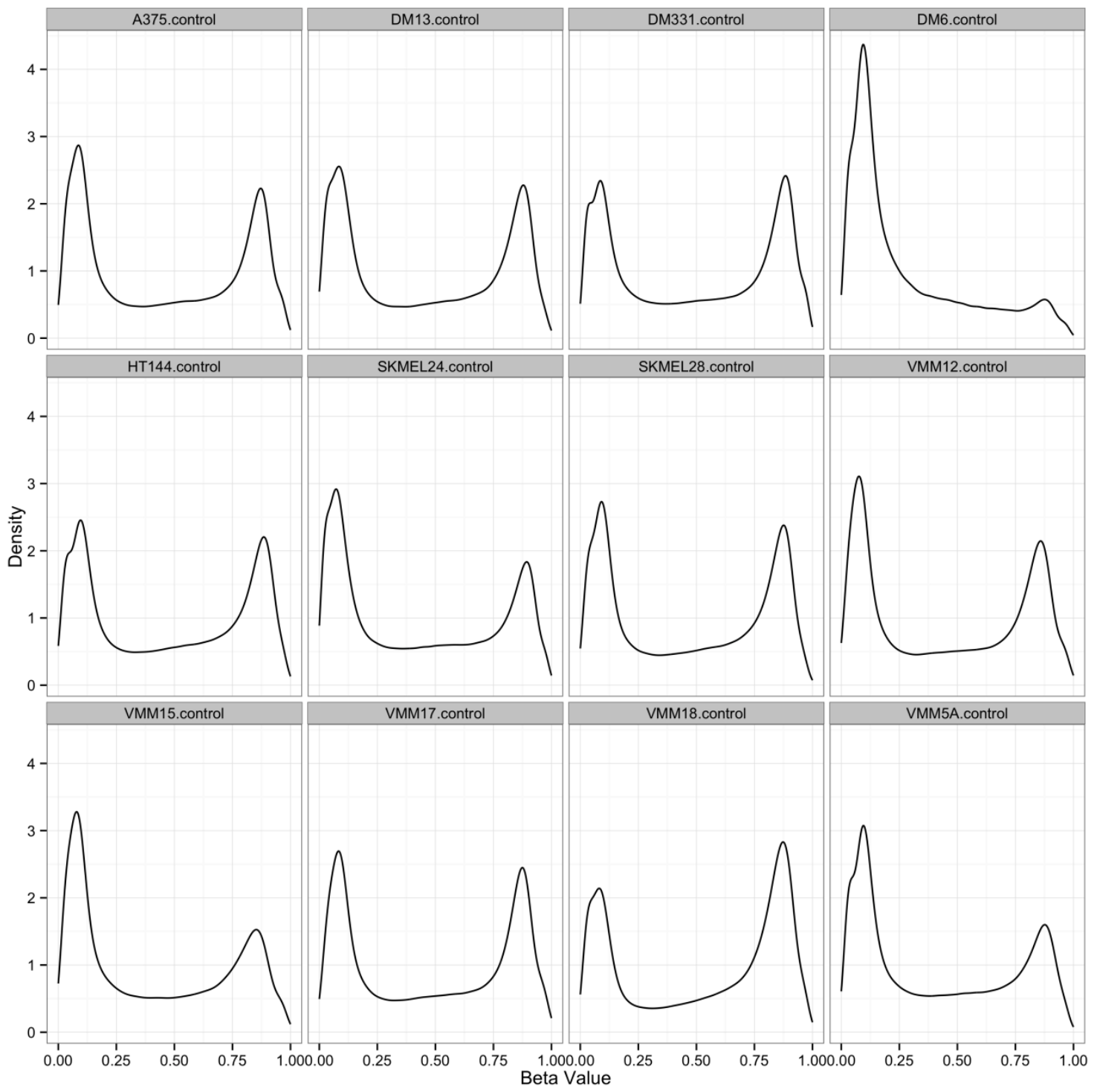

Figure 3.7 Separation of DM6 in PCA is attributed to its globally hypomethylated state

Beta values were obtained for all twelve melanoma cell lines and the density of the values were plotted for each cell line. DM6 appears to suffer from global hypomethylation which is most likely driving its strong separation along the first principal component. DM6 contains a nonsynonymous variant in DNMT3A, which could inhibit its ability to maintain methylated loci and explain its global hypomethylation. Samples were prepared by Devin Roller, in the Weber lab at the University of Virginia.

Following removing DM6 from the analysis, we still were unable to observe segregation of lines into phenotypic groups outlined elsewhere. We were intrigued

120 
that the DNA methylation analysis revealed relatively little about the nature of lines given the success we had had with other functional genomics assays. The DNA methylome does not appear to be altered by treatment with PLX4720, lapatinib, or the combination. The basal methylation profiles of the lines fail to stratify them into any distinct groups. Other studies have shown that loss of methylation at the EGFR locus is a known mechanism or resistance (Wang et al., 2014), so we sought to determine whether forced loss of methylation would enable sensitive lines to become resistant via RTK upregulation.

\subsubsection{Global hypomethylation induced by decitabine treatment sensitizes DM331 to lapatinib treatment \\ Literature has suggested that resistant melanomas show sensitivity to} decitabine treatment (Halaban et al., 2009) and that resistant melanomas leverage epigenetic switches to induce RTK signaling (Wang et al., 2014). Given the weak response of DM331 to PLX4720 treatment (Figure 3.8A), we sought to evaluate the effects of decitabine treatment on PLX4720 sensitivity. Using decitabine at sublethal doses we knocked down DNA methylation globally in DM331 and A375. We chose these two lines because of their aforementioned similarity across multiple genomic and functional genomic profiles, and the fact that they differ greatly in sensitivity to PLX4720 (Figure 3.8A). We began by determining a dose of decitabine treatment that inhibited DNMT activity, but did not cause any cytotoxic effects (Figure 3.8B). Once we obtained an appropriate dose - sublethal but still inhibitory of DNMT activity - we exposed DM331 and A375 to this dose of decitabine coupled with the high dose of PLX4720 with an escalating dose of lapatinib. Astoundingly, we observed that DM331 no longer demonstrated a synergistic effect from the 
combination of PLX4720 and lapatinib. A375 however, demonstrated no significant change in its response to combination treatment at any dose (Figure 3.8C and 3.8D). We became even more astonished when we observed that decitabine treatment does not change DM331 response to PLX4720 treatment (Figure 3.8E), but sensitized it to lapatinib treatment (Figure 3.8F).

A

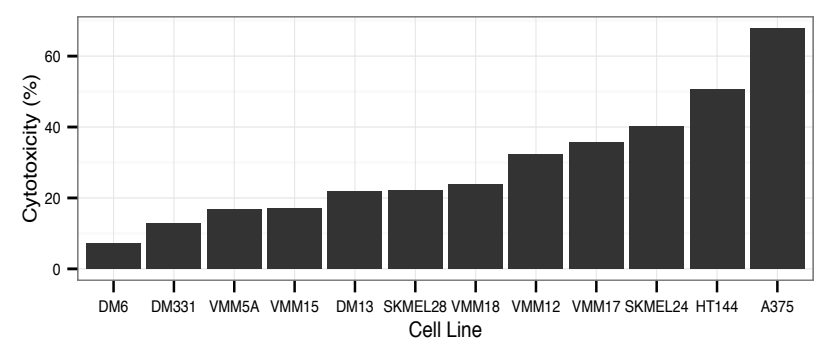

$B$

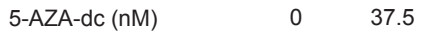

quantification of DMNT:

tubulin
C

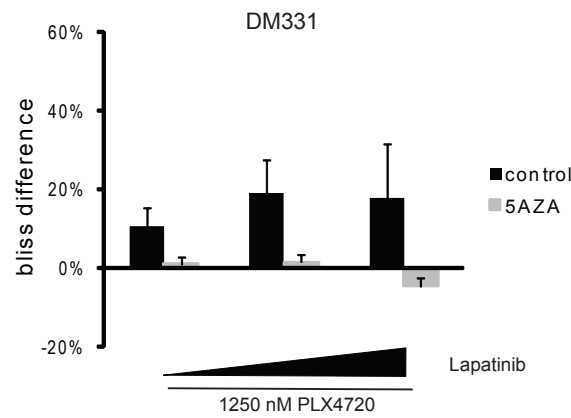

$E$

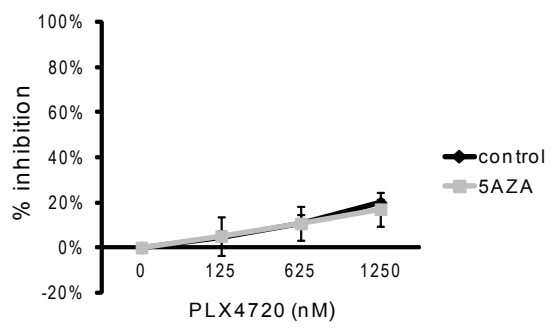

D $\quad 60 \%$

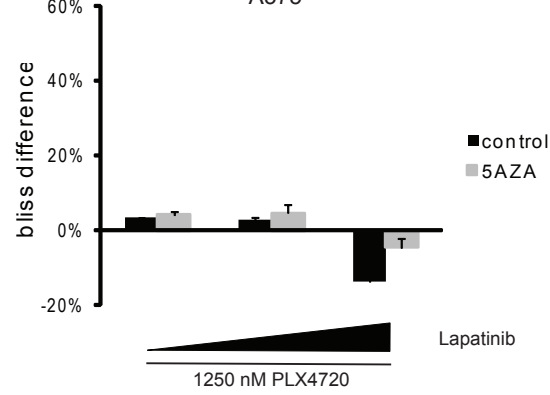

F

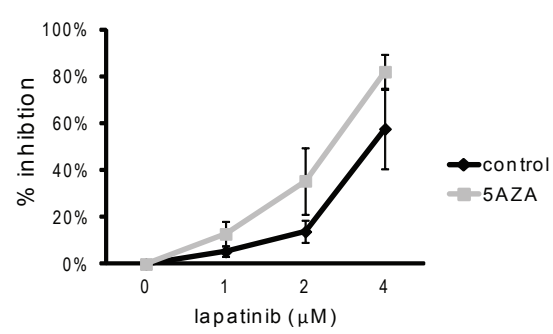

Figure 3.8 Global knockdown of DNA methylation sensitizes DM331 to lapatinib treatment

(A) Cell lines cytotoxic response 72 hours after treatment with 125nM PLX4720 were profiled. (B) DM331 cells were treated with 37.5nM 5-Aza-2'-deoxycytidine for 48 hours. Total protein was isolated and immunoblot analysis was conducted for DMNT and tubulin. Quantification of DMNT is shown. (C) DM331 or (D) A375 cells were treated with 5-Aza-2'-deoxycytidine( $37.5 \mathrm{nM}$ or $75 \mathrm{nM}$ ) every other day for 10 days. On day 7, 5-Aza-2'-deoxycytidine treated cells (gray bars) and control cells that have received no 5-Aza-2'-deoxycytidine treatment (black bars) were plated in 96 well plates and treated with $1250 \mathrm{nM}$ PLX4720 and lapatinib (1 $\mu \mathrm{M}, 2 \mu \mathrm{M}$, or $4 \mu \mathrm{M})$. Metabolic activity was read out using alamarBlue (n=3). Following 7 days of $37.5 \mathrm{nM}$ 
5-Aza-2'-deoxycytidine or control treatment DM331 were treated with (E) PLX4720 (125 nM, $625 \mathrm{nM}, 1250 \mathrm{nM}$ ), (F) lapatinib (1 $\mu \mathrm{M}, 2 \mu \mathrm{M}$, or $4 \mu \mathrm{M})$ or vehicle control for 3 days or and metabolic activity was read out using alamarBlue $(n=3)$. Samples and figure panels were prepared by Devin Roller, in the Weber lab at the University of Virginia.

Given the sensitization of DM331 to lapatinib treatment when exposed to sublethal doses of decitabine, we assayed the DNA methylation response to decitabine treatment and observed that both DM331 and A375 demonstrate global hypomethylation (Figure 3.9). We conclude that the level of hypomethylation is extremely significant; DM331 appears to be losing more methylated loci at a global level in response to decitabine treatment than A375. We wanted to determine the mechanism by which DM331 becomes more sensitive to lapatinib treatment, so we profiled its gene expression response to decitabine treatment. 


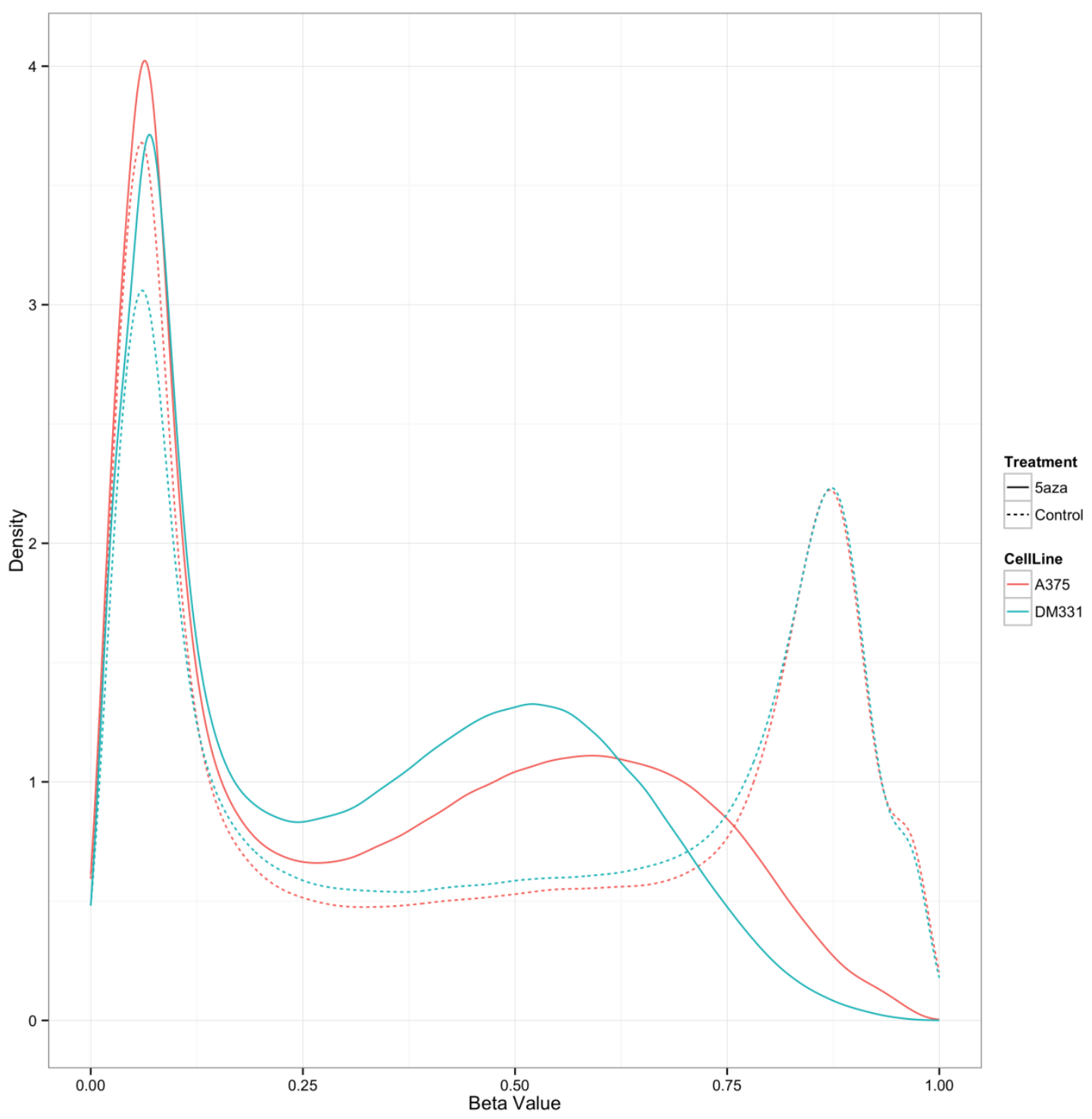

\section{Figure 3.9 Global knockdown of DNA methylation using decitabine is more robust in DM331 than $\mathbf{A 3 7 5}$}

Lines were treated with decitabine as described in Figure 3.8 or with vehicle control. After treatment, cells were harvested and DNA methylation was assayed using the Illumina 450K DNA methylation bead chip. Beta values were plotted for A375 (red) or DM331 (blue) after treatment with decitabine (5aza, solid line) or vehicle control (Control, dashed line). Samples were prepared by Devin Roller, in the Weber lab at the University of Virginia. 


\subsubsection{Decitabine treatment induces the same group of transcription factors}

that are induced by PLX4720 treatment in lines that respond synergistically to the combination of PLX4720 with lapatinib

We exposed DM331 and A375 to the same dose of decitabine treatment as in our cytotoxicity assays to determine the mechanism by which sublethal doses of decitabine were sensitizing DM331 to lapatinib treatment. We identified a number of differentially expressed genes in both lines, but also observed very few genes that showed significant response in both lines (Figure 3.10). We took the list of differentially expressed genes for each line through a pathway and transcription factor enrichment analysis to determine the broad changes that treatment with decitabine may be affecting. We observed an upregulation of genes associated with the same transcription factors that we saw controlling the ErbB signaling pathway in our other lines, indicating the decitabine treatment potentially induces the ErbB signaling response in a manner similar to that of PLX4720 treatment (Table 3.4). This suggests that the sensitivity to lapatinib induced by PLX4720 treatment is induced similarly by decitabine treatment. This result also may explain the observed loss of synergy; if the ErbB pathway is already induced by decitabine, then PLX4720 cannot effect these changes any further. Taken together, these observations imply that there is an upper limit to the adaptive response a cell line is able to utilize. DM331 does not end up being excessively responsive to repeated targeting of the ErbB signaling pathway. 

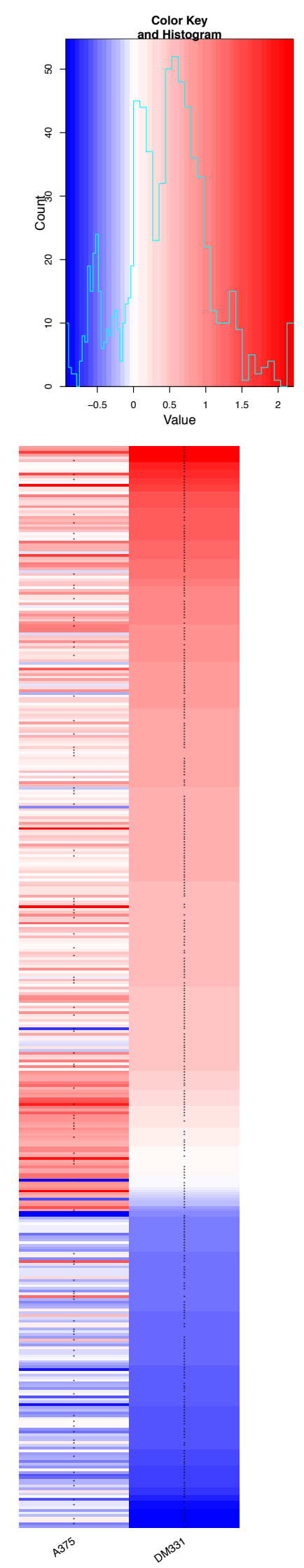


\section{Figure 3.10 Decitabine treatment in DM331 induces significant alterations to its gene expression profile}

Heat map of log2 fold changes induced by decitabine treatment in A375 and DM331. Fold changes are ordered by magnitude in DM331, with fold changes having a FDR or $1 \%$ or better being marked with “*”. Samples were prepared by Devin Roller, in the Weber lab at the University of Virginia.

\section{Table 3.4 MSigDB transcription factor enrichment results for genes differentially expressed in DM331 after treatment with decitabine.}

\begin{tabular}{|c|c|c|c|}
\hline $\begin{array}{c}\text { Gene Set } \\
\text { Name }\end{array}$ & Description & Enrichment FDR q-value & Significant genes in gene set \\
\hline $\begin{array}{l}\text { GGGAGG } \\
\text { RR_V\$M } \\
\text { AZ_Q6 }\end{array}$ & $\begin{array}{c}\text { Motif GGGAGGRR; } \\
\text { MAZ }\end{array}$ & $1.53 \mathrm{E}-12$ & $\begin{array}{l}\text { COL1A1,VGF,H3F3B,SERPINB2,CADM1,ID3,COL1A2,POU3F2,T } \\
\text { MEM88,SLCO2A1,HAPLN1,NFKBIA,P4HA1,S100A4,FLNC,HCFC } \\
\text { 1R1,CD68,FERMT3,IL24,PADI3,VASN,STAG1,JUP,RCOR2,KCNM } \\
\text { A1,CXXC5,SOX15,ERBB3,YARS,PKP4,LAMA5,DAGLA,MDK,EHB } \\
\text { P1,SLC39A11,PPM1A,UBR4,PNKD,AHNAK,PLAT,INHBE,CLIP3,V } \\
\text { AMP8,EFEMP2,CCNE1,RAB31,TAGLN,SCD,GMFG,ANKRD11,PR } \\
\text { R16,SELM,TRMT11,QPRT,PLAUR }\end{array}$ \\
\hline $\begin{array}{c}\text { TATAAA } \\
\text { V\$TATA } \\
\quad 01\end{array}$ & Motif TATAAA; TAF & $3.53 \mathrm{E}-12$ & $\begin{array}{c}\text { COL1A1,VGF,H3F3B,SERPINB2,CADM1,ID3,COL1A2,POU3F2,T } \\
\text { MEM88,SLCO2A1,HAPLN1,NFKBIA,P4HA1,S100A4,TUBA4A,CP } \\
\text { A4,KRT8,DCAKD,BMP5,S100A2,TFAP2A,LCP1,LAPTM5,CRABP2 } \\
\text {,SOX2,TNF,TNFSF10,GPC6,SERPINE1,CCL5,ICK,CD24,THBS2,IG } \\
\text { FBP4,HIST1H2BK,ACTG2,HIST1H2BD,VCAM1,NDUFA7,APCDD } \\
\text { 1L }\end{array}$ \\
\hline $\begin{array}{c}\text { TGANTC } \\
\text { A_V\$AP } \\
\text { 1_C }\end{array}$ & Motif TGANTCA; JUN & $1.73 \mathrm{E}-11$ & $\begin{array}{c}\text { COL1A1,VGF,H3F3B,SERPINB2,FLNC,HCFC1R1,CD68,FERMT3,I } \\
\text { L24,PADI3,VASN,TUBA4A,CPA4,KRT8,DCAKD,BMP5,S100A2, E } \\
\text { NO3,NRIP3,LAMC2,SFN,CALB2,IL6,RCAN1,CSPG4,PDGFRB,TFB } \\
\text { 1M,COL7A1,MMP9,HCLS1,ISG20,PLAU,C19orf33,F3,MMP1,T } \\
\text { M4SF19 }\end{array}$ \\
\hline $\begin{array}{l}\text { CAGGTG } \\
\text { V\$E12_- } \\
\text { Q6 }\end{array}$ & Motif CAGGTG; TCF3 & $5.22 \mathrm{E}-11$ & $\begin{array}{c}\text { COL1A1,VGF,CADM1,ID3,COL1A2,POU3F2,TMEM88,SLCO2A1, } \\
\text { FLNC,STAG1,JUP,RCOR2,KCNMA1,CXXC5,SOX15,ERBB3,YARS, } \\
\text { PKP4,LAMA5,DAGLA,MDK,TUBA4A,CPA4,KRT8,TFAP2A,LCP1,L } \\
\text { APTM5,CRABP2,ENO3,NRIP3,LAMC2,SFN,HIF1A,SPRY2,DPYSL } \\
\text { 3,F2RL1,DEF6,GAD1,SKP2,CLDN7,PITX1,MFNG,ATF5,CHCHD3, } \\
\text { LTB,OLFML2A,C1QTNF1,RAC2,FAM162A,RBM47,SPINT2,POD } \\
\text { XL,LRIG1,SCG5 }\end{array}$ \\
\hline $\begin{array}{l}\text { AACTTT_- } \\
\text { UNKNO } \\
\text { WN }\end{array}$ & $\begin{array}{c}\text { Motif AACTTT; Gene } \\
\text { unknown }\end{array}$ & $9.19 \mathrm{E}-10$ & $\begin{array}{c}\text { CADM1,ID3,COL1A2,POU3F2,HAPLN1,NFKBIA,HCFC1R1,STAG } \\
\text { 1,JUP,RCOR2,KCNMA1,CXXC5,EHBP1,SLC39A11,PPM1A,UBR4, } \\
\text { DCAKD,BMP5,TFAP2A,SOX2,TNF, TNFSF10,GPC6,ENO3,CALB2, } \\
\text { HIF1A,SPRY2,DPYSL3,F2RL1,KYNU,EFNB2,SPHK1,FLRT3,SAP30, } \\
\text { DACT1,SLC6A15,PCK2,LRP8,TMEM47,RILPL1,PTGS2,CAPS,SOX } \\
\text { 18,PSAT1 }\end{array}$ \\
\hline $\begin{array}{l}\text { GGGTGG } \\
\text { RR_V\$P } \\
\text { AX4_03 }\end{array}$ & $\begin{array}{c}\text { Motif GGGTGGRR; } \\
\text { PAX4 }\end{array}$ & $9.54 \mathrm{E}-09$ & $\begin{array}{l}\text { COL1A1,CADM1,ID3,TMEM88,HAPLN1,FLNC,HCFC1R1,STAG1, } \\
\text { JUP,RCOR2,SOX15,PNKD,AHNAK,PLAT,INHBE,CLIP3,TUBA4A,D } \\
\text { CAKD,SERPINE1,NRIP3,IL6,HIF1A,SPRY2,KYNU,EFNB2,SPHK1,D } \\
\text { DIT4,COL6A3,QPCT,ARHGDIB,MAP1LC3A,HK2,FOLR1,HYAL1 }\end{array}$ \\
\hline $\begin{array}{l}\text { TGGAAA } \\
\text { _V\$NFAT } \\
\text { _Q4_01 }\end{array}$ & Motif TGGAAA; NFAT & $9.54 \mathrm{E}-09$ & $\begin{array}{l}\text { VGF,CADM1,COL1A2,HAPLN1,NFKBIA,FLNC,HCFC1R1,CD68,ST } \\
\text { AG1,KCNMA1,ERBB3,YARS,PKP4,EHBP1,PNKD,VAMP8,BMP5, } \\
\text { SOX2,TNF,TNFSF10,CCL5,IL6,RCAN1,CSPG4,HIF1A,SPRY2,DEF6 } \\
\text {,GAD1,SKP2,KYNU,FLRT3,FAM107B,TMEM97,CCL2,ANGPTL2, } \\
\text { PLAC1,EFHD1,HLA-B,TNFRSF1B,ISG15,IL23R,PYGB }\end{array}$ \\
\hline $\begin{array}{l}\text { TTGTTT_- } \\
\text { V\$FOXO } \\
4 \_01\end{array}$ & Motif TTGTTT; MLLT7 & $3.11 \mathrm{E}-07$ & $\begin{array}{l}\text { COL1A1,H3F3B,CADM1,ID3,COL1A2,TMEM88,HAPLN1,CD68,S } \\
\text { TAG1,JUP,CXXC5,ERBB3,EHBP1,PNKD,BMP5,TFAP2A,SOX2,ICK } \\
\text {,CD24,LAMC2,PDGFRB,TFB1M,HIF1A,CLDN7,PITX1,FLRT3,SAP } \\
\text { 30,DACT1,DDIT4,FAM107B,TMEM97,EVL,SLC12A8,BIK,LRRC1 } \\
\text { 7,TMPRSS15,HMGB2,INSIG1,HSPA2,EXOC4,TSEN15 }\end{array}$ \\
\hline $\begin{array}{l}\text { GGGCGG } \\
\text { R_V\$SP1 } \\
\quad \text { _Q6 }\end{array}$ & Motif GGGCGGR; SP1 & $3.11 \mathrm{E}-07$ & $\begin{array}{l}\text { COL1A1,VGF,CADM1,COL1A2,POU3F2,TMEM88,NFKBIA,P4HA } \\
\text { 1,HCFC1R1,FERMT3,STAG1,JUP,KCNMA1,CXXC5,ERBB3,YARS, } \\
\text { LAMA5,DAGLA,EHBP1,SLC39A11,PPM1A,PNKD,AHNAK,PLAT,E } \\
\text { FEMP2,CCNE1,RAB31,SOX2,TNF,GPC6,ENO3,RCAN1,CSPG4,C } \\
\text { OL7A1,DEF6,GAD1,CLDN7,MFNG,ATF5,CHCHD3,SAP30,SLC6A }\end{array}$ \\
\hline
\end{tabular}




\begin{tabular}{|c|c|c|c|}
\hline & & & $\begin{array}{l}\text { 15,PCK2,LYPD3,MTHFD1L,CLDN11,CLIC3,MAEL,ASNS,SUSD2,K } \\
\text { RT7 }\end{array}$ \\
\hline $\begin{array}{l}\text { RYTTCCT } \\
\text { G_V\$ETS } \\
\text { 2_B }\end{array}$ & $\begin{array}{l}\text { Motif RYTTCCTG; } \\
\text { ETS2 }\end{array}$ & $4.34 \mathrm{E}-07$ & $\begin{array}{c}\text { VGF,ID3,HAPLN1,FERMT3,IL24,YARS,SLC39A11,VAMP8,LCP1,T } \\
\text { HBS2,IGFBP4,PDGFRB,TFB1M,MMP9,HCLS1,DEF6,LTB,DDIT4, } \\
\text { COL6A3,QPCT,ARHGDIB,CCL2,EVL,GTSF1,CSF2,ACSL5,KLRC2, } \\
\text { NFRSF6B }\end{array}$ \\
\hline
\end{tabular}

\subsection{DISCUSSION}

\subsubsection{High throughput screening of combinations reveals pathway dependencies for melanoma}

In our panel of BRAF ${ }^{\mathrm{V} 600 \mathrm{E}}$ melanoma we identified a number of combinations that demonstrated synergy across a panel of cell lines. The majority of the synergistic combinations we identified were dependent on inhibiting the MAPK and PI3K signaling pathway. Previously, other groups have identified the significance of these pathways (Carnero et al., 2008; Chandarlapaty et al., 2011; Kugel et al., 2014; McCubrey et al., 2006) and their reciprocity under conditions of inhibition of one pathway or the other. Here, we show that synergistic combinations are strongly dependent on inhibiting both pathways simultaneously. We are especially intrigued by the dependence of melanomas on RTK signaling previously observed by others (Abel et al., 2013; Held et al., 2013; Molhoek et al., 2011; Nazarian et al., 2010). Inhibiting specific RTKs appeared to be a very successful strategy for generating combinations, as evidenced by lapatinib, masitinib, and PI-103 all showing synergy. RTKs can induce MAPK and PI3K signaling depending on the context of the activation (McCubrey et al., 2006), and the observed synergy from targeting PDK1, a protein that sits above both pathways (Scortegagna et al., 2015) suggests that combinations which target RTK signaling in conjunction with MAPK signaling yield effective and consistent synergies. 


\subsubsection{Targeting proximal pathways with drug combinations induces potentiative responses in the proteome and transcriptome}

Given the evidence that combining RTK inhibition with MAPK pathway inhibition often yields synergies, we utilized RPPA to examine the effects on the proteome in response to treatment. For our analysis of treatment response, we selected two lines heterozygous for the BRAF V600E allele to study in depth, and explicitly looked at their responses in the MAPK signaling pathway and the PI3K pathway. We see many potentiative responses when comparing combination responses to single drug responses. We also observed that PLX4720 has a pronounced effect on AKT phosphorylation, what appears to be an adaptive response. As expected, based on the work presented in Chapter 2, under treatment with the combination, we observe the ablation of this purported adaptive response. Taken together, these results indicate that proximal drug targets tend to induce potentiative responses. Investigating the response trends further leads us to a gene expression analysis.

In our transcriptional analysis of the responses of the BRAFV600E melanomas to PLX4720 treatment with lapatinib and masitinib, we again observed that the targeting of proximal pathways elicits a potentiative pattern in the gene expression profiles. We were able to further investigate lines that respond to the combination synergistically and lines that do not. SKMEL24 and SKMEL28 both show excessive numbers of genes that demonstrated potentiative responses to combination treatment. We also observed that most significant responses were limited to each line individually. It appeared that every line had a unique set of responses that were 
induced by treatment. Though most lines showed similar patterns of responses in the genes, these changes were not typically significant for multiple lines.

Unexpectedly, A375 showed ablation of PLX4720 treatment effects in the transcriptome when treated with the combination. Given the small number of lines we screened, it is unclear whether this potentiation effect should be more or less prevalent given the treatment. We also were able to determine, following the exome analysis approach we present in Chapter 2, that our transcriptome treatment course included four lines homozygous for the BRAF V600E allele. It is telling that SKMEL24, the only line heterozygous for this allele, was also the most responsive line (based on the number of genes differentially expressed). It is unclear as to whether the zygosity of the allele is strongly influencing the transcriptional responses we observed. The changes observed in response to treatment in the proteome and transcriptome led us to investigate whether the DNA methylome was also being affected by treatment.

\subsubsection{Combination of PLX4720 and lapatinib induces no appreciable changes in the methylome \\ We profiled the methylome in DM331 and SKMEL24 after treatment with the} combination, but observed no significant changes in the methylation levels in any of their loci. This would seem to indicate that the methylome is not driving the adaptive response, nor is it the mechanism of synergy. However, we sought to determine if the methylome was a predictor of sensitivity to PLX4720.

When we profiled the methylomes of the twelve BRAFV600E melanomas, we observed the strong contribution of DM6 to the separation along the first principal 
component. We explored this further and observed that DM6 is globally hypomethylated, and were able to identify a variant in DMNT3A, a DNA methyl transferase. This variant could explain the inability of DM6 to maintain its methylation. Removing DM6 from the methylation analysis did not serve to capture any groups of lines that showed phenotypes we had identified in Chapter 2. The lack of change in methylation status after treatment with our combination as well as the lack of separation of our lines by methylome status perplexed us, as others had shown that loss of methylation at particular loci constitutes a mechanism of resistance to MAPK pathway inhibition (Wang et al., 2014). Given the diversity in our panel of cell lines, we wondered whether forced loss of methylation could recapitulate the resistance mechanism, and if our combination could overcome the resistance.

The observed similarities between DM331 and A375 at the transcriptome and exome and differnces in sensitivity to PLX4720 treatment made these two lines prime candidates for the forced methylation loss experiment. In the transcriptome and exome profiles, these two lines were always among the most similar. We again observed their similarities in the profiling of the methylome. We became interested in isolating the mechanisms by which DM331 exhibits its strong resistance to PLX4720 treatment and its synergistic response to the combination, whereas A375 - nearly identical to DM331 in exome, transcriptome, and methylome - is among the most sensitive to PLX4720 treatment, and does not demonstrate any appreciable increase in its response to the combination. These two lines demonstrate the 
significance that every line can respond differently, even when their functional genomic profiles are extremely similar.

We examined the effects of global demethylation in DM331 and A375 and were astonished to observe that DM331 became sensitive to lapatinib treatment under these conditions. A375 showed no response to the same protocol. The sensitization of DM331 to lapatinib by decitabine treatment prevented DM331 from demonstrating synergy in the presence of the combination. Most interestingly, treatment with decitabine induces the same set of transcription factors that we observed being induced by PLX4720 treatment. It is possible that there is a limit of adaptations that cells can undergo. Because decitabine induces an adaptive response similar to PLX4720, it might not be possible for DM331 to adapt again. The other possibility is that methylation is the mechanism of adaptation, however we do not observe any appreciable changes in methylation in response to any of the treatments. We note that the limit of this analysis is that we assayed one cell line at one time point, 8 hours after treatment with PLX4720, lapatinib, or the combination. 


\subsection{METHODS}

\subsubsection{Cell culture and cytotoxicity data generation}

\subsubsection{Melanoma cell culture}

SKMEL2, SKMEL24, SKMEL28 cells (American Type Culture Collection), A375, HT144, VMM5A, VMM39, VMM17, VMM18, VMM12, VMM15, DM13, DM6

DM122, DM331 (kind gift from Dr. Craig Slingluff, University of Virginia, Charlottesville, VA), and SLM2 (kind gift from Dr. Angela Zarling, University of Virginia) were propagated in RPMI-1640 (Invitrogen) supplemented with 5\% or 10\% FBS (Gemini Bio-Products). All melanoma cultures were maintained in a humidified chamber at $37^{\circ} \mathrm{C}$ with $5 \% \mathrm{CO}_{2}$. Inhibitors were obtained from the following sources: Lapatinib (LC Laboratories), 5-Aza-2'-deoxycytidine (decitabine) (Sigma). PLX4720 was a gift from Plexxikon.

\subsubsection{Cytotoxicity assays}

Four hours after being plated in 96-well plates, cells were treated with inhibitors or vehicle control in phenol red-free RPMI-1640 (Invitrogen) without FBS and incubated for 3 days at $37^{\circ} \mathrm{C}$. alamarBlue (Invitrogen) was added to wells and incubated for 4 hours at $37^{\circ} \mathrm{C}$. Fluorescence was measured at $560 \mathrm{~nm}$ excitation/590 nm emission on a Synergy 2 plate reader (BioTek Instruments). Mean results and SE were calculated for triplicate samples. 


\subsubsection{Generation of transcriptome, proteome, DNA methylome and exome data}

\subsubsection{Gene array}

Cells were plated and incubated overnight before being treated, in duplicate, with inhibitors or vehicle control in phenol red-free RPMI-1640 without FBS for 8 hours at $37^{\circ} \mathrm{C}$. Cells were placed on ice and rinsed with cold $1 \times$ PBS. Cells were collected and RNA was isolated using the Qiashredder (Qiagen) and RNeasy Mini Kit (Qiagen). RNA was quantified on the NanoDrop 2000 spectrophotometer (Thermo Scientific) and RNA quality was inspected on a 1\% agarose gel. Biotin labeled RNA was hybridized to Illumina 3'IVT human HT-12 BeadChip arrays.

\subsubsection{Methylation array}

Control cells were grown to $80 \%$ confluence, trypsinized, collected in a $15 \mathrm{ml}$ conical tube, and centrifuged to pellet. Cell pellet was washed twice in PBS, centrifuged, and stored at $-80^{\circ} \mathrm{C}$. DNA was isolated from cells using the DNeasy Blood and Tissue Kit (Qiagen, Valencia, CA) and profiled using the Illumina Methylation 450K array.

\subsubsection{Reverse Phase Protein Array}

Cells were plated in p60 dishes and incubated overnight to allow the cells to adhere before being treated with inhibitors or vehicle control in phenol red-free RPMI Medium 1640 without fetal bovine serum for 1, 8, or 24 hours. Cells were washed and lysed in 1:1 2x Sample Buffer:Tissue Extraction Reagent (T-PER) (Life Technologies). Following lysis the samples were sonicated and centrifuged to clear. Samples were diluted to requested concentration and sent to the lab of Emanuel (Chip) Petricoin at George Mason University for Reverse Phase Array Analysis. 


\subsubsection{Statistical Analysis of Functional Genomics Data}

Illumina microarray data was variance stabilized transformed (Federici et al., 2013; Improta et al., 2011) using the lumi Bioconductor package in R (Du et al., 2008). Significantly varying genes and cell lines were clustered using the R package pvclust (Suzuki and Shimodaira, 2006) with the Pearson correlation distance measure and average linkage. We identified genes whose expression was significantly altered for each cell lines in response to treatment using limma to perform moderated t-tests and applying a 1\% FDR cutoff. Illumina DNA methylation array data underwent normalization using SWAN and was logit transformed using minfi as described here (Aryee et al., 2014). A moderated t-test was performed as above to determine differentially methylated loci in response to treatment. Normalized $\log 2$ reverse phase protein array (RPPA) data was generated using methods described in (Improta et al., 2011). We performed a moderated t-test analysis described above (for gene expression data) and found a number of differentially phosphorylated sites at a FDR of $1 \%$.

\subsubsection{Pathway Enrichment Analysis}

Gene sets found to be significant underwent pathway enrichment analysis via Pathway Express and Gene Set Enrichment Analysis (GSEA) using MSigDB datasets. Significant gene sets and pathways were identified as having a FDR enrichment value of 0.05 or less for both tools.

\subsection{ACKNOWLEDGMENTS}

This chapter was adapted from (Roller et al., 2016) and (Roller et al., 2012). 


\section{Combination therapy in melanoma and B cell malignancies \\ demonstrates significant ability to overcome adaptive}

\section{survival signaling responses}

\subsection{ABSTRACT}

Combination therapy is able to block adaptive survival responses induced by single drug therapy in BRAFV600E melanoma. Here, we show that combination therapy extends to other preclinical models of melanoma, and is effective at blocking the adaptive response in B-cell malignancies induced by single drug therapy using both in vitro and ex vivo models. We also observe that combinations are not only able to induce amplification of single drug responses (potentiative), but are also capable of inducing novel responses at both the gene and potentially the pathway level (emergent). We show that the combination of sorafenib and diclofenac is an effective therapy for the treatment of multiple types of melanoma, and the efficacy of this combination is agnostic with respect to genetic drivers. We also observe that there exist a panel of combinations that are effective for in vitro models of mantle cell lymphoma and the synergistic response is maintained in ex vivo models of mantle cell lymphoma and chronic lymphocytic leukemia.

\subsection{INTRODUCTION}

As discussed previously, melanoma recurrence occurs in roughly half of all patients during treatment (Chapman et al., 2011). In chapter 2, we identified an adaptive response induced by PLX4720 treatment in resistant cell lines that 
leverages reactivation of the MAPK pathway through RTK signaling. In chapter 3, we showed that the adaptive response is effectively blocked by the inhibition of RTK signaling using lapatinib or masitinib, which shut down the reactivation of the MAPK pathway. We observed that the combination of these compounds elicited a potentiative response, indicating that for the combination PLX4720 with either lapatinib or masitinib, the second drug amplifies the effects of the first. This suggests that some cases of resistance involving reactivation of the pathway may be overcome through the increased inhibition of the pathway by using a secondary drug that targets a different member of the pathway.

Combination therapy has shown moderate success in melanoma (Chapman et al., 2014), however, much like single drug targeted therapy, responses to combination therapy have thus far not been durable (Fattore et al., 2015). In Chapter 3, we demonstrated the effectiveness of a screening strategy in BRAFV600E melanoma for identifying synergistic combinations. Therefore, we sought to determine the efficacy of our screening strategy in other types of melanoma, not driven by BRAFV600E. We were able to determine that the combination of sorafenib and diclofenac elicited a synergistic response across multiple types of melanoma (Roller et al., 2012). Sorafenib, similar to PLX4720, will inhibit BRaf, however, it is not as selective as PLX4720 and will target multiple kinases (Keating and Santoro, 2009). Diclofenac is a non-steroidal anti-inflammatory drug (NSAID) which inhibits cyclooxygenases (Dastidar et al., 2000). The efficacy of the combination of sorafenib and diclofenac did not correlate with RAF or RAS mutation status. Additionally, we observe that contrary to the potentiative response observed for PLX4720 and RTK 
inhibition, application of sorafenib with diclofenac in vitro yielded an emergent response in gene expression profiling. Significant levels of differential expression for some genes were only observed when both drugs were present, rather than the second drug amplifying the response of the first drug.

Lastly, we sought to apply our screening strategy in other cancer models available to us. Focusing on the B-cell malignancies, mantle cell lymphoma (MCL) and chronic lymphocytic leukemia (CLL), we were able to apply our screening strategy to identify a number of combinations found to be effective in both in vitro and ex vivo models. As with the combinations in melanoma, combinations for B-cell malignancies showed both potentiative and emergent responses. Research by others into combinatorial drug screening is focusing more on this phenomenon. In a study dealing with treatment of lines with combination therapy and the interactions between single and combination treatments (Cappuccio et al., 2015), the authors postulate that there are a limited number of patterns of response to single and combination treatments, and argue for the idea of positive synergy, and emergent positive synergy, whose definitions match our interpretations of potentiative and emergent responses.

As discussed in Chapter 1, CLL and MCL have poor prognoses, similar to melanoma, as well as being driven by the activation of a single growth pathway in a substantial portion of the patient population (Herman et al., 2011; Pérez-Galán et al., 2011). In melanoma, the aberrantly activated pathway is the MAPK pathway, in Bcell malignancies, it is the BCR pathway (Byrd et al., 2013; Pérez-Galán et al., 2011; Swerdlow and Williams, 2002; Wang et al., 2013). In addition to the parallels of 
cancer prognosis and propagation, melanoma and CLL and MCL also have potent targeted therapies that have been used to great effect in the patient population. Ibrutinib, a targeted therapy that inhibits Bruton's Tyrosine Kinase (BTK), a component of the BCR signaling pathway, shows overall response rate of $68 \%$ in patients with relapsed or refractory MCL, and $83 \%$ in patients with relapsed or refractory CLL (Axelrod et al., 2014a). As with melanoma, single agent therapy is not durable for CLL and MCL, as it induces adaptive responses, however, drug combinations that block adaptive signaling responses may increase both progression-free survival and overall survival in MCL (Liu et al., 2012; Pott et al., 2010).

Following the screening strategy utilized effectively in melanoma, the Weber lab was able to identify a number of drugs that when combined elicit a synergistic response in preclinical models of CLL and MCL (Axelrod et al., 2014a). Ibrutinib combined with ABT-199, an inhibitor of BCL2; or carfilzomib, or bortezomib, both of which are proteasome inhibitors, showed cytotoxic synergy. The combination of ibrutinib with ABT-199 presented the most striking results, showing rapid induction of apoptosis, and was characterized more than the other combinations. In addition the combination was found to be effective across ex vivo models derived from patients resistant to ibrutinib treatment, indicating its potential as a clinical treatment. 


\subsection{RESULTS}

\subsubsection{Sorafenib and diclofenac yield synergistic responses in melanoma regardless of primary driver mutations}

A synthetic lethal drug screen was performed with a panel of melanoma to include BRAFV600E, NRASQ61R, and BRAFWT /NRASWT melanomas. Given the multitude of driver mutations in melanoma (Hodis et al., 2012), we sought to determine if there were combinations of drugs that would be effective regardless of the genetic background, but still in the context of activated MAPK pathway. In this screen, we were able to identify the combination of sorafenib and diclofenac as a synergistic combination in cell lines of all three varieties, BRAFV600E, NRASQ61R, and

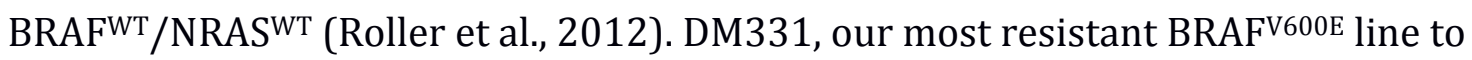
PLX4720 treatment, SLM2, a BRAFWT/NRASWT melanoma, and VMM39, a NRASQ61R melanoma all showed synergistic responses to this combination. The combination of sorafenib and diclofenac was the most effective across multiple lines. Even more astounding, was the combination showed synergy regardless of the driver mutation present in the melanoma.

We next wanted to determine how sorafenib and diclofenac induced synergy in our panel, and evaluate the similarity of the responses across different drivers. Using microarrays, we assayed the gene expression of two lines, VMM39 and SLM2 to determine how the treatment of sorafenib alone, diclofenac alone, and the combination were altering the transcriptional profiles of the lines (Figure 4.1). We observed that there were four types of responses; genes downregulated by the combination in VMM39, but weakly in SLM2; genes downregulated in both VMM39 
and SLM2; genes that have varied expression profiles between VMM39 and SLM2; and genes that are upregulated by the combination in VMM39 and SLM2. We wanted to determine what pathways these changes in gene expression might be associated with, so we performed a gene ontology enrichment analysis. We noted that a number of genes were associated with survival-related GO terms. Taken together, these observations suggest that the combination of sorafenib and diclofenac induces synergistic cytotoxicity at the gene expression level by affecting genes associated with survival. We observed that the responses that we were inducing across all three lines involved the induction of genes associated with GO terms of apoptosis and the negative regulation of survival. Unlike the screen discussed previously, sorafenib and diclofenac are not very specific targeted therapies. We wanted to ascertain the molecular targets of the drugs in this context, so we sought to identify more specific targeted compounds that would phenocopy the combination of sorafenib and diclofenac. Through a series of drug substitutions, we were able to determine that sorafenib was acting at least in part as a Raf/MAP Kinase inhibitor, and diclofenac was primarily acting as a Cox2 inhibitor (Roller et al., 2012). 


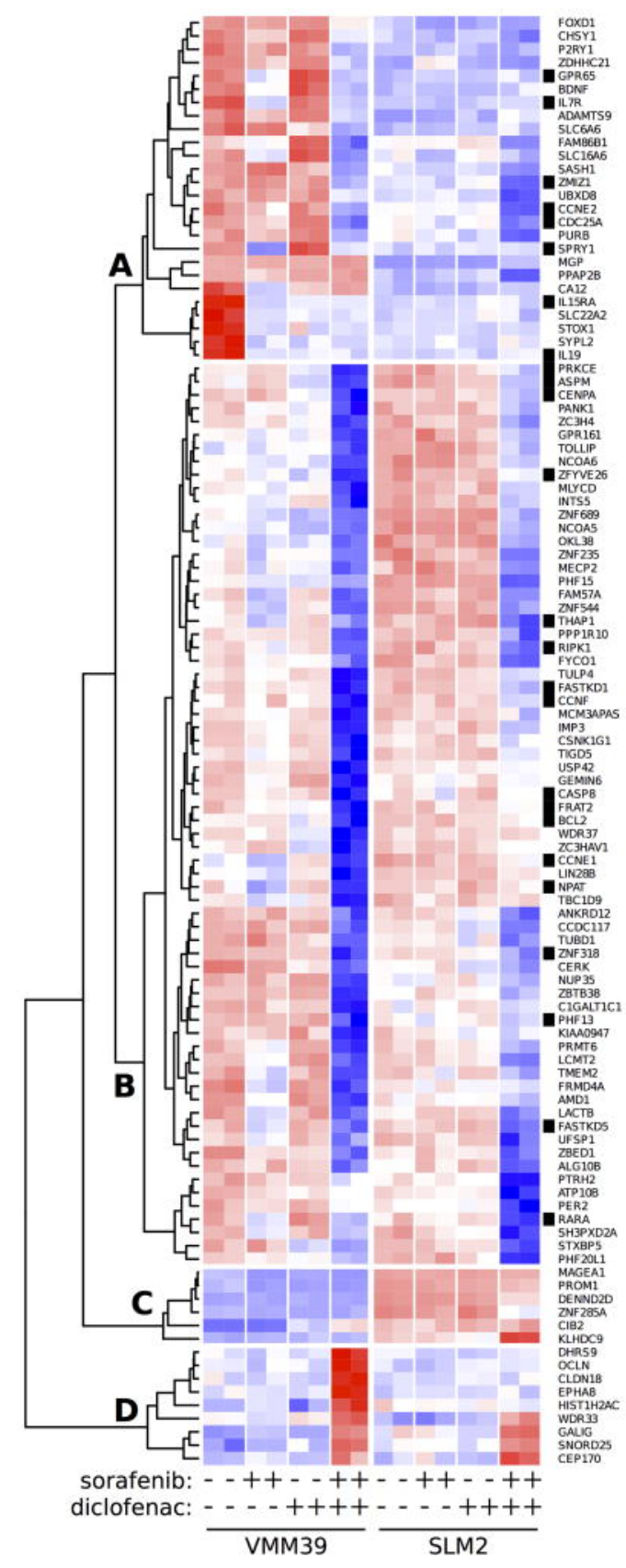

Figure 4.1 The effect of drug combinations on genome-wide gene expression.

Total RNA was isolated from cells (VMM39 and SLM2) 24 hours following inhibitor treatment (dimethyl sulfoxide vehicle, sorafenib and diclofenac, both alone and in combination), conducted in duplicate. mRNA abundance was measured by Illumina HT-12 microarray. Genes exhibiting statistically significant $(F D R<0.1 \%)$ inhibitorinduced changes were clustered hierarchically by average linkage of scaled cosine correlation similarity, delineating 4 major response patterns: A, genes strongly downregulated by combination inhibition in VMM39, but weakly (or not at all) in SLM2. B, genes downregulated by combination inhibition in both VMM39 and SLM2, with varying magnitude. $C$, genes with widely disparate basal expression levels between VMM39 and SLM2 that are dysregulated by combination inhibition. D, genes 
that are upregulated by combination inhibition in either/both VMM39 and SLM2. Black boxes to the left of HUGO gene names denote those genes having annotated associations with survival-related GO terms. Samples were prepared by Devin Roller, in the Weber lab at the University of Virginia

When we examined the broader trends of the gene expression responses to the single and combination treatments of sorafenib and diclofenac, we observed a result dissimilar to what we saw from the combination of PLX4720 and RTK inhibitors. Neither treatment with sorafenib or diclofenac alone appeared to yield a robust adaptive transcriptional response as we had observed with PLX4720. Neither cell line showed excessive cytotoxicity in response to sorafenib or diclofenac treatment alone, indicating there are adaptive responses, which are preventing apoptosis. We had observed that RTK inhibition appears to potentiate the transcriptomic response to BRaf inhibition in the context of treatment with PLX4720; in this case, Cox2 inhibition and multikinase inhibition appear to cause the emergence of new genes not affected by either drug, sorafenib or diclofenac, alone. The mechanism is unknown by which the inhibition of these two targets synergistically induces the apoptosis we observe in our panel of melanomas. The effectiveness of the previous combination of PLX4720 with lapatinib, and the effectiveness of this combination of sorafenib and diclofenac, suggest that synergistic combinations could be arrived upon regardless of the proximity of nodes in biochemical pathways between the targets. This observation suggests a number of further investigations regarding the effectiveness of proximal and distal targets, as well as whether potentiative or emergent responses are more desirable in a clinical setting. Our current experience with these combinations suggests that proximal combinations yield potentiating responses, and distal combinations yield 
emergent responses, but more work is required to prove the hypothesis.

Additionally, it is unclear as to whether these phenomena that we observe are a result of focusing on melanoma or growth driven by the MAPK pathway.

\subsubsection{Synthetic lethal screening approach identifies secondary drugs that, when used in conjunction with ibrutinib, yield highly synergistic cytotoxic responses in MCL cell lines.}

To evaluate our methodology and conclusions from our melanoma studies, we turned towards B-Cell malignancies and sought to identify any parallels. Using a synthetic lethal screening strategy, the Weber lab was able to arrive at a number of targeted therapies, that, when combined with ibrutinib, showed synergistic response in MCL cell lines JVM2 and Z138. Ibrutinib is an inhibitor targeting BTK, a downstream effector of the BCR signaling pathway. In this screen, we used ABT199, a BCL2 inhibitor; bendamustine, an alkylating agent; carfilzomib, a proteasome inhibitor; bortezomib, a proteasome inhibitor; geldanamycin, an HSP90 inhibitor; panobinostat, a non-selective histone deacetylase inhibitor; SC514, an IKK inihibitor; PD325901, a MEK1/2 inhibitor; PD0332991, a CDK inhibitor; enzastaurin, a PKC beta inhibitor; R788, a Syk inhibitor; temsirolimus, an mTOR inhibitor; dasatinib, a BCR/ABL and Src family tyrosine kinase inhibitor; and idelalisib, a PI3K inhibitor (Table 4.1). For each combination across the three by three dose format, the synergy scores, as determined by the Bliss test of independence, were subjected to unsupervised hierarchical clustering (Figure 4.2).

Table 4.1 Secondary drugs used in combination with ibrutinib

\begin{tabular}{|c|c|c|c|}
\hline Drug & Target & Relationship to BCR pathway & Doses used \\
\hline Idelalisib (GS-1101, CAL-101) & PI3K & Proximal & $0.3125,0.625,1.25 \mu \mathrm{M}$ \\
\hline Dasatinib & LYN & Proximal & $6.25,25,100 \mu \mathrm{M}$ \\
\hline
\end{tabular}




\begin{tabular}{|c|c|c|c|}
\hline Enzastaurin & $\mathrm{PKC}$ & Proximal & $2.5,5,10 \mu \mathrm{M}$ \\
\hline SC-514 & IKK & Proximal & $6.25,12.5,25 \mu \mathrm{M}$ \\
\hline R-788 & SYK & Proximal & $0.6,1.35,2.85 \mu \mathrm{M}$ \\
\hline PD-0332991 & $\mathrm{CDK}$ & Distal & $125,250,500 \mathrm{nM}$ \\
\hline PD-325901 & MEK & Distal & $6.25,12.5,25 \mathrm{nM}$ \\
\hline Geldanamycin & Chaperone & Distal & $3.9,7.8,15.6 \mathrm{nM}$ \\
\hline Bortezomib & Proteasome & Distal & $1,1.5,2 \mathrm{nM}$ \\
\hline Carfilzomib & Proteasome & Distal & $1.3,2.6,5.2 \mathrm{nM}$ \\
\hline ABT-199 & BCL-2 & Distal & $0.75,1.25,2.5 \mu \mathrm{M}$ \\
\hline Bendamustine & DNA replication & Distal & $3.125,6.25,12.5 \mu \mathrm{M}$ \\
\hline
\end{tabular}

We observed a striking relationship between the effectiveness of the secondary drugs in combination with ibrutinib (Figure 4.2). The most synergistic secondary drugs were all inhibitors of target proteins that are not directly tied to BCR signaling (Reviewed in Chapter 1). The least synergistic secondary drugs were all targeted inhibitors of members of the BCR signaling pathway, for example, idelalisib. This indicates that for MCL and potentially other B-Cell malignancies, drug combinations should target proteins that are components of different pathways. This runs contrary to results in melanoma that repeated insults to the MAPK pathway, the primary growth pathway in our melanoma cell lines and the analogous pathway to BCR signaling, are an effective strategy for overcoming resistance. We next wanted to determine the pathways that are being affected by the treatment to induce apoptosis. 

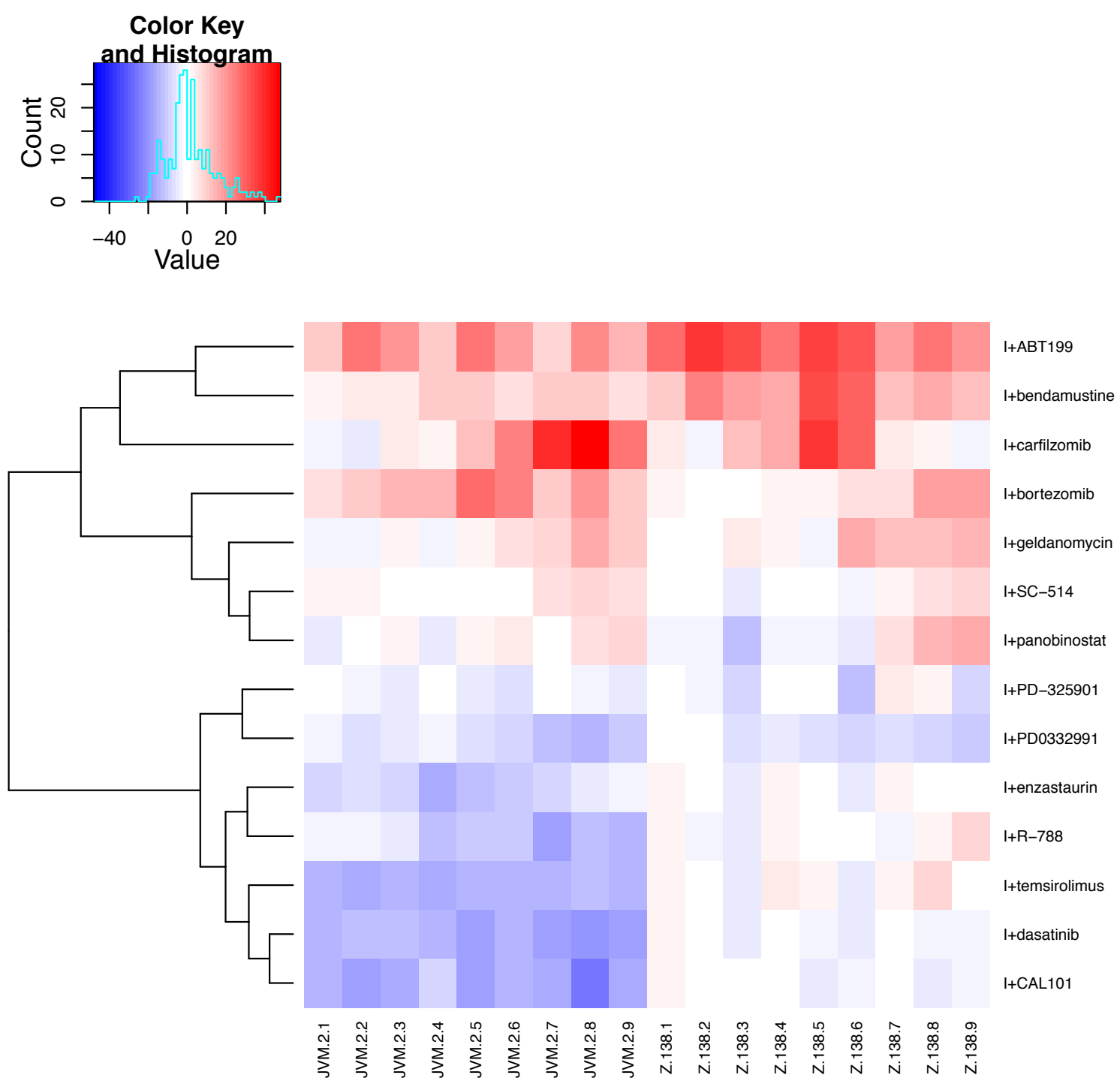

Figure 4.2 Screening with targeted secondary agents identifies drug combinations that synergize with ibrutinib.

Fourteen secondary drugs were combined with ibrutinib in two MCL cell lines (Z138 and JVM2). Cell lines were exposed to 6, 12 and $21 \mu$ M ibrutinib and three doses of secondary agents in a $3 \times 3$ format for $72 \mathrm{~h}$. Percent cytotoxicity was measured with an alamarBlue assay, and percent synergy assessed by the Bliss independence method. Unsupervised hierarchical clustering of the percent synergy values for all drug combinations at all concentration combinations in the $3 \times 3$ dosing matrix in Z138 and JVM2 cells is shown. Samples were prepared by Mark Axelrod and Vicki Gordon, in the Weber lab at the University of Virginia. 


\subsubsection{Transcriptomic responses to combination treatments reveal potential}

mechanisms of synergistic cytotoxic effects.

To ascertain the transcriptional responses of the cell lines to these

combination treatments, we performed a gene expression array 6 hours post

treatment with ibrutinib at two doses in combination with single doses of

carfilzomib, idelasib, and ABT-199. At a FDR threshold of 1\%, we identified a

number of genes differentially expressed in all drug treatments in both cell lines, when compared to control (Figure 4.3). Across the two MCL lines we studied, only JVM2 under high dose ibrutinib treatment showed a robust adaptive gene expression response. Much like sorafenib treatment in our non-mutant BRAF melanoma lines, and PLX4720 treatment in our BRAFV600E lines, each line displays mostly unique responses to treatment. Additionally, the response to the secondary drugs is extremely limited, again, similar to the result of laptinib, masitinib, and diclofenac treatment in our melanoma panels. Lastly, we do observe blocks of genes responding in kind after treatment with the combination, across each of the different secondary drugs. Each of these secondary drugs target different pathways, which would seem to indicate that we are observing a readout of synergistic cytotoxicity, rather than observing the mechanisms that result in synergistic cytotoxicity. 

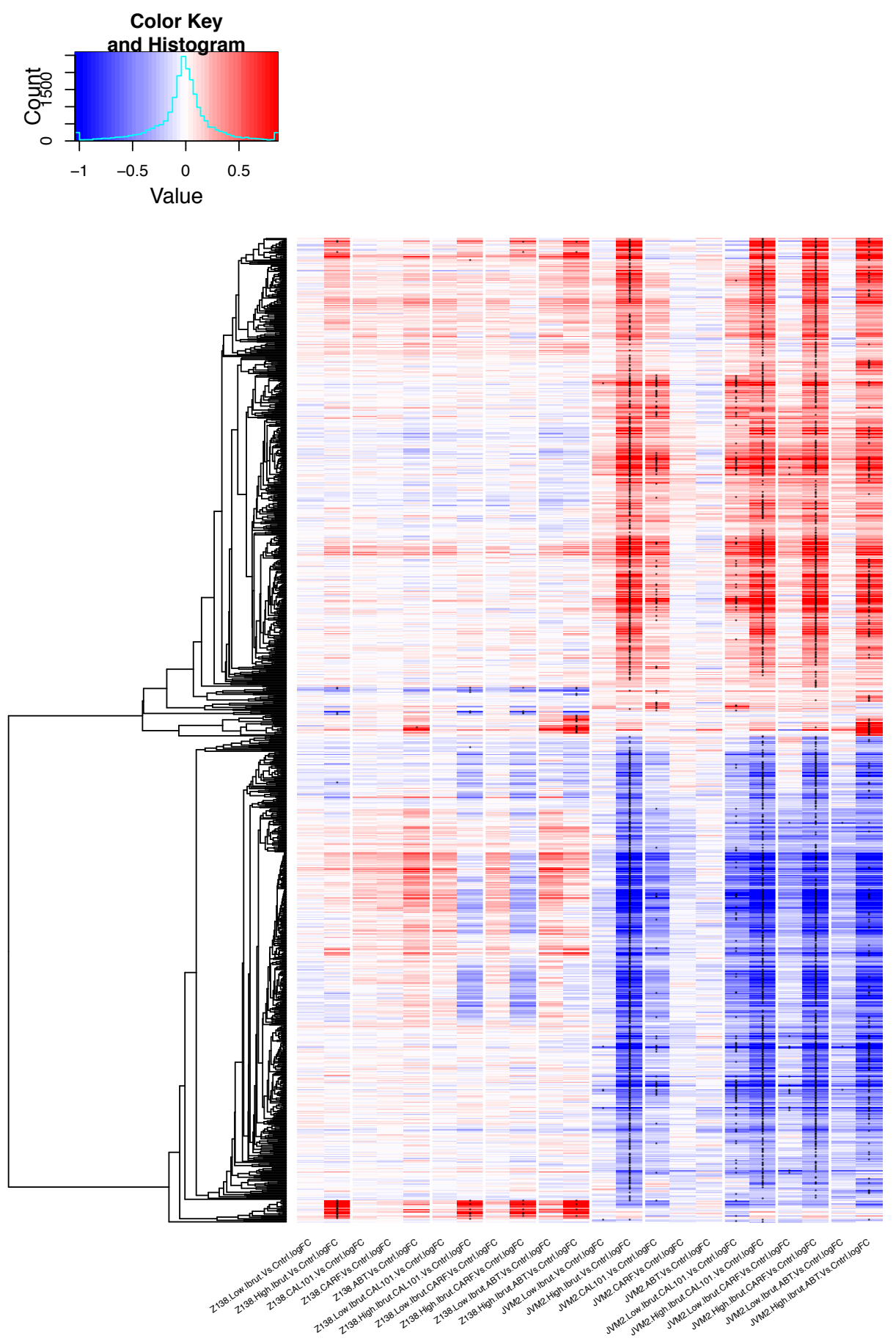

Figure 4.3 Synergistic combinations in MCL lines exhibit strong transcriptional responses of an emergent nature

Moderated t-test was used to identify differentially expressed genes in response to treatment with ibrutinib alone at a low (low.Ibrut) and high (high.Ibrut) dose, CAL101 (CAL101) alone, Carfilzomib alone (CARF), ABT199 (ABT) alone, or combinations of 
ibrutinib with each of the secondary drugs in two MCL cell lines (JVM2, Z138). Samples were exposed to treatments for 8 hours. Differentially expressed genes with a FDR or $1 \%$ or better are denoted with “*”. Samples were prepared by Vicki Gordon, in the Weber lab at the University of Virginia.

We focused our analyses of the transcriptomic response in JVM2 to the combination of ibrutinib with idelasib, chosen because, although it was one of the least effective ibrutinib combinations from a cytotoxicity perspective (Figure 4.2), it was the combination treatment that yielded the highest number of differentially expressed genes. We took the list of differentially expressed genes and performed a pathway enrichment analysis using MSigDB (Table 4.2). Among the top pathways, we see MAPK pathway signaling and p53 signaling. Since idelasib is a PI3K inhibitor, upregulation of MAPK signaling is not unexpected. It is well documented that inhibiting PI3K signaling will often upregulate MAPK signaling, and the reverse has also been observed, even in our melanoma cell lines when treated with PLX4720, inducing the adaptive signaling response (Chandarlapaty et al., 2011; Kugel et al., 2014). The p53 responses are also expected; even though the combination of ibrutinib and idelasib is not synergistic, it is still cytotoxic, which could require the induction of p53 signaling to induce apoptosis. Transcription factor enrichment analysis reveals forkhead box family members, ETS family members and their cofactors, and the NFkB and STAT transcription factors. Given the similar enrichments of ETS family and forkhead box family members that we observed in melanoma, it is not unexpected to observe that enrichment here, considering the previously-mentioned enrichment of MAPK signaling present in the pathway enrichment. The combination of p53 induction coupled with the potentially robust MAPK signaling induction could explain the poor synergistic response of the 
combination of ibrutinib with idelasib. These results seem to indicate the induction

of both apoptosis and growth at the same time.

Table 4.2 MSigDB pathway enrichment results for differentially expressed genes in response to treatment with Ibrutinib and secondary drugs in JVM2

\begin{tabular}{|c|c|c|c|}
\hline Gene Set Name & Description & FDR q-value & Secondary Drug \\
\hline REACTOME_IMMUNE_SYSTEM & Genes involved in Immune System & $3.51 \mathrm{E}-10$ & Idelasib \\
\hline $\begin{array}{l}\text { REACTOME_CYTOKINE_SIGNALI } \\
\text { NG_IN_IMMUNE_SYSTEM }\end{array}$ & $\begin{array}{l}\text { Genes involved in Cytokine Signaling in } \\
\text { Immune system }\end{array}$ & $4.38 \mathrm{E}-09$ & Idelasib \\
\hline $\begin{array}{l}\text { REACTOME_INTERFERON_SIGN } \\
\text { ALING }\end{array}$ & Genes involved in Interferon Signaling & 4.38E-09 & Idelasib \\
\hline $\begin{array}{c}\text { KEGG_P53_SIGNALING_PATHW } \\
\text { AY }\end{array}$ & p53 signaling pathway & $4.38 \mathrm{E}-09$ & Idelasib \\
\hline $\begin{array}{l}\text { REACTOME_INTERFERON_GAM } \\
\text { MA_SIGNALING }\end{array}$ & $\begin{array}{c}\text { Genes involved in Interferon gamma } \\
\text { signaling }\end{array}$ & $3.13 \mathrm{E}-07$ & Idelasib \\
\hline $\begin{array}{l}\text { REACTOME_INTERFERON_ALPH } \\
\text { A_BETA_SIGNALING }\end{array}$ & $\begin{array}{l}\text { Genes involved in Interferon } \\
\text { alpha/beta signaling }\end{array}$ & 4.57E-06 & Idelasib \\
\hline $\begin{array}{c}\text { KEGG_MAPK_SIGNALING_PATH } \\
\text { WAY }\end{array}$ & MAPK signaling pathway & $1.04 \mathrm{E}-05$ & Idelasib \\
\hline $\begin{array}{l}\text { KEGG_CYTOKINE_CYTOKINE_RE } \\
\text { CEPTOR_INTERACTION }\end{array}$ & Cytokine-cytokine receptor interaction & $4.80 \mathrm{E}-05$ & Idelasib \\
\hline PID_HIV_NEF_PATHWAY & $\begin{array}{l}\text { HIV-1 Nef: Negative effector of Fas and } \\
\text { TNF-alpha }\end{array}$ & 7.19E-05 & Idelasib \\
\hline $\begin{array}{l}\text { PID_P53_DOWNSTREAM_PATH } \\
\text { WAY }\end{array}$ & Direct p53 effectors & 7.67E-05 & Idelasib \\
\hline REACTOME_IMMUNE_SYSTEM & Genes involved in Immune System & $1.61 \mathrm{E}-07$ & Carfilzomib \\
\hline $\begin{array}{c}\text { KEGG_P53_SIGNALING_PATHW } \\
\text { AY }\end{array}$ & p53 signaling pathway & $1.97 \mathrm{E}-07$ & Carfilzomib \\
\hline $\begin{array}{l}\text { KEGG_JAK_STAT_SIGNALING_P } \\
\text { ATHWAY }\end{array}$ & Jak-STAT signaling pathway & 3.77E-06 & Carfilzomib \\
\hline $\begin{array}{l}\text { KEGG_CYTOKINE_CYTOKINE_RE } \\
\text { CEPTOR_INTERACTION }\end{array}$ & Cytokine-cytokine receptor interaction & $4.98 \mathrm{E}-06$ & Carfilzomib \\
\hline PID_MYC_ACTIV_PATHWAY & $\begin{array}{l}\text { Validated targets of C-MYC } \\
\text { transcriptional activation }\end{array}$ & $4.98 \mathrm{E}-06$ & Carfilzomib \\
\hline $\begin{array}{l}\text { REACTOME_CYTOKINE_SIGNALI } \\
\text { NG_IN_IMMUNE_SYSTEM }\end{array}$ & $\begin{array}{l}\text { Genes involved in Cytokine Signaling in } \\
\text { Immune system }\end{array}$ & 2.17E-05 & Carfilzomib \\
\hline BIOCARTA_HIVNEF_PATHWAY & $\begin{array}{c}\text { HIV-I Nef: negative effector of Fas and } \\
\text { TNF }\end{array}$ & $2.70 \mathrm{E}-05$ & Carfilzomib \\
\hline $\begin{array}{c}\text { KEGG_TYPE_I_DIABETES_MELLI } \\
\text { TUS }\end{array}$ & Type I diabetes mellitus & $2.97 \mathrm{E}-05$ & Carfilzomib \\
\hline $\begin{array}{l}\text { REACTOME_TRANSMEMBRANE } \\
\text { _TRANSPORT_OF_SMALL_MOL } \\
\text { ECULES }\end{array}$ & $\begin{array}{l}\text { Genes involved in Transmembrane } \\
\text { transport of small molecules }\end{array}$ & $2.97 \mathrm{E}-05$ & Carfilzomib \\
\hline $\begin{array}{l}\text { REACTOME_UNFOLDED_PROTEI } \\
\text { N_RESPONSE }\end{array}$ & $\begin{array}{l}\text { Genes involved in Unfolded Protein } \\
\text { Response }\end{array}$ & $3.14 \mathrm{E}-05$ & Carfilzomib \\
\hline $\begin{array}{l}\text { REACTOME_CYTOKINE_SIGNALI } \\
\text { NG_IN_IMMUNE_SYSTEM }\end{array}$ & $\begin{array}{l}\text { Genes involved in Cytokine Signaling in } \\
\text { Immune system }\end{array}$ & $2.36 \mathrm{E}-07$ & ABT-199 \\
\hline $\begin{array}{l}\text { REACTOME_INTERFERON_SIGN } \\
\text { ALING }\end{array}$ & Genes involved in Interferon Signaling & 3.57E-07 & ABT-199 \\
\hline REACTOME_IMMUNE_SYSTEM & Genes involved in Immune System & $3.57 \mathrm{E}-07$ & ABT-199 \\
\hline $\begin{array}{c}\text { KEGG_P53_SIGNALING_PATHW } \\
\text { AY }\end{array}$ & p53 signaling pathway & $9.42 \mathrm{E}-07$ & ABT-199 \\
\hline $\begin{array}{l}\text { KEGG_CYTOKINE_CYTOKINE_RE } \\
\text { CEPTOR_INTERACTION }\end{array}$ & Cytokine-cytokine receptor interaction & $4.78 \mathrm{E}-06$ & ABT-199 \\
\hline $\begin{array}{c}\text { KEGG_MAPK_SIGNALING_PATH } \\
\text { WAY }\end{array}$ & MAPK signaling pathway & $3.43 \mathrm{E}-05$ & ABT-199 \\
\hline $\begin{array}{l}\text { KEGG_INTESTINAL_IMMUNE_N } \\
\text { ETWORK_FOR_IGA_PRODUCTI }\end{array}$ & $\begin{array}{l}\text { Intestinal immune network for IgA } \\
\text { production }\end{array}$ & $3.43 \mathrm{E}-05$ & ABT-199 \\
\hline
\end{tabular}




\begin{tabular}{|c|c|c|c|}
\hline ON & A & & ABT-199 \\
\hline $\begin{array}{c}\text { REACTOME_INTERFERON_ALPH } \\
\text { A_BETA_SIGNALING }\end{array}$ & $\begin{array}{c}\text { Genes involved in Interferon } \\
\text { alpha/beta signaling }\end{array}$ & $1.71 \mathrm{E}-04$ & ABT-199 \\
\hline PID_CD8_TCR_PATHWAY & $\begin{array}{c}\text { TCR signaling in na\&\#xef;ve CD8+ T } \\
\text { cells }\end{array}$ & $1.18 \mathrm{E}-03$ & ABT-199 \\
\hline $\begin{array}{c}\text { KEGG_SYSTEMIC_LUPUS_ERYTH } \\
\text { EMATOSUS }\end{array}$ & Systemic lupus erythematosus & $1.18 \mathrm{E}-03$ & \\
\hline
\end{tabular}

\subsubsection{The combination of carfilzomib with ibrutinib appears to induce an extremely strong and robust apoptotic response via p53 signaling. We next sought to examine the transcriptional responses induced by the}

combination of ibrutinib and carfilzomib in the JVM2 cell line at the pathway level of response. The pathway enrichment results were extremely striking: we observed multiple pathways associated with p53 and its ability to effect apoptosis. Even more intriguing was that the combination of carfilzomib with ibrutinib involved the response of many genes that were emergent in their response to the combination (Figure 4.3). When we focused exclusively on the genes that were emergent in the combination, we still observe the p53 response (driven by downregulation of cyclin D2 and cyclin E1, which had $\log 2$ fold changes of -0.60 and -0.27 respectively; and upregulation of cyclin G2, with a log2 fold change of 0.47 ). We also observe a significant induction ( $\log 2$ fold change of 0.34 ) of GADD45A, a growth arrest and DNA damage inducible protein. Contrary to the response we observe in the combination of idelasib with ibrutinib, we see no induction of alternative growth pathways. This would agree with our assessment of the more synergistic nature of the combination ibrutinib with carfilzomib. Comparing the two combinations demonstrates the significance of using combinations that target different pathways. With idelasib, the BCR growth pathway is targeted at two points, BTK and PI3K. This heavy inhibition of the BCR pathway appears to cause apoptosis, but also induces 
the growth response via MAPK pathway signaling, an adaptive response similar to PLX4720 treatment in melanoma. Utilizing carfilzomib, an inhibitor of the proteasome, in conjunction with BTK inhibition appears to yield a much stronger apoptotic response, avoiding the induction of alternative growth pathways.

\subsubsection{The combination of ABT-199 and ibrutinib induces a transcriptional response similar to the combination of idelasib and ibrutinib. \\ When we sought to categorize the pathways affected by the combination of} high dose ibrutinib with ABT-199 in the emergent genes, we again discovered that this combination centers on apoptosis via p53 induction. Even though this combination is the most robust as scored by Bliss, the response in JVM2 is very similar to its response to the combination of idelasib and ibrutinib in terms of the pathways induced by the treatments. Again we see an enrichment of p53 associated pathways and PI3K pathways. Additionally, when we perform a pathway enrichment analysis only on emergent genes, we lose the enrichment in p53 pathways, but maintain the PI3K pathway enrichment. We also appear to maintain similar transcriptional programs in both combinations, with enrichments in forkhead box family members and in transcription factors with MAPK pathway consensus sequences (Diella et al., 2008). These results were very unexpected, as the combination with ABT-199 was one of the most synergistic combinations, far more effective than the combination with idelasib. Across all three combinations, we observe enrichment in p53-associated pathways. This is unusual as all three secondary drugs target different pathways. We would expect different pathways enriched for different combinations due to the different targets of the secondary drugs. In our profiling, we appear to only be observing the end result of cytotoxicity 
in all three combinations. This would seem to indicate that our gene expression profiling is not sufficient to capture the adaptive response, if any, in our model of BCell malignancies. The combination of ABT-199 and ibrutinib, being the most synergistic combination and showing similar responses to treatment of the other combinations became our primary focus. We wanted to determine the protein signaling changes being induced by treatment to potentially further elucidate the mechanisms of cytotoxicity and synergy.

\subsubsection{The apoptotic response to the combination of ABT-199 and ibrutinib is very swift, and is apparent when observed at the protein level. To ascertain responses to these treatments at the protein level, we used a} reverse phase protein array, examining the responses of approximately 100 proteins at 1, 3, 6, and 24 hours for all single and combination treatments (Figure 4.4). In the JVM line, we were able to see evidence of apoptosis as early as $3 \mathrm{hrs}$ in response to ABT-199 treatment alone. At 3 hours, signals of ABT-199's apoptosistriggering effects were most apparent via increases in cleaved caspase family members. When observing the combination treatment at 3 hours, induction of apoptosis appears even stronger. Levels of cleaved caspase family members are even higher than in ABT-199 treatment alone. This increase persists at every time point from 3 hours to 24 hours post treatment. Lastly, combination treatment significantly lowers the phosphorylation levels of multiple receptor tyrosine kinases responsible for triggering growth and survival, as well as levels of many members of the downstream effectors of these growth pathways. 

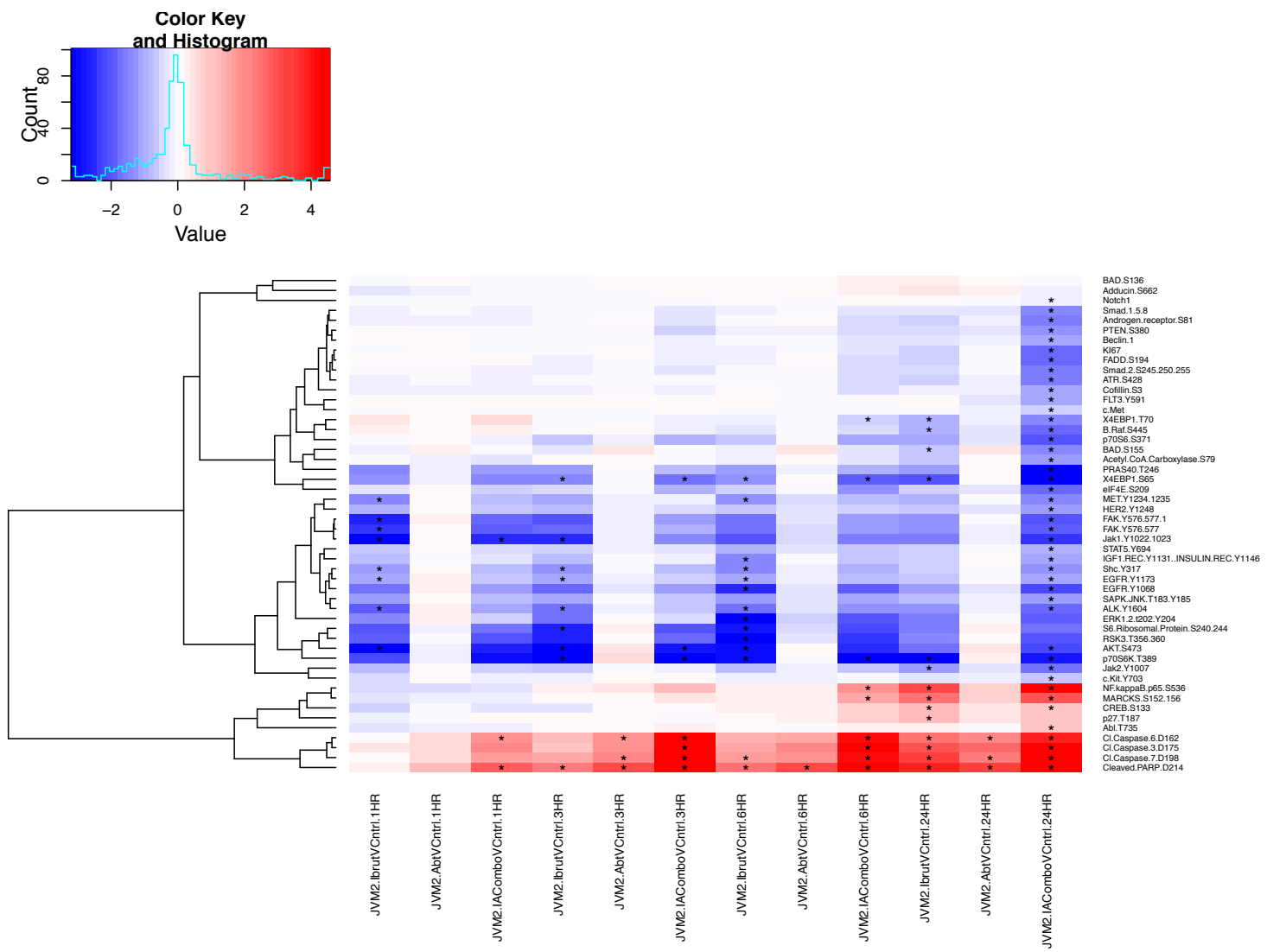

Figure 4.4 ABT199 treatment appears to induce apoptosis very swiftly

Moderated t-test was performed on normalized, log2 transformed RPPA levels. Log2 fold changes were plotted for JVM2 cells treated with ibrutinib alone (Ibrut), ABT199 alone (Abt), or the combination (IACombo). Responses were assayed at 1, 3, 6, and 24 hours. Fold changes with a FDR of 1\% or better are denoted with "*”. Samples were prepared by Vicki Gordon, in the Weber lab at the University of Virginia.

These results indicate that observing the response to the combination in the transcriptome at 6 hours might be misleading. Given the strong apoptotic results at 3 hours, it is very apparent that transcriptional responses at 6 hours could be heavily contaminated with dead cells and apoptotic byproducts. Additionally, it implies that the adaptive response in the transcriptome would be occurring much earlier than previously assayed. 


\subsubsection{Transcriptomic response to ibrutinib and ABT-199 combination}

reveals little insight into the mechanism of synergy.

Given the striking result that apoptosis is triggered as early as 3 hours post

treatment, we wanted to investigate the effects on the transcriptome at this time.

We performed another gene expression array, again on JVM2, and on two other MCL cell lines, Mino and Jeko (Figure 4.5). As with Z138, JVM2 shows a marked increase in the number of genes that respond to treatment compared to the other cell lines' responses. We examined the transcription factors that contain potential binding sites in the genes in JVM2 that are significantly affected by ABT-199 treatment, and observe a number of factors associated with MAPK signaling, including ELK1 and ETS2, both of which are ETS family members; SP1, an ETS family cofactor; and NFAT, a target of the MAPK pathway. As discussed in the previous chapter, ETS transcription factors are the primary family affected by MAPK signaling. We next wanted to ascertain which pathways are most affected by ABT-199 treatment. As previously observed, apoptosis, via the p53 signaling pathway, is among the most significant effects of ABT-199 treatment (FDR = 1.6 e -3). But we also observe ABT199 effects on a number of receptor tyrosine kinase pathways, including ErbB signaling, PDGF signaling, and glucocorticoid receptor signaling. We also observe effects on a number of cell cycle associated pathways and cell stress pathways, such as oxidative phosphorylation and protein metabolism. Taken together, these pathways, specifically p53 and oxidative phosphorylation, would seem to indicate strong induction of an apoptotic response to ABT-199 treatment. 

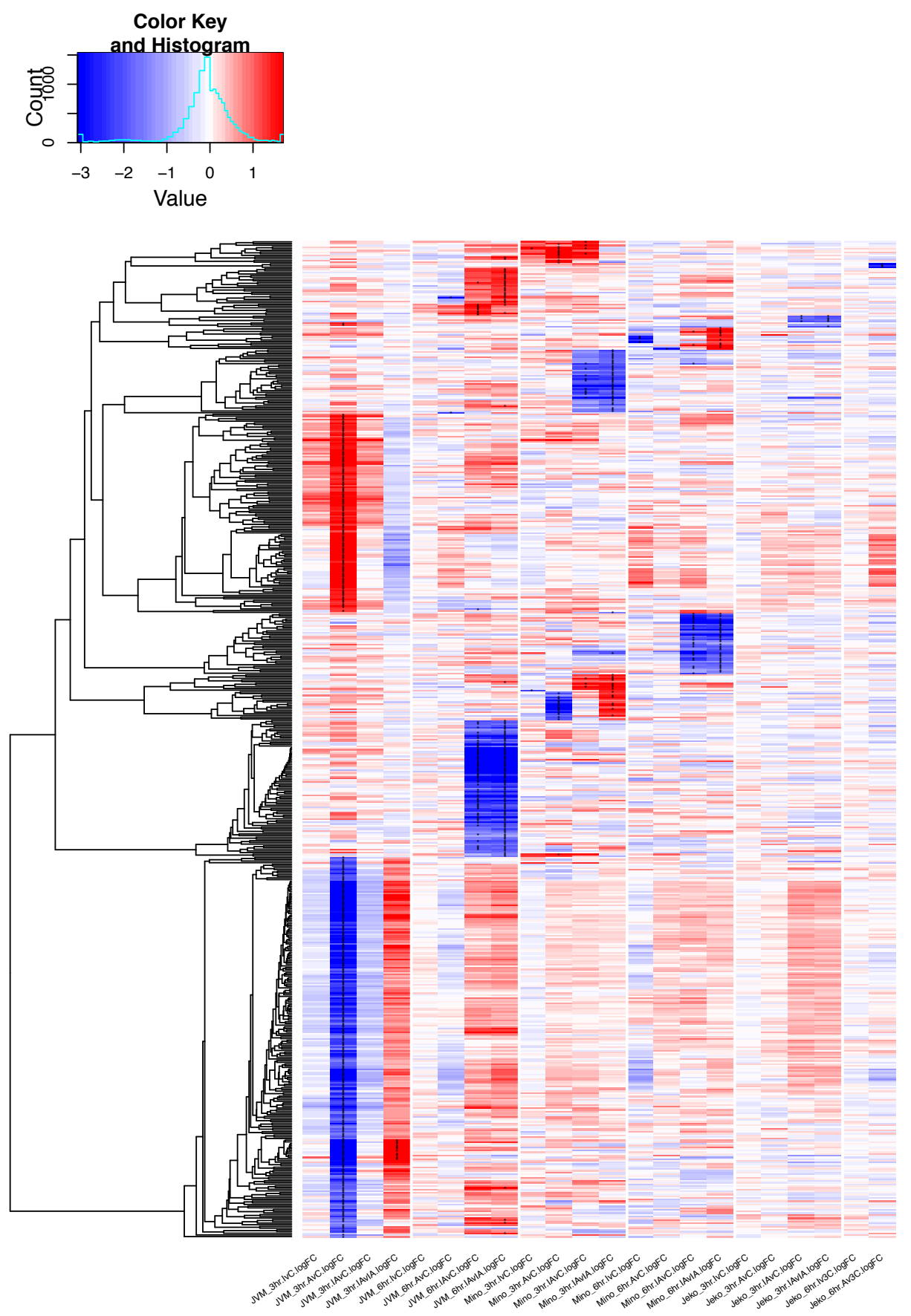

Figure 4.5 Transcriptomic responses to Ibrutinib and ABT at time of apoptosis Moderated $t$-test was used to identify differentially expressed genes in response to treatment with ibrutinib (IvC), ABT199 (AvC) alone, the combination of ibrutinib with ABT199 (IAvC), or a measure of synergy (IAvIA) defined in methods in JVM2 (JVM), Mino, and Jeko MCL cell lines. Differentially expressed genes with a FDR or 1\% or 
better are denoted with “*”. Samples were prepared by Vicki Gordon, in the Weber lab at the University of Virginia.

More strikingly, we see little to no significant responses to other single treatments at 3 hours in any of the lines. We do observe a much stronger combination response at 6 hours in JVM2 compared to the other lines, and the genes affected mostly appear to be responding opposite to the ABT-199 response at 3 hours. It would appear, as we saw in our melanoma studies, that JVM2 is attempting to overcome single drug treatment utilizing an adaptive response. We see a limited induction of growth pathways in the form MAPK signaling and the apparent transcriptional response of MAPK pathway activation. Given the strong in vitro response of MCL cell lines to the combination of ABT-199 and ibrutinib, we sought to ascertain the effectiveness of this combination in patient samples.

\subsubsection{Patient samples show significant responses to the combination of} ABT199 and ibrutinib in vitro.

We began our studies by assaying for cleaved PARP, an indicator of apoptotic response, in a number of CLL and MCL patient samples as well as in normal patient tissue: for ibrutinib treatment alone, ABT-199 treatment alone, and the combination (Figure 4.6). As we saw in cell lines, we observe minimal apoptosis in response to ibrutinib treatment alone, a marked increase in cleaved PARP with the treatment of the samples with ABT-199, and, with treatment by the combination, a striking increase in cleaved PARP, beyond the increase observed with ABT-199 alone. This observation strongly indicates that the combination of ABT-199 and ibrutinib would be useful for patients stricken with either CLL or MCL. We rankordered the patient samples from most to least responsive to the combination, and 
observed that even patients who showed limited response to either ibrutinib or ABT-199 alone were responsive to the combination. However, patient samples that were most resistant to ibrutinib also appeared to be the most resistant to the combination. 

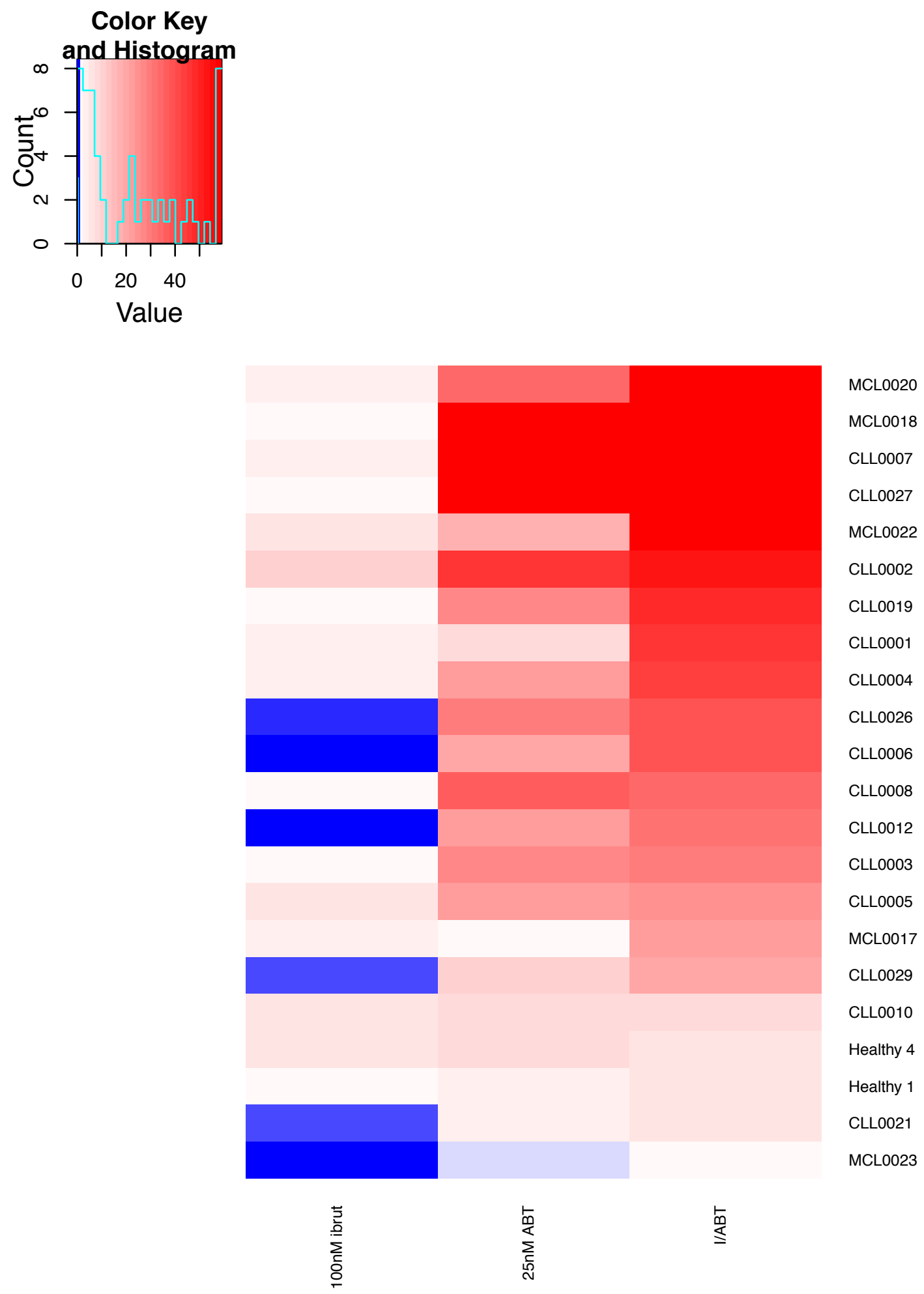

Figure 4.6 Ibrutinib combined with ABT-199 shows strong response is ex vivo models of patient samples

Cleaved PARP was assayed as a means of determining apoptotic effects of ibrutinib alone, ABT199 alone, or the combination in patient samples. Levels of cleaved PARP 
have been normalized to levels of cleaved PARP observed in the patient samples under no treatment condition. Samples were prepared by Vicki Gordon, in the Weber lab at the University of Virginia.

Having determined that patient samples respond to the combination, we sought to examine the responses in patient samples and assay the similarity in these responses to the responses observed in cell lines. Three samples were taken through further analyses to determine the similarity. These three were MCL0020, the most responsive patient sample to the combination; CLL0021, one of the patient samples most resistant to ibrutinib treatment, resistant to ABT-199 treatment, and among the least responsive to the combination; and CLL0026, a patient sample that was extremely resistant to ibrutinib treatment alone but somewhat responsive to ABT-199 treatment and to the combination treatment. We felt that these three samples were a fair representation of the variation in responses to treatment that we observed for all patient samples.

\subsubsection{Patient sample transcriptomic response to the combination yields similar results to cell line models.}

Given the responses observed in the gene expression array and protein array

for the cell lines, we decided that gene expression would be the best avenue for studying the patient sample responses. The protein array analysis did demonstrate the rapid induction of apoptosis, but it does not provide sufficient analytes to undergo extensive pathway analysis. We began with an analysis of the patient samples' responses to the single and combination treatments (Figure 4.7). In MCL0020, we see a very robust response to ABT-199 and combination treatment, and a weak response to ibrutinib treatment alone, which mirrors its cleaved PARP assay results. Additionally, we observe a number of emergent responses to the 
combination treatment. When looking at the genes that are differentially expressed after combination treatment, we observe high numbers of micro and small nucleolar RNA molecules, a response we do not often observe. As before, we wanted to determine the specific pathways and transcription factors being induced by treatment (Table 4.3). We observe a significant enrichment only in the p38 gamma and p38 delta signaling pathway. This enrichment is due to MAPK12, MAPK13 and STMN1 being differentially expressed in response to combination treatment. This pathway is associated with the stress response, which could be a potential indication of apoptosis. We also observe pathways associated with Pol I, packaging of telomere ends, and mitotic recombination. Notably, dysregulation of telomers can lead to apoptosis (Ci et al., 2015). Performing the transcription factor binding site enrichment analysis, we identify multiple transcription factors that contain MAPK consensus sequences, as we observed before, including NFAT and MAZ. We also observe enrichment for NFKB and associated cofactors; STAT family members STAT5A and STAT6; and a number of forkhead box transcription factor family members, including FOXA1, FOXA2, FOXD3, and FOXO4. 

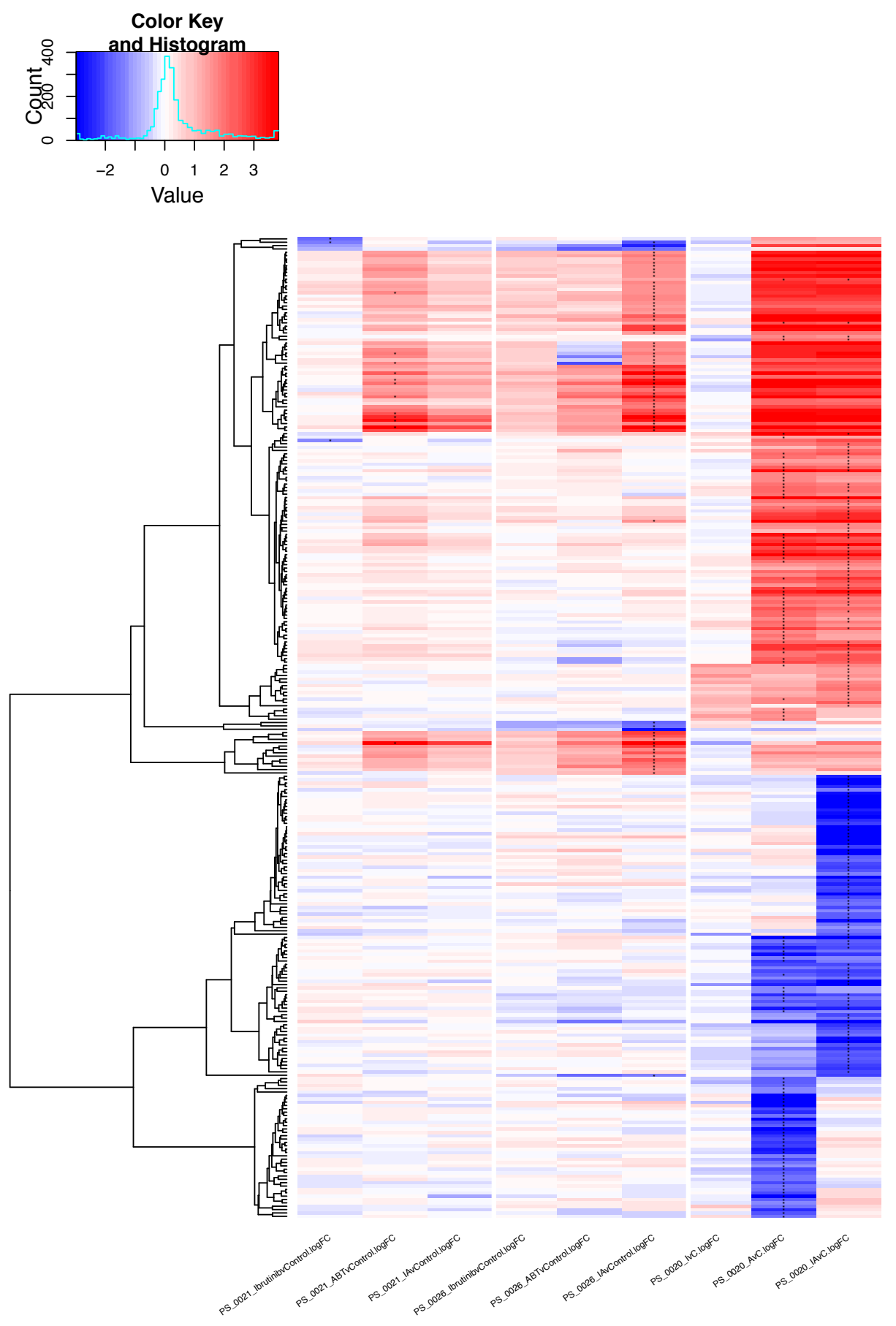

Figure 4.7 Ex vivo response to combination in patient samples is extremely pronounced

Moderated t-test was used to identify differentially expressed genes in response to treatment with ibrutinib (IbrutinibvControl), ABT199 (ABTvControl) alone, or the combination of ibrutinib with ABT199 (IAvControl) in patient samples. Differentially 
expressed genes with a FDR or 1\% or better are denoted with “*”. Samples were prepared by Vicki Gordon, in the Weber lab at the University of Virginia.

\section{Table 4.3 MSigDB enrichment results for ex vivo study on patient samples exposed to ibrutinib and ABT-199}

\begin{tabular}{|c|c|c|c|}
\hline Gene Set Name & Description & FDR q-value & $\begin{array}{l}\text { Patient } \\
\text { Number }\end{array}$ \\
\hline REACTOME AMYLOIDS & Genes involved in Amyloids & $5.20 \mathrm{E}-21$ & CLL 0026 \\
\hline $\begin{array}{l}\text { REACTOME_RNA_POL_I_PROMO } \\
\text { TER_OPENING }\end{array}$ & Genes involved in RNA Polymerase I Promoter Opening & $1.04 \mathrm{E}-20$ & CLL_0026 \\
\hline $\begin{array}{l}\text { REACTOME_MEIOTIC_RECOMBIN } \\
\text { ATION }\end{array}$ & Genes involved in Meiotic Recombination & 4.77E-19 & CLL_0026 \\
\hline $\begin{array}{l}\text { REACTOME_RNA_POL_I_TRANSC } \\
\text { RIPTION }\end{array}$ & Genes involved in RNA Polymerase I Transcription & $5.54 \mathrm{E}-19$ & CLL_0026 \\
\hline $\begin{array}{l}\text { KEGG_SYSTEMIC_LUPUS_ERYTHE } \\
\text { MATOSUS }\end{array}$ & Systemic lupus erythematosus & $1.34 \mathrm{E}-18$ & CLL_0026 \\
\hline $\begin{array}{l}\text { REACTOME_PACKAGING_OF_TEL } \\
\text { OMERE_ENDS }\end{array}$ & Genes involved in Packaging Of Telomere Ends & $8.94 \mathrm{E}-18$ & CLL_0026 \\
\hline REACTOME_MEIOSIS & Genes involved in Meiosis & $8.94 \mathrm{E}-18$ & CLL_0026 \\
\hline $\begin{array}{l}\text { REACTOME_RNA_POL_I_RNA_P } \\
\text { OL_III_AND_MITOCHONDRIAL_T } \\
\text { RANSCRIPTION }\end{array}$ & $\begin{array}{c}\text { Genes involved in RNA Polymerase I, RNA Polymerase III, and } \\
\text { Mitochondrial Transcription }\end{array}$ & $1.47 \mathrm{E}-17$ & CLL_0026 \\
\hline $\begin{array}{l}\text { REACTOME_DEPOSITION_OF_NE } \\
\text { W_CENPA_CONTAINING_NUCLE } \\
\text { OSOMES_AT_THE_CENTROMERE }\end{array}$ & $\begin{array}{l}\text { Genes involved in Deposition of New CENPA-containing } \\
\text { Nucleosomes at the Centromere }\end{array}$ & $1.38 \mathrm{E}-16$ & CLL_0026 \\
\hline REACTOME_MEIOTIC_SYNAPSIS & Genes involved in Meiotic Synapsis & $5.06 \mathrm{E}-16$ & CLL_0026 \\
\hline $\begin{array}{c}\text { KRCTCNNNNMANAGC_UNKNOW } \\
\text { N }\end{array}$ & $\begin{array}{c}\text { Genes with promoter regions }[-2 \mathrm{~kb}, 2 \mathrm{~kb}] \text { around transcription } \\
\text { start site containing motif KRCTCNNNNMANAGC. Motif does } \\
\text { not match any known transcription factor }\end{array}$ & $1.73 \mathrm{E}-11$ & CLL_0026 \\
\hline TTTNNANAGCYR_UNKNOWN & $\begin{array}{c}\text { Genes with promoter regions [-2kb,2kb] around transcription } \\
\text { start site containing motif TTTNNANAGCYR. Motif does not } \\
\text { match any known transcription factor }\end{array}$ & $2.76 \mathrm{E}-09$ & CLL_0026 \\
\hline GTGACGY_V\$E4F1_Q6 & $\begin{array}{l}\text { Genes with promoter regions [-2kb,2kb] around transcription } \\
\text { start site containing the motif GTGACGY which matches } \\
\text { annotation for E4F1: E4F transcription factor } 1\end{array}$ & $3.79 \mathrm{E}-05$ & CLL_0026 \\
\hline V\$SRF_01 & $\begin{array}{l}\text { Genes with promoter regions }[-2 \mathrm{~kb}, 2 \mathrm{~kb}] \text { around transcription } \\
\text { start site containing the motif ATGCCCATATATGGWNNT which } \\
\text { matches annotation for SRF: serum response factor (c-fos serum } \\
\text { response element-binding transcription factor) }\end{array}$ & $8.88 \mathrm{E}-05$ & CLL_0026 \\
\hline TATAAA_V\$STATA_01 & $\begin{array}{c}\text { Genes with promoter regions }[-2 \mathrm{~kb}, 2 \mathrm{~kb}] \text { around transcription } \\
\text { start site containing the motif TATAAA which matches } \\
\text { annotation for TAF }<\text { br }>\text { TATA }\end{array}$ & 7.91E-04 & CLL_0026 \\
\hline V\$SRF_Q5_01 & $\begin{array}{l}\text { Genes with promoter regions [-2kb,2kb] around transcription } \\
\text { start site containing the motif CCAWATAWGGMNMNG which } \\
\text { matches annotation for SRF: serum response factor (c-fos serum } \\
\text { response element-binding transcription factor) }\end{array}$ & $1.09 \mathrm{E}-03$ & CLL_0026 \\
\hline CGTSACG_V\$PAX3_B & $\begin{array}{c}\text { Genes with promoter regions [-2kb,2kb] around transcription } \\
\text { start site containing the motif CGTSACG which matches } \\
\text { annotation for PAX3: paired box gene } 3 \text { (Waardenburg } \\
\text { syndrome } 1 \text { ) }\end{array}$ & $3.85 \mathrm{E}-03$ & CLL_0026 \\
\hline TGANTCA_V\$AP1_C & $\begin{array}{c}\text { Genes with promoter regions [-2kb,2kb] around transcription } \\
\text { start site containing the motif TGANTCA which matches } \\
\text { annotation for JUN: jun oncogene }\end{array}$ & $7.82 \mathrm{E}-03$ & CLL_0026 \\
\hline V\$SRF_C & $\begin{array}{l}\text { Genes with promoter regions }[-2 \mathrm{~kb}, 2 \mathrm{~kb}] \text { around transcription } \\
\text { start site containing the motif DCCWTATATGGNCWN which } \\
\text { matches annotation for SRF: serum response factor (c-fos serum } \\
\text { response element-binding transcription factor) }\end{array}$ & $1.16 \mathrm{E}-02$ & CLL_0026 \\
\hline V\$SRF_Q4 & $\begin{array}{l}\text { Genes with promoter regions }[-2 \mathrm{~kb}, 2 \mathrm{~kb}] \text { around transcription } \\
\text { start site containing the motif SCCAWATAWGGMNMNNNN } \\
\text { which matches annotation for SRF: serum response factor (c-fos } \\
\text { serum response element-binding transcription factor) }\end{array}$ & $1.16 \mathrm{E}-02$ & CLL_0026 \\
\hline PID_P38_GAMMA_DELTA_PATH & Signaling mediated by p38-gamma and p38-delta & $7.34 \mathrm{E}-03$ & $\mathrm{MCL} 0020$ \\
\hline
\end{tabular}




\begin{tabular}{|c|c|c|c|}
\hline WAY & & & \\
\hline CAGGTG_V\$E12_Q6 & $\begin{array}{c}\text { Genes with promoter regions [-2kb,2kb] around transcription } \\
\text { start site containing the motif CAGGTG which matches } \\
\text { annotation for TCF3: transcription factor } 3 \text { (E2A immunoglobulin } \\
\text { enhancer binding factors E12/E47) }\end{array}$ & $5.34 \mathrm{E}-06$ & MCL_0020 \\
\hline CTTTGT_V\$LEF1_Q2 & $\begin{array}{l}\text { Genes with promoter regions [-2kb,2kb] around transcription } \\
\text { start site containing the motif CTTTGT which matches } \\
\text { annotation for LEF1: lymphoid enhancer-binding factor } 1\end{array}$ & $1.52 \mathrm{E}-04$ & MCL_0020 \\
\hline TGGAAA_V\$NFAT_Q4_01 & $\begin{array}{c}\text { Genes with promoter regions }[-2 \mathrm{~kb}, 2 \mathrm{~kb}] \text { around transcription } \\
\text { start site containing the motif TGGAAA which matches } \\
\text { annotation for NFAT<br> NFATC }\end{array}$ & $8.18 \mathrm{E}-04$ & MCL_0020 \\
\hline CTTTGA_V\$SLF1_Q2 & $\begin{array}{c}\text { Genes with promoter regions [-2kb,2kb] around transcription } \\
\text { start site containing the motif CTTTGA which matches } \\
\text { annotation for LEF1: lymphoid enhancer-binding factor } 1\end{array}$ & $2.09 \mathrm{E}-03$ & MCL_0020 \\
\hline GGGAGGRR_V\$MAZ_Q6 & $\begin{array}{l}\text { Genes with promoter regions [- } 2 \mathrm{~kb}, 2 \mathrm{~kb}] \text { around transcription } \\
\text { start site containing the motif GGGAGGRR which matches } \\
\text { annotation for MAZ: MYC-associated zinc finger protein (purine- } \\
\text { binding transcription factor) }\end{array}$ & $5.71 \mathrm{E}-03$ & MCL_0020 \\
\hline GCANCTGNY_V\$MYOD_Q6 & $\begin{array}{c}\text { Genes with promoter regions [-2kb,2kb] around transcription } \\
\text { start site containing the motif GCANCTGNY which matches } \\
\text { annotation for MYOD1: myogenic differentiation } 1\end{array}$ & $5.71 \mathrm{E}-03$ & MCL_0020 \\
\hline TGANTCA_V\$AP1_C & $\begin{array}{c}\text { Genes with promoter regions [-2kb,2kb] around transcription } \\
\text { start site containing the motif TGANTCA which matches } \\
\text { annotation for JUN: jun oncogene }\end{array}$ & 7.14E-03 & MCL_0020 \\
\hline V\$HNF3B_01 & $\begin{array}{l}\text { Genes with promoter regions [-2kb,2kb] around transcription } \\
\text { start site containing the motif KGNANTRTTTRYTTW which } \\
\text { matches annotation for FOXA2: forkhead box A2 }\end{array}$ & 7.14E-03 & MCL_0020 \\
\hline V\$HNF6_Q6 & $\begin{array}{l}\text { Genes with promoter regions [-2kb,2kb] around transcription } \\
\text { start site containing the motif HWAAATCAATAW which matches } \\
\text { annotation for ONECUT1: one cut domain, family member } 1\end{array}$ & 8.67E-03 & MCL_0020 \\
\hline V\$HNF1_01 & $\begin{array}{l}\text { Genes with promoter regions [-2kb,2kb] around transcription } \\
\text { start site containing the motif GGTTAATNWTTAMCN which } \\
\text { matches annotation for TCF1: transcription factor 1, hepatic; LF- } \\
\text { B1, hepatic nuclear factor (HNF1), albumin proximal factor }\end{array}$ & $9.71 E-03$ & MCL_0020 \\
\hline
\end{tabular}

We next analyzed samples from the two CLL patients. As stated previously,

when we used the cleaved PARP assay results, we observed that CLL0021 was one of the most resistant to ibrutinib treatment, was resistant to ABT-199 treatment, and was among the least responsive to the combination. CLL0026, too, was extremely resistant to ibrutinib treatment alone, but was somewhat responsive to ABT-199 treatment and to the combination treatment.

These responses are also evident in the gene expression analysis of the patient samples' responses to the single and combination treatments. Despite the significant response of CLL0021 to combination ABT-199 and ibrutinib treatment, no significant transcriptional responses to the combination were observed. The genes affected by treatment were extremely limited as well; only 18 genes and 3 
genes, respectively, were affected by ABT-199 and ibrutinib treatment. In contrast, CLL0026 demonstrated a significant transcriptional response to the combination treatment. Indeed this was the only gene list for the CLL patient samples that allowed for a pathway and transcription factor analysis. The vast majority of the pathways implicated by this combination response are associated with immune system responses, including interleukin signaling and T cell signaling. However, p53 signaling, HIV induced apoptosis, caspase mediated apoptosis, T cell apoptosis, and the granzyme A mediated apoptosis pathway are all present in the list of pathways induced in CLL0026 by the combination treatment. The vast majority of these apoptosis associated pathways appear to contain CDKN1A, granzyme A, and caspase 8. All three of these genes are upregulated by a $\log 2$ fold change of 1.5 or better. These findings suggest that the upregulation of these proteins in response to the combination treatment is what drives the apoptotic response we observe in CLL0026.

Given the apoptotic responses for these patient samples, the results of the gene expression analysis are expected. MCL0020 shows the strongest apoptotic response to the combination in the cleaved PARP assay, and shows robust differential gene expression. The CLL patient samples demonstrated resistance to treatment given the lower levels of cleaved PARP, and the weaker differential gene expression corroborates this. The pathway enrichment that we observe for the differential gene expression shows there are responses observed in both the patient samples and cell lines, which indicates the efficacy of the combination and its mechanism of action is consistent between cell lines and patient samples. 


\subsection{DISCUSSION}

\subsubsection{Distal combinations induce unique patterns of response in individual}

cell lines and induce emergent responses

In our second transcriptional study of melanoma, we examined the combination of sorafenib and diclofenac. As we saw with the lines treated with PLX4720 and lapatinib or masitinib, each of the lines we assay exhibited unique transcriptional responses to the combination. However, we see very few potentiative responses, but rather the combination induces gene expression changes that do not appear in response to either drug alone. These emergent responses might be dependent on the very distal nature of the pathway targets of sorafenib and diclofenac. Like PLX4720, sorafenib targets the MAPK pathway by inhibiting multiple kinases associated with that pathway, and as mentioned early, we determined via compound substitution that diclofenac appears to be targeting Cox1 and Cox2. Unlike the interplay between the MAPK pathway and the ErbB signaling pathway, the interactions between these two pathways are not well studied. As a result, it is unclear as to the mechanism by which the combination induces these emergent responses to cause apoptosis. The combination of sorafenib and diclofenac is also effective across multiple melanoma drivers. It is well documented that PLX4720 is effective only in BRAFV600E melanomas (Halaban et al., 2010; Poulikakos et al., 2010, 2011; Søndergaard et al., 2010), and that it can activate melanomas lacking this genetic lesion. We observed this ourselves in multiple assays, where we see activation of the MAPK pathway in response to PLX4720 treatment for melanomas with BRAFWT. This makes sorafenib and 
diclofenac a very attractive combination for adaptation in the clinic, as it does not appear to be dependent on the genomic status of the melanoma.

Our results of the MCL screen strongly argue for the apparent superior effects of combinations that target distal rather than proximal targets in MCL. In our MCL panel, distal combinations vastly outperformed proximal combinations in terms of the Bliss difference. We observed that the lines demonstrated greater synergy to combinations that targeted distal pathways rather than proximal pathways; the combination of ibrutinib and ABT-199 vastly outperforming any of the combinations that targeted downstream targets of the BCR signaling pathway. In melanomas, we observed that the distal combination of sorafenib and diclofenac demonstrated synergy in multiple types of melanoma. However, it is unclear as to whether we have assayed enough lines to fully evaluate the efficacy of distal and proximal drug combinations. In our screens, we tend to have more proximal combinations than distal.

In our melanoma and B-Cell malignancy studies in this chapter, we do not observe the robust adaptive response to primary drug therapy we observed in $\mathrm{BRAF}^{\mathrm{V} 600 \mathrm{E}}$ melanomas treated with PLX4720. This is an indication that neither sorafenib treatment in melanoma nor ibrutinib treatment in B-Cell malignancies leverage pathway reactivation in response to treatment. Given the nature of the distal nature of the more successful secondary drugs in the MCL screen, there is a strong argument that resistance to ibrutinib therapy utilizes pathway bypass rather than pathway reactivation. 
In a recent paper dealing with treatment of lines with combination therapy and the interactions between single and combination treatments (Cappuccio et al., 2015), the authors postulate that there are a limited number of patterns of response to single and combination treatments, and argue for the idea of positive synergy, and emergent positive synergy, whose definitions match our interpretations of potentiative and emergent responses, respectively. This is evidence that we are not the only group observing these responses. However, we are the first to link these types of responses to the nature of the targets of the drugs.

\subsection{METHODS}

\subsubsection{Cell culture and cytotoxicity data generation}

\subsubsection{Melanoma cell culture}

SKMEL2, SKMEL24, SKMEL28 cells (American Type Culture Collection), A375, HT144, VMM5A, VMM39, VMM17, VMM18, VMM12, VMM15, DM13, DM6 DM122, DM331 (kind gift from Dr. Craig Slingluff, University of Virginia, Charlottesville, VA), and SLM2 (kind gift from Dr. Angela Zarling, University of Virginia) were propagated in RPMI-1640 (Invitrogen) supplemented with 5\% or 10\% FBS (Gemini Bio-Products). All melanoma cultures were maintained in a humidified chamber at $37^{\circ} \mathrm{C}$ with $5 \% \mathrm{CO}_{2}$. Inhibitors were obtained from the following sources: Lapatinib (LC Laboratories), 5-Aza-2'-deoxycytidine (decitabine) (Sigma). PLX4720 was a gift from Plexxikon.

\subsubsection{MCL cell culture}

JVM2, Z138, Jeko-1 and Mino cells were obtained from ATCC. JVM2 cells were cultured in RPMI 1640 (Invitrogen, Carlsbad, CA, USA) with 10 mM HEPES 
(Invitrogen), $1 \mathrm{mM}$ sodium pyruvate (Invitrogen), and 10\% FBS (Gemini, Sacramento, CA, USA). Z138 cells were cultured in Iscove's medium (Invitrogen) with $10 \%$ horse serum. Jeko-1 and Mino cells were cultured in RPMI 1640 (Invitrogen) with 15\% FBS (Invitrogen). All small molecule inhibitors for the initial screen were purchased from Selleck (Houston, TX, USA), with the exceptions of ABT199 (Active Biochemicals, Hong Kong, China), PD325901 (Pfizer, La Jolla, CA, USA), and SC-514 (EMD Biosciences). The cleaved PARP-FITC antibody and Annexin V / PI staining kit used for flow cytometry was from BD Biosciences (San Jose, CA, USA). MTS proliferation kit was purchased from Promega (Madison, WI, USA).

\subsubsection{Patient sample generation}

Specimens of peripheral blood or bone marrow aspirates were obtained from patients with MCL or CLL after obtaining informed consent in heparinized tubes. This project was approved by the Institutional Review Board at The University of Virginia. Peripheral blood mononucleated cells were isolated by the Biorepository and Tissue Research Facility. Cells were cultured in the following media: RPMI (Life Technology) with $10 \%$ fetal calf serum (Hyclone), 100mM HEPES (Life Technology), Non-essential amino acids (Life Technology), IL2 (Peprotech) and CpG (Invivogen). The tumor cells were immediately treated in vitro with the specific drugs.

\subsubsection{Cytotoxicity assays}

Four hours after being plated in 96-well plates, cells were treated with inhibitors or vehicle control in phenol red-free RPMI-1640 (Invitrogen) without FBS and incubated for 3 days at $37^{\circ} \mathrm{C}$. alamarBlue (Invitrogen) was added to wells and incubated for 4 hours at $37^{\circ} \mathrm{C}$. Fluorescence was measured at $560 \mathrm{~nm}$ 
excitation/590 nm emission on a Synergy 2 plate reader (BioTek Instruments). Mean results and SE were calculated for triplicate samples.

\subsubsection{Flow Cytometric analyses:}

Cleaved PARP: Cells were plated at a density of $1 \times 10^{6}$ cells/well (JVM2) and $8 \times 10^{5}$ cells/well (Z138) in 6 well plates. Cells were treated for 72 hours as described in the text, and then stained as previously described using cleaved PARP FITC antibody.Annexin V/PI: $1 \times 10^{5}$ pretreated cells were washed with cold phosphatebuffered saline (PBS) and stained with an Annexin VITC and propidium iodide in accordance with the manufacture's procedure (BD PharMingen, San Diego, CA, USA). Cells were assayed using a FACScantoll flow cytometer system (BD Biosciences) interfaced to FACS Diva software (v 6.0), and analyzed with Flow Jo (v7.2.1).

\subsubsection{Generation of transcriptome, proteome, DNA methylome and exome data}

\subsubsection{Gene array}

Cells were plated and incubated overnight before being treated, in duplicate, with inhibitors or vehicle control in phenol red-free RPMI-1640 without FBS for 8 hours at $37^{\circ} \mathrm{C}$. Cells were placed on ice and rinsed with cold $1 \times$ PBS. Cells were collected and RNA was isolated using the Qiashredder (Qiagen) and RNeasy Mini Kit (Qiagen). RNA was quantified on the NanoDrop 2000 spectrophotometer (Thermo Scientific) and RNA quality was inspected on a 1\% agarose gel. Biotin labeled RNA was hybridized to Illumina 3'IVT human HT-12 BeadChip arrays.

\subsubsection{Reverse Phase Protein Array}

Cells were plated in p60 dishes and incubated overnight to allow the cells to adhere before being treated with inhibitors or vehicle control in phenol red-free 
RPMI Medium 1640 without fetal bovine serum for 1, 8, or 24 hours. Cells were washed and lysed in 1:12x Sample Buffer:Tissue Extraction Reagent (T-PER) (Life Technologies). Following lysis the samples were sonicated and centrifuged to clear. Samples were diluted to requested concentration and sent to the lab of Emanuel (Chip) Petricoin at George Mason University for Reverse Phase Array Analysis.

\subsubsection{Statistical Analysis of Functional Genomics}

Illumina microarray data was variance stabilized transformed (Federici et al., 2013; Improta et al., 2011) using the lumi Bioconductor package in R (Du et al., 2008). Significantly varying genes and cell lines were clustered using the R package pvclust (Suzuki and Shimodaira, 2006) with the Pearson correlation distance measure and average linkage. We identified genes whose expression was significantly altered for each cell lines in response to treatment using limma to perform moderated t-tests and applying a 1\% FDR cutoff. Illumina DNA methylation array data underwent normalization using SWAN and was logit transformed using minfi as described here (Aryee et al., 2014). A moderated t-test was performed as above to assay for differentially methylated loci in response to treatment. Normalized $\log 2$ reverse phase protein array (RPPA) data was generated using methods described in (Improta et al., 2011). We performed a moderated t-test analysis described above (for gene expression data) and found a number of differentially phosphorylated sites at a FDR of 1\%.

\subsubsection{Pathway Enrichment Analysis}

Gene sets found to be significant underwent pathway enrichment analysis via Pathway Express and Gene Set Enrichment Analysis (GSEA) using MSigDB datasets. 
Significant gene sets and pathways were identified as having a FDR enrichment value of 0.05 or less for both tools.

\subsection{ACKNOWLEDGMENTS}

Sections of this chapter were adapted from Combinatorial drug screening and molecular profiling reveal diverse mechanisms of intrinsic and adaptive resistance to BRAF inhibition in V600E BRAF mutant melanomas (Roller et al., 2016), Synthetic lethal screening with small-molecule inhibitors provides a pathway to rational combination therapies for melanoma (Roller et al., 2012), and Combinatorial drug screening identifies synergistic co-targeting of Bruton's tyrosine kinase and the proteasome in mantle cell lymphoma (Axelrod et al., 2014a). 


\section{Future Directions}

\subsection{Combination therapy can overcome resistance to targeted monotherapy in melanoma, chronic lymphocytic leukemia, and mantle cell lymphoma}

Our work has reiterated a known flaw in the use of targeted monotherapies: rapid emergence of resistance or its presence even at the outset. In BRAFV600E melanoma, we observed that inhibiting mutant BRaf with PLX4720 induces a strong adaptive response via the ErbB signaling pathway, leading to the activation of the PI3K pathway and the reactivation of the MAPK pathway. Across multiple drug screens using a synthetic lethal paradigm, we have identified a number of combinations that overcome resistance, both acquired and intrinsic, in a synergistic manner. Again, in our screen of $\mathrm{BRAF}^{\mathrm{V} 600 \mathrm{E}}$ melanoma, we determined that targeting the ErbB signaling pathway blocked the adaptive response, allowing the lines to respond to the therapy.

For a subset of these combinations, we have identified potential mechanisms of resistance to the monotherapies, as well as potential mechanisms of synergistic response to the combinations. We have developed pipelines for analysis, in parallel, of gene expression, DNA methylation, and protein levels and posttranslational modifications, and identified methods of synthesizing the results, across the multiple datasets, into a coherent result.

In the case of melanoma (Chapter 2), we confirmed that PLX4720 treatment induces compensatory signaling that can lead to further cell growth. Other groups have identified this adaptive signaling response (Abel et al., 2013; Lito et al., 2012, 
2013), but we observe it at a systems level. We were able to determine that this response was likely mediated by a transcription factor network that contains MAPK pathway phosphorylation sites, indicating that the inhibition of the pathway induces an adaptive growth response dependent on downstream targets of the pathway (Section 2.3.5). Among the pathways that this group of transcription factors targets is the ErbB signaling pathway, which explains why inhibiting RTKs in combination with Raf inhibition works synergistically in melanomas and gives insight as to mechanisms of other Raf driven cancers that are not responsive to Raf inhibitor monotherapy: Raf inhibition induces RTK activation; RTK inhibition prevents the adaptive response (Section 2.3.5; Section 2.3.6).

In our studies of B-cell malignancies (Chapter 4), specifically mantle cell lymphoma and chronic lymphocytic leukemia, we again observe that combinations are an effective means for overcoming intrinsic resistance. Additionally, we were able to demonstrate the effectiveness of the combinations not only in cell lines, but in patient samples as well (Section 4.3.8). Both MCL and CLL can be driven by the overactivation of the BCR pathway, leading to growth and pro survival pathways. Ibrutinib, an inhibitor of BTK, serves to limit the ability of MCL and CLL to drive those pathways. However, in our cell lines and patient samples we observe varying levels of resistance to ibrutinib monotherapy. When we performed our synthetic lethal screen of drug combinations in MCL lines, we observed that combining ibrutinib with ABT-199 provides by far the most effective result and that the effectiveness of the combinations as measured by synergy score is somewhat dependent on the distance between the targets of the drugs. Some combinations of 
drugs that repeatedly targeted the BCR pathway performed worse than combinations including drugs that targeted proteins tangentially connected to the BCR pathway, such as was the case of ibrutinib combined with ABT-199 (Section 4.3.2).

\subsection{The adaptive response to primary drugs reveals potential targets for secondary drug treatment}

In our BRAFV600E melanoma screen, we identified three secondary drugs that resulted in synergistic effects when combined with PLX4720: OSU-03012, an inhibitor of PDK1, but has been observed to have other targets (Booth et al., 2012); lapatinib, an inhibitor of ErbB family members; and masitinib, an inhibitor of PDGFR, VEGFR and KIT (Section 3.3.1). The targets of these three drugs are all upstream activators or known members of the PI3K signaling pathway. Like the MAPK pathway, the PI3K pathway is responsible for driving growth and survival (Carnero et al., 2008; McCubrey et al., 2006).

Treating our panel of BRAFV600E melanomas with PLX4720 revealed that ErbB signaling was activated in lines resistant to the treatment (Section 2.3.5); our combination screen demonstrated the effectiveness of targeting ErbB signaling as a means to synergistically kill cancer cell lines that are resistant to monotherapy (Section 3.3.1). When we looked for other pathways that are activated by Raf inhibition treatment, we observed that noncanonical MAPK signaling, noncanonical WNT signaling, and PI3K signaling pathways are induced (Section 2.3.5). Given that we know blocking the ErbB adaptive response yields synergy, it would be 
interesting to determine whether inhibitors that target these alternative adaptive responses would also elicit synergistic responses. In our BRAFV600E melanoma screen (Section 3.3.1), we did observe that inhibition of the PI3K signaling pathway was effective at inducing synergy in a subset of the cell lines.

Clinical trials have been performed using inhibitors that target BRaf and MEK in combination to help limit the reactivation of the MAPK pathway (Flaherty et al., 2012b), and this combination is now the standard of care. Our results suggest that a more effective strategy would be to inhibit targets that engage parallel growth pathways e.g. RTKs (Section 3.3.1). Further combination studies could be done to determine whether this hypothesis holds true under clinical scrutiny. Additionally, the combination of BRaf and MEK inhibition appears to be effective only in patients who have not yet been exposed to targeted BRaf inhibition alone (Flaherty et al., 2012b). This would seem to be further evidence that it may be more effective to target parallel pathways that are a part of the adaptive response.

Regarding gene expression analysis, we have not yet investigated the adaptive responses to sorafenib in our pan melanoma study (Section 4.3.1), or ibrutinib in our B-cell malignancy study (Section 4.3.2). However, given the combinations that we have identified in the various screens, we have the capability to focus our analysis on the pathways targeted by the secondary drug. In our study of sorafenib and diclofenac, our protein substitution studies revealed that sorafenib is acting at least in part on MAP Kinase signaling, and diclofenac is inhibiting cyclooxygenase signaling (Section 4.3.1). Cyclooxygenases are proteins that have been widely implicated in many cancers, but their exact role is still under 
investigation. Cyclooxygenases may function significantly in the inhibition of apoptosis and immune surveillance, the promotion of angiogenesis, the elevation of cancer invasiveness and metastasis, and the modulation of cell differentiation (Roller et al., 2012). Lack of detailed knowledge of cyclooxygenase functions in cancer makes it unclear as to what adaptive response we should be looking for in the case of sorafenib treatment. However, we can use knowledge of the pathways induced by Raf inhibition from our studies in PLX4720 to help connect the specific mechanisms or downstream effects of cyclooxygenase that are being affected by diclofenac treatment.

As previously mentioned, the most effective combination for the MCL screen was ABT-199 combined with ibrutinib (Section 4.3.2). It has been observed that the BCR signaling pathway does interact tangentially with the apoptosis pathway (Saba and Wiestner, 2014). ABT-199 targets BCL2, a prominent member of the apoptotic program, and manipulation of BCL2 activity has been shown to strongly influence the tendency of a cell to either evade or enter apoptosis. Again, we have not yet investigated the mechanism or mechanisms by which an adaptive response to ibrutinib could be ablated by ABT-199 treatment, but given the role of BCL2 in apoptosis, it is possible that we are reinforcing apoptotic signaling.

Given our work in BRAFV600E melanoma, it becomes apparent that more exploration of adaptive responses is required to accurately enumerate mechanisms of resistance to monotherapy and synergistic response to combinations. Future work will require the isolation of these adaptive responses by functional genomic assays and evaluation of these hypotheses by biochemical follow ups, such as 
western blots and RT-PCR. Identifying effective combinations will alone provide a wealth of results for clinicians to act upon.

\subsection{Integrative analysis revealed significant insights into the adaptive response in melanoma}

Initial analysis of the gene expression response to PLX4720 in BRAFV600E melanoma was unable to identify differential responses to treatment based on established phenotypes. Lines demonstrating synergy did not appear to respond any differently to the treatment than lines that did not demonstrate synergy. Nor did stratifying the cell lines based upon the basal transcriptome (Section 2.3.2) or the basal methylome (Section 3.3.4) reveal significant insights. While we were able to determine that a subset of our lines contained higher expression of MITF, a master regulator for melanocyte lineage (Garraway et al., 2005), the dynamic range of this variation was much lower than what other groups had reported (Konieczkowski et al., 2014; Müller et al., 2014), and thus was insufficient to be a useful predictor in our studies. Attempting to segregate the lines based on the zygosity status of the V600E allele also did not serve as a means to predict which lines would respond in a synergistic manner to the combination (Section 2.3.4).

Reclassifying the lines using unsupervised hierarchical clustering of the lines' responses to single and combination treatments allowed five groups of cell lines to be classified according to cytotoxic responses. Using these five cytotoxicity groups, we were able to identify a number of differential responses in the transcriptome and 
proteome, and to explain the stratifications of the lines at a basal state in the transcriptome and exome (Section 2.3.5).

It cannot be stressed enough that appropriate classification of the lines was integral to effectively evaluating and arriving at hypotheses for validation. Our differential analyses at the cell line level revealed very little information about the adaptive response. It was only by combining the lines from the point of view of their cytotoxic responses that we were able to observe the compensatory pathway of ErbB signaling, and identify the additional adaptive responses in WNT, PI3K, and the noncanonical MAPK signaling pathways (Section 2.3.5).

Integrative analysis was also essential to validating our hypothesis regarding the mechanism of synergy for the combination of PLX4720 and lapatinib. Fold changes induced by combination treatment in each line affected completely different sets of genes. Very few genes were induced in multiple lines. Taking each set of differentially expressed genes through a pathway enrichment analysis revealed that the lines responding synergistically were all leveraging ErbB signaling, PI3K signaling, and p53 signaling (Section 3.3.2). These pathways are a strong indication that the combination therapy prevented the adaptive response, as evidenced by their enrichments for all of the lines that demonstrate synergy.

We also observed the adaptive response and its inhibition in our RPPA study on SKMEL24 and VMM5A. Here we observed a knockdown of phospho AKT at 1 hour after lapatinib treatment, which was maintained at 8 hours. By 24 hours, levels of AKT phosphorylation had returned to approximately basal levels. Under 
treatment with the combination, however, the initial knockdown persisted for the duration of the study (Section 3.3.3). These observations show that adaptive responses can be effectively overcome by combinatorial therapies.

Examining the mechanisms of DM331 resistance and synergy also benefited from integrative analysis. DM331 and A375 share nearly identical genomic (Section 2.3.4), transcriptomic (Section 2.3.2), and methylomic states (Section 3.3.5). Their responses to PLX4720 treatment show no similarities. This is unfortunate, as it demonstrates that response to treatment may not be identifiable in the basal state. We observe a similar result when we examine the PCA plot of the BRAFV600E melanomas (Section 2.3.2). There we observed that although the CGC and CGD groups have very little separation along the first principal component they exhibit very different responses to the combination.

Examining the methylome in DM331 after treatment with lapatinib alone, PLX4720 alone, or the combination, revealed no significant changes in methylation levels at any loci (Section 3.3.5). However, we observed that globally knocking down methylation induced sensitivity to lapatinib in DM331, but had no effect on A375. Pursuing the effects of this treatment with decitabine on the transcriptome revealed that DM331 induces a similar transcriptional response to the one we observe in CGC in response to PLX4720 treatment. This is a confusing result, as it indicates that either the adaptive response is dependent on changes in DNA methylation or that lines have a limited ability to adapt to drug treatments. If the adaptive response were dependent on DNA methylation, we might have expected changes in methylation when treated with the drugs alone, but such changes were not 
observed. If cells have a limited ability to adapt, then we would expect lines resistant to BRAF inhibition to respond to higher doses of BRAF inhibition, or to BRAF and MEK inhibition, but again this appears not to be the case.

\subsection{Emergent and potentiative combination responses appear dependent on proximity of drug targets}

In our exploration of responses to combinations across melanoma, CLL and MCL, we observed a rather interesting phenomenon in the gene expression data. In $\mathrm{BRAF}^{\mathrm{V} 600 \mathrm{E}}$ melanoma, lines exposed to the combination of PLX4720 and lapatinib showed a pattern of gene expression responses to the combination that was an amplification or potentiation of the response to PLX4720 treatment alone; lapatinib treatment alone elicited very minimal effects. Examination of the pathways targeted by this combination revealed that ErbB family members, the targets of lapatinib, can lead to the activation of BRAF, the target of PLX4720. We suspect that, due to the proximal nature of these two targets, inhibiting both will lead to an amplified inhibition of the downstream targets of both these pathways, which could explain the apparent amplification in responses at the gene expression level. That amplification could also be entirely dependent on the mechanism of action. PLX4720 treatment induces ErbB signaling, and blocking that adaptive signaling increases the inhibitory response (Section 3.3.2). We acknowledge that data on drug combination responses are more limited than data on single drug treatments, so evaluating the mechanisms of a proximal potentiating response remains preliminary. 
In the other combinations we examined (sorafenib and diclofenac in multiple types of melanomas, and Ibrutinib and ABT-199 in MCL), we observed emergent responses: substantial responses to the combination were observed where no responses had been observed after the single drug treatments. We were able to determine, in the case of the sorafenib and diclofenac combination treatment, that we were targeting MAP Kinase pathway and cyclooxygenase signaling, respectively; it has been observed that cyclooxygenases can induce Raf signaling via a PGE1-dependent signaling cascade (Section 4.3.1). In the case of the MCL study, ibrutinib targets BTK, and ABT-199 targets BCL-2. These targets have very distal associations so far as we are aware. However, again we observed an emergent response to the combination: genes that showed no response to either drug now having strong responses to the blockade in combination (Section 4.3.4).

\subsection{Combinations composed of distal drug pairs may perform better than combinations composed of proximal drug pairs}

In addition to observing variations in response due to proximal and distal drug pairs, we observed in MCL that distal drug pairs performed better than proximal drug pairs. Likewise, in melanoma, a distal drug pair was able to demonstrate synergy regardless of driver mutation status. These two examples would seem to argue that distal drug pairs can be more effective than proximal drug pairs.

In the case of MCL, the primary driver is the overactivation of the BCR pathway (Saba and Wiestner, 2014; Swerdlow and Williams, 2002). Feedback 
inhibition of the BCR pathway has not been observed as part of the adaptive response in MCL (Axelrod et al., 2014a), in contrast to observations of melanoma. In melanoma, it is well documented that feedback activation essentially turns the pathway back on after it has been inhibited. This reactivation is a strong component of the melanoma's robust adaptive response to BRAF inhibition.

As previously discussed, distal drug combinations tend to induce emergent responses; proximal drug combinations tend to induce potentiative drug responses. Generally, it is currently unclear as to how these patterns relate to cellular phenotypes. Again, given the results of our MCL and melanoma screens, we suggest that emergent responses are more desirable. It is our hypothesis that emergent responses do not depend strongly on adaptive responses or feedback loops for their effectiveness, making it more difficult for the cells to overcome the combination. Intriguingly, the emergent combinations for MCL and melanoma both converged on either downregulating survival genes/pathways, or upregulating apoptosis genes/pathways. Targeting distal pathways that cancer cells depend on for growth and survival may result in acute cellular stress and apoptosis. Much more work must be done to test this hypothesis.

Clinical trials of combined BRAF and MEK inhibition in melanoma indicate that the combination is not effective in patients who are already resistant to BRAF monotherapy (Flaherty et al., 2012b). We have not yet examined transcriptional profiles of this combination, but MEK is downstream of BRAF, and we think the responses here would mirror our work in MCL with ibrutinib combined with 
idelalisib, another combination that targets two nodes in the same pathway (Axelrod et al., 2014a).

\subsection{Transcription factor enrichment analysis reveals a strong role for ETS family members in the adaptive response}

Among our analyses we saw the emergence of a transcription factor network in response to PLX4720 treatment in CGC (Section 2.3.5), and in response to decitabine treatment in DM331 (Section 3.3.7). In both of these cases we saw the induction of the ErbB signaling pathway, and a sensitization to lapatinib treatment. It is unclear why a BRaf inhibitor would activate a battery of responses similar to those activated by global DNA methylation knockdown. It is even more confounding, given that treatment with PLX4720, lapatinib, or the combination, did not induce any noticeable methylation changes in DM331. Ideally, we would profile these potential transcription factor binding sites with ChIP Seq experiments to determine the true occupancy of these factors, both at a basal level, and in response to single and combination treatment. It should be noted that the tool we use for transcription factor enrichment analysis, MSigDB (Subramanian et al., 2005), may be biased towards specific motifs, which could be driving our observation that these transcription factors are enriched after two different treatments in melanoma.

The expectation is that in BRaf mutant melanomas there is an extremely high basal level of MAPK activity due to the mutant BRaf kinase. This persistent activity floods the cells with negative feedback regulators of the pathway, including factors that limit the activity of the pathway's downstream transcription factors. With 
inhibition of mutant BRaf, these negative feedback mechanisms become attenuated, allowing for the release of inhibition. The transcription factors are now free to act, and induce the activation of ErbB and other alternative growth pathways, such as PI3K. As a result of this induction, lapatinib is now effective at shutting down this parallel pathway, leading to the observed synergistic cytotoxicity.

Being able to effectively and accurately identify the transcription factors mediating the adaptive response would allow for better combination therapies. At present, focus on transcription factors as drug targets is extremely dubious. First of all, because they lack enzyme activity and function by protein-protein and proteinDNA binding, they until recently have been considered "undruggable." In addition, transcription factors are ubiquitous across many cell types; inhibiting them can lead to excessive and insurmountable side effects. If it were possible to identify the transcription factors that mediate a particular adaptive response, it might become possible to target the negative regulator of the factor, such as the kinase or phosphatase responsible for releasing the transcription factor from inhibition. These would be much more appealing targets for inhibition.

\subsection{Observed adaptive responses to secondary drugs are blocked due to combination treatment}

In our RPPA analysis of PLX4720, lapatinib, and combination treatment of VMM5A and SKMEL24, we observe a case of a secondary drug inducing an adaptive response (Section 3.3.3). We determined that phospho AKT compensates its down regulation by lapatinib treatment, but under combination treatment the lines no 
longer demonstrate the adaptive response. Throughout this study, our approach to adaptive responses followed from the hypothesis that the primary drug induces a response and the secondary drug targets this response. The RPPA study shows that this is a simplification of the true complexities of the situation.

In our MCL screen, we see ABT-199, as a secondary drug, having significant effects on responses to treatment (Section 4.3.2). Both ibrutinib and ABT-199 are extremely effective monotherapies, but for the purposes of this study, we focused on ibrutinib. It is clear that ABT-199 warrants further consideration as a primary drug.

In melanoma, we ran across repeated examples of RTK inhibitors performing synergistically with MAPK pathway inhibition (3.3.1). It is clear that RTK inhibition is a very effective means of cancer therapy, as is evidenced by its widespread use against other cancers. Recent studies show that pan RTK inhibition is substantially more effective than combining up to six targeted therapies (Langdon et al., 2015).

Taken together, these observations indicate that many secondary drugs warrant further exploration as primary drugs. For all of our studies, we chose specific primary drugs, and combined them with a broad panel of secondary drugs. It would be beneficial to design screens where all drugs are treated as members of combination without labeling them as secondary or primary, especially as the library of inhibitors becomes broader. 


\subsection{Leveraging phenocopying observed in previous screens could result in better screen designs}

In our melanoma screen, we had a substantial number of secondary drugs that targeted the same pathway at different levels (Section 3.3.1). This strategy might no longer be ideal. In our melanoma studies, we considered a high number of synergistic combinations that contained secondary drugs targeting the same pathways. If the goal is to identify novel combinations for treatment, future screens ought to cover as many pathways as possible.

We observed lines in which a particular pathway inhibition was not effective, but another one was. This would seem to indicate that there is an optimum level of redundancy for discovering new combinations. It remains unclear whether our study was too redundant, and/or whether we missed promising combinations because we did not have an appropriate inhibitor for a specific cell line (Section 3.3.1).

A better screen design might involve a multilevel screening strategy. A high level screen would seek to use promiscuous drugs that target pathways very broadly in combination. Any combinations that demonstrate significant synergy could then be further investigated using more targeted compounds. Effectively, this would allow for high-throughput screening of pathway inhibition, followed by screening of specific mechanisms of synergy. 


\subsection{Computational integration of data could reveal additional specific mechanisms of resistance and synergy}

PARADIGM is a computational tool designed to integrate functional genomics data and estimate the activities of individual pathways (Vaske et al., 2010). Throughout our studies, we have integrated datasets in order to elucidate the biology underlying the responses we are observing. However, we are limited in our ability to truly combine the interactions of the different datasets. PARADIGM is one of many tools that currently exist to integrate and extrapolate data.

The adaptive response to PLX4720 treatment has been widely reported and confirmed in the melanoma literature (Kugel et al., 2014). The question is, what other pathways and targets remain unknown, and whether computational integration would reveal such novel pathways of interest and new targets. Screening is a very practical approach to understanding mechanisms of synergy and resistance. Limiting one's screen to FDA approved compounds allows for quick iteration through combinations and advancement to clinical trials. However, in melanoma we observed, in addition to ErbB pathway activation post PLX4720 treatment, activation of noncanonical Wnt and noncanonical MAPK signaling (Section 2.3.5). It would be ideal to determine what roles these pathways play in the adaptive response, and whether targeting them with drugs would be more or less effective than targeting ErbB signaling. 


\subsection{Future analyses}

The melanoma and the MCL and CLL screening projects both produced a substantial amount of data. In the former, we assembled SNP chip data and gene expression data for a number of lines exposed to sorafenib and diclofenac. Further exploration of the sorafenib and diclofenac response in melanomas would allow for better characterization of the adaptive responses. In our first analysis, we limited our pathway enrichment analyses to a gene ontology enrichment analysis (Section 4.3.1). We were able to see that the combination induces the expression of many genes associated with apoptosis and cell survival. This result is lacking in description compared to our other analyses with pathway enrichments.

Additionally, DM331 is among the lines we treated with sorafenib and diclofenac. Given the unique nature of DM331 and the extent of data we have available on its responses to various perturbations, it would be interesting to determine if sorafenib also induces the adaptive response we see under PLX4720 and decitabine treatment.

It is also unclear as to what pathways are induced under sorafenib and diclofenac treatment. We did not observe a strong adaptive response to diclofenac treatment, therefore it is uncertain as to what the downstream effects of cyclooxygenase inhibition are. Lacking this information, it is difficult to determine

its role in the mechanism of synergy (Roller et al., 2012). Work is ongoing to determine mechanisms by which diclofenac is effective in cancer therapy (Pantziarka et al., 2016). It is possible that a deeper analysis of sorafenib and 
diclofenac responses might help elucidate the pathways in which cyclooxygenases play a critical role.

In addition to the sorafenib and diclofenac data, SNP chip data exists for 16 melanoma cell lines. These data would allow us to determine the copy number variation present in our melanoma samples. It is entirely possible that the effects we observe in regards to zygosity of the V600E allele in BRAF are more strongly associated with copy number. There are a number of sensitive heterozygous BRAF lines whose mechanism of sensitivity is completely unknown. It is possible that they have the dosage of the mutant allele could be contributing to this sensitivity. Because the adaptive response is dependent on WT BRAF binding with PLX4720 and another RAF monomer, any conditions that affect the binding event would modulate the adaptive response.

Another potential mechanism of resistance driven by copy number could be increases in RTKs. As discussed throughout, RTKs contribute significantly to resistance and adaptive responses of melanoma. Higher dosages of RTKs can make cells extremely sensitive to ligand binding. DM331 has extremely high basal levels of EGFR protein and transcript (Section 2.3.2). It is possible that it has copy number gains in EGFR. This would account for the robust resistance of the line to PLX4720 treatment, and its apparent lack of response. If the adaptive response is already turned on, it is not required to be further engaged.

MCL patient sample 20 presents a unique case to study the rise of in vivo resistance to ibrutinib monotherapy. MCL_0020 responded well to ibrutinib 
treatment initially, but then relapsed and became extremely resistant (Section 4.3.5). Each time the patient came into the clinic, blood samples were taken and stored. For the case of the ex vivo gene expression study, those cells were obtained after the patient had been treated with ibrutinib. Examined from this context, it would be extremely informative to examine the changes in gene expression and responses to treatments for each of the patient samples available to study.

\subsection{Conclusions}

In this document, we have demonstrated the efficacy of combination therapy at overcoming the adaptive response to monotherpy in resistant melanoma, and MCL and CLL. Treating melanomas with PLX4720 induced the expression of the ErbB signaling pathway. Treating melanomas with PLX4720 and lapatinib, an inhibitor of Erbb family members, ablated the response and led to synergistic cytotoxicity. MCL and CLL are B-cell malignancies with generally poor prognoses. Ibrutinib initially proved to be a miracle drug, but resistant patients soon came into clinics. Here, we have identified a number of secondary drugs, notably ABT-199, that synergistically and swiftly induce apoptosis in MCL cell lines and in MCL and CLL patient samples.

We demonstrated that integrative analysis of multiple datasets serves to inform results and interpretations. For example, combining exome data with methylome data for melanoma revealed that a mutation in DNMT3A was driving the strong separation in the basal methylation data. Pathway enrichment analysis of 
protein and gene expression data served to validate our hypothesis of compensatory signaling, and the mechanisms of synergy. 


\section{References}

Abel, E.V., Basile, K.J., Kugel, C.H., 3rd, Witkiewicz, A.K., Le, K., Amaravadi, R.K., Karakousis, G.C., Xu, X., Xu, W., Schuchter, L.M., et al. (2013). Melanoma adapts to RAF/MEK inhibitors through FOXD3-mediated upregulation of ERBB3. J. Clin. Invest. 123, 2155-2168.

Adzhubei, I.A., Schmidt, S., Peshkin, L., Ramensky, V.E., Gerasimova, A., Bork, P., Kondrashov, A.S., and Sunyaev, S.R. (2010). A method and server for predicting damaging missense mutations. Nat. Methods 7, 248-249.

Akbani, R., Akdemir, K.C., Aksoy, B.A., Albert, M., Ally, A., Amin, S.B., Arachchi, H., Arora, A., Auman, J.T., Ayala, B., et al. (2015). Genomic Classification of Cutaneous Melanoma. Cell 161, 1681-1696.

Alizadeh, A.A., Eisen, M.B., Davis, R.E., Ma, C., Lossos, I.S., Rosenwald, A., Boldrick, J.C., Sabet, H., Tran, T., Yu, X., et al. (2000). Distinct types of diffuse large B-cell lymphoma identified by gene expression profiling. Nature 403, 503-511.

Allison, D.B., Cui, X., Page, G.P., and Sabripour, M. (2006). Microarray data analysis: from disarray to consolidation and consensus. Nat. Rev. Genet. 7, 55-65.

Antony, R., Emery, C.M., Sawyer, A.M., and Garraway, L.A. (2013). C-RAF mutations confer resistance to RAF inhibitors. Cancer Res 73, 4840-4851.

de Araújo, É.S.S., Pramio, D.T., Kashiwabara, A.Y., Pennacchi, P.C., Maria-Engler, S.S., Achatz, M.I., Campos, A.H.J.F.M., Duprat, J.P., Rosenberg, C., Carraro, D.M., et al. (2015). DNA Methylation Levels of Melanoma Risk Genes Are Associated with Clinical Characteristics of Melanoma Patients. BioMed Res. Int. 2015, 376423.

Aryee, M.J., Jaffe, A.E., Corrada-Bravo, H., Ladd-Acosta, C., Feinberg, A.P., Hansen, K.D., and Irizarry, R.A. (2014). Minfi: a flexible and comprehensive Bioconductor package for the analysis of Infinium DNA methylation microarrays. Bioinformatics $30,1363-1369$.

Ashburner, M., Ball, C.A., Blake, J.A., Botstein, D., Butler, H., Cherry, J.M., Davis, A.P., Dolinski, K., Dwight, S.S., Eppig, J.T., et al. (2000). Gene ontology: tool for the unification of biology. The Gene Ontology Consortium. Nat. Genet. 25, 25-29.

Atefi, M., von Euw, E., Attar, N., Ng, C., Chu, C., Guo, D., Nazarian, R., Chmielowski, B., Glaspy, J.A., Comin-Anduix, B., et al. (2011). Reversing melanoma cross-resistance to BRAF and MEK inhibitors by co-targeting the AKT/mTOR pathway. PloS One 6, e28973. 
Atkinson, J.M., Rank, K.B., Zeng, Y., Capen, A., Yadav, V., Manro, J.R., Engler, T.A., and Chedid, M. (2015). Activating the Wnt/ $\beta$-Catenin Pathway for the Treatment of Melanoma--Application of LY2090314, a Novel Selective Inhibitor of Glycogen Synthase Kinase-3. PloS One 10, e0125028.

Axelrod, M., Ou, Z., Brett, L.K., Zhang, L., Lopez, E.R., Tamayo, A.T., Gordon, V., Ford, R.J., Williams, M.E., Pham, L.V., et al. (2014a). Combinatorial drug screening identifies synergistic co-targeting of Bruton's tyrosine kinase and the proteasome in mantle cell lymphoma. Leukemia 28, 407-410.

Axelrod, M.J., Gordon, V., Mendez, R.E., Leimgruber, S.S., Conaway, M.R., Sharlow, E.R., Jameson, M.J., Gioeli, D.G., and Weber, M.J. (2014c). p70S6 kinase is a critical node that integrates HER-family and PI3 kinase signaling networks. Cell. Signal. 26, 1627-1635.

Axelrod, M.J., Mendez, R.E., Khalil, A., Leimgruber, S.S., Sharlow, E.R., Capaldo, B., Conaway, M., Gioeli, D.G., Weber, M.J., and Jameson, M.J. (2014b). Synergistic apoptosis in head and neck squamous cell carcinoma cells by co-inhibition of insulin-like growth factor-1 receptor signaling and compensatory signaling pathways. Head Neck.

Balch, C.M., Gershenwald, J.E., Soong, S.-J., Thompson, J.F., Atkins, M.B., Byrd, D.R., Buzaid, A.C., Cochran, A.J., Coit, D.G., Ding, S., et al. (2009). Final version of 2009 AJCC melanoma staging and classification. J. Clin. Oncol. Off. J. Am. Soc. Clin. Oncol. 27, 6199-6206.

Barretina, J., Caponigro, G., Stransky, N., Venkatesan, K., Margolin, A.A., Kim, S., Wilson, C.J., Lehár, J., Kryukov, G.V., Sonkin, D., et al. (2012). The Cancer Cell Line Encyclopedia enables predictive modeling of anticancer drug sensitivity. Nature 483, 603-607.

Basu, A., Bodycombe, N.E., Cheah, J.H., Price, E.V., Liu, K., Schaefer, G.I., Ebright, R.Y., Stewart, M.L., Ito, D., Wang, S., et al. (2013). An interactive resource to identify cancer genetic and lineage dependencies targeted by small molecules. Cell 154, 1151-1161.

Bernal, A. (2001). Survival of leukemic B cells promoted by engagement of the antigen receptor. Blood 98, 3050-3057.

Bollag, G., Hirth, P., Tsai, J., Zhang, J., Ibrahim, P.N., Cho, H., Spevak, W., Zhang, C., Zhang, Y., Habets, G., et al. (2010). Clinical efficacy of a RAF inhibitor needs broad target blockade in BRAF-mutant melanoma. Nature 467, 596-599.

Booth, L., Cruickshanks, N., Ridder, T., Chen, C.-S., Grant, S., and Dent, P. (2012). OSU03012 interacts with lapatinib to kill brain cancer cells. Cancer Biol. Ther. 13, 15011511. 
Byrd, J.C., Furman, R.R., Coutre, S.E., Flinn, I.W., Burger, J.A., Blum, K.A., Grant, B., Sharman, J.P., Coleman, M., Wierda, W.G., et al. (2013). Targeting BTK with ibrutinib in relapsed chronic lymphocytic leukemia. N. Engl. J. Med. 369, 32-42.

Cancer Genome Atlas Research Network (2008). Comprehensive genomic characterization defines human glioblastoma genes and core pathways. Nature 455, 1061-1068.

Capaldo, B.J., Roller, D., Axelrod, M.J., Koeppel, A.F., Petricoin, E.F., Slingluff, C.L., Jr., Weber, M.J., Mackey, A.J., Gioeli, D., and Bekiranov, S. (2015). Systems Analysis of Adaptive Responses to MAP Kinase Pathway Blockade in BRAF Mutant Melanoma. PLoS ONE 10, e0138210.

Cappuccio, A., Zollinger, R., Schenk, M., Walczak, A., Servant, N., Barillot, E., Hupé, P., Modlin, R.L., and Soumelis, V. (2015). Combinatorial code governing cellular responses to complex stimuli. Nat. Commun. 6, 6847.

Carnero, A., Blanco-Aparicio, C., Renner, O., Link, W., and Leal, J.F.M. (2008). The PTEN/PI3K/AKT signalling pathway in cancer, therapeutic implications. Curr. Cancer Drug Targets 8, 187-198.

Chandarlapaty, S., Sawai, A., Scaltriti, M., Rodrik-Outmezguine, V., Grbovic-Huezo, O., Serra, V., Majumder, P.K., Baselga, J., and Rosen, N. (2011). AKT inhibition relieves feedback suppression of receptor tyrosine kinase expression and activity. Cancer Cell 19, 58-71.

Chapman, P.B., Hauschild, A., Robert, C., Haanen, J.B., Ascierto, P., Larkin, J., Dummer, R., Garbe, C., Testori, A., Maio, M., et al. (2011). Improved survival with vemurafenib in melanoma with BRAF V600E mutation. N. Engl. J. Med. 364, 2507-2516.

Chapman, P.B., Solit, D.B., and Rosen, N. (2014). Combination of RAF and MEK inhibition for the treatment of BRAF-mutated melanoma: feedback is not encouraged. Cancer Cell 26, 603-604.

Ci, X., Li, B., Ma, X., Kong, F., Zheng, C., Björkholm, M., Jia, J., and Xu, D. (2015). Bortezomib-mediated down-regulation of telomerase and disruption of telomere homeostasis contributes to apoptosis of malignant cells. Oncotarget 6, 3807938092 .

Conway, K., Edmiston, S.N., Khondker, Z.S., Groben, P.A., Zhou, X., Chu, H., Kuan, P.F., Hao, H., Carson, C., Berwick, M., et al. (2011). DNA-methylation profiling distinguishes malignant melanomas from benign nevi. Pigment Cell Melanoma Res. $24,352-360$.

Corcoran, R.B., Dias-Santagata, D., Bergethon, K., Iafrate, A.J., Settleman, J., and Engelman, J.A. (2010). BRAF Gene Amplification Can Promote Acquired Resistance 
to MEK Inhibitors in Cancer Cells Harboring the BRAF V600E Mutation. Sci. Signal. 3, ra84.

Corcoran, R.B., Rothenberg, S.M., Hata, A.N., Faber, A.C., Piris, A., Nazarian, R.M., Brown, R.D., Godfrey, J.T., Winokur, D., Walsh, J., et al. (2013). TORC1 Suppression Predicts Responsiveness to RAF and MEK Inhibition in BRAF-Mutant Melanoma. Sci. Transl. Med. 5, 196ra98-196ra98.

Curtin, J.A., Fridlyand, J., Kageshita, T., Patel, H.N., Busam, K.J., Kutzner, H., Cho, K.-H., Aiba, S., Bröcker, E.-B., LeBoit, P.E., et al. (2005). Distinct sets of genetic alterations in melanoma. N. Engl. J. Med. 353, 2135-2147.

Dalporto, J. (2004). B cell antigen receptor signaling 101. Mol. Immunol. 41, 599613.

Dastidar, S.G., Ganguly, K., Chaudhuri, K., and Chakrabarty, A.N. (2000). The antibacterial action of diclofenac shown by inhibition of DNA synthesis. Int. J. Antimicrob. Agents 14, 249-251.

Davies, M.A. (2012). The role of the PI3K-AKT pathway in melanoma. Cancer J. Sudbury Mass 18, 142-147.

Davies, H., Bignell, G.R., Cox, C., Stephens, P., Edkins, S., Clegg, S., Teague, J., Woffendin, H., Garnett, M.J., Bottomley, W., et al. (2002). Mutations of the BRAF gene in human cancer. Nature 417, 949-954.

Deglesne, P.-A. (2006). Survival Response to B-Cell Receptor Ligation Is Restricted to Progressive Chronic Lymphocytic Leukemia Cells Irrespective of Zap70 Expression. Cancer Res. 66, 7158-7166.

Delcourt, N., Bockaert, J., and Marin, P. (2007). GPCR-jacking: from a new route in RTK signalling to a new concept in GPCR activation. Trends Pharmacol. Sci. 28, 602607.

Delmas, A., Cherier, J., Pohorecka, M., Medale-Giamarchi, C., Meyer, N., Casanova, A., Sordet, O., Lamant, L., Savina, A., Pradines, A., et al. (2015). The c-Jun/RHOB/AKT pathway confers resistance of BRAF -mutant melanoma cells to MAPK inhibitors. Oncotarget 6, 15250-15264.

Denecker, G., Vandamme, N., Akay, O., Koludrovic, D., Taminau, J., Lemeire, K., Gheldof, A., De Craene, B., Van Gele, M., Brochez, L., et al. (2014). Identification of a ZEB2-MITF-ZEB1 transcriptional network that controls melanogenesis and melanoma progression. Cell Death Differ. 21, 1250-1261.

Deuker, M.M., Marsh Durban, V., Phillips, W.A., and McMahon, M. (2015). PI3'-kinase inhibition forestalls the onset of MEK1/2 inhibitor resistance in BRAF-mutated melanoma. Cancer Discov. 5, 143-153. 
Dibb, N.J., Dilworth, S.M., and Mol, C.D. (2004). Opinion: Switching on kinases: oncogenic activation of BRAF and the PDGFR family. Nat. Rev. Cancer 4, 718-727.

Diella, F., Gould, C.M., Chica, C., Via, A., and Gibson, T.J. (2008). Phospho.ELM: a database of phosphorylation sites-update 2008. Nucleic Acids Res. 36, D240-D244.

Dolmetsch, R.E., Lewis, R.S., Goodnow, C.C., and Healy, J.I. (1997). Differential activation of transcription factors induced by $\mathrm{Ca} 2+$ response amplitude and duration. Nature $386,855-858$.

Dong, C., Davis, R.J., and Flavell, R.A. (2002). MAP kinases in the immune response. Annu. Rev. Immunol. 20, 55-72.

Downward, J. (2003). Targeting RAS signalling pathways in cancer therapy. Nat. Rev. Cancer 3, 11-22.

Draghici, S., Khatri, P., Martins, R.P., Ostermeier, G.C., and Krawetz, S.A. (2003). Global functional profiling of gene expression. Genomics 81, 98-104.

Draghici, S., Khatri, P., Tarca, A.L., Amin, K., Done, A., Voichita, C., Georgescu, C., and Romero, R. (2007). A systems biology approach for pathway level analysis. Genome Res. 17, 000-000.

Du, P., Kibbe, W.A., and Lin, S.M. (2008). lumi: a pipeline for processing Illumina microarray. Bioinformatics 24, 1547-1548.

Dudoit, S., and Fridlyand, J. (2002). A prediction-based resampling method for estimating the number of clusters in a dataset. Genome Biol. 3, RESEARCH0036.

Efroni, S., Schaefer, C.F., and Buetow, K.H. (2007). Identification of key processes underlying cancer phenotypes using biologic pathway analysis. PloS One 2, e425.

Eichhoff, O.M., Weeraratna, A., Zipser, M.C., Denat, L., Widmer, D.S., Xu, M., Kriegl, L., Kirchner, T., Larue, L., Dummer, R., et al. (2011). Differential LEF1 and TCF4 expression is involved in melanoma cell phenotype switching. Pigment Cell Melanoma Res. 24, 631-642.

Ernfors, P. (2010). Cellular origin and developmental mechanisms during the formation of skin melanocytes. Exp. Cell Res. 316, 1397-1407.

Fabian, J.R., Daar, I.O., and Morrison, D.K. (1993). Critical tyrosine residues regulate the enzymatic and biological activity of Raf-1 kinase. Mol. Cell. Biol. 13, 7170-7179.

Falchook, G.S. (2012). Activity of the oral MEK inhibitor trametinib in patients with advanced melanoma: a phase 1 dose-escalation trial. Lancet Oncol 13, 782-789.

Farrar, M.A., Alberol-Ila, J., and Perlmutter, R.M. (1996). Activation of the Raf-1 kinase cascade by coumermycin-induced dimerization. Nature 383, 178-181. 
Fattore, L., Marra, E., Pisanu, M.E., Noto, A., de Vitis, C., Belleudi, F., Aurisicchio, L., Mancini, R., Torrisi, M.R., Ascierto, P.A., et al. (2013). Activation of an early feedback survival loop involving phospho-ErbB3 is a general response of melanoma cells to RAF/MEK inhibition and is abrogated by anti-ErbB3 antibodies. J. Transl. Med. 11, 180.

Fattore, L., Malpicci, D., Marra, E., Belleudi, F., Noto, A., De Vitis, C., Pisanu, M.E., Coluccia, P., Camerlingo, R., Roscilli, G., et al. (2015). Combination of antibodies directed against different ErbB3 surface epitopes prevents the establishment of resistance to BRAF/MEK inhibitors in melanoma. Oncotarget 6, 24823-24841.

Federici, G., Gao, X., Slawek, J., Arodz, T., Shitaye, A., Wulfkuhle, J.D., Maria, R.D., Liotta, L.A., and Petricoin, E.F. (2013). Systems Analysis of the NCI-60 Cancer Cell Lines by Alignment of Protein Pathway Activation Modules with "-OMIC" Data Fields and Therapeutic Response Signatures. Mol. Cancer Res. 11, 676-685.

Flaherty, K.T., Puzanov, I., Kim, K.B., Ribas, A., McArthur, G.A., Sosman, J.A., O’Dwyer, P.J., Lee, R.J., Grippo, J.F., Nolop, K., et al. (2010). Inhibition of Mutated, Activated BRAF in Metastatic Melanoma. N. Engl. J. Med. 363, 809-819.

Flaherty, K.T., Robert, C., Hersey, P., Nathan, P., Garbe, C., Milhem, M., Demidov, L.V., Hassel, J.C., Rutkowski, P., Mohr, P., et al. (2012a). Improved Survival with MEK Inhibition in BRAF-Mutated Melanoma. N. Engl. J. Med. 367, 107-114.

Flaherty, K.T., Infante, J.R., Daud, A., Gonzalez, R., Kefford, R.F., Sosman, J., Hamid, O., Schuchter, L., Cebon, J., Ibrahim, N., et al. (2012b). Combined BRAF and MEK Inhibition in Melanoma with BRAF V600 Mutations. N. Engl. J. Med. 367, 1694-1703.

Flaswinkel, H., and Reth, M. (1994). Dual role of the tyrosine activation motif of the Ig-alpha protein during signal transduction via the B cell antigen receptor. EMBO J. 13, 83-89.

Garapaty-Rao, S., Nasveschuk, C., Gagnon, A., Chan, E.Y., Sandy, P., Busby, J., Balasubramanian, S., Campbell, R., Zhao, F., Bergeron, L., et al. (2013). Identification of EZH2 and EZH1 small molecule inhibitors with selective impact on diffuse large B cell lymphoma cell growth. Chem. Biol. 20, 1329-1339.

Garraway, L.A., and Jänne, P.A. (2012). Circumventing Cancer Drug Resistance in the Era of Personalized Medicine. Cancer Discov. 2, 214-226.

Garraway, L.A., Widlund, H.R., Rubin, M.A., Getz, G., Berger, A.J., Ramaswamy, S., Beroukhim, R., Milner, D.A., Granter, S.R., Du, J., et al. (2005). Integrative genomic analyses identify MITF as a lineage survival oncogene amplified in malignant melanoma. Nature 436, 117-122.

Gazzoli, I., Loda, M., Garber, J., Syngal, S., and Kolodner, R.D. (2002). A hereditary nonpolyposis colorectal carcinoma case associated with hypermethylation of the 
MLH1 gene in normal tissue and loss of heterozygosity of the unmethylated allele in the resulting microsatellite instability-high tumor. Cancer Res. 62, 3925-3928.

Gentleman, R.C., Carey, V.J., Bates, D.M., Bolstad, B., Dettling, M., Dudoit, S., Ellis, B., Gautier, L., Ge, Y., Gentry, J., et al. (2004). Bioconductor: open software development for computational biology and bioinformatics. Genome Biol. 5, R80.

Gioeli, D., Wunderlich, W., Sebolt-Leopold, J., Bekiranov, S., Wulfkuhle, J.D., Petricoin, E.F., Conaway, M., and Weber, M.J. (2011). Compensatory pathways induced by MEK inhibition are effective drug targets for combination therapy against castrationresistant prostate cancer. Mol. Cancer Ther. 10, 1581-1590.

Goel, V.K., Lazar, A.J.F., Warneke, C.L., Redston, M.S., and Haluska, F.G. (2006).

Examination of mutations in BRAF, NRAS, and PTEN in primary cutaneous melanoma. J. Invest. Dermatol. 126, 154-160.

Gold, M.R., Ingham, R.J., McLeod, S.J., Christian, S.L., Scheid, M.P., Duronio, V., Santos, L., and Matsuuchi, L. (2000). Targets of B-cell antigen receptor signaling: the phosphatidylinositol 3-kinase/Akt/glycogen synthase kinase-3 signaling pathway and the Rap1 GTPase. Immunol. Rev. 176, 47-68.

Goldstein, A.M. (2004). Familial melanoma, pancreatic cancer and germline CDKN2A mutations. Hum. Mutat. 23, 630-630.

Golub, T.R., Slonim, D.K., Tamayo, P., Huard, C., Gaasenbeek, M., Mesirov, J.P., Coller, H., Loh, M.L., Downing, J.R., Caligiuri, M.A., et al. (1999). Molecular classification of cancer: class discovery and class prediction by gene expression monitoring. Science 286, 531-537.

Gopal, Y.N.V., Rizos, H., Chen, G., Deng, W., Frederick, D.T., Cooper, Z.A., Scolyer, R.A., Pupo, G., Komurov, K., Sehgal, V., et al. (2014). Inhibition of mTORC1/2 overcomes resistance to MAPK pathway inhibitors mediated by PGC1 $\alpha$ and oxidative phosphorylation in melanoma. Cancer Res. 74, 7037-7047.

Gray-Schopfer, V., Wellbrock, C., and Marais, R. (2007). Melanoma biology and new targeted therapy. Nature 445, 851-857.

Greaves, W.O., Verma, S., Patel, K.P., Davies, M.A., Barkoh, B.A., Galbincea, J.M., Yao, H., Lazar, A.J., Aldape, K.D., Medeiros, L.J., et al. (2013). Frequency and spectrum of BRAF mutations in a retrospective, single-institution study of 1112 cases of melanoma. J. Mol. Diagn. JMD 15, 220-226.

Halaban, R., Krauthammer, M., Pelizzola, M., Cheng, E., Kovacs, D., Sznol, M., Ariyan, S., Narayan, D., Bacchiocchi, A., Molinaro, A., et al. (2009). Integrative Analysis of Epigenetic Modulation in Melanoma Cell Response to Decitabine: Clinical Implications. PLoS ONE 4. 
Halaban, R., Zhang, W., Bacchiocchi, A., Cheng, E., Parisi, F., Ariyan, S., Krauthammer, M., McCusker, J.P., Kluger, Y., and Sznol, M. (2010). PLX4032, a selective BRAFV600E kinase inhibitor, activates the ERK pathway and enhances cell migration and proliferation of BRAFWT melanoma cells. Pigment Cell Melanoma Res. 23, 190-200.

Hanafusa, H., Torii, S., Yasunaga, T., and Nishida, E. (2002). Sprouty1 and Sprouty2 provide a control mechanism for the Ras/MAPK signalling pathway. Nat Cell Biol 4, 850-858.

Hanahan, D., and Weinberg, R.A. (2000). The hallmarks of cancer. Cell 100, 57-70.

Hanahan, D., and Weinberg, R.A. (2011). Hallmarks of Cancer: The Next Generation. Cell 144, 646-674.

Hartsough, E.J., and Aplin, A.E. (2013). A STATement on Vemurafenib-Resistant Melanoma. J. Invest. Dermatol. 133, 1928-1929.

Hartsough, E., Shao, Y., and Aplin, A.E. (2014). Resistance to RAF inhibitors revisited. J. Invest. Dermatol. 134, 319-325.

Hauschild, A., Grob, J.-J., Demidov, L.V., Jouary, T., Gutzmer, R., Millward, M., Rutkowski, P., Blank, C.U., Miller, W.H., Kaempgen, E., et al. (2012). Dabrafenib in BRAF-mutated metastatic melanoma: a multicentre, open-label, phase 3 randomised controlled trial. Lancet 380, 358-365.

Held, M.A., Langdon, C.G., Platt, J.T., Graham-Steed, T., Liu, Z., Chakraborty, A., Bacchiocchi, A., Koo, A., Haskins, J.W., Bosenberg, M.W., et al. (2013). Genotypeselective combination therapies for melanoma identified by high-throughput drug screening. Cancer Discov. 3, 52-67.

Herman, S.E.M., Gordon, A.L., Hertlein, E., Ramanunni, A., Zhang, X., Jaglowski, S., Flynn, J., Jones, J., Blum, K.A., Buggy, J.J., et al. (2011). Bruton tyrosine kinase represents a promising therapeutic target for treatment of chronic lymphocytic leukemia and is effectively targeted by PCI-32765. Blood 117, 6287-6296.

Hertzman Johansson, C., Azimi, A., Frostvik Stolt, M., Shojaee, S., Wiberg, H., Grafström, E., Hansson, J., and Egyházi Brage, S. (2013). Association of MITF and other melanosome-related proteins with chemoresistance in melanoma tumors and cell lines. Melanoma Res.

Hodis, E., Watson, I.R., Kryukov, G.V., Arold, S.T., Imielinski, M., Theurillat, J.-P., Nickerson, E., Auclair, D., Li, L., Place, C., et al. (2012). A landscape of driver mutations in melanoma. Cell 150, 251-263.

Hoeflich, K.P., Merchant, M., Orr, C., Chan, J., Den Otter, D., Berry, L., Kasman, I., Koeppen, H., Rice, K., Yang, N.-Y., et al. (2012). Intermittent administration of MEK 
inhibitor GDC-0973 plus PI3K inhibitor GDC-0941 triggers robust apoptosis and tumor growth inhibition. Cancer Res. 72, 210-219.

Holderfield, M., Nagel, T.E., and Stuart, D.D. (2014). Mechanism and consequences of RAF kinase activation by small-molecule inhibitors. Br. J. Cancer 111, 640-645.

Honigberg, L.A., Smith, A.M., Sirisawad, M., Verner, E., Loury, D., Chang, B., Li, S., Pan, Z., Thamm, D.H., Miller, R.A., et al. (2010). The Bruton tyrosine kinase inhibitor PCI32765 blocks B-cell activation and is efficacious in models of autoimmune disease and B-cell malignancy. Proc. Natl. Acad. Sci. 107, 13075-13080.

Improta, G., Zupa, A., Fillmore, H., Deng, J., Aieta, M., Musto, P., Liotta, L.A., Broaddus, W., Petricoin, E.F., and Wulfkuhle, J.D. (2011). Protein Pathway Activation Mapping of Brain Metastasis from Lung and Breast Cancers Reveals Organ Type Specific Drug Target Activation. J. Proteome Res. 10, 3089-3097.

Ji, Z., Erin Chen, Y., Kumar, R., Taylor, M., Jenny Njauw, C.-N., Miao, B., Frederick, D.T., Wargo, J.A., Flaherty, K.T., Jönsson, G., et al. (2015). MITF Modulates Therapeutic Resistance through EGFR Signaling. J. Invest. Dermatol.

Johannessen, C.M., Boehm, J.S., Kim, S.Y., Thomas, S.R., Wardwell, L., Johnson, L.A., Emery, C.M., Stransky, N., Cogdill, A.P., Barretina, J., et al. (2010). COT drives resistance to RAF inhibition through MAP kinase pathway reactivation. Nature 468, 968-972.

Johannessen, C.M., Johnson, L.A., Piccioni, F., Townes, A., Frederick, D.T., Donahue, M.K., Narayan, R., Flaherty, K.T., Wargo, J.A., Root, D.E., et al. (2013). A melanocyte lineage program confers resistance to MAP kinase pathway inhibition. Nature 504, 138-142.

Johnson, G.L., and Lapadat, R. (2002). Mitogen-activated protein kinase pathways mediated by ERK, JNK, and p38 protein kinases. Science 298, 1911-1912.

Joseph, E.W., Pratilas, C.A., Poulikakos, P.I., Tadi, M., Wang, W., Taylor, B.S., Halilovic, E., Persaud, Y., Xing, F., Viale, A., et al. (2010). The RAF inhibitor PLX4032 inhibits ERK signaling and tumor cell proliferation in a V600E BRAF-selective manner. Proc. Natl. Acad. Sci. 107, 14903-14908.

Joshi-Tope, G., Gillespie, M., Vastrik, I., D’Eustachio, P., Schmidt, E., de Bono, B., Jassal, B., Gopinath, G.R., Wu, G.R., Matthews, L., et al. (2005). Reactome: a knowledgebase of biological pathways. Nucleic Acids Res. 33, D428-432.

Keating, G.M., and Santoro, A. (2009). Sorafenib. Drugs 69, 223-240.

Keizer, P.L.J. de, Packer, L.M., Szypowska, A.A., Riedl-Polderman, P.E., Broek, N.J.F. van den, Bruin, A. de, Dansen, T.B., Marais, R., Brenkman, A.B., and Burgering, B.M.T. 
(2010). Activation of Forkhead Box 0 Transcription Factors by Oncogenic BRAF Promotes p21cip1-Dependent Senescence. Cancer Res. 70, 8526-8536.

Kerr, M.K., Martin, M., and Churchill, G.A. (2000). Analysis of variance for gene expression microarray data. J. Comput. Biol. J. Comput. Mol. Cell Biol. 7, 819-837.

Khatri, P., Draghici, S., Ostermeier, G.C., and Krawetz, S.A. (2002). Profiling gene expression using onto-express. Genomics 79, 266-270.

Kitamura, D., Roes, J., Kühn, R., and Rajewsky, K. (1991). A B cell-deficient mouse by targeted disruption of the membrane exon of the immunoglobulin mu chain gene. Nature 350, 423-426.

Kitamura, D., Kudo, A., Schaal, S., Müller, W., Melchers, F., and Rajewsky, K. (1992). A critical role of lambda 5 protein in B cell development. Cell 69, 823-831.

Konieczkowski, D.J., Johannessen, C.M., Abudayyeh, O., Kim, J.W., Cooper, Z.A., Piris, A., Frederick, D.T., Barzily-Rokni, M., Straussman, R., Haq, R., et al. (2014). A Melanoma Cell State Distinction Influences Sensitivity to MAPK Pathway Inhibitors. Cancer Discov. 4, 816-827.

Kugel, C.H., Hartsough, E.J., Davies, M.A., Setiady, Y.Y., and Aplin, A.E. (2014). Function-Blocking ERBB3 Antibody Inhibits the Adaptive Response to RAF Inhibitor. Cancer Res.

Kurosaki, T. (1999). Genetic analysis of B cell antigen receptor signaling. Annu. Rev. Immunol. 17, 555-592.

Langdon, C.G., Held, M.A., Platt, J.T., Meeth, K., Iyidogan, P., Mamillapalli, R., Koo, A.B., Klein, M., Liu, Z., Bosenberg, M.W., et al. (2015). The broad-spectrum receptor tyrosine kinase inhibitor dovitinib suppresses growth of BRAF-mutant melanoma cells in combination with other signaling pathway inhibitors. Pigment Cell Melanoma Res.

Lassen, A., Atefi, M., Robert, L., Wong, D.J., Cerniglia, M., Comin-Anduix, B., and Ribas, A. (2014). Effects of AKT inhibitor therapy in response and resistance to BRAF inhibition in melanoma. Mol. Cancer 13, 83.

Lawrence, M.S., Stojanov, P., Mermel, C.H., Robinson, J.T., Garraway, L.A., Golub, T.R., Meyerson, M., Gabriel, S.B., Lander, E.S., and Getz, G. (2014). Discovery and saturation analysis of cancer genes across 21 tumour types. Nature 505, 495-501.

Lee, F.S., and Chao, M.V. (2001). Activation of Trk neurotrophin receptors in the absence of neurotrophins. Proc. Natl. Acad. Sci. 98, 3555-3560. 
Lee, F.S., Rajagopal, R., Kim, A.H., Chang, P.C., and Chao, M.V. (2002). Activation of Trk Neurotrophin Receptor Signaling by Pituitary Adenylate Cyclase-activating Polypeptides. J. Biol. Chem. 277, 9096-9102.

Levin-Gromiko, U., Koshelev, V., Kushnir, P., Fedida-Metula, S., Voronov, E., and Fishman, D. (2014). Amplified lipid rafts of malignant cells constitute a target for inhibition of aberrantly active NFAT and melanoma tumor growth by the aminobisphosphonate zoledronic acid. Carcinogenesis 35, 2555-2566.

Li, Q., Li, J., Wen, T., Zeng, W., Peng, C., Yan, S., Tan, J., Yang, K., Liu, S., Guo, A., et al. (2014). Overexpression of HMGB1 in melanoma predicts patient survival and suppression of HMGB1 induces cell cycle arrest and senescence in association with p21 (Waf1/Cip1) up-regulation via a p53-independent, Sp1-dependent pathway. Oncotarget 5, 6387-6403.

Lin, L., Sabnis, A.J., Chan, E., Olivas, V., Cade, L., Pazarentzos, E., Asthana, S., Neel, D., Yan, J.J., Lu, X., et al. (2015). The Hippo effector YAP promotes resistance to RAF- and MEK-targeted cancer therapies. Nat. Genet. 47, 250-256.

Lito, P., Pratilas, C.A., Joseph, E.W., Tadi, M., Halilovic, E., Zubrowski, M., Huang, A., Wong, W.L., Callahan, M.K., Merghoub, T., et al. (2012). Relief of profound feedback inhibition of mitogenic signaling by RAF inhibitors attenuates their activity in BRAFV600E melanomas. Cancer Cell 22, 668-682.

Lito, P., Rosen, N., and Solit, D.B. (2013). Tumor adaptation and resistance to RAF inhibitors. Nat. Med. 19, 1401-1409.

Liu, H., Johnson, J.L., Koval, G., Malnassy, G., Sher, D., Damon, L.E., Hsi, E.D., Bucci, D.M., Linker, C.A., Cheson, B.D., et al. (2012). Detection of minimal residual disease following induction immunochemotherapy predicts progression free survival in mantle cell lymphoma: final results of CALGB 59909. Haematologica 97, 579-585.

Liu, Z.-J., Xiao, M., Balint, K., Smalley, K.S.M., Brafford, P., Qiu, R., Pinnix, C.C., Li, X., and Herlyn, M. (2006). Notch1 Signaling Promotes Primary Melanoma Progression by Activating Mitogen-Activated Protein Kinase/Phosphatidylinositol 3-Kinase-Akt Pathways and Up-regulating N-Cadherin Expression. Cancer Res. 66, 4182-4190.

Luciani, F., Champeval, D., Herbette, A., Denat, L., Aylaj, B., Martinozzi, S., Ballotti, R., Kemler, R., Goding, C.R., De Vuyst, F., et al. (2011). Biological and mathematical modeling of melanocyte development. Dev. Camb. Engl. 138, 3943-3954.

Luo, Z. (1996). Oligomerization activates c-Raf-1 through a Ras-dependent mechanism. Nature 383, 181-185.

Ma, X.-H., Piao, S.-F., Dey, S., Mcafee, Q., Karakousis, G., Villanueva, J., Hart, L.S., Levi, S., Hu, J., Zhang, G., et al. (2014). Targeting ER stress-induced autophagy overcomes BRAF inhibitor resistance in melanoma. J. Clin. Invest. 124, 1406-1417. 
Marais, R., Light, Y., Paterson, H.F., Mason, C.S., and Marshall, C.J. (1997). Differential regulation of Raf-1, A-Raf, and B-Raf by oncogenic ras and tyrosine kinases. J. Biol. Chem. 272, 4378-4383.

Martz, C.A., Ottina, K.A., Singleton, K.R., Jasper, J.S., Wardell, S.E., Peraza-Penton, A., Anderson, G.R., Winter, P.S., Wang, T., Alley, H.M., et al. (2014). Systematic identification of signaling pathways with potential to confer anticancer drug resistance. Sci. Signal. 7, ra121.

Mason, C.S., Springer, C.J., Cooper, R.G., Superti-Furga, G., Marshall, C.J., and Marais, R. (1999). Serine and tyrosine phosphorylations cooperate in Raf-1, but not B-Raf activation. EMBO J. 18, 2137-2148.

Matallanas, D., Birtwistle, M., Romano, D., Zebisch, A., Rauch, J., von Kriegsheim, A., and Kolch, W. (2011). Raf family kinases: old dogs have learned new tricks. Genes Cancer 2, 232-260.

McCubrey, J.A., Steelman, L.S., Abrams, S.L., Lee, J.T., Chang, F., Bertrand, F.E., Navolanic, P.M., Terrian, D.M., Franklin, R.A., D’Assoro, A.B., et al. (2006). Roles of the RAF/MEK/ERK and PI3K/PTEN/AKT pathways in malignant transformation and drug resistance. Adv. Enzyme Regul. 46, 249-279.

Michaelis, M., Rothweiler, F., Nerreter, T., Van Rikxoort, M., Sharifi, M., Wiese, M., Ghafourian, T., and Cinatl, J. (2014). Differential effects of the oncogenic BRAF inhibitor PLX4032 (vemurafenib) and its progenitor PLX4720 on ABCB1 function. J. Pharm. Pharm. Sci. Publ. Can. Soc. Pharm. Sci. Société Can. Sci. Pharm. 17, 154-168.

Molhoek, K.R., Shada, A.L., Smolkin, M., Chowbina, S., Papin, J., Brautigan, D.L., and Slingluff, C.L. (2011). Comprehensive analysis of RTK activation in human melanomas reveals autocrine signaling through IGF-1R. Melanoma Res. 21, 274284.

Montagut, C. (2008). Elevated CRAF as a potential mechanism of acquired resistance to BRAF inhibition in melanoma. Cancer Res 68, 4853-4861.

Müller, J., Krijgsman, O., Tsoi, J., Robert, L., Hugo, W., Song, C., Kong, X., Possik, P.A., Cornelissen-Steijger, P.D.M., Foppen, M.H.G., et al. (2014). Low MITF/AXL ratio predicts early resistance to multiple targeted drugs in melanoma. Nat. Commun. 5.

Nazarian, R., Shi, H., Wang, Q., Kong, X., Koya, R.C., Lee, H., Chen, Z., Lee, M.-K., Attar, N., Sazegar, H., et al. (2010). Melanomas acquire resistance to B-RAF(V600E) inhibition by RTK or N-RAS upregulation. Nature 468, 973-977.

Niezgoda, A., Niezgoda, P., and Czajkowski, R. (2015). Novel Approaches to Treatment of Advanced Melanoma: A Review on Targeted Therapy and Immunotherapy. BioMed Res. Int. 2015, 851387. 
Ogata, H., Goto, S., Sato, K., Fujibuchi, W., Bono, H., and Kanehisa, M. (1999). KEGG: Kyoto Encyclopedia of Genes and Genomes. Nucleic Acids Res. 27, 29-34.

Oikawa, T., and Yamada, T. (2003). Molecular biology of the Ets family of transcription factors. Gene 303, 11-34.

Oike, T., Ogiwara, H., Nakano, T., Yokota, J., and Kohno, T. (2013). Inactivating mutations in SWI/SNF chromatin remodeling genes in human cancer. Jpn. J. Clin. Oncol. 43, 849-855.

Osawa (2009). Melanocyte stem cells. StemBook.

Pantziarka, P., Sukhatme, V., Bouche, G., Meheus, L., and Sukhatme, V.P. (2016). Repurposing Drugs in Oncology (ReDO)—diclofenac as an anti-cancer agent. Ecancermedicalscience 10.

Pao, L.I., Famiglietti, S.J., and Cambier, J.C. (1998). Asymmetrical phosphorylation and function of immunoreceptor tyrosine-based activation motif tyrosines in B cell antigen receptor signal transduction. J. Immunol. Baltim. Md 1950 160, 3305-3314.

Paraiso, K.H.T., Fedorenko, I.V., Cantini, L.P., Munko, A.C., Hall, M., Sondak, V.K., Messina, J.L., Flaherty, K.T., and Smalley, K.S.M. (2010). Recovery of phospho-ERK activity allows melanoma cells to escape from BRAF inhibitor therapy. Br. J. Cancer $102,1724-1730$.

Parsons, D.W., Jones, S., Zhang, X., Lin, J.C.-H., Leary, R.J., Angenendt, P., Mankoo, P., Carter, H., Siu, I.-M., Gallia, G.L., et al. (2008). An integrated genomic analysis of human glioblastoma multiforme. Science 321, 1807-1812.

Pelletier, J., Graff, J., Ruggero, D., and Sonenberg, N. (2015). Targeting the eIF4F translation initiation complex: a critical nexus for cancer development. Cancer Res. $75,250-263$.

Pérez-Galán, P., Dreyling, M., and Wiestner, A. (2011). Mantle cell lymphoma: biology, pathogenesis, and the molecular basis of treatment in the genomic era. Blood 117, 26-38.

Pinheiro, H., Bordeira-Carriço, R., Seixas, S., Carvalho, J., Senz, J., Oliveira, P., Inácio, P., Gusmão, L., Rocha, J., Huntsman, D., et al. (2010). Allele-specific CDH1 downregulation and hereditary diffuse gastric cancer. Hum. Mol. Genet. 19, 943952.

Ponader, S., Chen, S.-S., Buggy, J.J., Balakrishnan, K., Gandhi, V., Wierda, W.G., Keating, M.J., O'Brien, S., Chiorazzi, N., and Burger, J.A. (2012). The Bruton tyrosine kinase inhibitor PCI-32765 thwarts chronic lymphocytic leukemia cell survival and tissue homing in vitro and in vivo. Blood 119, 1182-1189. 
Pott, C., Hoster, E., Delfau-Larue, M.-H., Beldjord, K., Böttcher, S., Asnafi, V., Plonquet, A., Siebert, R., Callet-Bauchu, E., Andersen, N., et al. (2010). Molecular remission is an independent predictor of clinical outcome in patients with mantle cell lymphoma after combined immunochemotherapy: a European MCL intergroup study. Blood $115,3215-3223$.

Poulikakos, P.I., Zhang, C., Bollag, G., Shokat, K.M., and Rosen, N. (2010). RAF inhibitors transactivate RAF dimers and ERK signalling in cells with wild-type BRAF. Nature 464, 427-430.

Poulikakos, P.I., Persaud, Y., Janakiraman, M., Kong, X., Ng, C., Moriceau, G., Shi, H., Atefi, M., Titz, B., Gabay, M.T., et al. (2011). RAF inhibitor resistance is mediated by dimerization of aberrantly spliced BRAF(V600E). Nature 480, 387-390.

Prahallad, A., Sun, C., Huang, S., Di Nicolantonio, F., Salazar, R., Zecchin, D., Beijersbergen, R.L., Bardelli, A., and Bernards, R. (2012). Unresponsiveness of colon cancer to BRAF(V600E) inhibition through feedback activation of EGFR. Nature 483, 100-103.

Pshenichnaya, I., Schouwey, K., Armaro, M., Larue, L., Knoepfler, P.S., Eisenman, R.N., Trumpp, A., Delmas, V., and Beermann, F. (2012). Constitutive gray hair in mice induced by melanocyte-specific deletion of c-Myc. Pigment Cell Melanoma Res. 25, 312-325.

Puente, X.S., Pinyol, M., Quesada, V., Conde, L., Ordóñez, G.R., Villamor, N., Escaramis, G., Jares, P., Beà, S., González-Díaz, M., et al. (2011). Whole-genome sequencing identifies recurrent mutations in chronic lymphocytic leukaemia. Nature 475, 101105.

Rajakulendran, T., Sahmi, M., Lefrancois, M., Sicheri, F., and Therrien, M. (2009). A dimerization-dependent mechanism drives RAF catalytic activation. Nature 461, 542-545.

Rebay, I., Fleming, R.J., Fehon, R.G., Cherbas, L., Cherbas, P., and Artavanis-Tsakonas, S. (1991). Specific EGF repeats of Notch mediate interactions with Delta and Serrate: implications for Notch as a multifunctional receptor. Cell 67, 687-699.

Rebecca, V.W., and Smalley, K.S.M. (2014). Change or die: Targeting adaptive signaling to kinase inhibition in cancer cells. Biochem. Pharmacol. 91, 417-425.

Rebecca, V.W., Massaro, R.R., Fedorenko, I.V., Sondak, V.K., Anderson, A.R.A., Kim, E., Amaravadi, R.K., Maria-Engler, S.S., Messina, J.L., Gibney, G.T., et al. (2014). Inhibition of autophagy enhances the effects of the AKT inhibitor MK-2206 when combined with paclitaxel and carboplatin in BRAF wild-type melanoma. Pigment Cell Melanoma Res. 27, 465-478. 
Roller, D.G., Axelrod, M., Capaldo, B.J., Jensen, K., Mackey, A., Weber, M.J., and Gioeli, D. (2012). Synthetic lethal screening with small-molecule inhibitors provides a pathway to rational combination therapies for melanoma. Mol. Cancer Ther. 11, $2505-2515$.

Roller, D.G., Capaldo, B., Bekiranov, S., Mackey, A.J., Conaway, M.R., Petricoin, E.F., Gioeli, D., and Weber, M.J. (2016). Combinatorial drug screening and molecular profiling reveal diverse mechanisms of intrinsic and adaptive resistance to BRAF inhibition in V600E BRAF mutant melanomas. Oncotarget 7, 2734-2753.

Rowley, R.B., Burkhardt, A.L., Chao, H.G., Matsueda, G.R., and Bolen, J.B. (1995). Syk protein-tyrosine kinase is regulated by tyrosine-phosphorylated Ig alpha/Ig beta immunoreceptor tyrosine activation motif binding and autophosphorylation. J. Biol. Chem. 270, 11590-11594.

Rubinfeld, B., Robbins, P., El-Gamil, M., Albert, I., Porfiri, E., and Polakis, P. (1997). Stabilization of $\beta$-Catenin by Genetic Defects in Melanoma Cell Lines. Science 275, 1790-1792.

Rushworth, L.K., Hindley, A.D., O'Neill, E., and Kolch, W. (2006). Regulation and role of Raf-1/B-Raf heterodimerization. Mol Cell Biol 26, 2262-2272.

Saba, N., and Wiestner, A. (2014). Do mantle cell lymphomas have an "Achilles heel"? Curr. Opin. Hematol. 21, 350-357.

Saijo, K., Mecklenbräuker, I., Santana, A., Leitger, M., Schmedt, C., and Tarakhovsky, A. (2002). Protein kinase $C$ beta controls nuclear factor kappaB activation in $B$ cells through selective regulation of the IkappaB kinase alpha. J. Exp. Med. 195, 16471652.

Saito, K., Scharenberg, A.M., and Kinet, J.P. (2001). Interaction between the Btk PH domain and phosphatidylinositol-3,4,5-trisphosphate directly regulates Btk. J. Biol. Chem. 276, 16201-16206.

Sánchez-Hernández, I., Baquero, P., Calleros, L., and Chiloeches, A. (2011). Dual inhibition of (V600E)BRAF and the PI3K/AKT/mTOR pathway cooperates to induce apoptosis in melanoma cells through a MEK-independent mechanism. Cancer Lett.

Sapkota, B., Hill, C.E., and Pollack, B.P. (2013). Vemurafenib enhances MHC induction in BRAF(V600E) homozygous melanoma cells. Oncoimmunology 2, e22890.

Scharenberg, A.M., El-Hillal, O., Fruman, D.A., Beitz, L.O., Li, Z., Lin, S., Gout, I., Cantley, L.C., Rawlings, D.J., and Kinet, J.P. (1998). Phosphatidylinositol-3,4,5-trisphosphate (PtdIns-3,4,5-P3)/Tec kinase-dependent calcium signaling pathway: a target for SHIP-mediated inhibitory signals. EMBO J. 17, 1961-1972. 
Schneider, P., Schön, M., Pletz, N., Seitz, C.S., Liu, N., Ziegelbauer, K., Zachmann, K., Emmert, S., and Schön, M.P. (2014). The novel PI3 kinase inhibitor, BAY 80-6946, impairs melanoma growth in vivo and in vitro. Exp. Dermatol. 23, 579-584.

Scortegagna, M., Ruller, C., Feng, Y., Lazova, R., Kluger, H., Li, J.-L., De, S.K., Rickert, R., Pellecchia, M., Bosenberg, M., et al. (2014). Genetic inactivation or pharmacological inhibition of Pdk1 delays development and inhibits metastasis of Braf(V600E)::Pten(-/-) melanoma. Oncogene 33, 4330-4339.

Scortegagna, M., Lau, E., Zhang, T., Feng, Y., Sereduk, C., Yin, H., De, S.K., Meeth, K., Platt, J.T., Langdon, C.G., et al. (2015). PDK1 and SGK3 Contribute to the Growth of BRAF-Mutant Melanomas and Are Potential Therapeutic Targets. Cancer Res. 75, 1399-1412.

Shen, X., Liu, Y., Hsu, Y.-J., Fujiwara, Y., Kim, J., Mao, X., Yuan, G.-C., and Orkin, S.H. (2008). EZH1 mediates methylation on histone H3 lysine 27 and complements EZH2 in maintaining stem cell identity and executing pluripotency. Mol. Cell 32, 491-502.

Shi, H., Moriceau, G., Kong, X., Lee, M.-K., Lee, H., Koya, R.C., Ng, C., Chodon, T., Scolyer, R.A., Dahlman, K.B., et al. (2012). Melanoma whole-exome sequencing identifies V600EB-RAF amplification-mediated acquired B-RAF inhibitor resistance. Nat. Commun. 3, 724.

Shi, H., Hong, A., Kong, X., Koya, R.C., Song, C., Moriceau, G., Hugo, W., Yu, C.C., Ng, C., Chodon, T., et al. (2014a). A novel AKT1 mutant amplifies an adaptive melanoma response to BRAF inhibition. Cancer Discov. 4, 69-79.

Shi, H., Hugo, W., Kong, X., Hong, A., Koya, R.C., Moriceau, G., Chodon, T., Guo, R., Johnson, D.B., Dahlman, K.B., et al. (2014b). Acquired resistance and clonal evolution in melanoma during BRAF inhibitor therapy. Cancer Discov. 4, 80-93.

Shimodaira, H. (2002). An Approximately Unbiased Test of Phylogenetic Tree Selection. Syst. Biol. 51, 492-508.

Shtivelman, E., Davies, M.Q.A., Hwu, P., Yang, J., Lotem, M., Oren, M., Flaherty, K.T., and Fisher, D.E. (2014). Pathways and therapeutic targets in melanoma. Oncotarget 5, 1701-1752.

Silva, J.M., Bulman, C., and McMahon, M. (2014). BRAFV600E cooperates with PI3K signaling, independent of AKT, to regulate melanoma cell proliferation. Mol. Cancer Res. MCR 12, 447-463.

Smit, M.A., Maddalo, G., Greig, K., Raaijmakers, L.M., Possik, P.A., van Breukelen, B., Cappadona, S., Heck, A.J., Altelaar, A.M., and Peeper, D.S. (2014). ROCK1 is a potential combinatorial drug target for BRAF mutant melanoma. Mol. Syst. Biol. 10, 772. 
Smyth, G.K. (2004). Linear Models and Empirical Bayes Methods for Assessing Differential Expression in Microarray Experiments. Stat. Appl. Genet. Mol. Biol. 3.

Soda, M., Choi, Y.L., Enomoto, M., Takada, S., Yamashita, Y., Ishikawa, S., Fujiwara, S., Watanabe, H., Kurashina, K., Hatanaka, H., et al. (2007). Identification of the transforming EML4-ALK fusion gene in non-small-cell lung cancer. Nature 448, 561-566.

Solit, D.B., and Rosen, N. (2011). Resistance to BRAF inhibition in melanomas. N Engl J Med 364, 772-774.

Solit, D.B., and Rosen, N. (2014). Towards a unified model of RAF inhibitor resistance. Cancer Discov. 4, 27-30.

Solit, D.B., Garraway, L.A., Pratilas, C.A., Sawai, A., Getz, G., Basso, A., Ye, Q., Lobo, J.M., She, Y., Osman, I., et al. (2006). BRAF mutation predicts sensitivity to MEK inhibition. Nature 439, 358-362.

Søndergaard, J.N., Nazarian, R., Wang, Q., Guo, D., Hsueh, T., Mok, S., Sazegar, H., MacConaill, L.E., Barretina, J.G., Kehoe, S.M., et al. (2010). Differential sensitivity of melanoma cell lines with BRAFV600E mutation to the specific Raf inhibitor PLX4032. J. Transl. Med. 8, 39.

Spagnolo, F., Ghiorzo, P., and Queirolo, P. (2014). Overcoming resistance to BRAF inhibition in BRAF-mutated metastatic melanoma. Oncotarget.

Storey, J.D., and Tibshirani, R. (2003). Statistical methods for identifying differentially expressed genes in DNA microarrays. Methods Mol. Biol. 224, 149157.

Straussman, R., Morikawa, T., Shee, K., Barzily-Rokni, M., Qian, Z.R., Du, J., Davis, A., Mongare, M.M., Gould, J., Frederick, D.T., et al. (2012). Tumour micro-environment elicits innate resistance to RAF inhibitors through HGF secretion. Nature 487, 500504.

Strub, T., Giuliano, S., Ye, T., Bonet, C., Keime, C., Kobi, D., Le Gras, S., Cormont, M., Ballotti, R., Bertolotto, C., et al. (2011). Essential role of microphthalmia transcription factor for DNA replication, mitosis and genomic stability in melanoma. Oncogene 30, 2319-2332.

Subramanian, A., Tamayo, P., Mootha, V.K., Mukherjee, S., Ebert, B.L., Gillette, M.A., Paulovich, A., Pomeroy, S.L., Golub, T.R., Lander, E.S., et al. (2005). Gene set enrichment analysis: A knowledge-based approach for interpreting genome-wide expression profiles. Proc. Natl. Acad. Sci. 102, 15545-15550.

Sullivan, R.J., and Flaherty, K.T. (2013). Resistance to BRAF-targeted therapy in melanoma. Eur. J. Cancer Oxf. Engl. 1990 49, 1297-1304. 
Sumimoto, H., Miyagishi, M., Miyoshi, H., Yamagata, S., Shimizu, A., Taira, K., and Kawakami, Y. (2004). Inhibition of growth and invasive ability of melanoma by inactivation of mutated BRAF with lentivirus-mediated RNA interference. Oncogene 23, 6031-6039.

Suzuki, R., and Shimodaira, H. (2006). Pvclust: an R package for assessing the uncertainty in hierarchical clustering. Bioinformatics 22, 1540-1542.

Swerdlow, S.H., and Williams, M.E. (2002). From centrocytic to mantle cell lymphoma: a clinicopathologic and molecular review of 3 decades. Hum. Pathol. 33, $7-20$.

Teven, C.M., Farina, E.M., Rivas, J., and Reid, R.R. (2014). Fibroblast growth factor (FGF) signaling in development and skeletal diseases. Genes Dis. 1, 199-213.

Thomas, A.J., and Erickson, C.A. (2009). FOXD3 regulates the lineage switch between neural crest-derived glial cells and pigment cells by repressing MITF through a noncanonical mechanism. Dev. Camb. Engl. 136, 1849-1858.

Thomas, N.E., Slater, N.A., Edmiston, S.N., Zhou, X., Kuan, P.-F., Groben, P.A., Carson, C.C., Hao, H., Parrish, E., Moschos, S.J., et al. (2014). DNA-methylation Profiles in Primary Cutaneous Melanomas are Associated with Clinically Significant Pathologic Features. Pigment Cell Melanoma Res. n/a-n/a.

Tiacci, E. (2011). BRAF mutations in hairy-cell leukemia. N Engl J Med 364, 23052315.

Tran, N.H., Wu, X., and Frost, J.A. (2005). B-Raf and Raf-1 are regulated by distinct autoregulatory mechanisms. J. Biol. Chem. 280, 16244-16253.

Trushin, S.A., Pennington, K.N., Algeciras-Schimnich, A., and Paya, C.V. (1999). Protein kinase $C$ and calcineurin synergize to activate IkappaB kinase and NFkappaB in T lymphocytes. J. Biol. Chem. 274, 22923-22931.

Tsai, J., Lee, J.T., Wang, W., Zhang, J., Cho, H., Mamo, S., Bremer, R., Gillette, S., Kong, J., Haass, N.K., et al. (2008). Discovery of a selective inhibitor of oncogenic B-Raf kinase with potent antimelanoma activity. Proc Natl Acad Sci U S A 105, 3041-3046.

Tusher, V.G., Tibshirani, R., and Chu, G. (2001). Significance analysis of microarrays applied to the ionizing radiation response. Proc. Natl. Acad. Sci. U. S. A. 98, 51165121.

Udell, C.M., Rajakulendran, T., Sicheri, F., and Therrien, M. (2011). Mechanistic principles of RAF kinase signaling. Cell. Mol. Life Sci. CMLS 68, 553-565.

Van Allen, E.M., Wagle, N., Sucker, A., Treacy, D.J., Johannessen, C.M., Goetz, E.M., Place, C.S., Taylor-Weiner, A., Whittaker, S., Kryukov, G.V., et al. (2014). The genetic 
landscape of clinical resistance to RAF inhibition in metastatic melanoma. Cancer Discov. 4, 94-109.

Vaske, C.J., Benz, S.C., Sanborn, J.Z., Earl, D., Szeto, C., Zhu, J., Haussler, D., and Stuart, J.M. (2010). Inference of patient-specific pathway activities from multi-dimensional cancer genomics data using PARADIGM. Bioinformatics 26, i237-i245.

Venkatachalam, R., Ligtenberg, M.J.L., Hoogerbrugge, N., Schackert, H.K., Görgens, H., Hahn, M.-M., Kamping, E.J., Vreede, L., Hoenselaar, E., van der Looij, E., et al. (2010). Germline epigenetic silencing of the tumor suppressor gene PTPRJ in early-onset familial colorectal cancer. Gastroenterology 139, 2221-2224.

van de Vijver, M.J., He, Y.D., van't Veer, L.J., Dai, H., Hart, A.A.M., Voskuil, D.W., Schreiber, G.J., Peterse, J.L., Roberts, C., Marton, M.J., et al. (2002). A gene-expression signature as a predictor of survival in breast cancer. N. Engl. J. Med. 347, 1999-2009.

Villanueva, J., Vultur, A., Lee, J.T., Somasundaram, R., Fukunaga-Kalabis, M., Cipolla, A.K., Wubbenhorst, B., Xu, X., Gimotty, P.A., Kee, D., et al. (2010). Acquired resistance to BRAF inhibitors mediated by a RAF kinase switch in melanoma can be overcome by cotargeting MEK and IGF-1R/PI3K. Cancer Cell 18, 683-695.

Wagle, N., Emery, C., Berger, M.F., Davis, M.J., Sawyer, A., Pochanard, P., Kehoe, S.M., Johannessen, C.M., Macconaill, L.E., Hahn, W.C., et al. (2011). Dissecting therapeutic resistance to RAF inhibition in melanoma by tumor genomic profiling. J. Clin. Oncol. Off. J. Am. Soc. Clin. Oncol. 29, 3085-3096.

Wagle, N., Van Allen, E.M., Treacy, D.J., Frederick, D.T., Cooper, Z.A., Taylor-Weiner, A., Rosenberg, M., Goetz, E.M., Sullivan, R.J., Farlow, D.N., et al. (2014). MAP kinase pathway alterations in BRAF-mutant melanoma patients with acquired resistance to combined RAF/MEK inhibition. Cancer Discov. 4, 61-68.

Wang, J., Huang, S.K., Marzese, D.M., Hsu, S.C., Kawas, N.P., Chong, K.K., Long, G.V., Menzies, A.M., Scolyer, R.A., Izraely, S., et al. (2014). Epigenetic Changes of EGFR Play an Important Role in BRAF Inhibitor Resistant Cutaneous Melanomas. J. Invest. Dermatol.

Wang, L., Lawrence, M.S., Wan, Y., Stojanov, P., Sougnez, C., Stevenson, K., Werner, L., Sivachenko, A., DeLuca, D.S., Zhang, L., et al. (2011). SF3B1 and Other Novel Cancer Genes in Chronic Lymphocytic Leukemia. N. Engl. J. Med. 365, 2497-2506.

Wang, M.L., Rule, S., Martin, P., Goy, A., Auer, R., Kahl, B.S., Jurczak, W., Advani, R.H., Romaguera, J.E., Williams, M.E., et al. (2013). Targeting BTK with Ibrutinib in Relapsed or Refractory Mantle-Cell Lymphoma. N. Engl. J. Med. 369, 507-516.

Wellbrock, C., Karasarides, M., and Marais, R. (2004). The RAF proteins take centre stage. Nat. Rev. Mol. Cell Biol. 5, 875-885. 
Wellbrock, C., Rana, S., Paterson, H., Pickersgill, H., Brummelkamp, T., and Marais, R. (2008). Oncogenic BRAF regulates melanoma proliferation through the lineage specific factor MITF. PloS One 3, e2734.

Wilson, T.R., Lee, D.Y., Berry, L., Shames, D.S., and Settleman, J. (2011). Neuregulin-1mediated autocrine signaling underlies sensitivity to HER2 kinase inhibitors in a subset of human cancers. Cancer Cell 20, 158-172.

Wilson, T.R., Fridlyand, J., Yan, Y., Penuel, E., Burton, L., Chan, E., Peng, J., Lin, E., Wang, Y., Sosman, J., et al. (2012). Widespread potential for growth-factor-driven resistance to anticancer kinase inhibitors. Nature 487, 505-509.

Wong, E.M., Southey, M.C., Fox, S.B., Brown, M.A., Dowty, J.G., Jenkins, M.A., Giles, G.G., Hopper, J.L., and Dobrovic, A. (2011). Constitutional methylation of the BRCA1 promoter is specifically associated with BRCA1 mutation-associated pathology in early-onset breast cancer. Cancer Prev. Res. Phila. Pa 4, 23-33.

Woyach, J.A., Furman, R.R., Liu, T.-M., Ozer, H.G., Zapatka, M., Ruppert, A.S., Xue, L., Li, D.H.-H., Steggerda, S.M., Versele, M., et al. (2014). Resistance mechanisms for the Bruton's tyrosine kinase inhibitor ibrutinib. N. Engl. J. Med. 370, 2286-2294.

Xing, F. (2012). Concurrent loss of the PTEN and RB1 tumor suppressors attenuates RAF dependence in melanomas harboring (V600E)BRAF. Oncogene 31, 446-457.

Xu, S., Yang, Z., Zhang, J., Jiang, Y., Chen, Y., Li, H., Liu, X., Xu, D., Chen, Y., Yang, Y., et al. (2014). Increased Levels of $\beta$-catenin, LEF-1, and HPA-1 Correlate with Poor Prognosis for Acral Melanoma with Negative BRAF and NRAS Mutation in BRAF Exons 11 and 15 and NRAS Exons 1 and 2. DNA Cell Biol.

Yadav, V., Zhang, X., Liu, J., Estrem, S., Li, S., Gong, X.-Q., Buchanan, S., Henry, J.R., Starling, J.J., and Peng, S.-B. (2012). Reactivation of mitogen-activated protein kinase (MAPK) pathway by FGF receptor 3 (FGFR3)/Ras mediates resistance to vemurafenib in human B-RAF V600E mutant melanoma. J. Biol. Chem. 287, 2808728098.

Yadav, V., Burke, T.F., Huber, L., Van Horn, R.D., Zhang, Y., Buchanan, S.G., Chan, E.M., Starling, J.J., Beckmann, R.P., and Peng, S.-B. (2014). The CDK4/6 inhibitor LY2835219 overcomes vemurafenib resistance resulting from MAPK reactivation and cyclin D1 upregulation. Mol. Cancer Ther. 13, 2253-2263.

Zhang, B.H., and Guan, K.L. (2000). Activation of B-Raf kinase requires phosphorylation of the conserved residues Thr598 and Ser601. EMBO J. 19, 54295439.

Zhang, K., Wong, P., Duan, J., Jacobs, B., Borden, E.C., and Bedogni, B. (2013). An ERBB3/ERBB2 oncogenic unit plays a key role in NRG1 signaling and melanoma cell growth and survival. Pigment Cell Melanoma Res. 26, 408-414. 
Zhao, W., Sachsenmeier, K., Zhang, L., Sult, E., Hollingsworth, R.E., and Yang, H. (2014). A New Bliss Independence Model to Analyze Drug Combination Data. J. Biomol. Screen. 1087057114521867. 